\title{
ASSESSING THE PHOTOPROTECTIVE EFFECTS OF FLUORESCENT SPHINGOMYELIN AGAINST UVB INDUCED DNA DAMAGE IN HUMAN KERATINOCYTES
}

\author{
A Thesis \\ presented to \\ the Faculty of California Polytechnic State University, \\ San Luis Obispo
}

\author{
In Partial Fulfillment \\ of the Requirements for the Degree \\ Master of Science in Biomedical Engineering
}

by

Rebecca Marie Kandell

June 2018 
(C) 2018

Rebecca Marie Kandell

ALL RIGHTS RESERVED 
TITLE:

AUTHOR:

DATE SUBMITTED:

COMMITTEE CHAIR:

COMMITTEE MEMBER:

COMMITTEE MEMBER:

COMMITTEE MEMBER:
Assessing the Photoprotective Effects of Fluorescent Sphingomyelin Against UVB Induced DNA Damage in Human Keratinocytes

Rebecca Marie Kandell

June 2018

Lily Hsu Laiho, Ph.D.

Professor of Biomedical Engineering

Trevor Cardinal, Ph.D.

Professor of Biomedical Engineering

Kristen O’Halloran Cardinal, Ph.D.

Professor of Biomedical Engineering

Rafael Jimenez-Flores, Ph.D.

Professor of Food, Agricultural, and Environmental Sciences 


\begin{abstract}
Assessing the Photoprotective Effects of Fluorescent Sphingomyelin Against UVB Induced DNA Damage in Human Keratinocytes

Rebecca Marie Kandell
\end{abstract}

Non Melanoma Skin Cancer (NMSC) affects 3.3 million Americans each year and results from Ultra Violet Radiation (UVR) damage to DNA in the form of pyrimidine dimers and photoproducts [1]-[5]. Cells directly detect the damage and initiate apoptosis, cell cycle arrest, or DNA repair by modulating p53 and p21 levels [6]-[9]. Current methods of photoprotection include sunscreen, but controversy over safety of some active ingredients necessitates research into more natural alternatives [10]-[12]. In particular, 24 hour incubation with bovine milk sphingomyelin (BSM) has demonstrated photoprotective potential by reducing p21 and p53 levels in keratinocytes (KRTs) after UV radiation [13], [14]. This thesis aims to expand on past BSM research by exploring the mechanism for photoprotection. Normally, sphingomyelin (SM) is metabolically degraded to ceramide which then leads to cell apoptosis [6]. The goals of this thesis were to characterize a fluorescent SM (FSM) to assess changes in intracellular fluorescence distribution after various incubation and post-UV exposure times. FSM was deemed functionally equivalent to BSM by reducing levels of p21 after UV. Furthermore, quantification demonstrated that FSM trafficking and intracellular fluorescence were independent of continuous incubation time, warranting further investigation into shorter timepoints like 1 hour. Across several post-UV timepoints, the 1 hour incubation had a consistently higher average cytoplasmic mean gray value compared to 24 hour incubation. In addition, the no UV control was significantly lower compared to the 24 hour and 12 hour post-UV timepoints. No post-UV differences were observed for the 24 hour incubation, suggesting future work is necessary for the 1 hour incubation, which potentially streamlines future experiments. Two immunofluorescence stains for endogenous SM (lysenin) and ceramide were also optimized for preliminary fluorescence distribution studies and 
colocalization with FSM. Finally, a 3 T3 fibroblast spheroid model was utilized as proof-ofconcept for future 3D KRT cultures and depth of dye penetration quantification methods. These findings suggest FSM is an appropriate model for BSM trafficking, a shorter FSM incubation time could potentially be adopted in future studies, dual immunofluorescence staining for SM and ceramide is viable, and spheroids provide a promising model for future 3D KRT studies.

Keywords:

Keratinocytes, UV, NMSC, Fluorescent Sphingomyelin, p21, Immunofluorescence, Spheroids 


\section{ACKNOWLEDGMENTS}

I would like to vehemently thank my thesis advisor, Dr. Lily Laiho, for your constant optimism, expertise in confocal imaging, and availability to answer my thesis, career, and personal questions. Thank you for taking a chance on me freshman year! You constantly inspire me to continue my education through a Ph.D. and become a better scientist and person. I would also like to thank Dr. Trevor Cardinal, Dr. Kristen Cardinal, and Dr. Rafael Jimenez-Flores for your willingness to serve on my thesis committee and for your encouragement. In addition, I would like to thank the Hannah Forbes foundation and the Cal Poly Graduate Education program for my Graduate Assistant research fellowship.

My thesis would not be possible without the support of my lab colleagues. I would especially like to thank Jennifer Kandell, for culturing all my cells, helping with image analysis, and always being there when I needed someone to talk to; Tyler Fraser and Stephanie Switalski for developing the incredibly time-saving and useful 2D Fluorescence Distribution ImageJ and MATLAB programs; Leo Banuelos for helping me jumpstart the FSM experiments and for your indispensable image analysis knowledge; Kristina Bishard for training me in lab techniques when I was just starting; Kevin Campbell for passing down the optimized p21 protocol and training me in confocal imaging; and Liz Tseng, Trevor Bingham, and Esther Chen for all the lab laughs.

Finally, I would like to thank my family - mom, dad, grandma, and sister - for your continuous support throughout this entire process. You taught me to persevere and try my best despite any obstacles because "everything comes to those who wait." I am incredibly grateful to have your love and encouragement.

"The cave you fear to enter holds the treasure you seek." - Joseph Campbell 


\section{TABLE OF CONTENTS}

Page

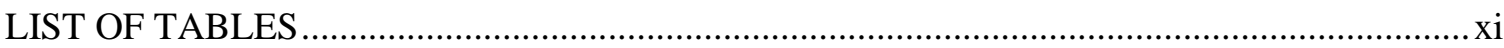

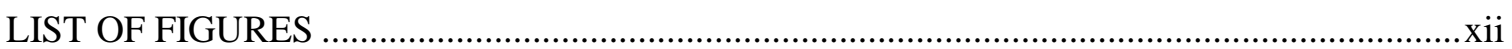

\section{CHAPTER}

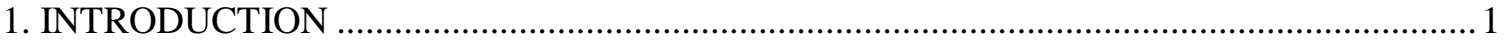

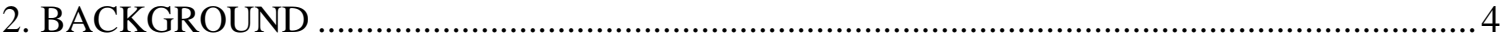

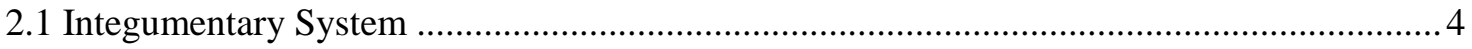

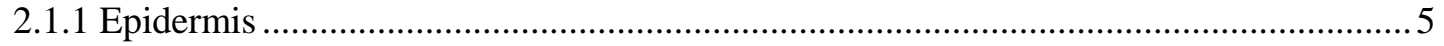

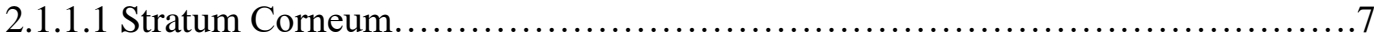

2.1.1.2 Stratum Granulosum and Lucidum........................................... 8

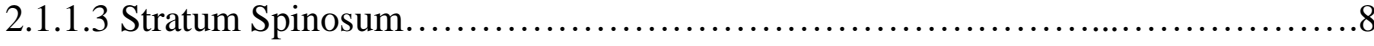

2.1.1.4 Stratum Basale........................................................ 9

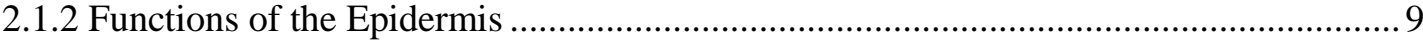

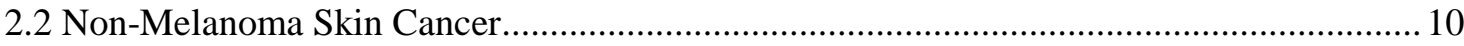

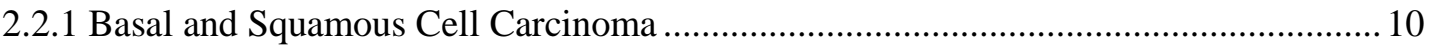

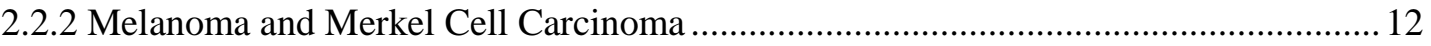

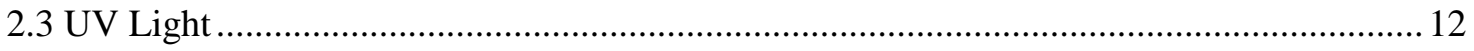

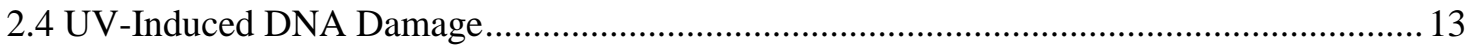

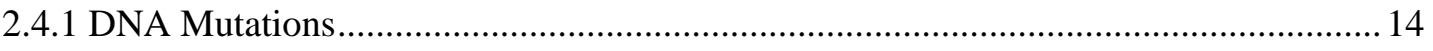

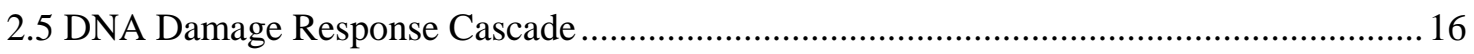

2.5.1 Apoptosis and p53 Tumor Suppressor Gene............................................................ 18

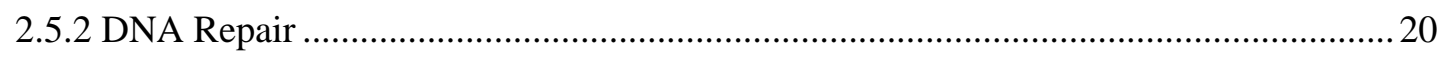

2.5.2.1 Cell Cycle Arrest and P21 Cyclin-CDK Inhibitor ............................20

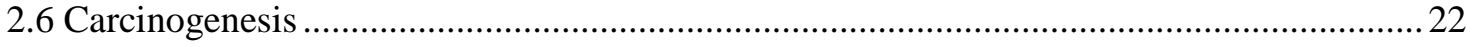

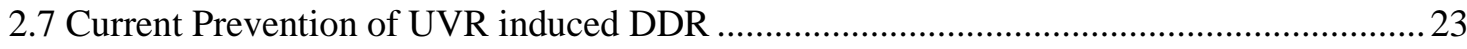

2.7.1 Sunscreen Active Ingredients and Limitations ............................................................. 23

2.8 Sphingomyelin as a Natural Alternative to Sunscreen....................................................... 24

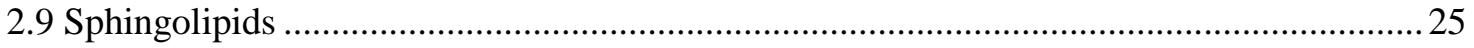

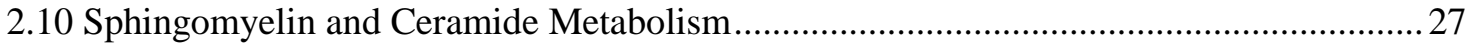

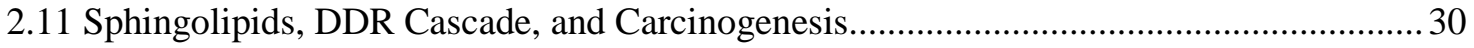

2.12 Bovine Milk Sphingomyelin Past Research and Limitations ........................................... 32

2.13 Probing In Vitro Lipid Dynamics with Fluorescence ...................................................... 33

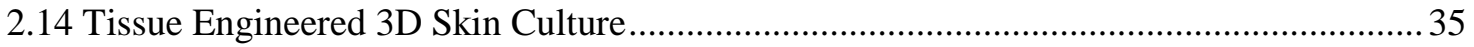




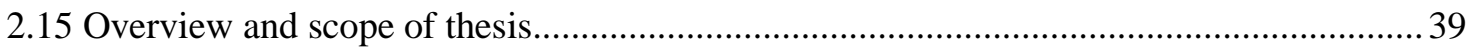

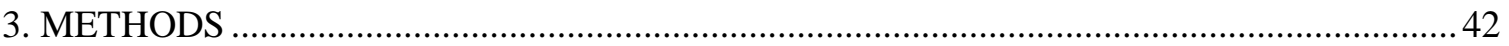

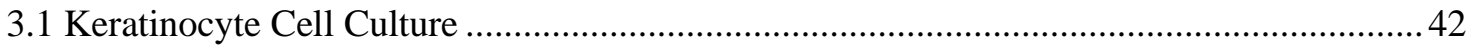

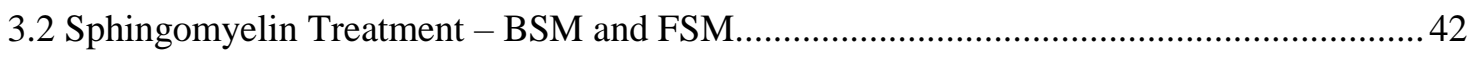

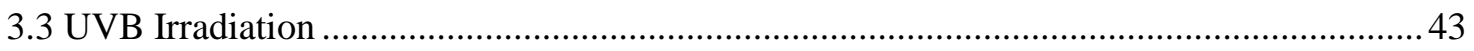

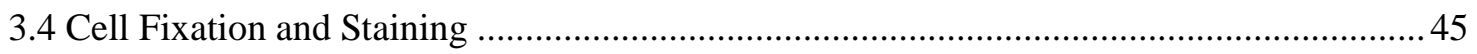

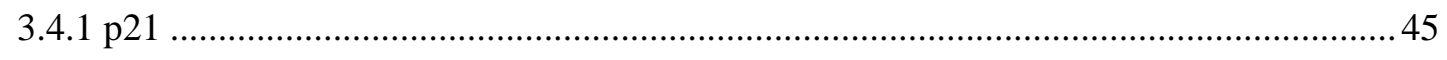

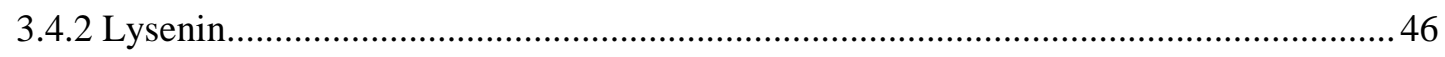

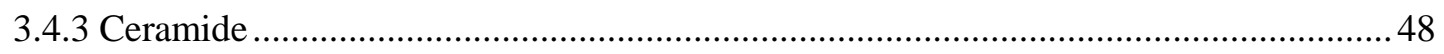

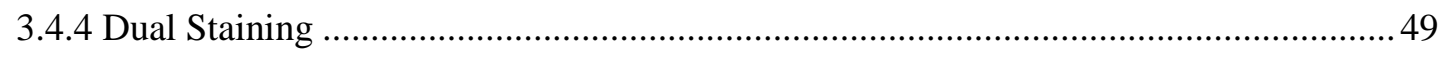

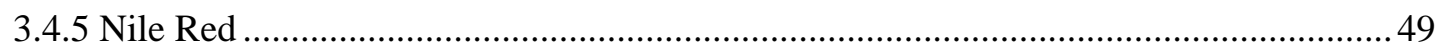

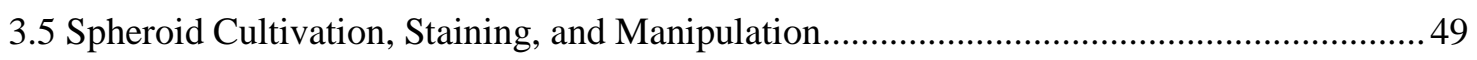

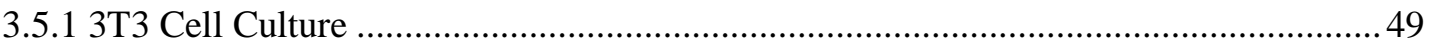

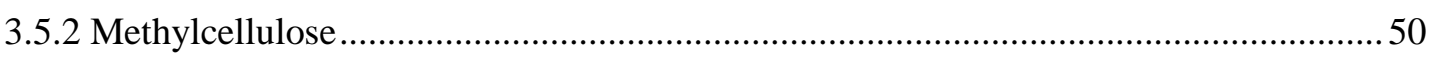

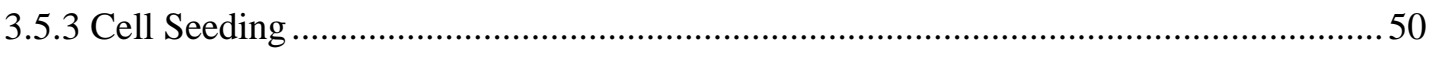

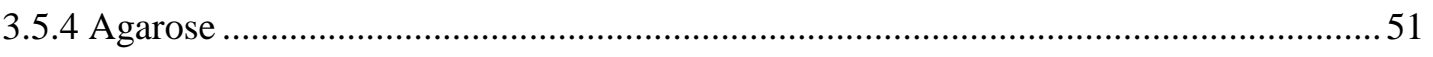

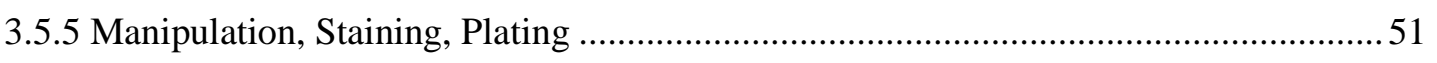

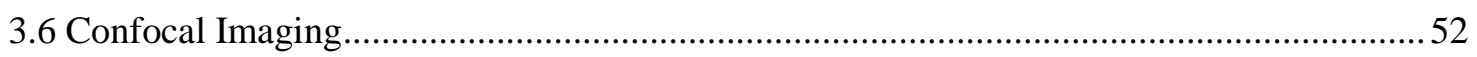

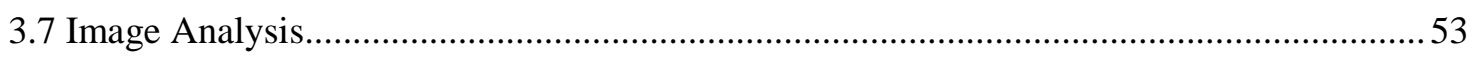

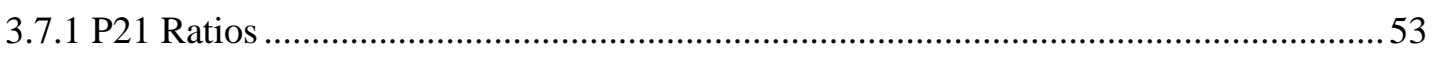

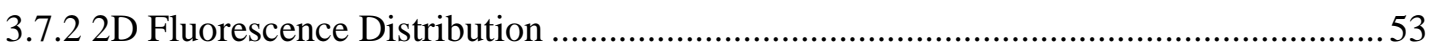

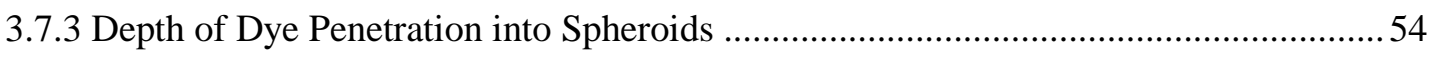

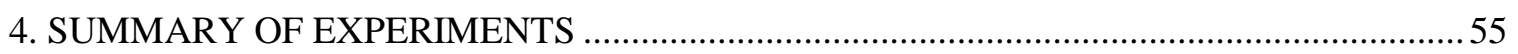

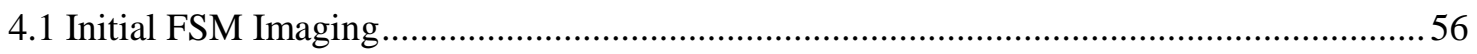

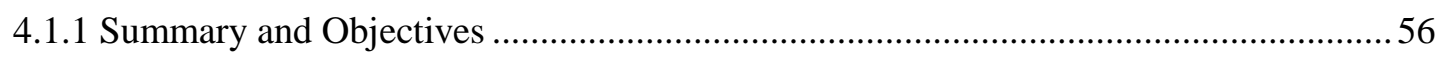

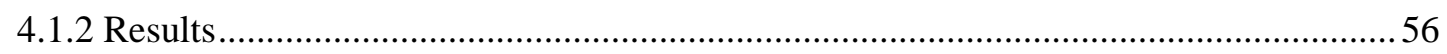

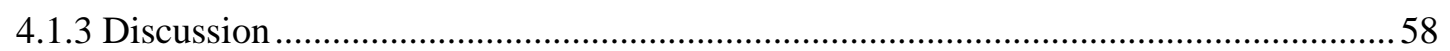

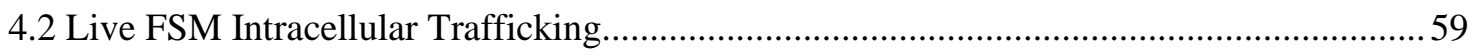

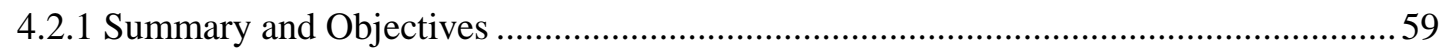

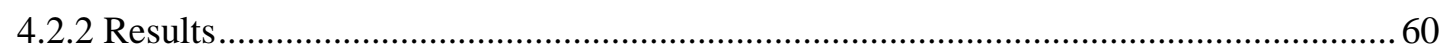

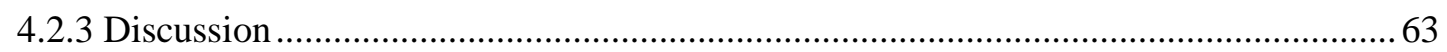

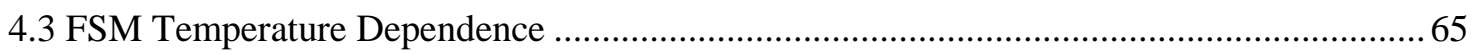

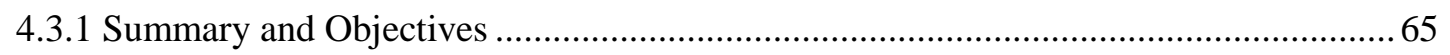

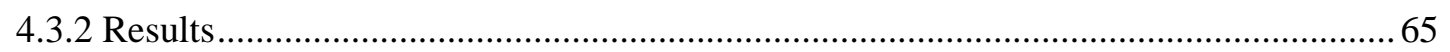

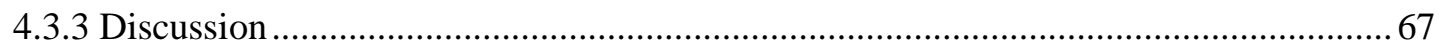




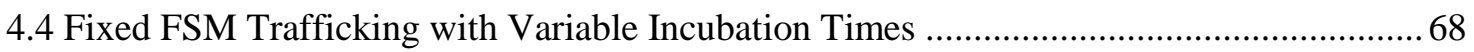

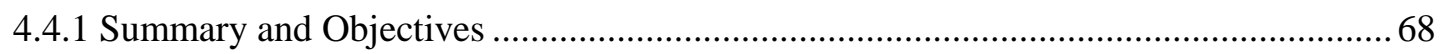

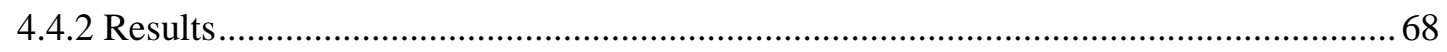

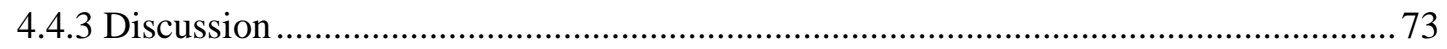

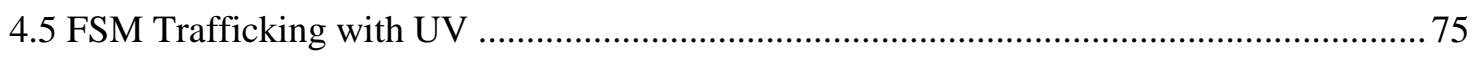

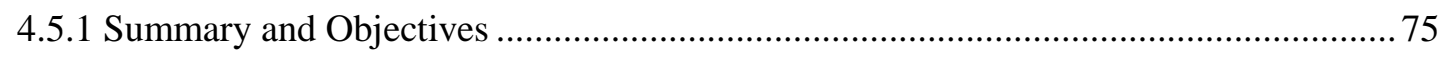

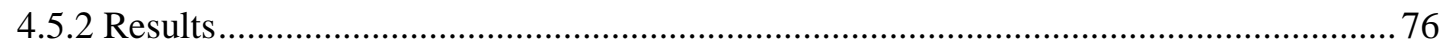

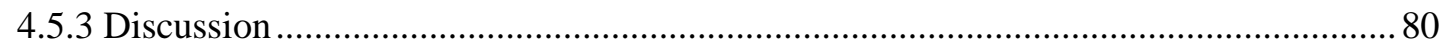

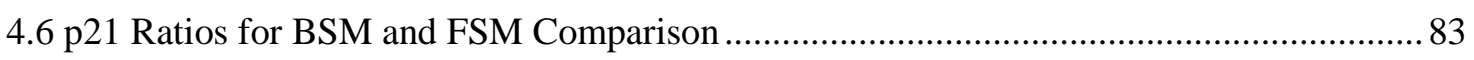

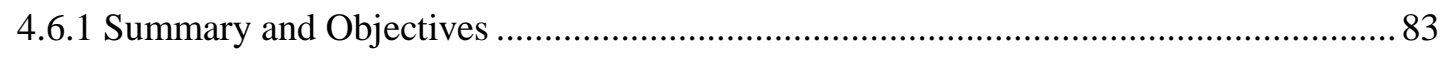

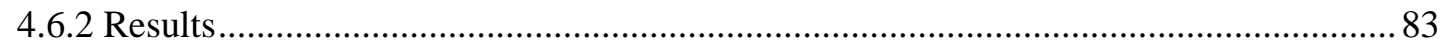

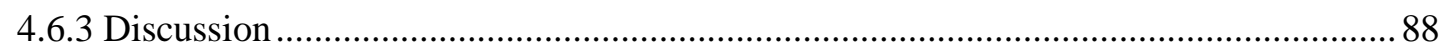

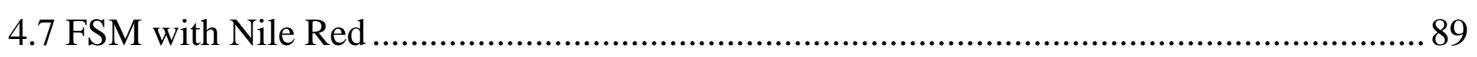

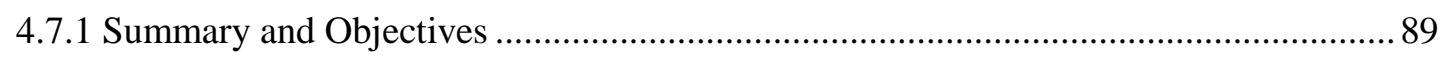

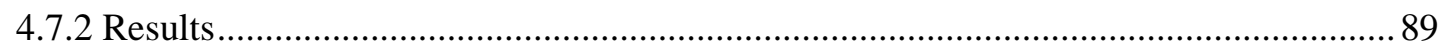

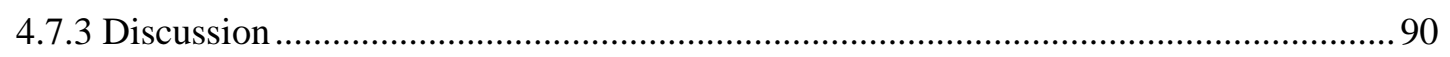

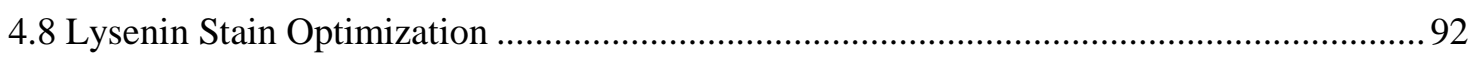

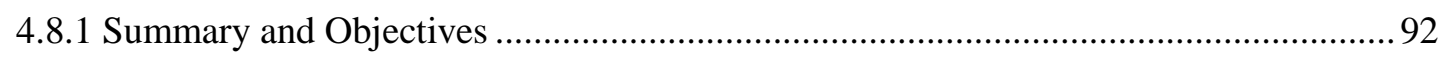

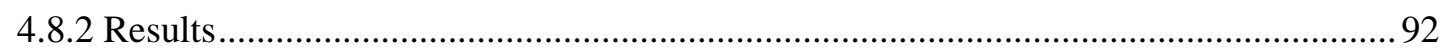

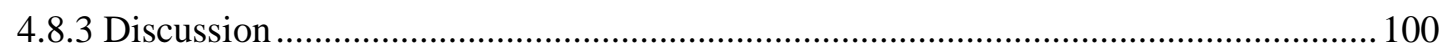

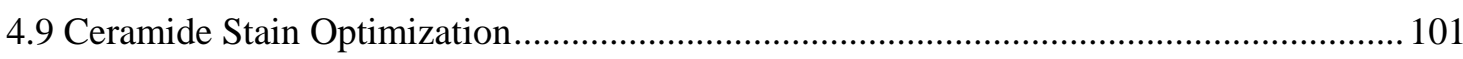

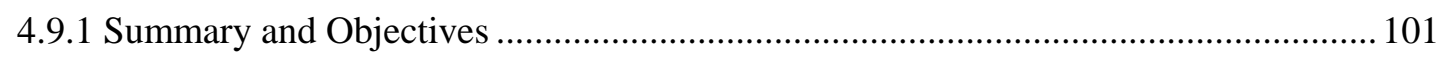

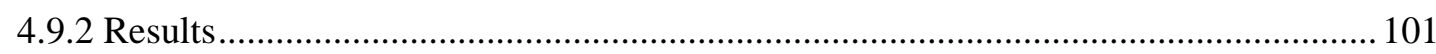

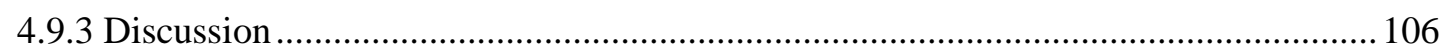

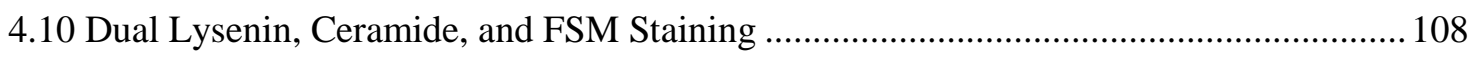

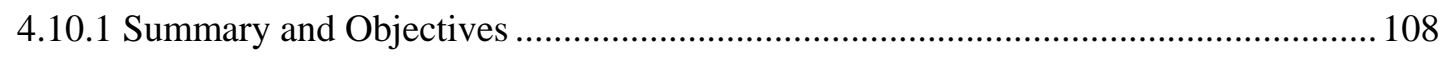

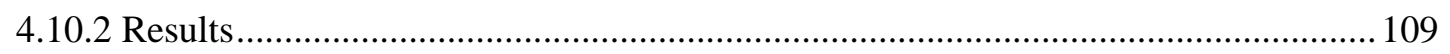

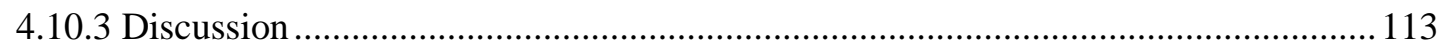

4.11 Troubleshooting FSM Degradation Observed after Dual Immunofluorescence ............. 114

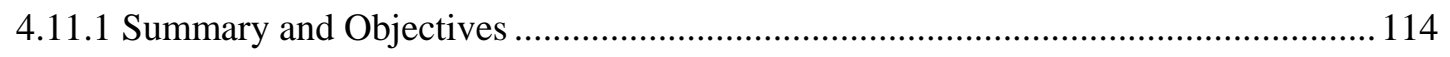

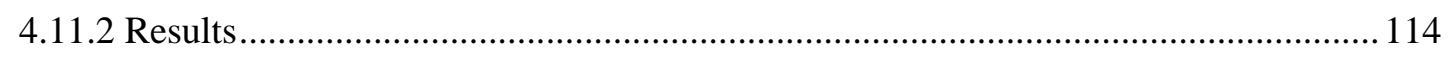

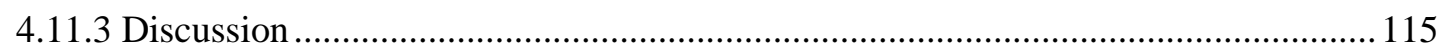

4.12 Dual Lysenin, Ceramide, and FSM Fluorescence Trafficking .................................... 117

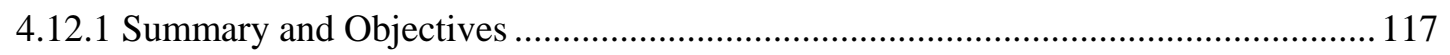

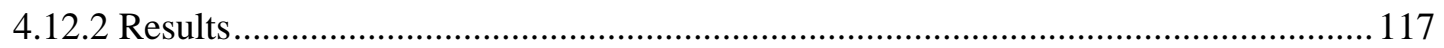




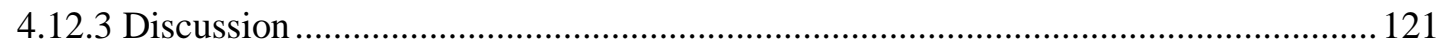

4.13 Spheroid Trial 1: KRTs and 3T3 Single Culture in Non-Adherent U-Wells................... 123

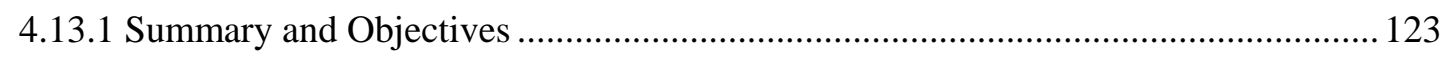

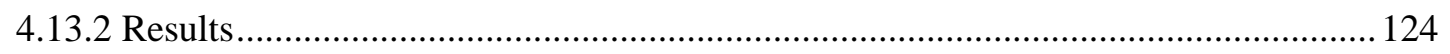

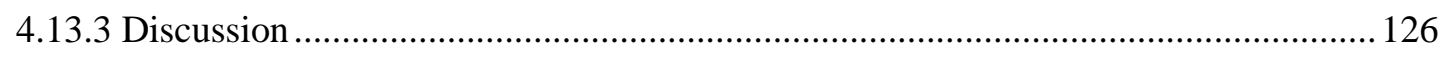

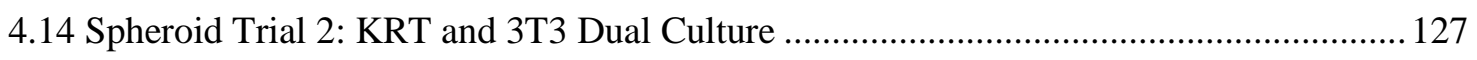

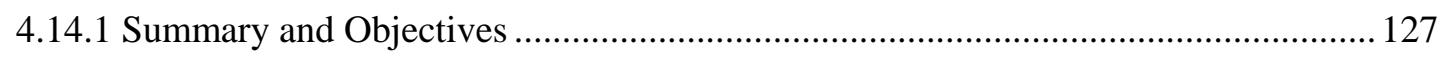

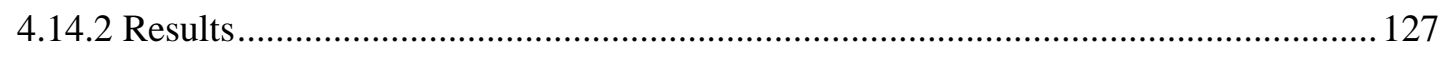

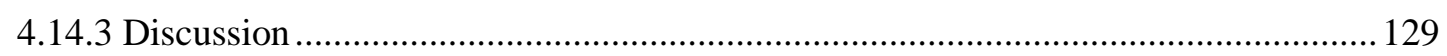

4.15 Spheroid Trial 3: Culture with Agarose-coated Flat Bottom Wells with Triton X and

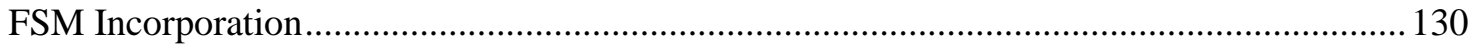

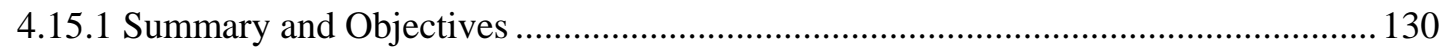

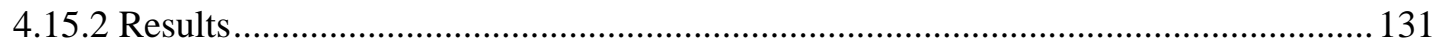

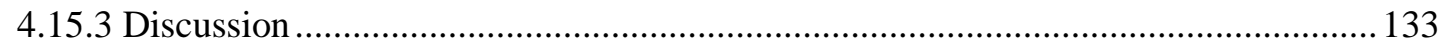

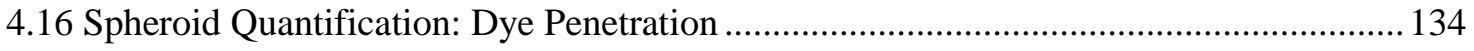

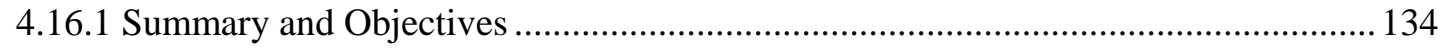

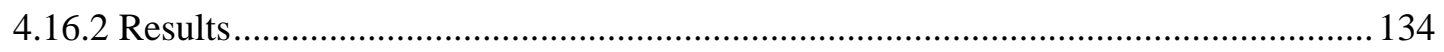

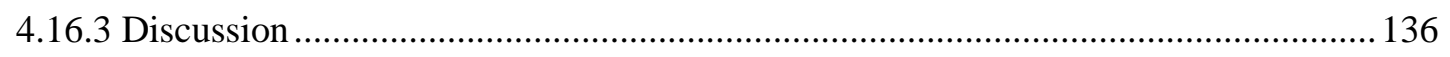

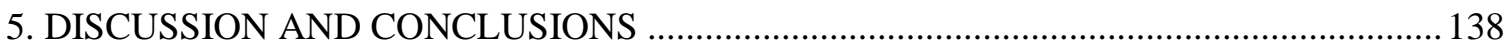

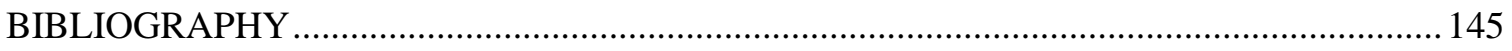
APPENDICES

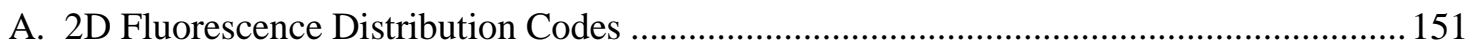

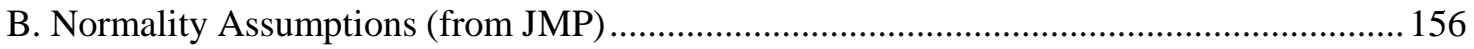




\section{LIST OF TABLES}

Table

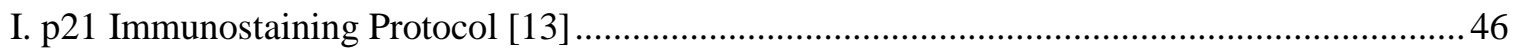

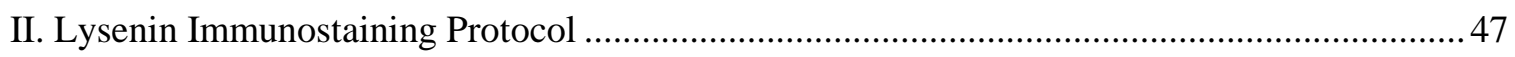

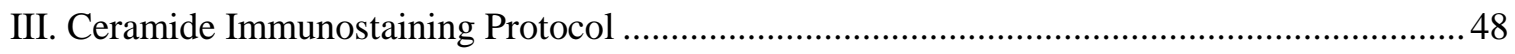

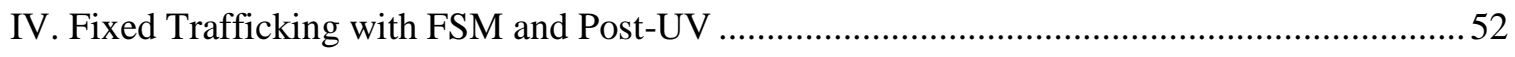

V. Dual Staining with FSM, Lysenin, Ceramide …..............................................................52

VI. Thesis Goals for Characterizing FSM, Staining Protocols, and 3D Cell Spheroids ................55

VII. Averages for All Treatments Demonstrating Trends ....................................................... 88

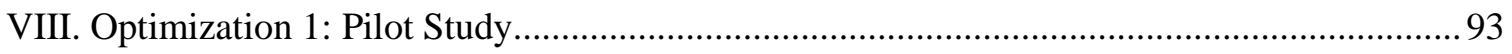

IX. Optimization 2: Comparison of Primary Concentrations .................................................. 96

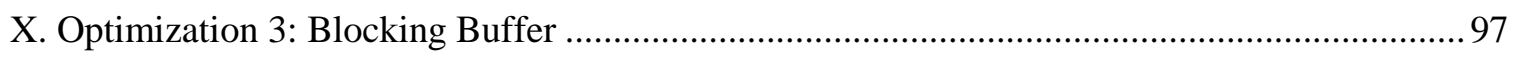

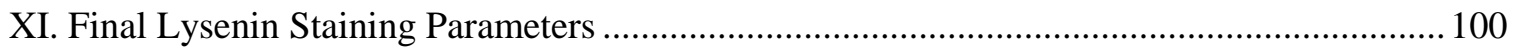

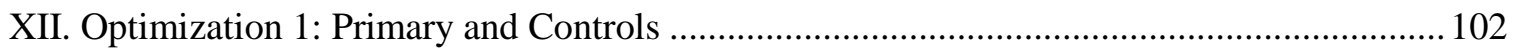

XIII. Blocking to Reduce Non-Specific Secondary Binding ................................................ 104

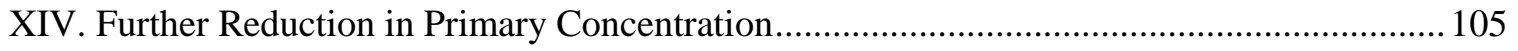

XV. Optimized Ceramide Immunofluorescence Parameters ................................................... 106

XVI. Experimental Parameters Varied for Dual 3T3 KRT Spheroids...................................... 127

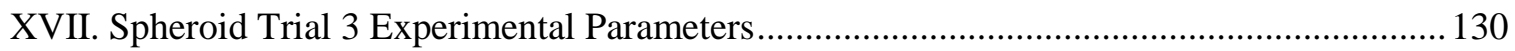


Figure

\section{LIST OF FIGURES}

1: The Three Layers of the Skin and Associated Accessory Organs [15].................................... 4

2: Layers of the Skin Epidermis [15]. The layers of the skin from most superficial to deep are Stratum corneum, Stratum lucidum (absent in thin skin), Stratum granulosum, Stratum spinosum, and Stratum basale.

3: Types of BCC Tumor Skin Growths [5].(A) Open Sore. (B) Red Patch of Irritated Skin.

(C) Shiny Bump or Nodule. (D) Pink Growth. (E) Scar-Like Area.

4: Types of SCC Tumor Skin Growths [5]. (A) Scaly red patch. (B) Elevated growth.

(C) Persistent open sore that bleeds. (D) Wart-like growth.

5: Differing UV Wavelengths (UVA, UVB, UVC) and Skin Penetration Depths [40].

UVA and UVB are wavelengths of primary concern for skin cancer. UVA radiation penetrates deeper into tissue while UVB is absorbed by the epidermis. UVB is responsible for DNA damage.

6: DNA Mutations because of UV [18].(A) T-T adjacent bases. (B) T-C adjacent bases.

(C) After UV, a cyclobutene pyrimidine dimers (CPD) forms. (D) After UV, a (6-4) photoproduct forms.

7: UV-Induced DNA Damage Response and Cell Pathways [6].The goal is to maintain genomic DNA identity. DNA damage initiates ATM/ATR proteins which signal to p53 and CHK1/2 to either induce apoptosis, DNA repair, or cell cycle arrest.

8: p53 Regulation Leading to Apoptosis and Cellular Senescence [43]. P53 increases

WAF1 and BAX leading to either apoptosis or cellular senescence.

9: Normal Cell Cycle [44].The cell cycle consists of G1, S, G2, and Mitosis with various checkpoints.

10: Etiology and Progression of Cancer [20].Cancer results from repeated genetic alterations.... 22

11: General Chemical Structures for Sphingomyelin, Ceramide, Sphingosine, and Sphingosine-1-Phosphate [48].(A) Sphingomyelin, (B) Ceramide, (C) Sphingosine, (D) Sphingosine-1-Phosphate (S1P).

12: Pathways of Ceramide Generation and Metabolism [53]. Pathway 1 represents de novo synthesis, pathways labeled 2 are complex sphingolipid hydrolysis to ceramide, and pathway 3 is the salvage pathway.

13: Summary of Sphingolipid Metabolism and Location in a Cell [27].Ceramide is metabolized to sphingomyelin and S1P in different cellular locations.

14: Ceramide Mechanisms for Cellular Apoptosis [55]. Ceramide and S1P mediate contradictory cell functions

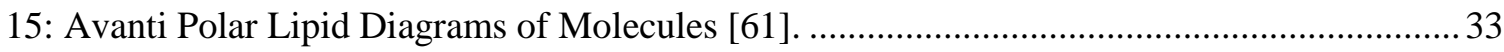

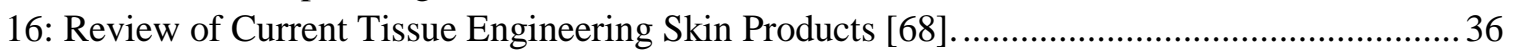

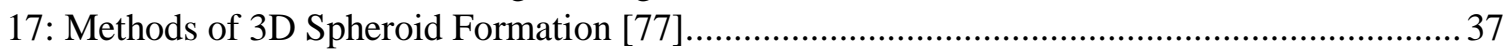

18: UVB Radiation. (A) Calibration of Lamp, sensor (white arrow), power meter (red arrow), ABS plastic tray (green arrow). The white UV lamp is shown atop the black containment box. The exposure time was calculated based on the desired dose and average intensity of the lamp. (B) UV calibration and treatment to cells were conducted inside the culture hood after spraying all equipment down with $70 \%$ IPA. The cells were placed on the blue plastic tray and slid under the UV box/lamp. Spent media was replaced prior to UV... 43 
19: Typical Plate Distribution for SM and UV Treatments.SM was administered in a diagonal pattern with (+) meaning the presence of SM and (-) denoting the absence of SM. The entire plate was subjected to UV, as indicated by the lightning bolt.

20: First FSM Study. (A) Fixed cells at 40x objective demonstrating that FSM signal survives PFA fixation, but a higher objective is a better indicator of intracellular fluorescence distribution. (B) Live cells at 100x objective showing blebbing (white arrow) from endocytosis/exocytosis, a mechanism for cellular FSM entry/exit. (C) Time image of live cells at 100x objective. FSM is predominantly concentrated in the plasma membrane, with some intracellular accumulation. (D) Time image, 100x objective, with intracellular distribution (white arrow) of FSM into cells.

21: Representative Images for Live FSM Trafficking with Variable Incubation Times. (A) 10 Minutes (100x), (B) 1 Hour (100x), (C) 12 Hours (100x). No difference in trafficking was observed between all timepoints, suggesting endocytosis and exocytosis homeostasis is reached early in the cell. The plasma membranes had a "shaggy" or coarse FSM staining because cells were directly probed during FSM media incubation and a wash step with PBS was not performed prior to imaging.

22: Three Hour Incubation Live Cells with FSM and DIC. Representative image of all timepoints (10 minutes -24 hour incubation) at 100x objective. Relatively consistent staining was observed among all timepoints. Intracellular fluorescence was concentrated around the nucleus in what appeared to be Golgi bodies (black arrow). Inset is an overlay of AF 488 (A) and DIC (B - whitelight).

23: Severe Photobleach of Live FSM Timescans. (A) First image of live timescan. (B) Last image of live timescan (after 5 minutes with 5 seconds between laser pulses) showing photobleaching, especially evident in the intracellular space. (C) Overlay of first image with pseudo-colored blue last image. The blue signal plasma membrane forms a halo around the green plasma membrane, suggesting the cell is pulling away from the plate and shifting upward in the z-axis.

24: Profile Plot of Signal Degradation and Cell Movement Over Time. The profile plot was constructed from the line drawn across the image in Figure 22. Green represents the first image and blue the last image in the scan. (A) intense plasma membrane localization, (B) intracellular fluorescence clusters near the Golgi elicit small spikes in fluorescence, (C) lack of signal in the nucleus. The blue plot has a significantly lower mean gray value and is shifted outward compared to the green line

25: Rapid FSM Entry and Temperature Dependence. (A-D) FSM after approximately 90 minutes (100x objective). Heterogeneous staining is apparent. Some cells start rapidly accumulating FSM, but uptake is highly variable among cells. (E-H) Representative time images between $\mathrm{C}$ and $\mathrm{D}$ panel of plasma membrane blebbing and release of FSM into extracellular space after 90 minutes. 100x objective.

26: Fixed FSM Trafficking with Variable Incubation Times. (A) 10 min, (B) 1 hour, (C) 12 hour, (D) 24 hour, (E) Profile plot line draw across the boxed cell for the 10 minute treatment. (F) Corresponding profile plot. Red and black arrows denote intracellular and plasma membrane fluorescence respectively. Fluorescence localization and brightness appears to remain consistent regardless of incubation time. 100x objective.

27: Example Workflow of 2D Fluorescence Distribution. (A-C) Profile Plots corresponding to the lines in (D). For each cell within the image, an ROI was drawn to encompass the cytoplasm and exclude the nucleus (purple outline). Lines extending radially from the centroid were formed and rotated $360^{\circ}$ at $6^{0}$ increments. In total, 60 profile plots per cell 
were generated. The profile plots along each line were averaged, then the distance was normalized in MATLAB by linear interpolation between points.

28: FSM Trafficking with Different Continuous Incubation Times $(n=6)$. Standard error bars are represented.

29: Relative Amount of FSM in a Cell over Various Timepoints ( $n=6)$. \# is $p<0.05$ for pairwise comparisons. $\$$ is $p<0.05$ compared to global mean. No other significant pairwise differences between the groups according to Tukey HSD. Standard error bars are represented.

30: Troubling ROIs. (A) Inaccurate cellular outlines encompassing localized bubbles and blebs (white arrow). (B) Potential cell area data bias. 100x objective.

31: Fluorescence Distribution for 1 Hour Incubation ( $n=6$ wells). Control (no UV) for the 24 hour timepoint is denoted in black. All post-UV timepoints exhibit similar mean gray values and are grouped close together, with the exception of the no UV control with demonstrates a visible reduction in average mean gray value around the nucleus and throughout the cell. Standard error bars are represented.

32: Fluorescence Distribution for 24 Hour Incubation ( $n=4$ wells). Control (no UV) for the 24 hour timepoint is denoted in black. All post-UV timepoints have similar mean gray values throughout the cell and are grouped close together including the no UV control. Standard error bars are represented.

33: Comparison of Post-UV Fluorescence Distributions for 1 Hour and 24 Hour FSM Incubation. The 1 hour FSM incubation (black curve) had a consistently larger mean gray value compared to 24 hour FSM incubation (gray curve) along the entire cell distance from the nucleus. Standard error bars are represented.

34: Relative Amount of Cytoplasmic Fluorescence for Various Times after UV.\#, \$, \%, $\varepsilon$, *, $\&$ denote statistically different comparisons between the two with the same symbol $(\mathrm{p}<0.05)$. Standard error bars are represented.

35: No UV with BSM and FSM Treatment.(Top Row) BSM (-), UV (-). ( $2^{\text {nd }}$ Row) BSM (+), UV (-). ( $3^{\text {rd }}$ Row) FSM (-), UV (-). (Bottom Row) FSM (+), UV (-). Images demonstrate relatively low p21 nuclear expression (white arrow) suggesting that SM incubations are not cytotoxic. 40x objective.

36: UV with BSM and FSM Treatment. (Top Row) BSM (-), UV (+). ( $2^{\text {nd }}$ Row) BSM (+), UV (+). ( $3^{\text {rd }}$ Row) FSM (-), UV (+). (Bottom Row) FSM (+), UV (+). Reduced amount of nuclear p21 levels (white arrow) are apparent in the SM treatment cases compared to control no SM treatments. 40x objective.

37: p21 Ratio Results (n=4 wells).(A) BSM p21 Ratios. (B) FSM p21 Ratios. (C) Comparison Between Bovine Milk SM and C6-NBD SM p21 Ratios for UV Cases. Standard error bars are represented.

38: Nile Red Representative Images. A-D Nile Red and FSM Dual Staining (100x objective) (A) Merge of Nile Red, FSM, and Hoechst. (B) Hoechst, (C) FSM, (D) Nile Red. E-H Nile Red Only (100x objective) (E) Merge, (F) Hoechst, (G) AF 488 channel (no FSM), (H) Nile Red. Some bleed through was observed from Nile red into the AF488 channel. With the addition of exogenous FSM, the brightness of the Nile red increased in (D) compared to $(\mathrm{H})$.

39: Control Images. (A-C) $\mathrm{H}+1+2$ demonstrating non-specific primary binding (60x objective). (A) Merge, (B) DAPI, (C) AF 488. (D-F) H+L+2 demonstrating slight nonspecific secondary fluorescence (60x objective). (D) Merge, (E) DAPI, (F) AF 488. 
40: Experimental H+L+1+2 Images. (A-B) Two representative merge images of lysenin $(1 \mu \mathrm{g} / \mathrm{mL})$ and primary (1/1000). (C-D) Two representative merge images of lysenin $(1 \mu \mathrm{g} / \mathrm{mL})$ and primary $(1 / 500)$. (E) Representative merge image of lysenin $(1 \mu \mathrm{g} / \mathrm{mL})$ and primary (1/300). (F) Representative merge image of lysenin $(3 \mu \mathrm{g} / \mathrm{mL})$ and primary (1/300). 60x objective.

41: $1 / 1000$ versus $1 / 500$ Primary Dilution Comparison. (A) $\mathrm{H}+1+2$ merge with primary (1/1000). (B) H+1+2 merge with primary (1/500). Insets zoomed-in views of (A) and (B) are included in the right panel. 100x objective.

42: Blocking Buffer Optimization Non-Specific Binding for $\mathrm{H}+1+2$ Control.

(A) 4\%BSA/Goat, (B) 4\%BSA/Goat+PBS-Tween, (C) 4\%Milk/Goat+PBS-Tween,

(D) No Blocking. 100x objective. .98

43: H+L+1+2 Staining Efficiency Comparisons During Optimization Steps. (A-B) 1/1000

Primary optimization comparison demonstrating incubating for 1 hour. (A) results in a similar staining efficiency as the 9 hour incubation (B). (C-F) Blocking optimization images (60x objective) demonstrating that all blocking varieties yielded similar images. 100x objective.

44: Control Merged Images with DAPI. (A) H+2 control exhibits non-specific secondary binding, (B) $\mathrm{H}+1$ control shows no random, unattributable fluorescence of the primary or Hoechst into the AF 647 ceramide channel. 100x objective.

45: Experimental $\mathrm{H}+1+2$ Images. (A-B) Two representative merge images of primary (1/200) for 1 hour. (C-D) Two representative merge images of primary (1/200) and 9 hours. (E-F) Two representative merge image of primary (1/300) for 1 hour. (G-H) Two representative merge image of primary (1/300) for 9 hours. Within each treatment, inconsistent ceramide results were observed. Some cells had diffuse ceramide staining, while others exhibited pockets of intense, bright staining. 100x objective.

46: Control H+2 with 4\% BSA/4\% Goat Serum Blocking Merge Image with DAPI. No nonspecific secondary staining is observed. 100x objective.

47: $1 / 300$ versus 1/400 Ceramide Primary Comparison. (A)1/300 dilution (60x objective) demonstrating diffuse staining. (B) 1/300 dilution (40x objective) with patchy staining. (C) 1/400 dilution (60x objective) with diffuse staining. (D) 1/400 dilution (60x objective) with patchy staining. The ceramide stain again displayed inconsistent cytoplasmic expression.

48: 2D and Maximal Z-Projections of FSM and Lysenin Immunofluorescence. (A) 2D image of AF 594 lysenin channel. Staining mostly concentrates along the plasma membrane (B) Z-projection of lysenin channel. Adding the 3D component to the images better represents lysenin distribution. (B) inset shows some regions of concentrated lysenin fluorescence (C) Merge 2D image AF 594 lysenin with AF 488 FSM. (D) Merge z-projection of lysenin and FSM. 100x objective.

49: 2D and Maximal Z-Projections of FSM and Ceramide Immunofluorescence. (A) 2D image of AF 488 FSM channel. (B) Z-projection of AF 488 FSM channel.

(C) 2D image of AF647 ceramide. (D) Z-projection of AF 647 ceramide channel. 100x objective.

50: Differential Staining of Lysenin and Ceramide. (A-C) Bright plasma membrane lysenin staining as demonstrated in the z-projection (A), 2D image (B), and orthogonal view (C) which shows distinct fluorescence far from the nucleus (white arrow). (D-F) Bright lysenin staining directly around the nucleus as demonstrated by the z-projection (D), 2D image (E), and orthogonal view (F) which shows distinct fluorescence only 
close to the nucleus (white arrow). (G-I) Distinct plasma membrane and intracellular ceramide staining as demonstrated in the z-projection $(\mathrm{G}), 2 \mathrm{D}$ image $(\mathrm{H})$, and orthogonal view (I) which shows fluorescence clustered around the nucleus (white arrow). (J-L) Absence of a definitive ceramide fluorescence signal as demonstrated in the z-projection (J), 2D image (K), and orthogonal view (L) which only shows a slight signal above the nucleus (white arrow). 100x objective.

51: Z-Projection of FSM, Lysenin, and Ceramide after Dual Immunostaining. (A-C) 60x objective image. (A) FSM demonstrating significant signal degradation, (B) Lysenin, (C) Ceramide. (D-F) 100x objective. (D) FSM, (E) Lysenin with pocket of weakly stained cells, (F) Ceramide.

52: Signal Degradation from Various Staining Treatments. (A) Control, (B), Triton X permeabilization with PBS washes, (C) Triton X permeabilization with $0.05 \%$ Tween-20 Washes. 100x objective.

53: Additional Signal Degradation Demonstrations. (A) Dual immunofluorescence FSM channel imaged using trafficking settings. (B) Normal FSM with no extra treatment imaged using trafficking settings (no dual staining). (C) Same FSM image but with dual immunofluorescence settings. 100x objective.

54: Representative Dual Immunofluorescence 2D Images for Various Continuous FSM Incubation Times. 100x objective.

55: FSM, Lysenin, and Ceramide Fluorescence Distributions over Variable Continuous Incubation Times ( $\mathrm{n}=4$ wells). (A) FSM, (B) Lysenin, (C) Ceramide.

56: Relative Amounts of Ceramide, FSM, and Lysenin. No significant differences among ceramide and FSM timepoints. \# significant difference from global mean. * significant difference from each other. Standard error bars are represented.

57: Whitelight Images of 3T3 and KRT Spheroids after 2 Days for Culture. (A) 3T3 cells formed a distinct spheroid. (B) KRT cells failed to form spheroids and instead small clusters of cells were visible (e.g. black arrow).

58: Representative Images of KRT and 3T3 Spheroids. (A) KRT cells failed to form spheroids. (B) $3 \mathrm{~T} 3$ spheroid. (C) Orthogonal view of 3T3 spheroid showing that the Hoechst dye did not penetrate into the depth of the spheroid. (D) 2D image of start of z-stack, (E) 2D image of middle of z-stack, (F) 2D image of end of z-stack demonstrating the lack of Hoechst dye visibility at the interior core of the spheroid. 40x objective.

59: Whitelight Images after 3 Days of Culture. (A) 2:1 40\%. (B) 1:1 40\%. (C) KRT only $40 \%$. Satellite colonies of KRTs are observed surrounding the main spheroid (arrow). No spheroids formed in the KRT only culture..

60: Z-Projections of Confocal Spheroid Z-Stacks. (A) 3T3: KRT at 1:1 ratio with 1,000 cells total and 40\% methylcellulose (B) 3T3: KRT at 1:1 ratio with 5,000 cells total and 40\%, methylcellulose (C) 3T3: KRT at 2:1 ratio with 5,000 cells and 20\% methylcellulose (D) 3T3: KRT at 2:1 ratio with 5,000 cells total and 40\%, methylcellulose (E) 3T3: KRT at 1:2 ratio with 5,000 cells total and $40 \%$ methylcellulose. 40x objective.

61: Agarose is Necessary and Sufficient to Create a Non-Adherent Environment for Spheroid Formation of 3T3 Cells. (A) Agarose, (B) No Agarose, (C) Agarose Meniscus (black arrow).

62: Orthogonal View of 3D Spheroid with FSM and Triton X. (A) Representative FSM spheroid z-project. (B) Start of z-stack for representative FSM spheroid.

(C) Corresponding orthogonal view. (D) Middle of z-stack for representative FSM 
spheroid (E) Corresponding orthogonal view. (F) FSM 1 hour, no Triton X (G) FSM 24 hours, no Triton X (H) FSM 24 hours, Triton X. 40x objective.

63: Analysis Method for Spheroids. (A) Slice in Spheroid, (B) Binary, (C) Z-projection with outlines, (D) 1/10 division, (E) 3/10 division, (F) 8/10 division. (G) Graphical representation of the fluorescence distribution.

64: Relative Amount of Hoechst Dye over Normalized Spheroid Depth. Standard error bars are represented. 


\section{INTRODUCTION}

The skin is one of the largest organs in the human body and provides a physical barrier while regulating temperature and water content, among many other crucial functions [15], [16]. Non-Melanoma Skin Cancer (NMSC) affects approximately 3.3 million Americans each year and is currently the most commonly diagnosed disease [1], [2], [5]. The common forms of NMSC are Basal Cell Carcinoma (BCC) and Squamous Cell Carcinoma (SCC), with BCC representing about $80 \%$ of NMSCs [2], [17] Given the prevalence of NMSC in the human population, understanding the cause and potential treatments is crucial.

Ultra Violet Radiation (UVR) is mainly responsible for damage to skin and NMSC occurrence [4]. UVB light $(280-320 \mathrm{~nm})$ is the most damaging to skin and causes direct damage to Deoxyribonucleic Acid (DNA) in the form of cyclobutene pyrimidine dimers and (6-4) photoproducts which disturb the double helix structure [3], [4]. These two photoproduct lesions can lead to further DNA mutations like CC->TT base substitutions predominantly referenced as a 'UV signature mutation' [18], [19]. Mutations in oncogenes like the p53 tumor suppressor gene increase the rate of developing cancer such as NMSC [18].

Cells directly detect UV DNA damage with molecular sensors like Mre11, NBS1, and Rad 50 which initiate the DNA Damage Response (DDR), a group of signaling pathways which lead to either apoptosis, DNA repair, or cell cycle arrest in order to maintain genome integrity [6]. The tumor suppressor protein p53, also coined the 'guardian of the genome', serves as a transcription factor to regulate genes involved with cell cycle arrest and apoptosis [7], [8]. For example, p53 activates downstream p21 protein which is a cyclin-dependent kinase inhibitor that arrests DNA replication to allow time for DNA repair [9]. Skin carcinogenesis can develop when DNA mutations cause loss-of-function mutations in the p53 tumor suppressor gene leading to cell evasion of normal DNA repair and apoptosis processes [18], [20]. Repeated exposures to 
environmental triggers (e.g. solar UVR) can cause a progression of NMSC cancer from premalignant lesions to tumors and eventually metastatic lesions [20].

Current methods to avoid NMSC include protective actions like avoiding sun, wearing protective clothes, and regularly applying sunscreen [12], [17]. Sunscreen is an essential component of photoprotection, but according to a 2016 poll of US dermatologists, $99 \%$ believed their patients did not apply enough sunscreen or use it according to product guidelines [21]. Furthermore, some controversy exists around certain chemical active ingredients such as oxybenzone [10]-[12]. Not only has research demonstrated that oxybenzone pollutes the environment, a 2001 animal study demonstrated endocrine disruption [10], [12]. In addition, a 10year retrospective study of 24,000 patients asserted that $70.2 \%$ of patients confirmed positive for oxybenzone allergies when patch tested [12]. Given the prevalence of skin cancer and controversy over the systemic safety of oxybenzone, the need for safer, more natural alternatives is of scientific importance.

Bovine milk sphingomyelin (BSM) has demonstrated photoprotective potential [13], [14], [22], [23]. Orally administered BSM in mice accumulated in the stratum corneum layer of the skin and reduced transepidermal water loss after UVB radiation [22], [23]. Furthermore, research by De Guzman and Campbell have demonstrated that BSM administration to a monolayer of keratinocyte (KRT) skin cells reduced p21 and p53 levels after UVB compared to the control [13], [14]. The decrease in both p21 and p53 DDR proteins was correlated with an increase in photoprotection offered by BSM to KRT cells.

The success of BSM treatment in UVB KRT models has prompted research into the reason for the observed photoprotection. Sphingomyelin (SM) is normally degraded to ceramide in the plasma membrane by SMase enzymes [6]. Furthermore, ceramide levels have been demonstrated to increase after UVB radiation in KRTs which offers photoprotection by increasing the apoptosis rates of potentially damaged cells [24]-[26]. A current hypothesis for 
SM photoprotection is that exogenous addition to cells increases the amount of ceramide production through hydrolysis mechanisms and subsequently increases rates of apoptosis in UV exposed cells. However, the levels of intracellular SM after UVB irradiation in KRTs is less characterized, and no groups combine exogenous SM treatment with UVB to quantify changes in intracellular SM levels. Quantifying intracellular SM could answer questions about lipid homeostasis, trafficking, and mechanisms of photoprotection in a cell. Additionally, fluorescence imaging methodologies to quantify cellular localization were not employed in past BSM research [13], [14]. Therefore, the goals of this thesis were to characterize Fluorescent SM (FSM) as an analogue to BSM and assess the changes in intracellular FSM distribution after different incubation times and after UV. Different metabolic processes occur in different locations in a cell (plasma membrane, golgi, endoplasmic reticulum) and trafficking fluorescence location elucidates mechanisms of metabolism and degradation in a cell [27]. Two immunofluorescence stains for endogenous SM (lysenin) and ceramide were also optimized and used for a preliminary fluorescence distribution studies to characterize FSM with colocalization in the future. Finally, the use of spheroids as a more relevant 3D model was assessed by characterizing NIH 3T3 mouse fibroblasts as proof-of-concept. 


\section{BACKGROUND}

\subsection{Integumentary System}

The skin is an organ member of the Integumentary System and primarily forms a barrier to protect the body from damage and disease [15]. In adult humans, skin and accessory organs comprise approximately 16 percent of body weight and cover about $2.0 \mathrm{~m}^{2}\left(22 \mathrm{ft}^{2}\right)$ of surface area, making the Integumentary System the largest organ system in humans [15], [28]. Exhibiting a multilayered and complicated structure, the Integumentary System performs numerous functions crucial for overall health.

The skin consists of two main layers, an epidermis and an underlying dermis (Figure 1). Directly beneath the dermis is the hypodermis, also called the subcutaneous, subcutis, or superficial fascia [15], [28].

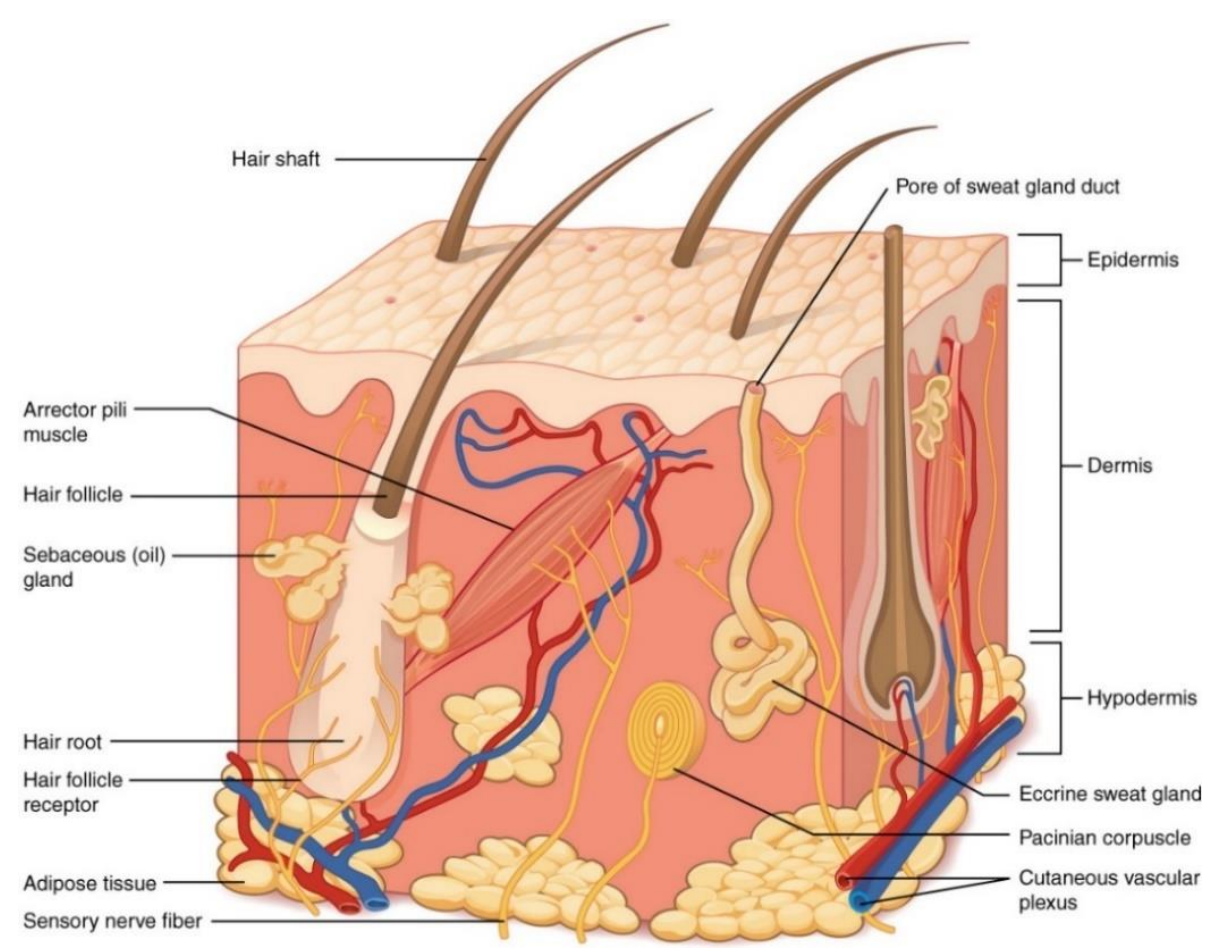

Figure 1: The Three Layers of the Skin and Associated Accessory Organs [15]. 
Skin thickness varies from $0.5 \mathrm{~mm}$ lining the eyelids to $4.0 \mathrm{~mm}$ surrounding the heel of the feet [28]. Although its main function is protection from Ultra Violet (UV) radiation, injury, infection, and external stresses, skin also provides thermoregulation, absorption/secretion of water and metabolites, production of vitamin D, and sensation touch and pain [15]. The epidermis also dictates cutaneous movement of water and electrolytes because of its high lipid content and hydrophobic nature [29]. The malfunction of skin in diseases and disorders like cancer demonstrate the essential function of the Integumentary System and the need to cherish it.

Although the focus of this section is the structure and function of the epidermis, the dermis and hypodermis play an integral role in skin health. The dermis is a vascularized and innervated connective tissue layer comprised of connective tissue (collagen and elastin), fibroblasts, sweat glands, and hair [28]. The dermal papillae form the superficial layer and serve as an adhesion site and partition between the epidermis and dermis [28]. Dermal papillae also shunt nutrients from the vasculature to the epidermis which lacks a clear blood supply [28]. The dermis is divided into the superficial region, which contains the dermal papillae and deeper region of collagen and fibroblasts [15], [28]. Beneath the dermis lies the hypodermis, a layer mainly made of adipose tissue and sweat glands [15], [28]. Taken together, the epidermis, dermis, and hypodermis protect the body from external insults, enable sensory function, perform thermoregulation, and synthesize Vitamin D and other essential nutrients [15]. The subsequent sections will focus on the different layers of the epidermis, with an emphasis on the resident keratinocyte cells and lipid metabolism.

\subsubsection{Epidermis}

The epidermis is the most superficial layer of skin and first line of defense between the body and the external environment [15], [28], [30]. About 100 microns thick, this layer is an avascular and self-renewing stratified squamous epithelial cell structure composed of four layers (listed from most superficial to deep): stratum corneum, stratum granulosum, stratum spinosum, 
and stratum basale [15], [28], [30]. In thick callous skin (i.e. lining soles of feet, fingertips or palms of hands), the epidermis has a fifth layer of translucent cells between the stratum corneum and stratum granulosum called the stratum lucidum (Figure 2) [15], [28].

The skin is comprised of three main cell types: keratinocytes (KRTs), melanocytes, and fibroblasts [31]. KRTs are the predominant epidermal cell type comprising 95\% of total cells [30]. KRTs exhibit a differential phenotype depending on the layer [30], [31]. They start off as viable, nucleated, and cobblestone-shaped cells in the stratum basale then undergo remarkable changes leading to the conventional "flaky" stratified cell phenotype in the stratum corneum [30], [31]. KRTs also produce keratin, an intracellular fibrous protein and structural component responsible for the hardness and water-resistance of hair, nails, and skin [32]. The type of keratin produced depends on the layer and KRT differentiation stage [32].

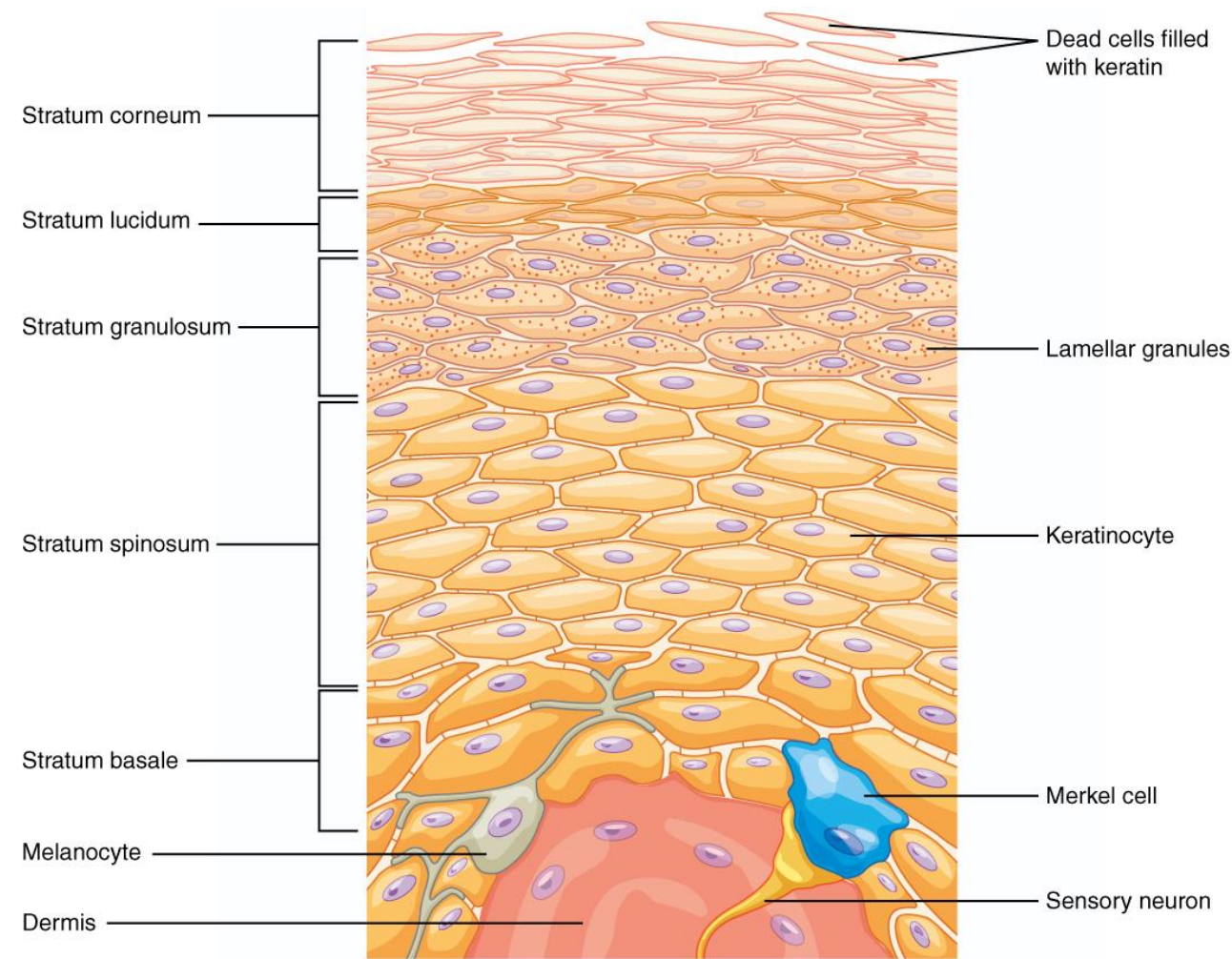

Figure 2: Layers of the Skin Epidermis [15]. The layers of the skin from most superficial to deep are Stratum corneum, Stratum lucidum (absent in thin skin), Stratum granulosum, Stratum spinosum, and Stratum basale. 
KRTs are produced in the basal layer, then undergo keratinization (cornification) as they push up through the layers of skin and ultimately shed as dead skin flakes from the stratum corneum [30]. The process of KRT differentiation in the basal layer and shedding at the corneum is a cyclic process that replaces the epidermis every four weeks [15]. Melanocytes (1-2\% of epidermis cells) and fibroblasts (dermal layer), are also crucial for skin health and cell phenotype [30].

\subsubsection{Stratum Corneum}

The stratum corneum is the most superficial layer of the dermis made up of 15-30 layers of flattened, dead, and densely-packed cornified KRTs [15], [28]. These terminally differentiated KRTs are known as corneocytes because they are flattened and anucleate [15], [16], [28], [30]. Corneocytes, building blocks of the stratum corneum, originate from stratum granulosum KRTs that rise to the surface before undergoing apoptosis in the corneum [16], [30]. Directly surrounding the corneocytes is a hydrophobic extracellular matrix (ECM) made of mostly ceramide, cholesterol, fatty acids, and proteins including filaggrin and keratin [29]. On average, each KRT cell takes two weeks to rise from the basale to corneum, then another two weeks to cornify before flaking off in a process called desquamation [30].

Lipids originate from lamellar body secretions by cells in the stratum granulosum [16], [29]. In a normal keratinocyte, lipids are synthesized during cell differentiation in the granulosum and packaged into lamellar bodies, small bilayer and circular organelles [29]. In the granulosum, the concentration of calcium peaks and drops rapidly in the corneum, triggering the exocytosis of lipid-filled lamellar bodies into the extracellular space of the stratum corneum [16]. The released lipids are subsequently processed and rearranged parallel to the corneocyte membrane to form a cornified envelope [33]. The lipids are further metabolized into ceramide which accounts for over $30-40 \%$ of lipid weight in the corneum [16]. Cholesterol and sphingomyelin are also present in 
the corneum interstitial space [29]. In total, the high lipid content maintains fluid balance by preventing water loss [33]. When the skin barrier is disrupted, lamellar body secretion and sphingolipid metabolism increase to reestablish the hydrophobic barrier [29], [33].

Filaggrin and keratin together compose $80-90 \%$ of protein mass in the epidermis [16]. Filaggrin ensures corneum water retention while maintaining barrier function [34]. Filaggrin also interacts with keratin to form tight bundles and promote cornification [34]. Loss of filaggrin function can lead to skin conditions like dermatitis, and increases UV sensitivity as demonstrated by an in vitro skin model [34].

\subsubsection{Stratum Granulosum and Lucidum}

The stratum granulosum, 3-5 layers superficial to the stratum spinosum, is named because of the grainy appearance of the KRTs [15], [28]. The granular nature is due to accumulation of keratin and keratohyalin which are bundles of profilaggrin [35]. Lamellar bodies also accumulate in this layer before cells release the lipid into the stratum corneum [35]. The cells in the granulosum eventually flatten, lose their nucleus, and differentially terminate before reaching the stratum corneum [15], [28].

The stratum lucidum is another layer directly above the granulosum, and only present in thicker skin [15], [28]. The extracellular matrix is filled with a clear protein called eleidin, derived from keratohyalin [16].

\subsubsection{Stratum Spinosum}

Directly above the stratum basale is the stratum spinosum, a region of 8-10 layers of KRTs [28]. Cells are anchored by desmosome proteins and keratin [16]. The name of the layer comes from the desmosome 'spiny' appearance after histology staining [15], [28]. Spinosum KRTs also begin synthesis of keratin protein and lamellar bodies which are secretary lipid vesicles instrumental in maintaining the hydrophobicity of the skin [15], [33]. Interspersed within 
the KRTs are Langerhans cells, dendric antigen presenting cells that play an integral part in the epidermal immune response [36].

\subsubsection{Stratum Basale}

The stratum basale, also described as the stratum germinativum, is the deepest layer of the epidermis [15], [28]. It is a single layer of basal cells, cuboidal-shaped stem cells and precursors of the epidermal KRT cell lineage [15], [28]. Only 15\% of basal cells constantly undergo mitosis to rapidly produce KRTs that are eventually pushed upward to replace the previous superficial layer [15], [16]. The remaining basal cells are activated to undergo differentiation during wound healing [15], [16].

Merkel and melanocytes are non-keratinizing cells scattered among the basal cell layer [31]. Merkel cells receive and transmit touch sensations to the brain while melanocytes produce melanosomes and melanin pigment that protect cells from UV radiation [15], [16]. Melanin is upregulated after UV exposure and aids in photoprotection [30]. KRTs and melanocytes have a collaborative relationship; KRT paracrine factors regulate melanocyte function [31].

\subsubsection{Functions of the Epidermis}

The epidermis provides innate immunity with a physical barrier and antimicrobial secretion [15], [28]. From a lipid standpoint, the stratum corneum also maintains water secretion and prevents water loss from the body due to its high lipid content [16]. The transfer of water from the stratum corneum is measured by the Transepidermal Water Loss (TEWL) [16]. The high lipid content in the stratum corneum reduces TEWL, and perturbations of lipid content demonstrate how TEWL is affected. For example, mouse skin damaged by UV light showed an increase in TEWL [37]. In addition, severely burned skin leads to massive dehydration because of increased TEWL [15]. Furthermore, Gaucher and Neimann Pick disease are two disorders of sphingolipid enzymes that result in dysregulated skin permeation [29]. 


\subsection{Non-Melanoma Skin Cancer}

Cancer is described as the uncontrollable growth of abnormal cells [38]. Genetically mutated cells proliferate and form tumors with genomic and chromosomal instability which can lead to a metastatic cancer [38]. One cause of genetic mutations is UV radiation which enables mutated cells to ignore normal signaling processes that regulate proliferation and cell death [5]. Skin cancer manifests into two main types: Non-Melanoma and Melanoma.

Non-Melanoma Skin Cancer, abbreviated NMSC, is the most commonly diagnosed disease and is a significant health concern in terms of incidence rate and economic health care costs [1], [2]. According to the American Cancer Society, approximately 3.3 million Americans are diagnosed with NMSC each year [5]. NMSC prevalence has increased by 300\% since 1994 and generates over $\$ 650$ million in US healthcare costs [2], [39].

NMSC takes two main forms - Basal Cell Carcinoma (BCC) and Squamous Cell Carcinoma (SCC). BCC occurs most frequently in the human population, representing approximately $80 \%$ of all NMSCs [2], [5]. BCC and SCC originate in the basal and squamous cell layers respectively. In general, BCC rarely yields malignant tumors, while SCCs are more likely to invade and metastasize [5].

\subsubsection{Basal and Squamous Cell Carcinoma}

BCC, the most common skin cancer subtype, develops when mutated cells in the basal layer, the lowest region of the epidermis, become mutated and proliferate uncontrollably [5]. Over 3 million Americans are diagnosed with BCC each year [5]. Although rarely metastatic and easily treatable in early stages, surgical removal of BCC can leave scars, and identifying cancer phenotypes is crucial before a large lesion develops [5]. According to the Skin Cancer Foundation, BCC manifests into five common sore variants (Figure 3): open sore, red irritated skin, shiny (pearly) nodule, pink growth with elevated border, and wavy scar-like patch [5]. 

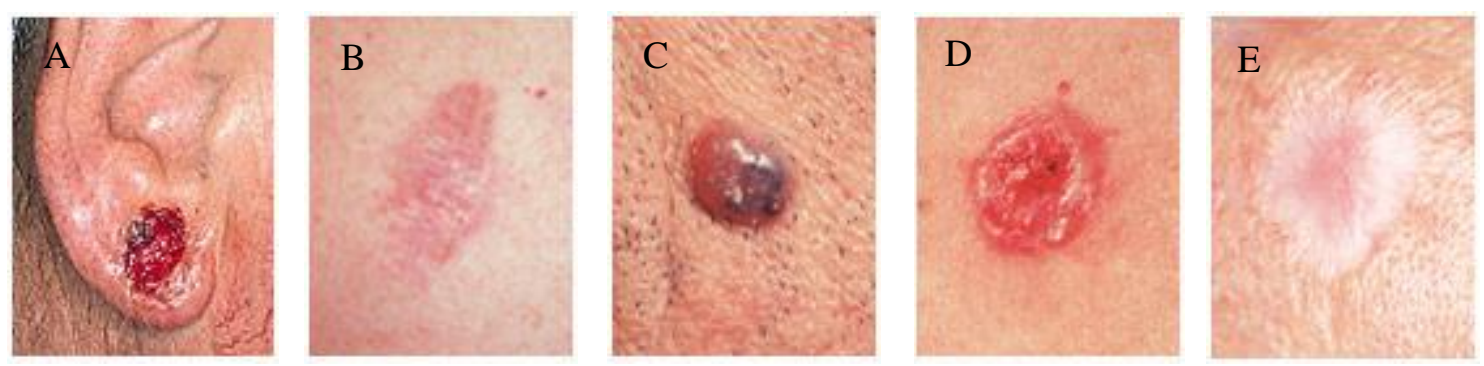

Figure 3: Types of BCC Tumor Skin Growths [5]. (A) Open Sore. (B) Red Patch of Irritated Skin. (C) Shiny Bump or Nodule. (D) Pink Growth. (E) Scar-Like Area.

SCC is the second prevalent NMSC subtype and involves cancerous proliferation of the squamous cells of the epidermis [5]. Over 1 million cases of SCC are diagnosed in the United States each year, and approximately 8,800 Americans die from the disease [5]. Furthermore, the incidence of SCC has risen 200 percent in America over the past 30 years. [5].

A small proportion of SCC can metastasize deeper into the dermis, or even spread to other organs, yielding significant surgical removal and a life-threatening prognosis [5]. SCC “precancers" like actinic keratoses, actinic chelitis, leukoplakia, and Bowen's Disease, are often benign and early stages of SCC [5]. The warning signs of SCC (Figure 4) include a crusty scaly patch, a bleeding growth, persistent bleeding open sore, and wart-like growth [5]. Early identification of precancers and abnormal lesions is essential for diagnosing and treating early stage cancer.
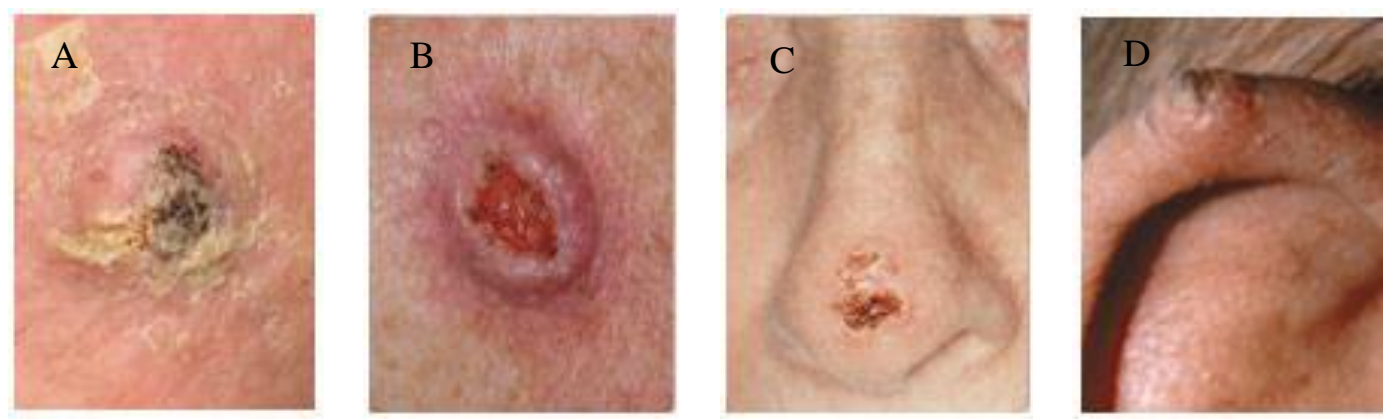

Figure 4: Types of SCC Tumor Skin Growths [5]. (A) Scaly red patch. (B) Elevated growth. (C) Persistent open sore that bleeds. (D) Wart-like growth. 
The main cause of skin cancer is prolonged exposure to sun and most cancers occur in skin areas exposed to sun such as the face, back, neck, shoulders, and ears [5]. However, skin cancer can develop from artificial sources of light like tanning beds. Certain subpopulations such as the elderly, fair-skinned, and the immunocompromised are at increased risk of developing BCC [5].

The treatments for BCC and SCC are commonly an outpatient procedure like Mohs Micrograph surgery, excisional surgery, cryosurgery, photodynamic therapy, and topic medication [5]. Often a biopsy is needed to make an accurate diagnosis [5].

\subsubsection{Melanoma and Merkel Cell Carcinoma}

Although less common in the American population, Melanoma and Merkel Cell Carcinoma (MCC) are invasive, aggressive, and metastatic forms of cancer [5]. Melanoma involves mutations in the melanocytes of the basal layer, while MCC begins in the Merkel cells connected to the nerves responsible for the touch sensation [5]. MCC is 40 times rarer than melanoma, and three times as deadly, killing one in three patients diagnosed with the disease [5]. Although Melanoma and Merkel Cell Carcinomas are more fatal than NMSC, this thesis focuses on NMSC because it is more prevalent in the human population.

\subsection{UV Light}

Ultra Violet Radiation (UVR) is a detrimental exogenous agent responsible for genome damage in all species [4]. In general, UV light is composed of three separate types differentiated by their respective wavelengths: UVA (320-400 nm), UVB (280-320 nm), and UVC (200-280 nm) [2]. UVA lies closest to the visible light spectrum (700-400nm) on a scale of increasing wavelength (Figure 5). Differing wavelengths yield differential penetration depths, with UVA penetrating deeper into the subcutaneous tissue while UVB only reaching the superficial epidermis/dermis layers [40]. 


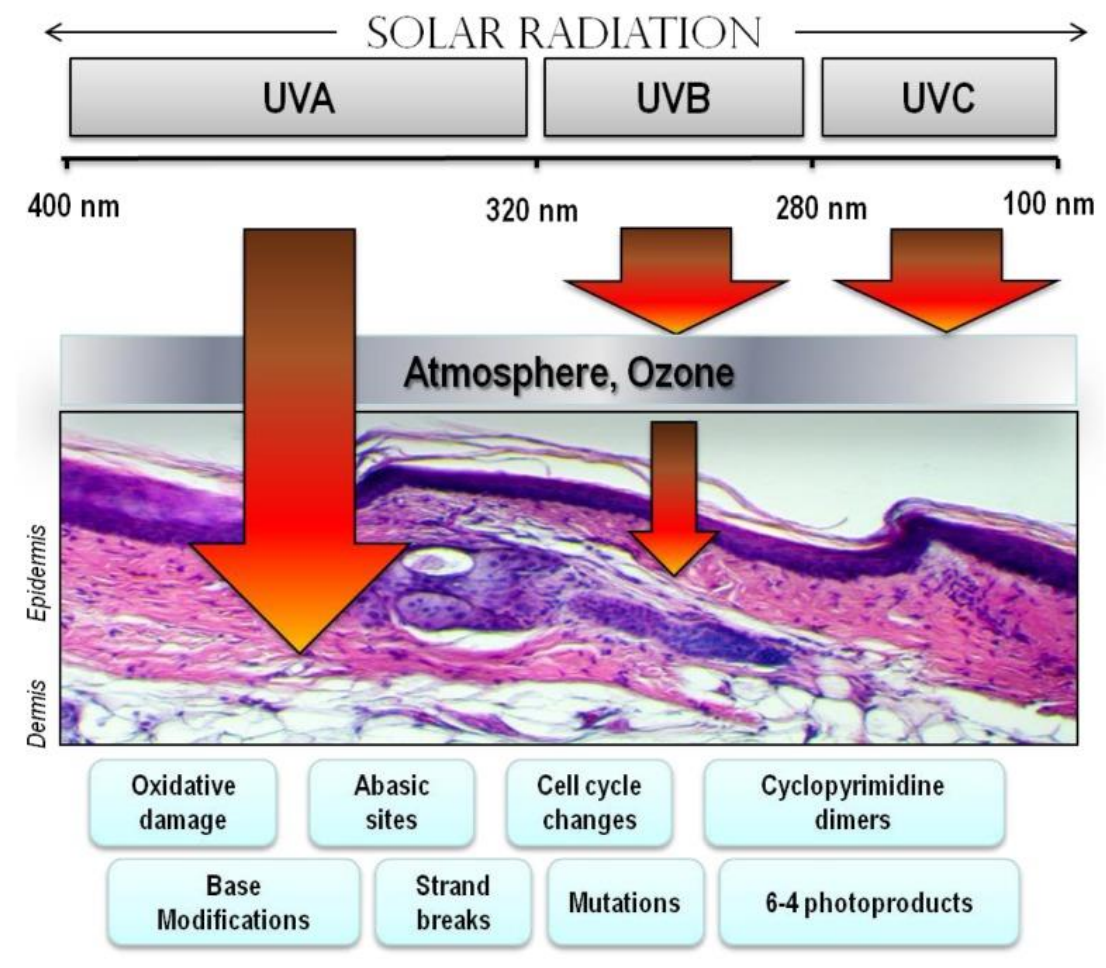

Figure 5: Differing UV Wavelengths (UVA, UVB, UVC) and Skin Penetration Depths [40]. UVA and UVB are wavelengths of primary concern for skin cancer. UVA radiation penetrates deeper into tissue while UVB is absorbed by the epidermis. UVB is responsible for DNA damage.

UVB radiation is the primary concern for skin damage. Comparatively, UVB, although only comprising $1 \%$ of the sun's total energy, is the most damaging of the three because UVA does not efficiently generate DNA damage and the ozone in the atmosphere effectively filters out UVC [3]. Thus, UVB is most relevant to skin cancer and most damaging. Most of the deleterious, cancer-inducing effects of UVB are caused by direct damage to DNA as described in the subsequent section [40]. Furthermore, UVR-genome damage incidence is increasing in the world because of ozone depletion [4]. Assessing the mechanism of damage and potential photoprotective agents is crucial to prevent the persistence of NMSC and other skin cancers.

\subsection{UV-Induced DNA Damage}

Deoxyribonucleic Acid (DNA) is the genetic instruction manual for a cell [15]. DNA is a molecule comprised of two interwoven complementary strands twisted into a ladder shape [15]. 
The backbone is made of alternating sugar and phosphate groups joined by four nitrogenous bases: Adenine (A), Thymine (T), Cytosine (C), and Guanine (G) [15]. Adenine and cytosine selectively hydrogen bonds with thymine and guanine respectively to form AT and CG base pairs [41]. Structurally, A, T, C, and G are heterocyclic aromatic compounds, meaning they are organic compounds with one or two carbon atoms replaced with a different atom (e.g. nitrogen) [41]. C and $\mathrm{T}$ are classified as pyrimidines (one ring nitrogen-carbon compound) and $\mathrm{A}$ and $\mathrm{G}$ are purines (two ring structures with pyrimidine fused to imidazole) [15], [41]. Together, the nitrogenous base and sugar backbone form a nucleotide, the building block of DNA [15]. The order of bases along the sugar-phosphate backbone determines the genetic code by affecting transcription and translation [15]. During normal DNA replication, DNA polymerase "proofreads" for errors in nucleotide insertion along the DNA molecule and mismatch repair enzymes further correct for deformities in DNA structure [41]. However, if an error in DNA nucleotides is not corrected, the mutations become permanent [41]. The cell no longer recognizes the DNA as mutated and uses the incorrect strand as a template for subsequent replication processes [41]. Eventually, the cell propagates the mutation which can yield a disease phenotype like cancer [41]. Error can occur either spontaneously or as a result of the environment (i.e. UV radiation).

UV-B radiation leads to the formation of lesions in DNA that alter the structure and form mutations [3], [4]. The main types of lesions include cyclobutene pyrimidine dimers (CPDs) and pyrimidine(6-4) pyrimidone photoproducts, also referenced as (6-4) photoproducts [3], [4].

\subsubsection{DNA Mutations}

When a skin cell is subjected to UV light, the DNA strands absorb a photon of high energy light which is then used to form extra covalent bonds between bases, forming a dimer or lesion [4], [18]. The two common mutations caused by UV are CPDs and (6-4) photoproducts; both involve adjacent pyrimidines (C or T) in the DNA molecule [4], [18]. Adjacent pyrimidines are weak spots for DNA mutation and are the likely site for UV damage [18]. CPDs form at a T-T 
site; the double bond between the $\mathrm{C}-4$ and $\mathrm{C}-5$ carbons breaks into a single bond and the energy is transferred to a bond between the C-4 carbons of the adjacent thymine [4], [18]. A ring structure connecting the two bases and mutating the DNA structure is ultimately formed [18]. On the other hand, (6-4) photoproducts occur between T-C adjacent bases and results when the C-6 carbon of the 5' side of the first base covalently binds to the C-4 carbon of the 3' adjacent pyrimidine (Figure 6) [4], [18].

A
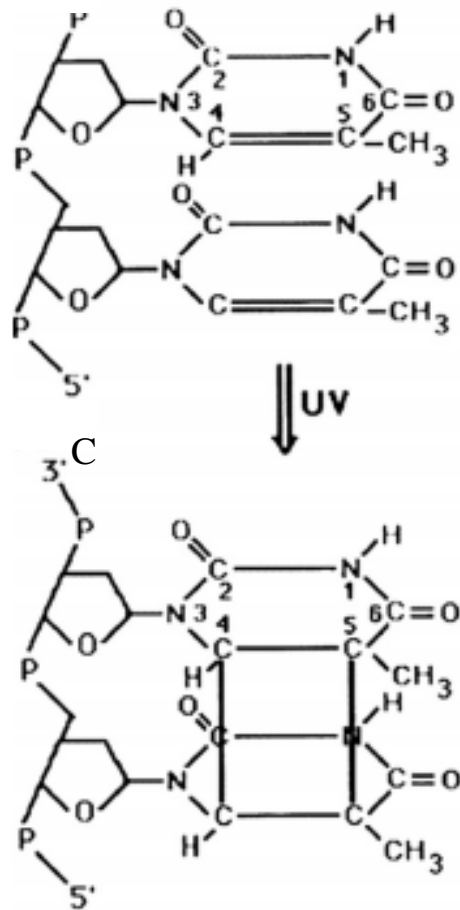
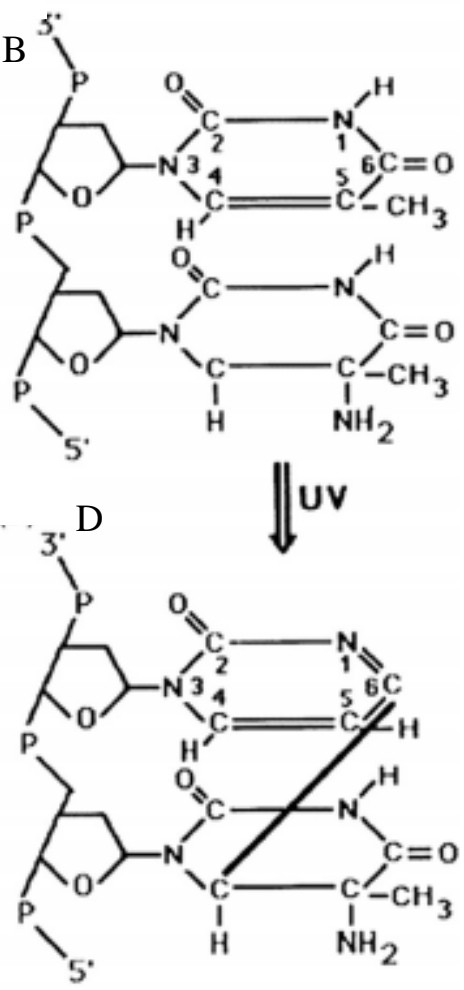

Figure 6: DNA Mutations because of UV [18]. (A) T-T adjacent bases. (B) T-C adjacent bases. (C) After UV, a cyclobutene pyrimidine dimers (CPD) forms. (D) After UV, a (6-4) photoproduct forms.

Both CPDs and (6-4) photoproducts greatly disturb and distort the DNA double helix, albeit (6-4) photoproducts to a greater degree [18]. Although CPDs are three times as likely to occur than (6-4) photoproducts and are more difficult to detect, the order of nucleotides, 
wavelength of light, and location of DNA can all affect the prevalence and nature of the DNA damage [18].

These mutations can lead to C-> T or CC-> TT nucleotide substitutions where a cytosine is replaced by a thymine during DNA replication, leading to incorrect incorporation of bases into the genetic code [18]. The CC-> TT substitution is so common in cancer cells that it is termed a 'UV signature mutation' [18], [19]. When this mutation occurs in oncogenes like the p53 tumor suppressor gene, the risk of developing skin cancer later increases [18].

UV radiation can also cause several other mutations like purine photoproducts, singlestrand breaks, and double-strand breaks [4], [18]. UVA is also known to create intracellular damage by the formation of Reactive Oxygen Species (ROS) like singlet oxygen and hydrogen peroxide [18]. Intracellular proteins and lipids can also absorb UV and generate damaging ROS [18].

Studies have also demonstrated that UVR can lead to local immunosuppression by the release of the cytokines TNF-alpha and IL-10, recruitment of macrophages, and decrease in Langerhans cells [19]. A reduced immune system increases the likelihood that a tumor cell will not be detected [19].

\subsection{DNA Damage Response Cascade}

More specifically, how does a cell detect DNA damage and initiate repair before a mutation is propagated? The answer lies in the DNA Damage Response (DDR), a host of cell pathways initiated by a damaging agent like UV radiation, ionization, or chemotherapy [6]. Of particular interest for this thesis is the effects of UVB radiation on DDR initiation and signaling (Figure 7).

The most important role of the DDR is to maintain the cell's DNA or genome [6]. When the DNA is damaged, molecular sensors on DNA like Mre11, NBS1, and Rad 50 detect the damage and signal to downstream proteins in the DDR cascade, ATM (Ataxia-Telangiectasia 
Mutated Protein) and ATR (ATM and RAD3-related) [6]. ATM and ATR are protein kinases that phosphorylate downstream protein targets in response to DNA strand breaks or lesions [6]. These protein targets include p53, CHK1/2, and p21 [6].

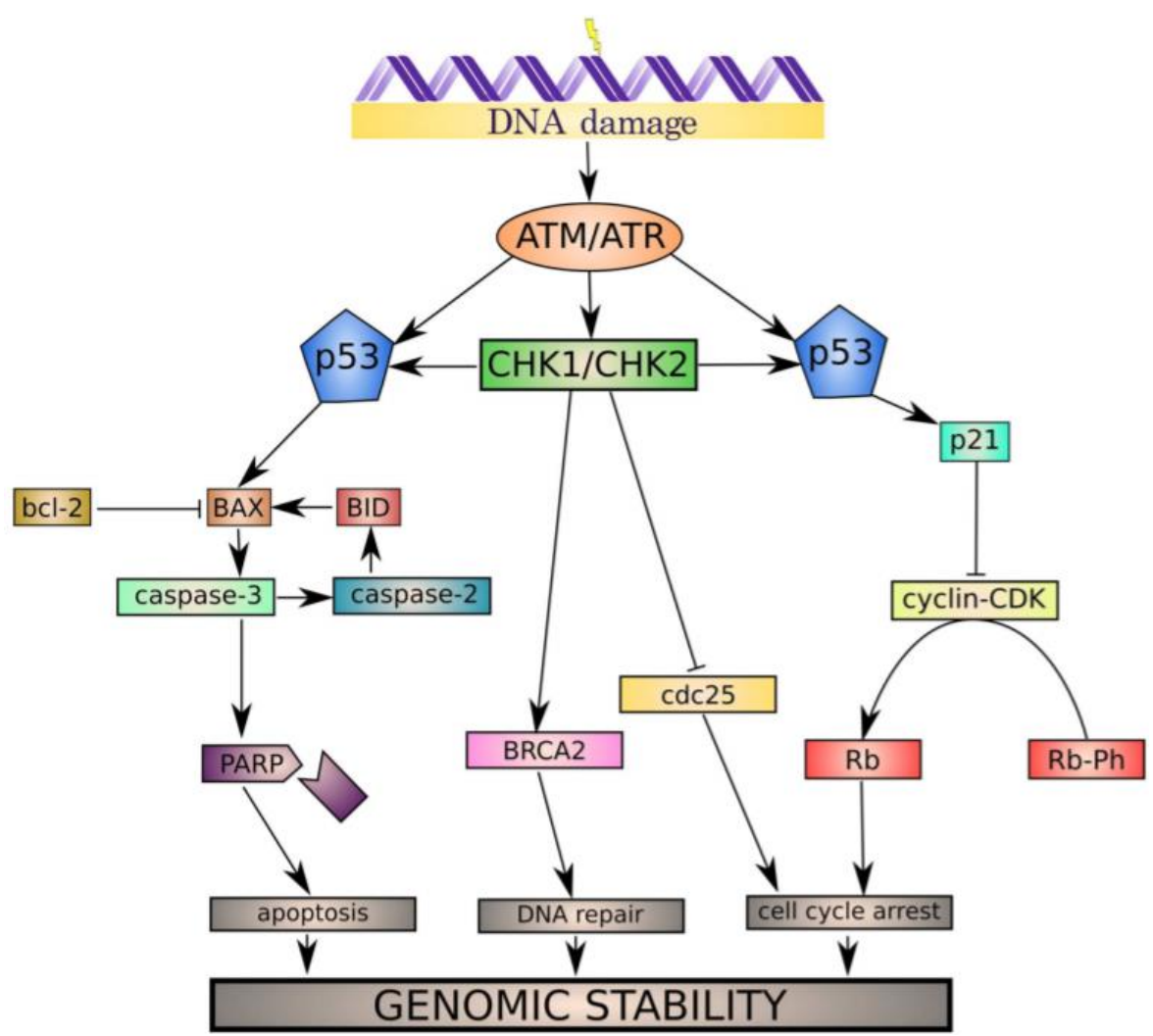

Figure 7: UV-Induced DNA Damage Response and Cell Pathways [6]. The goal is to maintain genomic DNA identity. DNA damage initiates ATM/ATR proteins which signal to p53 and CHK1/2 to either induce apoptosis, DNA repair, or cell cycle arrest.

In the DDR cascade, ATM/ATR eventually activates p53 and/or Checkpoint Kinase 1 and 2 (CHK1 and CHK2). The DDR ultimately results in three cell actions - apoptosis, DNA repair, and cell cycle arrest [6]. Apoptosis, DNA repair, and cell cycle arrest are all essential in maintaining genomic stability. All three will be explored in the following sections, with a particular emphasis on proteins p21 and p53 in apoptosis and cell cycle arrest signal cascades. 


\subsubsection{Apoptosis and p53 Tumor Suppressor Gene}

The tumor-suppressor protein $\mathrm{p} 53$ is an important component of the DDR apoptosis pathway [6]-[8]. Apoptosis, or programmed cell death, is a p53-dependent pathway and cancer defense mechanism for damaged cells [6]. Following ATM/ATR activation by DNA damage molecular sensors, p53 initiates BAX-mediated caspase-3 activation [6]. Caspase-3 cleaves PARP (poly-ADP ribose polymerase) which is a marker for cell apoptosis [6]. Thus, p53 protein plays an integral role in apoptosis.

Coined the "guardian of the genome", p53 functions as a transcription factor that turns genes on/off to induce cell cycle arrest and apoptosis [8]. The p53 protein is a tumor suppressor demonstrated to influence up to $50 \%$ of all cancers [7]. In physiologically normal cells, p53 is required for apoptosis of mutated cells before cell division. However, when p53 experiences a loss-of-function mutation, the protein loses its ability to effectively halt DNA replication of mutated cells [7]. Since the p53 protein is crucial in preventing tumorigenesis, a mutation in the gene codon can be deleterious. Signature mutation hot spots commonly occur at 5'-CCG and 5'TCG sequences in codons 196, 213, 245, 248, and 282 of the p53 gene in human keratinocytes [18].

The p53 protein is encoded by a gene located on chromosome 17 of humans [42]. However, the p53 protein needs to be activated and stabilized before it can directly activate or repress effector genes [8]. P53 can also indirectly regulate gene transcription by binding to transcriptional activators, repressors, and co-repressors [18]. Activation involves phosphorylation of the $\mathrm{p} 53$ protein at the amino terminus by ATM, ATR, CHK1, or CHK2 in response to DNA damage [8]. Phosphorylation prevents p53 binding with the MDM2 E3 ubiquitin-protein ligase which normally inactivates p53 when bound (Figure 8) [8]. As demonstrated in Figure 8, the tumor suppressor $\mathrm{p} 14^{\mathrm{ARF}}$ is also responsible for activation of $\mathrm{p} 53$ by degrading $\mathrm{p} 53-\mathrm{MDM} 2$ binding [43]. 


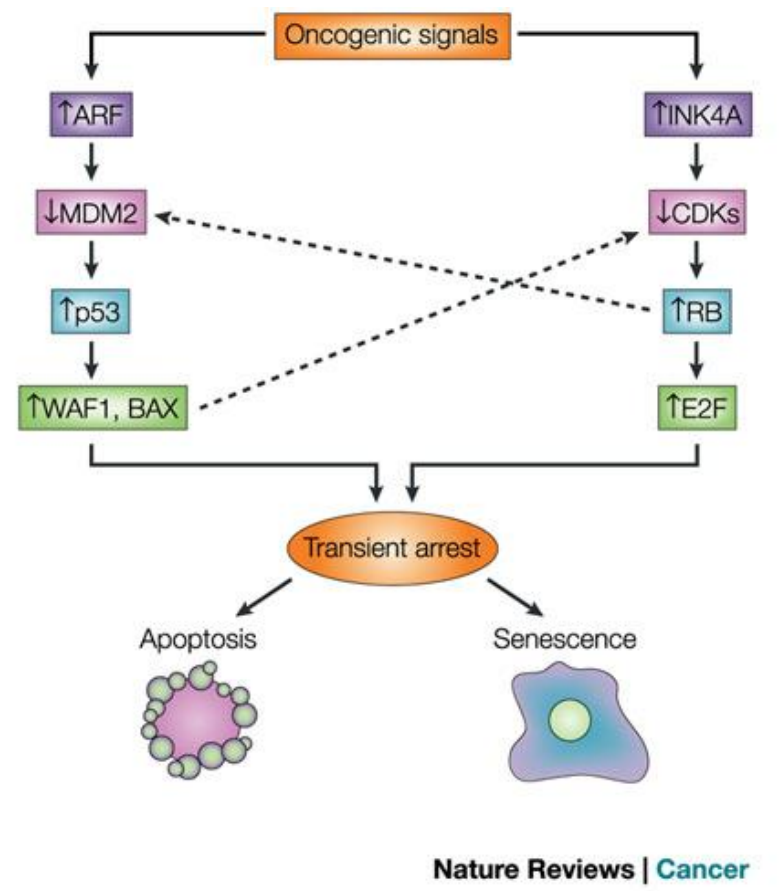

Figure 8: p53 Regulation Leading to Apoptosis and Cellular Senescence [43]. P53 increases WAF1 and BAX leading to either apoptosis or cellular senescence.

Once activated, p53 influences gene expression by binding to specific effector genes [6], [8], [43]. Via different pathways, p53 activation leads to both cell cycle arrest and apoptosis [6]. In terms of apoptosis, $\mathrm{p} 53$ functions both in the nucleus and cytoplasm via transcriptiondependent and independent methods respectively [8]. The main effector gene is PUMA, p53-upregulated modulator of apoptosis [8]. In the nucleus, p53 protein also activates BAX, which is a member of the Bcl-2 genes that regulate apoptosis [8]. P53 additionally moves out of the nucleus to the mitochondria, where it causes mitochondrial outer membrane permeabilization (MOMP); the mitochondria then releases pro-apoptotic cytokines [8]

From a cell cycle arrest standpoint, p53 activates p21 (Waf1 or Cip1) which is a cyclindependent kinase inhibitor that arrests DNA replication in the G1/S checkpoint [9]. P53 can also induce irreversible cell cycle arrest or senescence through a variety of different proteins [9]. P21 is described further in the following section. It is also important to note that p53 function is a 
highly context dependent and complicated pathway that varies based on the cell type, genome, microenvironment, and type of cellular stress [8].

\subsubsection{DNA Repair}

DNA repair, in contrast to apoptosis, is a p53-independent pathway [6]. Independent of p53, Checkpoint Kinases (CHK1/CHK2) activate BRCA2 which initiates internal cellular repair mechanisms [6]. For humans (and other eukaryotes), the process to resolve bulky mutations like CPDs and (6-4) photoproducts in DNA is predominantly via Nucleotide Excision Repair (NER) [18]. NER occurs in several steps [18]. In general, the damage is recognized by DNA polymerase and binding proteins [18]. A transcription complex of proteins binds to the damaged DNA lesion and unwinds the strand with helicase [18]. The DNA single-strand is then removed by endonucleases [18]. Finally, the gap is ligated and polymerized [18].

\subsubsection{Cell Cycle Arrest and P21 Cyclin-CDK Inhibitor}

Lastly, cell cycle arrest is both p53-independent and p53-dependent (Figure 7), but the p53 dependent pathway is of interest to this thesis [6]. ATM/ATR can activate p53 which leads to activation of p21, a cyclin-CDK inhibitor [6]. P21, also designated WAF1 or Cip1, is a protein that induces cell cycle arrest in a p53-dependent manner [9]. Before delving into p21's regulation of cell cycle arrest, it is important to detail the role of the cell cycle in a physiologically normal cell.

The purpose of the cell cycle is to duplicate the genome of a cell into identical daughter cells during mitosis [44]. The cell cycle consists of S phase, M phase, and various gap phases (Figure 9) [44]. During the S or synthesis phase, cells duplicate their entire genome, which usually takes 10-12 hours on average [44]. In M phase, or mitosis, cells undergo division into two identical daughter cells [44]. During mitosis, the replicated sister chromatids align along the metaphase plate, separate to opposite poles of the cell, then pinch off during cytokinesis [44]. 


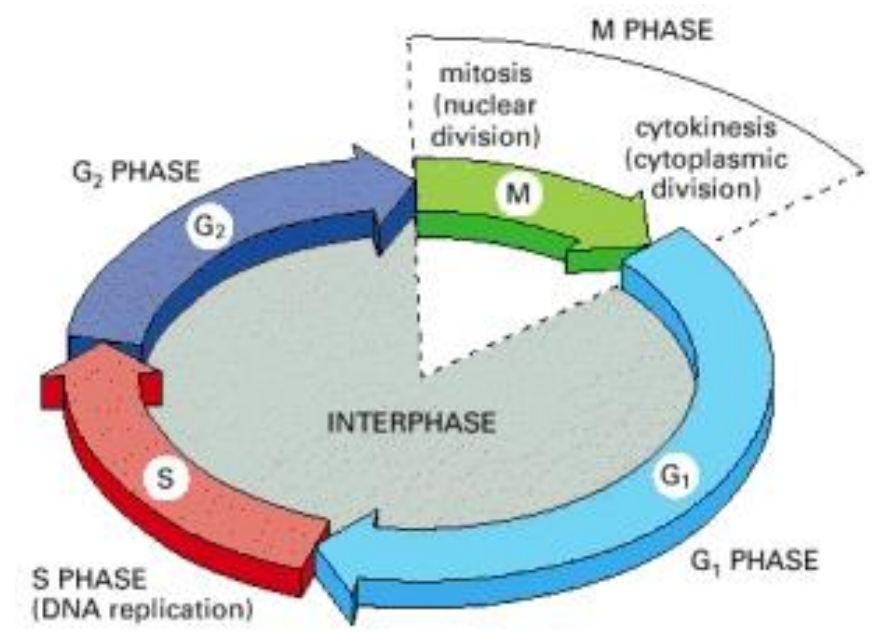

Figure 9: Normal Cell Cycle [44]. The cell cycle consists of G1, S, G2, and Mitosis with various checkpoints.

Separating synthesis and mitosis are $\mathrm{G}_{1}$ and $\mathrm{G}_{2}$ gap phases, which delay replication and division to allow for cell growth [44]. During $\mathrm{G}_{1}$ and $\mathrm{G}_{2}$, cells also monitor their environment and assess whether conditions are suitable for division [44]. The cues of intracellular proteins like p21 dictate whether the cell undergoes cell cycle arrest [44]. Within each phase is a series of checkpoints which allow the cell to stop, check for problems in the DNA, and halt the process for repair [44]. Cyclin-dependent Kinases (CDKs) regulate checkpoints by the binding of cyclins to the CDK to form a functional cyclin-CDK complex [38]. CDK2, CDK4, and CDK6 are important in interphase, while CDK1 is essential for mitosis [38].

The p21 protein plays a critical role in CDKs and cell cycle arrest. P21 is a small 165 amino acid protein that binds to CDK1 and CDK2 via a Cy1 motif in the $\mathrm{N}$-terminal and $\mathrm{Cy} 2$ in the C-terminal of the protein [9]. Binding disrupts the interaction between CDK and its cyclin substrates, therefore halting the cell cycle [9]. Recent studies demonstrate that a higher expression of p21 is associated with inactivation of genes necessary for cell cycle progression [9]. 


\subsection{Carcinogenesis}

Skin carcinogenesis initiates when a CPD or (6-4) photoproduct lesion evades the DNA repair process [18], [19]. The mutation is replicated, leading to a UV 'signature mutation' in a gene [18], [19]. When the mutation occurs in an oncogene or tumor suppressor gene, a tumor is likely to develop [18], [19].

Oncogene and tumor suppressor gene mutations are deleterious because they control normal cell division, apoptosis, cell cycle repair, and DNA repair [18]-[20]. Oncogenes are generated when normal proto-oncogenes are mutated; since proto-oncogenes regulate cell proliferation, a mutated oncogene yields uncontrolled cell growth [18], [20]. Tumor suppressor genes, on the other hand, slow cell division and encourage apoptosis. When a tumor suppressor gene experiences a loss-of-function mutation, the cell evades DNA repair, slowed proliferation, and apoptosis. The proliferation and migration of mutated cells from a pre-malignant lesion to a primary tumor is often stepwise (Figure 10).

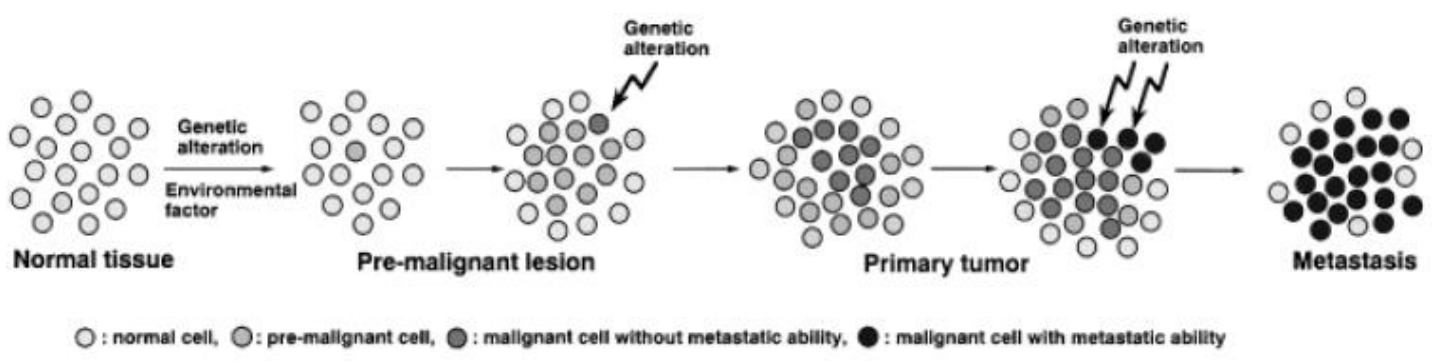

Figure 10: Etiology and Progression of Cancer [20]. Cancer results from repeated genetic alterations.

Normal tissue is exposed to an environmental factor like UVB radiation and some cells mutate. However, in order to progress from a pre-malignant lesion to tumor and metastasis, repeated instances of genetic alterations need to occur [20]. NMSC often develops after a lifetime of skin exposure and repeated sunburns. 


\subsection{Current Prevention of UVR induced DDR}

The main methods to circumvent UV damage in keratinocytes is prevention, predominantly by protective actions [5], [12]. These protective actions include avoiding sun during peak hours of the day, donning protective clothes like a hat and sunglasses, and applying a broad spectrum and high Sun Protection Factor (SPF) sunscreen [12]. Sunscreen is a crucial aspect of the photoprotection regime [5], [12]. In a 2016 poll of US dermatologists, $97 \%$ believed that the use of sunscreen lowers the risk of skin cancer, but $99 \%$ asserted that patients generally do not apply enough sunscreen during outdoor activities [21]. According to the Skin Cancer Foundation, any person over the age of 6 months should apply sunscreen daily, even if indoors or the day is cloudy [5].

Sunscreens are characterized by their Sun Protection Factor (SPF) and spectrum [5]. SPF is a quantitative gauge for the UVB protective ability. SPF 15, 30, and 50 filter 93\%, 97\%, and 98\% of UVB rays respectively [5]. A broad-spectrum sunscreen contains chemical and physical filters to protect skin from both UVA and UVB radiation. The Skin Cancer Foundation recommends applying about $1 \mathrm{oz}$ (a full shot glass) of sunscreen to receive the advertised SPF and spectrum benefits; however, most users only apply half the recommended dose to their skin [5]. Furthermore, sunscreen should be applied 30 minutes before sun exposure, and reapplied every 2 hours and following water contact or sweating because they lose efficacy over time [5]. Most Americans do not use sunscreen as recommended.

\subsubsection{Sunscreen Active Ingredients and Limitations}

Sunscreen protects skin via chemical and physical means which decrease UVA and UVB penetration into the epidermis and dermis [5]. Chemical ingredients (e.g. oxybenzone, avobenzone, octisalate, octocrylene) absorb UV radiation, while physical ingredients (e.g. zinc 
oxide, titanium dioxide) deflect UV rays [5]. A conventional sunscreen combines both chemical and physical filters.

Although the Food and Drug Administration (FDA) has currently approved over 15 sunscreen ingredients, some chemicals have received conflicting reports as to their safety and efficacy [10]-[12]. One such ingredient is the organic chemical filter oxybenzone (benzophenone-3). Oxybenzone provides broad spectrum protection by absorbing and quickly dissipating the UV energy by breaking chemical bonds [45].

Studies have demonstrated that oxybenzone causes allergic skin reactions, disrupts endocrine function, and pollutes the environment [10], [12]. In a 10-year study between 2001 and $2010,70.2 \%$ of approximately 24,000 patients exhibited dermatologic allergies to oxybenzone when patch tested [12]. In addition, a 2001 study conducted by Schlumpf et al. asserted that 21day-old rats fed doses of oxybenzone in their feed experienced hormone disruption [10], [12]. However, the dosage fed to the animal subjects were not physiologically relevant to the human. For example, it would take 277 years to reach the systemic concentration of oxybenzone in the rat study for a human applying a $6 \%$ oxybenzone FDA approved sunscreen at $2 \mathrm{mg} / \mathrm{cm}^{2}$ concentration [11]. Although different studies dispute the degree to which oxybenzone is cytotoxic in humans, given the prevalence of skin cancer, the need for more natural alternatives is important.

\subsection{Sphingomyelin as a Natural Alternative to Sunscreen}

Sphingomyelin (SM), a natural phospholipid, has demonstrated potential as a chemoprotective agent. Dietary administration of bovine milk sphingomyelin in mice reduced the formation and number of colon tumors [22]. Orally administered bovine milk SM was found to incorporate into the stratum corneum [23]. Additionally, the dietary SM that localized in the skin reduced Transepidermal Water Loss (TEWL) and increased the water content of skin after UVB 
radiation [37]. Lab research on KRT monolayers by De Guzman and Campbell et al. have also demonstrated promising results for SM photoprotection [13], [14]. Before exploring past research, a general overview of lipids, SM, and metabolite roles in the DDR is necessary.

\subsection{Sphingolipids}

In general, lipids are organic compounds insoluble in water but soluble in organic solvents [46]. According to LIPID Metabolites and Pathways Strategy (LIPID MAPS®) database, there are eight main lipid classifications: fatty acyls, glycerolipids, slycerophospholipids, sterol lipids, saccharolipids, prenol lipids, polyketides, and sphingolipids [46]. Because of the complex structural nature of lipids, classification can be subjective, and the LIPID MAPS database categorizes lipids based on their chemically functional backbone in an effort to make organization more universal [46]. Two lipids that play integral roles in cell signaling and the DNA damage response are sphingomyelin and ceramide, both classified under the sphingolipids branch and often described as bioactive because they modulate cell signaling and function [46]. Sphingolipids are important cellular components, representing 10-20\% of all cellular lipids [47]. Figure 11 depicts the structure of sphingomyelin and its common metabolites: ceramide, sphingosine, and sphingosine-1-phosphate [48]. More information regarding the structure and function is detailed in subsequent sections. 


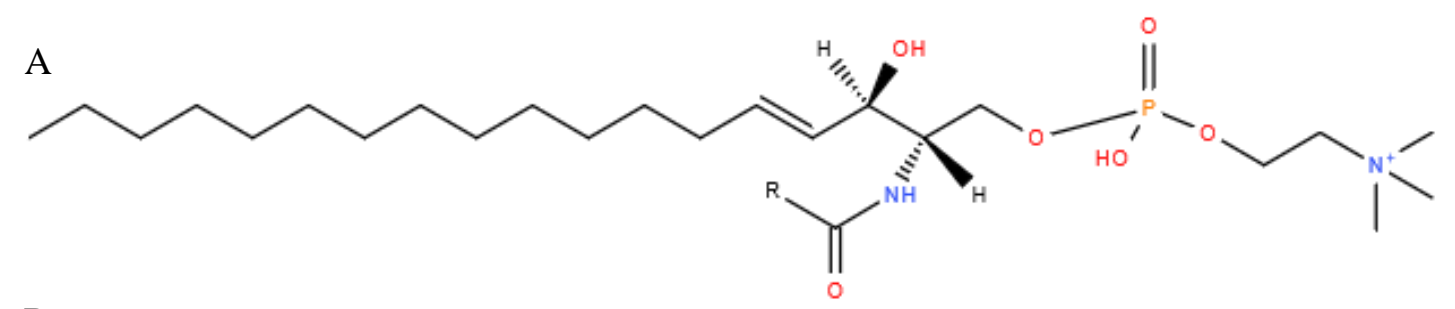

$\mathrm{B}$

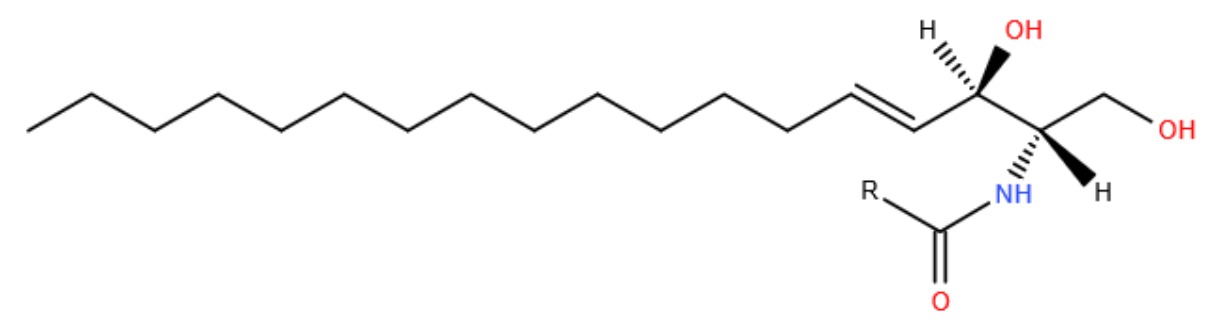

$\mathrm{C}$
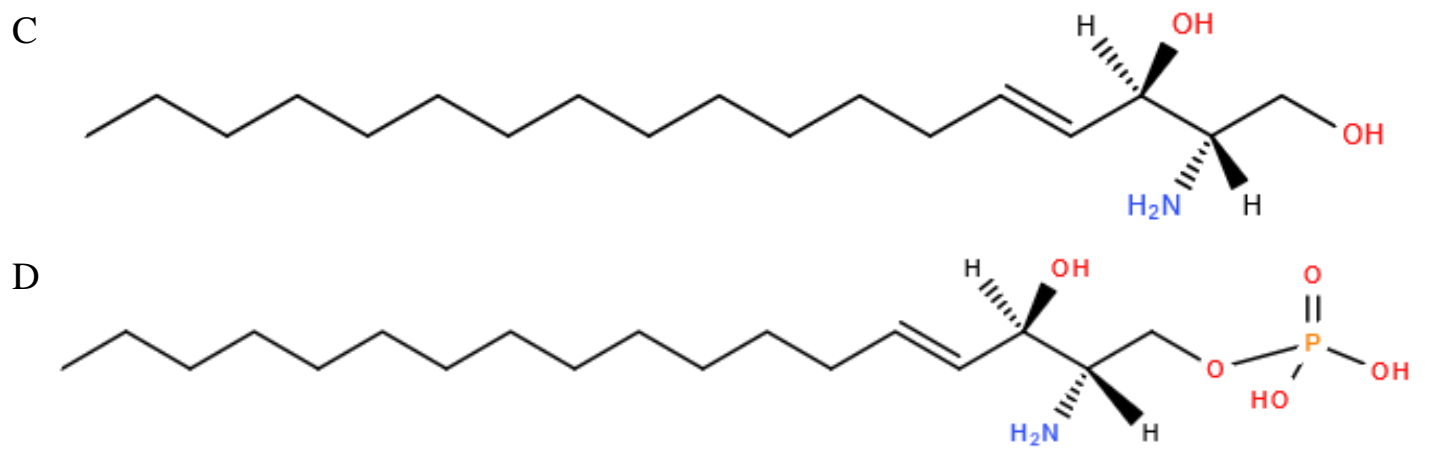

Figure 11: General Chemical Structures for Sphingomyelin, Ceramide, Sphingosine, and Sphingosine-1-Phosphate [48]. (A) Sphingomyelin, (B) Ceramide, (C) Sphingosine, (D) Sphingosine-1-Phosphate (S1P).

Sphingolipids regulate numerous biological functions and properties. For example, SM has been shown to influence the properties of the plasma membrane (fluidity, thickness and curvature) by closely associating with cholesterol and other sterols in lipid raft microdomains [47]. Subsequent cell signaling and protein sorting are influenced by membrane composition [47]. As previously described, SM also affects skin Transepidermal Water Loss (TEWL) [16]. Finally, $\mathrm{SM}$ is a precursor of bioactive lipid metabolites including ceramide and sphingosine-1-phosphate which mediate apoptosis, p21, and p53 levels after UV damage through cellular targets [6], [47]. Cells sense perturbations in lipid levels by soluble and transmembrane proteins that modulate sphingolipid metabolism [47]. Imaginably, maintaining lipid homeostasis is of utmost importance for the cell in order to ensure proper cell function and phenotype [49]. For example, Neiman 
Picks, Tay-Sachs, Gaucher's, and Farber's disease result from deficient lipid homeostasis mechanisms [47], [49], [50]. The following sections highlight sphingomyelin and ceramide, with a particular emphasis on ceramide due to its implications in UV damage, apoptosis, p21, and p53 [6], [47].

\subsection{Sphingomyelin and Ceramide Metabolism}

Sphingomyelin (SM) is a complex phospholipid composed of a phosphorylcholine head and two nonpolar hydrocarbon chains of varying lengths (Figure 11A) [48]. SM is naturally present, in low levels, on the outer leaflet of the plasma membrane associated with cholesterol in lipid raft domains [49], [51]. SM is also generated intracellularly by the modification of ceramide by SM synthase in the Golgi body, then it is trafficked to the plasma membrane [27]. SM degradation is also a fundamental homeostasis mechanism. In the plasma membrane and endosomes/lysosomes, aSMase and nSMase cleave sphingomyelin into ceramide [6], [27]. Ceramide then influences downstream cellular processes [6].

Ceramide is the hydrophobic backbone of complex sphingolipids including sphingomyelin, cerebrosides, and gangliosides (Figure 11) [52]. The structure of ceramide is a variable chain length fatty acyl bound to a sphingosine backbone [52]. The fatty acid bonds are generally saturated or monosaturated. Ceramides with chain lengths C16-24 are most common in mammalian cells, however, C26-C36 are present in some epidermal keratinocytes undergoing differentiation [52]. In addition, ceramide structures differ depending on the location of a hydroxy $(-\mathrm{OH})$ group. Ceramides with an $-\mathrm{OH}$ functional group bound to the second carbon $(\mathrm{C} 2)$ in the variable chain length are termed alpha-hydroxy fatty acids, and ceramides with -OH bound to the last carbon are appropriately termed omega-hydroxy fatty acids [52]. Because of their chemical structure, ceramides are highly hydrophobic (water-repellent) and exhibit low polarity and insolubility in water [52]. 
Ceramide metabolism is responsible for cell signaling which in turn regulates a myriad of cell responses including proliferation, apoptosis (programmed cell death), and cell senescence [6]. Lipid metabolism is a multi-faceted process with numerous sphingolipids that orchestrate cellular processes [6]. The focus of this thesis will be on the ceramide-sphingomyelin link, and the enzymes, proteins, and cellular machinery that regulate degradation and catabolism of these bioactive lipids. The generation, metabolism, and even location of ceramide in a cell is important for mediating cellular response [6], [27], [53].

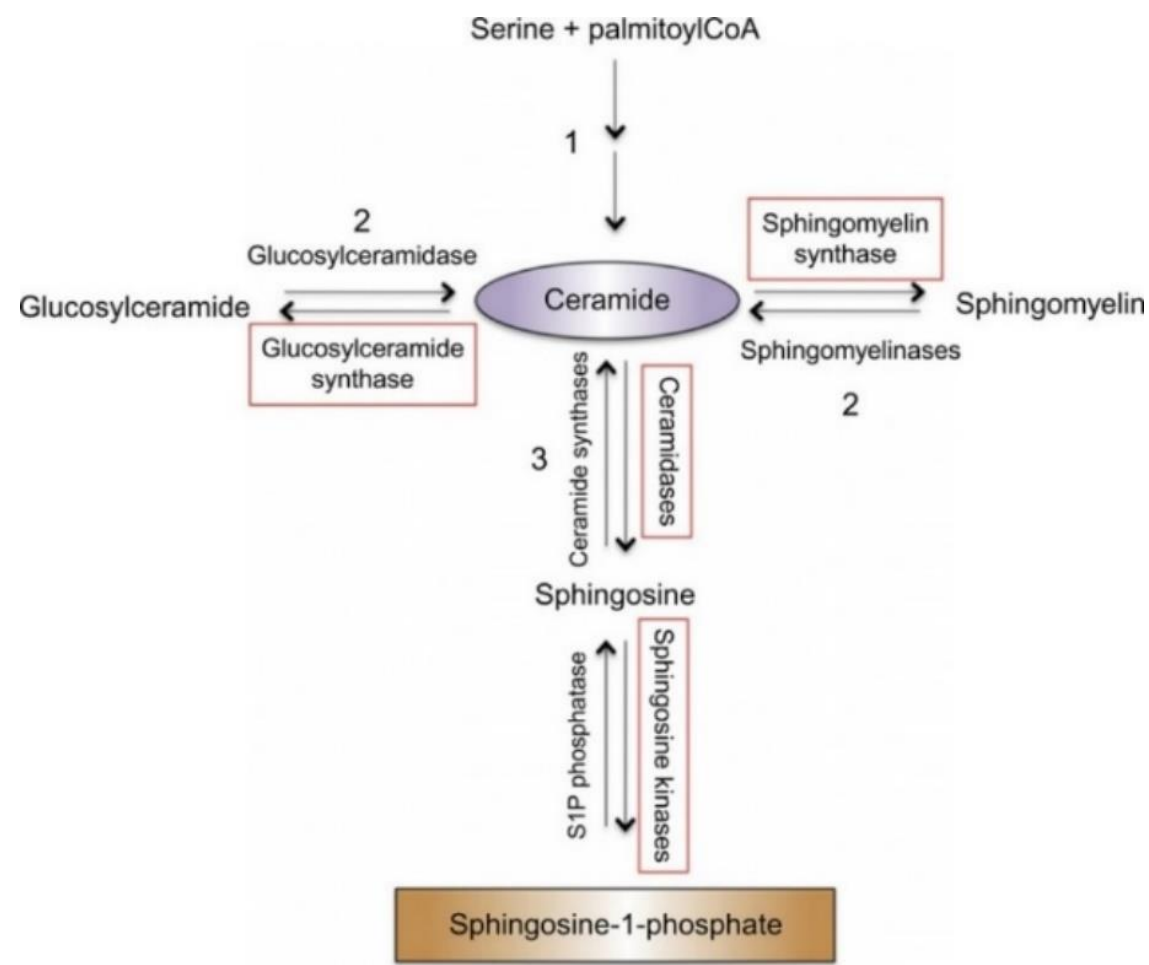

Figure 12: Pathways of Ceramide Generation and Metabolism [53]. Pathway 1 represents de novo synthesis, pathways labeled 2 are complex sphingolipid hydrolysis to ceramide, and pathway 3 is the salvage pathway.

In the study of ceramide, it is useful to separate metabolic activities into pathways that result in the modification of ceramide (either through metabolism into simpler products or as a building block for more complex sphingolipids) and pathways that form ceramide (Figure 12) [53]. Ceramide is generated through three independent pathways, each involving different enzymes (Figure 12) [53]. The first pathway is de novo synthesis. The de novo pathway occurs in 
the Endoplasmic Reticulum (ER) and starts with serine and palmitoyl CoA [6], [52], [53]. Serine and palmitoyl CoA are condensed to 3-keto-dihydrosphingosine by the enzyme serine palmitoyltransferase (SPT) [6], [52], [53]. Next, 3-keto-dihydrosphingosine is broken down to dihydrosphingosine (sphinganine) then further reduced to dihydroceramide by ceramide synthase (CERS) [6], [52], [53]. CERS is present in six different isoforms which have an affinity for different acyl CoA backbone lengths [6]. Finally, dihydroceramide is converted to ceramide by dihydroceramide desaturase (DEGS) [6], [52], [53]. After de novo ceramide formation in the ER, ceramide is trafficked to the Golgi by the Ceramide Transfer Protein (CERT) where it is further processed [52].

The second mechanism for ceramide generation involves hydrolysis of the complex sphingolipids SM and glucosylceramide [6], [52], [53]. Of interest for this thesis is the hydrolysis of SM to ceramide, which occurs in the plasma membrane and to some extent in the endosomes/lysosomes circulating in the cytosol of cells. The enzyme sphingomyelinase (SMase) degrades sphingomyelin to ceramide and a free phosphcholine head [6]. Three different SMase enzymes exist and vary based on the $\mathrm{pH}$ of the target location [6], [52], [53]. In the plasma membrane, both neutral SMase (nSMase) and acidic SMase (aSMase) are present to hydrolyze SM to ceramide [6]. In the endosomes/lysosomes of cells, aSMase is mainly responsible for the hydrolysis [6]. Lastly, alkaline SMase (alkSMase) is found in the digestive tract and remains the least characterized SM enzyme [6].

The third mechanism, the Salvage Pathway, involves the recycling of sphingosine from sphingosine-1-phosphate (S1P) by S1P Phosphatase in the lysosome and endosomes of cells [54]. S1P accumulates after sphingosine phosphorylation by Sphingosine Kinases and is a vital bioactive lipid implicated to cancer [6]. Figure 13 includes a summary representation of sphingolipid metabolites and relative locations in either the plasma membrane, Golgi, endoplasmic reticulum, and endosomes/lysosmes [27]. 
Ceramide degradation mainly occurs with acidic/neutral ceramidases which break down ceramide to sphingosine (Figure 13). Like ceramide generation via SMases, ceramide degradation by ceramidases occurs in the plasma membrane and endosomes/lysosomes of cells [6], [27]. The eventual byproducts of ceramide catabolism in the plasma membrane are sphingosine and S1P [6], [27]. In the Golgi of a cell, ceramide is modified by SM synthase to form sphingomyelin [6], [27]. Lastly, ceramide present in the ER can be degraded to sphingosine (and eventually S1P) by alkaline ceramidases [27].

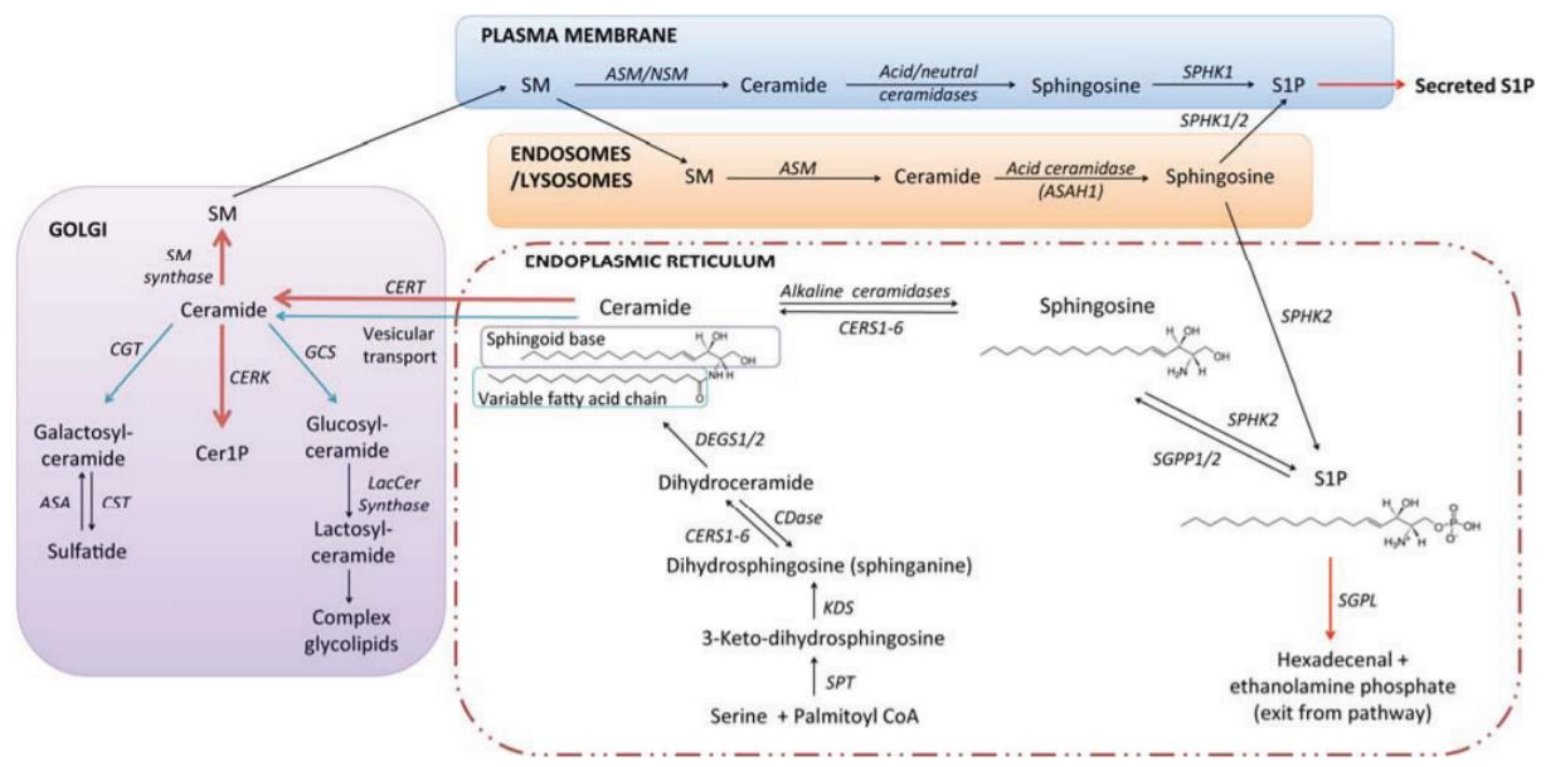

Figure 13: Summary of Sphingolipid Metabolism and Location in a Cell [27]. Ceramide is metabolized to sphingomyelin and S1P in different cellular locations.

\subsection{Sphingolipids, DDR Cascade, and Carcinogenesis}

Phospholipids metabolites can have either pro-survival or pro-apoptotic effects, and the regulation of enzymatic metabolism is key to determining which sphingolipids are produced, and their effect on UV-irradiated skin cells [6], [55]. Various bioactive metabolites are responsible for initiating the DDR including ceramide (Cer), sphingosine, and sphingosine 1-phosphate (S1P) [6]. Ceramide is one of the central hubs of the DDR and regulates cell death by inducing a proapoptotic cellular phenotype [6], [55]. 
Ceramide apoptotic mechanisms originate by the targeting of CAPPs (ceramide-activated Ser-Thr protein phosphatases), enzymes that remove phosphate groups from proteins [55]. One such CAPP is PP2A (protein phosphatase 2A) which inactivates the anti-apoptotic kinase AKT (serine/threonine-specific protein kinase) also known as protein kinase B and upregulates expression of p53 (Figure 14) [55]. Ceramide also activates PKC (Protein Kinase C)-zeta which downregulates AKT [55]. In addition, ceramide signals to ASK1 (Apoptosis Signal-regulating Kinase 1) and upregulates pro-apoptotic JNK, p38, and BAX levels [55]. Furthermore, prosurvival BCL-2 is downregulated [55]. These pathways eventually cause MOMP (Mitochondrial Outer Membrane Permeabilization) and release of the apoptotic protein cytochrome $\mathrm{C}$ and caspases [54]-[56]. Cathepsin D protein also enables apoptosis, in a mitochondria-independent manner [55].

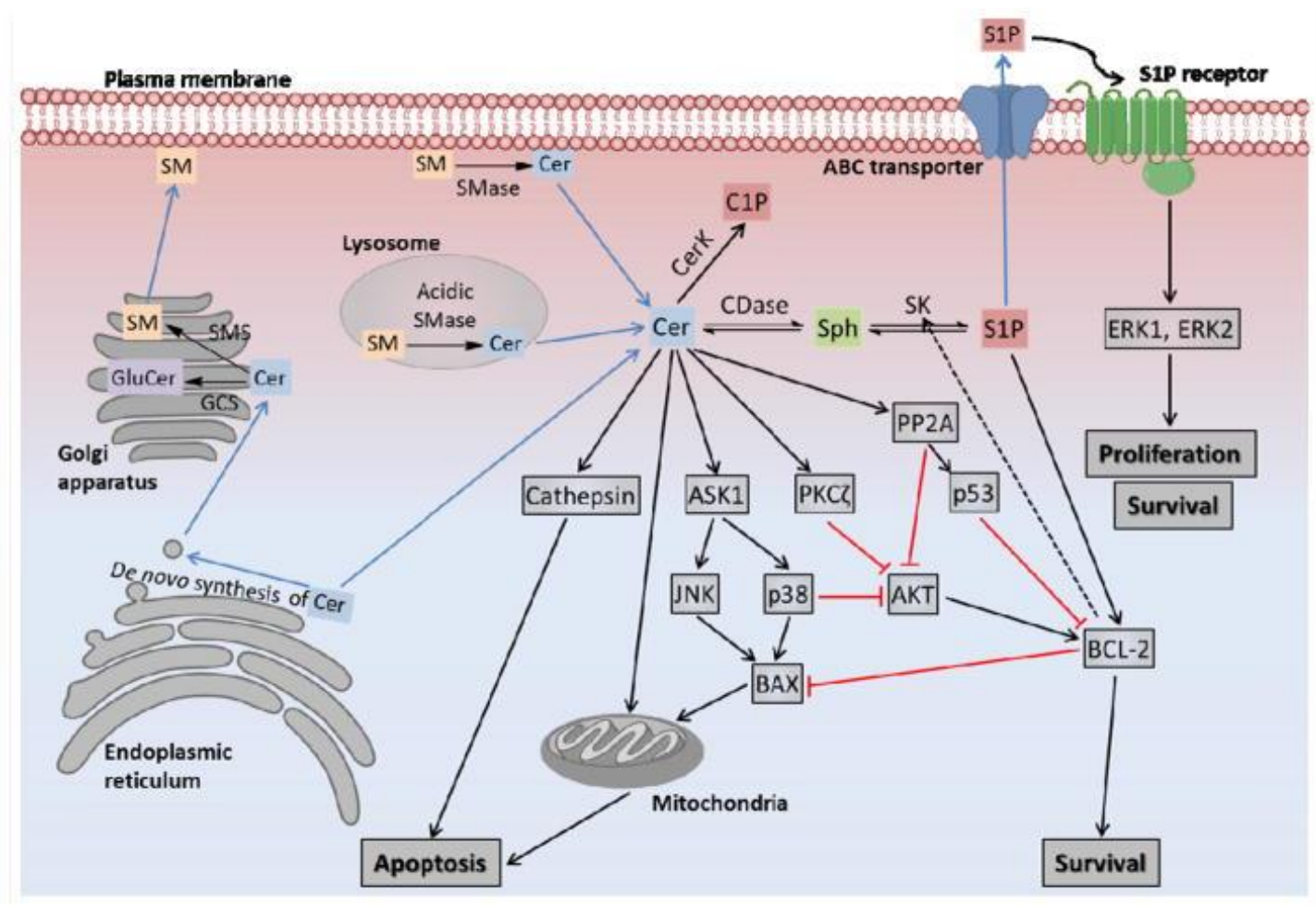

Figure 14: Ceramide Mechanisms for Cellular Apoptosis [55]. Ceramide and S1P mediate contradictory cell functions.

Figure 14 also demonstrates the importance of S1P, which has contradictory functions compared to ceramide [6], [55]. Ceramide, a pro-apoptotic lipid, and S1P, a pro-survival lipid, 
form a hypothetical 'rheostat' or 'scale' that can be tipped towards either apoptosis or cell survival depending on the type and amount of lipid available in a cell [6], [55]. Thus, the lipid metabolite produced in a cell greatly affects cell fate [6]. For example, in cancer cells, the prosurvival genes for S1P are upregulated, and in turn, the pro-apoptotic genes for ceramide production are downregulated [53]. In addition, accumulation of ceramide is linked to more cellular apoptosis as a protection mechanism for damaged cells [6], [53], [55]. Current anti-cancer treatments point to the apoptotic and tumor suppressive nature of ceramide [55]. Therefore, common treatments either introduce exogenous ceramide supplements to encourage cellular apoptosis, or inhibit ceramide metabolism [55]. The ceramide-S1P rheostat and complexity of lipid metabolism necessitate further research into mechanisms, homeostasis, and potential treatments for UV-induced DDR and cancer.

\subsection{Bovine Milk Sphingomyelin Past Research and Limitations}

Bovine milk SM has demonstrated significant potential as an anti-cancer treatment in the colons of mice [22]. In addition, oral consumption of SM in a mouse model demonstrated SM accumulation in the skin and a decreased skin TEWL after UVB [16], [37]. Previous work by De Guzman and Campbell from the Cal Poly Bioimaging Lab suggested bovine milk SM photoprotection for a monolayer of KRTs [13], [14]. P21 and p53 levels statistically decreased in SM-treated cells after UVB radiation compared to the control without SM [13], [14]. The results suggested photoprotective effects of bovine milk SM on KRT monolayers after UVB radiation.

However, these past studies were limited in that the mechanism of action for bovine milk SM photoprotection was not addressed. Direct visualization of intracellular FSM distribution by fluorescence for various conditions is necessary as a way to address this limitation. In addition, the cell model was a 2D monolayer, and moving to a third dimension could prove more physiologically relevant. 


\subsection{Probing In Vitro Lipid Dynamics with Fluorescence}

In order to validate the mechanisms occurring in a cell, it is important to study lipid dynamics in vitro. Imaging proves to be a valuable method for assessing lipid distribution in a cell and provides more spatial information than High Performance Thin Layer Chromatography (HPTLC) and associated methods [57]. However, tracking intracellular distribution of bovine milk SM is difficult because the phospholipid is not fluorescent under normal conditions.

Fluorescently tagged sphingomyelin has come to the forefront of lipid research, with multiple research groups developing novel methods to identify SM for intracellular trafficking studies and metabolism with imaging [58]-[60].

C6- NBD Sphingomyelin (abbreviated FSM in this thesis), manufactured by Avanti Polar Lipids, is one such example (Figure 15). The chemical structure differs from their bovine milk chemical by the addition of a fluorescent tag, indicated by the dashed box [61]. Despite the NBD attachment, FSM proves to be a sufficient analogue because its compact shape limits functional disturbance of in vivo processes [62].
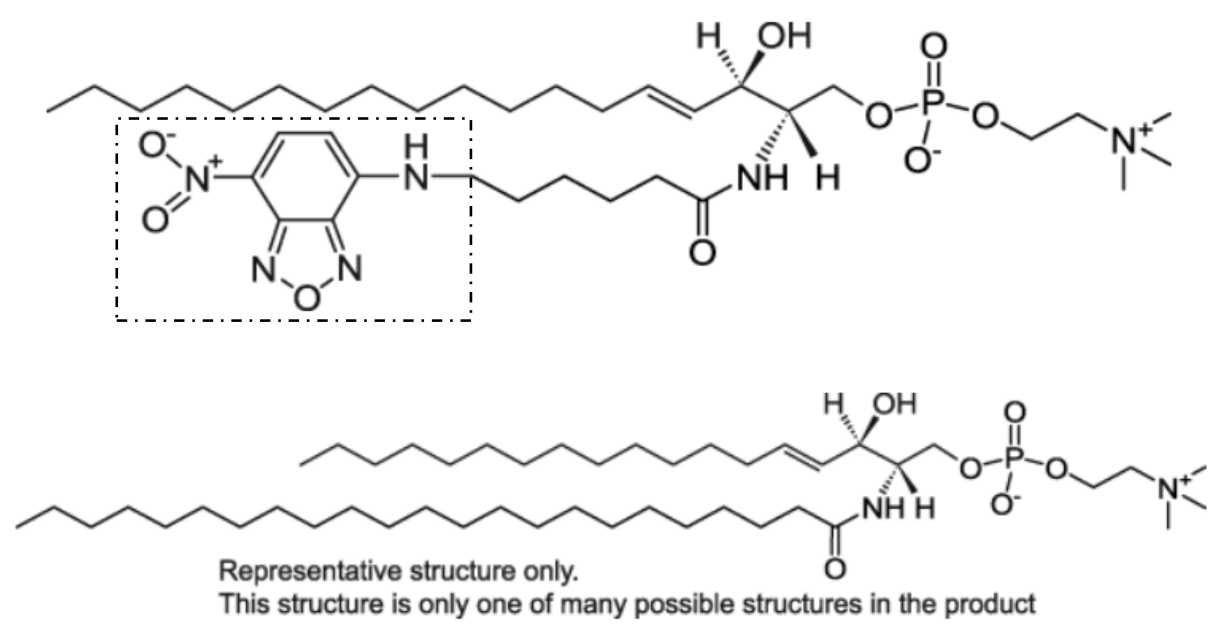

Figure 15: Avanti Polar Lipid Diagrams of Molecules [61]. 
Koval and Pagano et al. led first efforts to characterize FSM in Chinese Hamster Ovary (CHO) cells. Plasma membrane staining followed by rapid entry and Golgi accumulation was observed [60]. FSM was introduced (pulsed) to cells at $37^{\circ} \mathrm{C}$, back-exchanged with BSA on ice to remove residual FSM outside the cell plasma membrane, then chased with a $37^{\circ} \mathrm{C}$ media exchange to assess FSM trafficking [60]. Koval and Pagano's research was also repeated by other groups including Hao et al. and Kok et al. [58], [59]. FSM internalization was found to be temperature-dependent and trafficking rate declined at lower temperatures [58], [59]. Exogeneous administration of FSM was determined to be internalized by endocytosis into vesicles and recycled by three main mechanisms: 1) Rapid recycling from endosomes to the plasma membrane, 2) endosome to lysosomes for degradation to downstream metabolites, 3) endosomes to Golgi [63].

A novel probe called Lysenin has recently been developed for fluorescence detection of endogenous cellular SM [64]. Lysenin is a 41-kDa protein obtained from purified coelomic fluid of the earthworm Eisenia foetida [64]. This protein binds selectively to SM, as demonstrated by ELISA, TLC, and other methods [64]. Mound et al. used lysenin, in conjunction with FSM, to visualize lipid raft organization in the plasma membrane of KRTs [65]. Colocalization of lysenin with FSM signal was quantified, and Mound demonstrated that lysenin signal diminished after the addition of SMase, an enzyme that degrades SM [65]. The signal obtained was predominantly concentrated around the plasma membrane [65]. The heterogeneity of lysenin staining was also described and the overall signal declined as the cells reached senescence [65].

Although published research predominantly uses an exogenous version of ceramide or lysenin with a direct fluorescent tag, immunofluorescence assays have also been developed for both lysenin and ceramide [64], [66]. For endogenous SM staining, lysenin is first bound to SM, then an anti-lysenin primary antibody is introduced followed by secondary fluorescent tag [64]. Ceramide immunofluorescence also involves an antibody against ceramide and a secondary 
fluorescent tag [66]. Optimization of each step in the staining procedure was necessary to obtain a quality signal. Both lysenin and ceramide antibodies prove useful for colocalization experiments and probing lipid trafficking in a cell.

\subsection{Tissue Engineered 3D Skin Culture}

Tissue engineering is a field of regenerative medicine aimed at creating accurate in vitro models to mimic the in vivo characteristics of human tissue [67]. This field traditionally combines cells, a biomaterial scaffold, and growth factors or other chemical/external stimuli to guide cell proliferation into a structure that better recapitulates tissue [67]. From a tissue engineering standpoint, a 3D model proves potentially more advantageous over previously described KRT 2D monolayer studies because multiple layers of cells can be grown and studied [13], [14]. The many layers of stratified KRTs in vivo can be mimicked in vitro. Developing tissue engineered skin for assessing BSM and FSM photoprotection after UV is a scientifically advantageous goal to progress research in this field [13], [14].

Based on a 2017 review of current tissue engineered skin technologies, most in vitro skin models resemble a flat sheet, with keratinocytes and/or fibroblasts cultured on top a natural and/or synthetic biomaterial scaffold (Figure 16) [68]. For example, Apligraf ${ }^{\mathrm{TM}}$ combines keratinocytes with fibroblasts and collagen to recreate both the epidermis and dermis [68] 


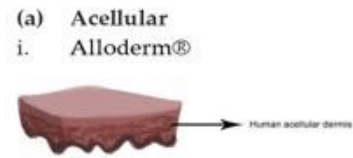

(b) Epidermal Autologous i. Cell Spray

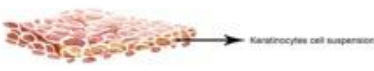

(c) Dermal Autologous i. Hyalograft 3D

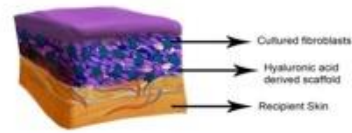

(e) Xenogenic Dermal i. Permacol

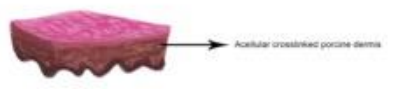

ii. Biobrane 18

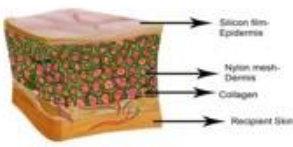

ii. Epicel

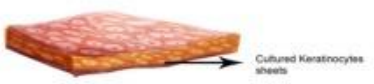

(d) Dermal Allogenic

i.TransCyte

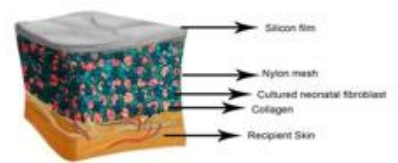

(f) Epidermal/ Dermal (Composite) Autologous

i. Tissue tech autograft system

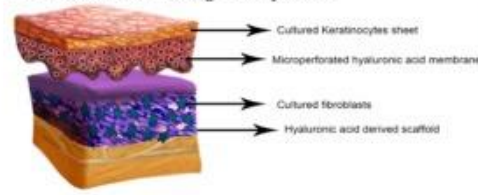

iii. Integra® DRT

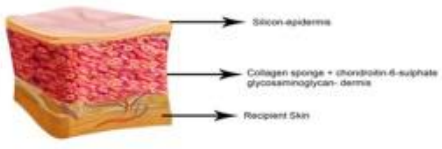

iii. Laserskin

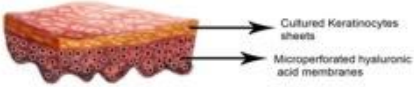

ii. Dermagraft

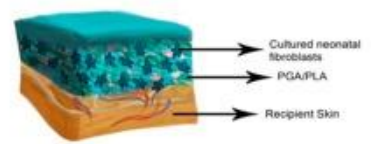

(g) Epidermal/ Dermal (Composite) Allograft i. Apligraf

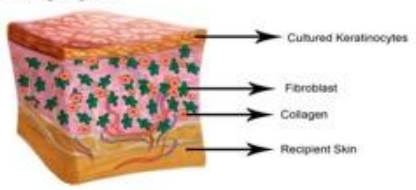

Figure 16: Review of Current Tissue Engineering Skin Products [68].

Despite the success of many sheet-based tissue engineering products for skin, some limitations exist mainly involving time and cost [68]. For example, according to Vig et al., most skin products require 2-3 weeks of culture time and significant lab resources; for example, approximately $\$ 13,000$ of lab resources are needed to produce enough of Epicel ${ }^{\mathrm{TM}}$ skin to cover $1 \%$ of the body's surface area [68]. Given the limitation of current techniques, research into more efficient 3D culture methods is important.

3D spheroids have recently risen to the forefront of research, mainly involving tumor cancer biology, brain organoids, angiogenesis sprouting, and drug testing applications [69]-[77]. Spheroids are essentially aggregates of cells stimulated to form 3D structures by a variety of culture methods including hanging drop, non-adhesive surfaces, spinner flask, and microcarrier beads (Figure 17) [77]. Spheroids have demonstrated success with numerous peer-reviewed papers [69]-[77]. Additionally, a number of groups have utilized the non-adherent culture conditions, with either low adherence $96-$ well culture plates or $1 \%$ agarose coating of flat-bottom 
wells [71]-[74]. Cells at an optimized seeding density were combined with methylcellulose (15$20 \%$ diluted in media) and seeded into non-adherent conditions [72], [73]. Methylcellulose functions as a binding agent to provide a high viscosity environment for optimal spheroid compaction [78]. After a week of culture, mature spheroids usually form and are ready for assessment [72], [73]. In addition, mixed culture spheroids are possible, with one group utilizing three cell types to form blood brain barrier spheroid organoids [70]. Spheroids were commonly assessed via immunostaining and fluorescence microscopy or histological embedding and sectioning [69]-[77]. Additionally, spheroids can be iteratively optimized by varying seeding density, methylcellulose concentration, culture time, and co-culture conditions among other variables. Given the relatively time-efficient protocols, reduced use of lab resources, and control over culture parameters, spheroids prove useful for investigation into skin applications.

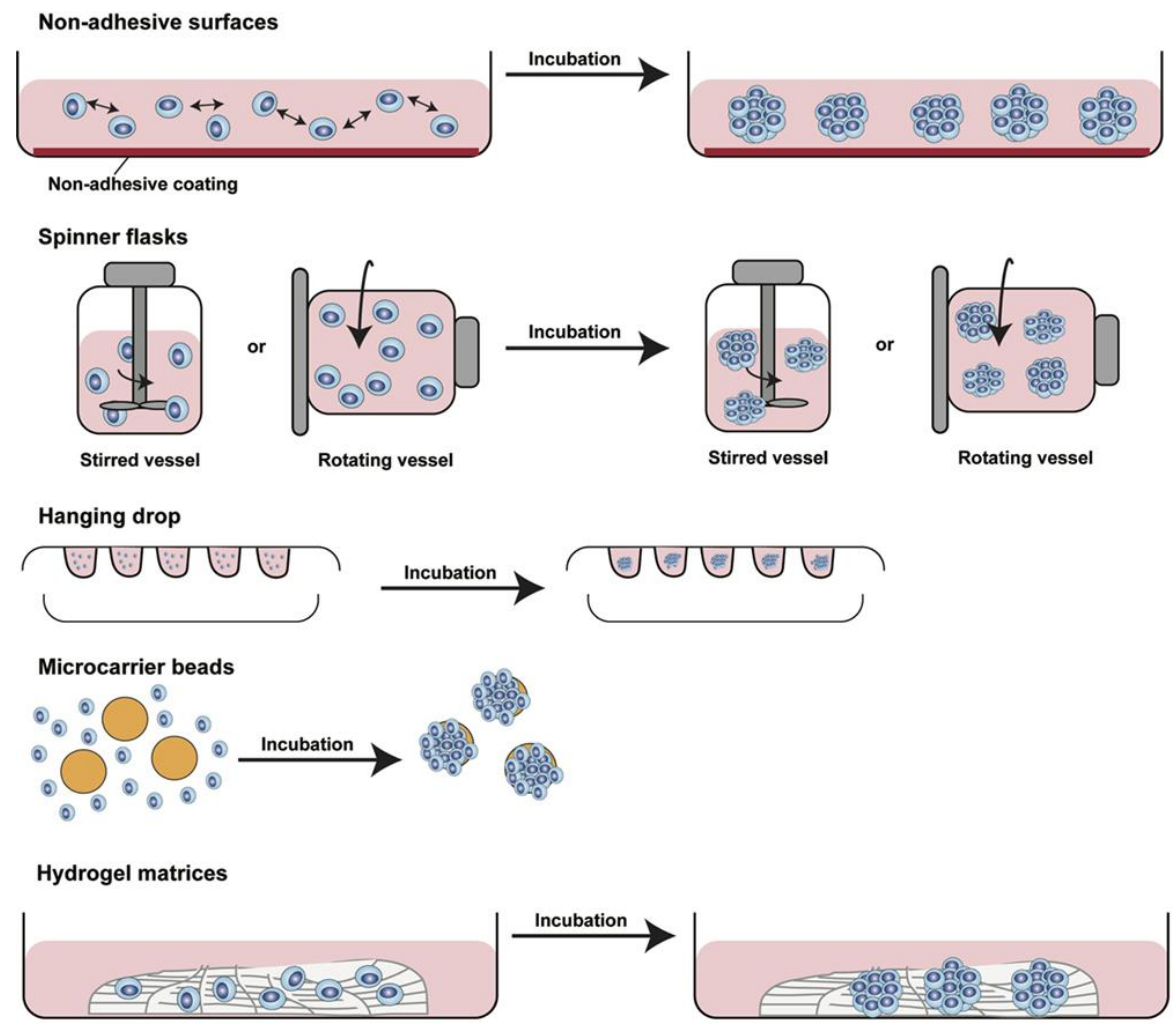

Figure 17: Methods of 3D Spheroid Formation [77].

Due to the historical success of sheet-based skin models (Figure 16), few research groups have developed skin spheroids [79], [80]. Some research groups have found success combining 
KRTs with dermal fibroblasts in a dual culture system [79]. Furthermore, Syntivia, a French biotechnology company, developed a skin model for in vitro cosmetic testing [80]. Following patent publication in August 2017, Syntivia created an epidermal keratinocyte-melanocyte coculture spheroid in Matrigel [80]. Their patent pointed to the highly optimized process and coculture method required for skin to stratify in a 3D spheroid structure [80]. Despite Syntivia's success, their Matrigel scaffold is limited in that the constituent proteins are highly uncharacterized, derived from mouse sarcoma (a xenograph and potentially immune eliciting substance), and varies significantly between product lots and batches [81]. Further research into a consistent skin cell spheroid model is warranted to determine if the model is physiologically relevant to previous 2D monolayer studies and gold standard sheet-based constructs. 


\subsection{Overview and scope of thesis}

Past research in the lab involving bovine milk SM and the p21 damage marker has demonstrated a photoprotective promise for SM. However, previous cell monolayer research has failed to demonstrate the mechanism of action for protection, link FSM with UVB, and recapitulate the 3D environment of skin. Thus, the goals of this thesis were three-fold and attempted to answer the following research question goals, each divided into separate subsets.

1.) Goal 1: Does FSM intracellular trafficking change with varying incubation times, and after UVB radiation?

a. Subset 1: Characterize FSM in KRT culture during live and fixed cell cultures using fluorescence confocal imaging

b. Subset 2: Assess the localization of FSM in normal and UVB-stressed KRTs under varying incubation times

c. Subset 3: Demonstrate functional equivalence of bovine milk SM to its fluorescent counterpart

2.) Goal 2: Do endogenous SM and ceramide levels fluctuate with exogenous FSM incubations? Is it possible to characterize, with fluorescence microscopy, the identity of the observed FSM signal over time?

a. Subset 1: Develop immunofluorescence staining protocols for both endogenous SM (lysenin) and endogenous ceramide

b. Subset 2: Characterize how endogenous SM and ceramide levels change with varying FSM incubation timepoints

3.) Goal 3: Are cell spheroids a viable platform to move future research from a $2 \mathrm{D}$ to $3 \mathrm{D}$ model?

a. Subset 1: Develop a method to culture NIH 3 T3 immortalized mouse fibroblast spheroids, for eventual incorporation of KRT cells and FSM 
b. Subset 2: Quantify dye penetration over various concentrations and incubation times

First, FSM was characterized in our model system of KRTs. A p21 immunofluorescence stain was employed as a substantial equivalence test to compare photoprotective benefits of FSM and bovine milk SM. The location and brightness of the stain was tracked both in live and $3.75 \%$ paraformaldehyde (PFA) fixed cells using confocal microscopy. The main purpose of localization quantification was to assess where the FSM stain is trafficked, and if fluorescence localization is perturbed with UVB exposure. Trafficking is valuable because different lipid metabolic processes occur in various cellular locations [27].

It was hypothesized that total intracellular FSM levels would initially increase when added to the cells, then decrease with hydrolysis to ceramide and exocytic recycling mechanisms. With UV exposure, the FSM levels would diminish further because UV would stimulate the hydrolysis mechanism and increase levels of ceramide. Enhanced intracellular ceramide would then increase apoptosis levels in a cell, contributing to FSM photoprotection.

Second, immunofluorescence staining protocols were developed for both endogenous SM (lysenin) and endogenous ceramide. The goal of incorporating these two stains was to assess, both qualitatively and quantitively, the location of endogenous SM and ceramide in a cell and if introduction of exogenous FSM disturbed lipid dynamics. Although not completed in this thesis, lysenin and ceramide stains could eventually be used to assess the fluctuations in localization and signal brightness after UVB exposure. Another future goal for immunostaining is to better characterize FSM by developing a repeatable and quantifiable fluorescence colocalization method for FSM, lysenin, and ceramide. As FSM is introduced and metabolized, it is unknown whether the fluorescence probe remains attached to a downstream metabolite (not a true sphingomyelin) or if the probe is cleaved and exocytosed from the cell. Measuring colocalization of lysenin with 
FSM and ceramide with FSM provide first steps towards understanding the degradation of the fluorescent probe attached to FSM.

Finally, the third main goal was to develop a culture method for 3D spheroids using 3T3 cells. The nuclei of $3 \mathrm{~T} 3$ spheroids were stained and imaged on the confocal microscope to assess dye penetration depth. The methods developed can be used to culture KRT-3T3 dual spheroids and quantify the effects of FSM incubation on a 3D culture system. 


\section{METHODS}

\subsection{Keratinocyte Cell Culture}

Primary Epidermal Keratinocytes (KRTs), Normal, Human, Neonatal Foreskin (HEKn, PCS-200-010, ATCC Manassas, VA) were cultured using aseptic technique in a $37^{\circ} \mathrm{C}, 5 \% \mathrm{CO}_{2}$ incubator prior to experiments. KRT media was made following ATCC guidelines by combining one Keratinocyte Growth Kit (PCS-200-040, ATCC, Manassas, VA) with one bottle of Dermal Cell Basal Medium (PCS-200-030, ATCC, Manassas, VA), and 0.5 mL of PenicillinStreptomycin-Amphotericin (PCS-999-002, ATCC, Manassas, VA). The mixture was then sterile filtered with a $0.22 \mu \mathrm{m}$ filter. KRTs were expanded in canted T-75 Falcon from Corning Tissue Culture Flasks (BD353136, VWR, Visalia, CA) and passaged at approximately 70\% confluence into 8-well Nunc Lab-Tek Chambered Coverglasses from Thermo Scientific (43300-774, VWR, Visalia, CA) for experiments. The 8-well plates were mounted on No. 1 borosilicate glass and allowed for inverted confocal imaging. During culture, the monolayer of cell was visually assessed via brightfield microscopy every 24-48 hours and media was exchanged every 48 hours.

\subsection{Sphingomyelin Treatment - BSM and FSM}

Bovine Milk SM or BSM (860063P-25mg, Avanti Polar Lipids, Alabaster, AL) was obtained in powdered form. The BSM was weighed and added to KRT media to create a $0.1 \%$ BSM solution. The powder was mixed in the media via sonication and vortexing to create a homogenous solution. The solution was finally passed through a $0.22 \mu \mathrm{m}$ filter to sterilize then stored in the refrigerator. The BSM solution was discarded on the expiration date of the media.

Fluorescent SM or C6-NBD Sphingomyelin (810218P-1mg, Avanti Polar Lipids, Alabaster, AL) was also acquired in powdered form then dissolved in ethanol to yield a $1 \mathrm{mM}$ stock solution. The stock was stored at $-20^{\circ} \mathrm{C}$ in a tightly sealed glass container protected from light until further use. To make the FSM media treatment administered to cells, an appropriate 
volume of ethanol-FSM stock was added to a 5uM Bovine Serum Albumin diluted in KRT media to yield a 5uM FSM final concentration.

When KRTs in the 8-well plates reached $70 \%$ confluence, approximately $200 \mu \mathrm{L}$ of SM treatment was added to each well. Cells were incubated for various times with the SM prior to UV radiation.

\subsection{UVB Irradiation}

UVB radiation $(302 \mathrm{~nm})$ at a dose of $40 \mathrm{~mJ} / \mathrm{cm}^{2}$ was administered to the cells in a twostep process. First, the average intensity of the UV lamp (95-0251-01, UVP, LLC, Upland, CA) was measured with a UV sensor (S120UV, ThorLabs, Newton, NJ) and connected power meter (PM100, ThorLabs, Newton, NJ). Figure 18 provides a visual representation of the UV lamp calibration and treatment administration under aseptic technique. All equipment was sprayed with 70\% Isopropyl Alcohol (IPA) prior to use in the culture hood.
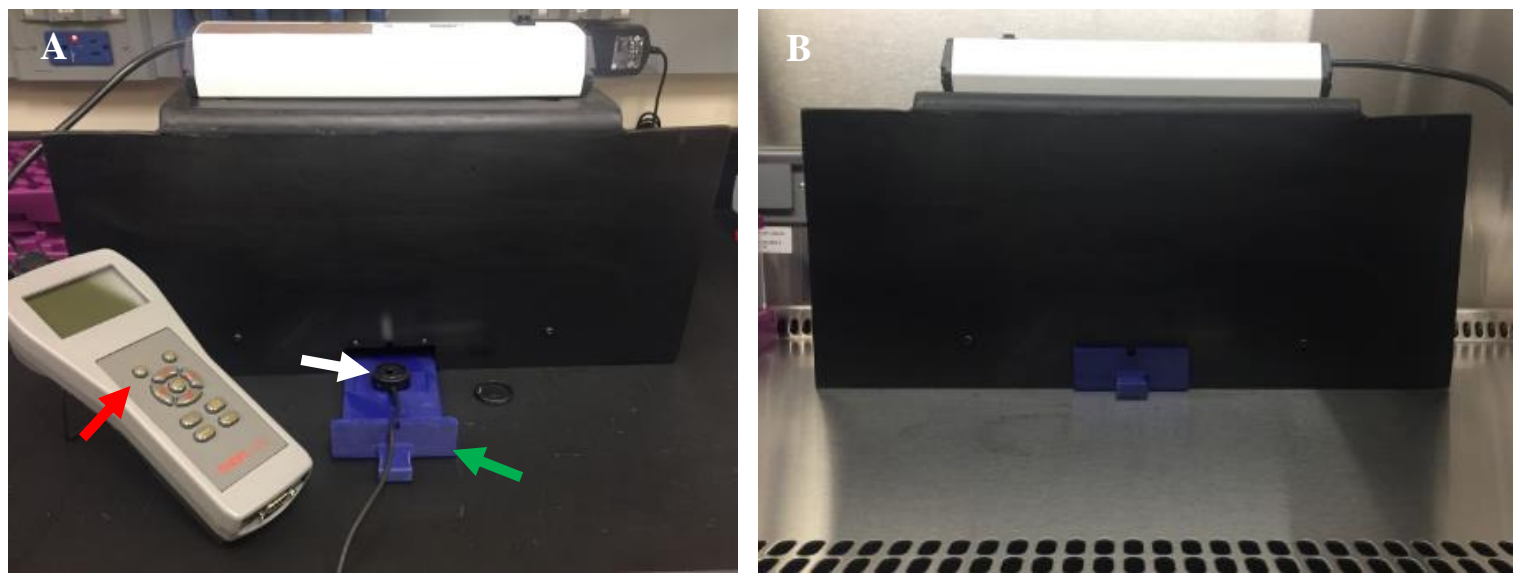

Figure 18: UVB Radiation. (A) Calibration of Lamp, sensor (white arrow), power meter (red arrow), ABS plastic tray (green arrow). The white UV lamp is shown atop the black containment box. The exposure time was calculated based on the desired dose and average intensity of the lamp. (B) UV calibration and treatment to cells were conducted inside the culture hood after spraying all equipment down with 70\% IPA. The cells were placed on the blue plastic tray and slid under the UV box/lamp. Spent media was replaced prior to UV. 
The exposure time of cells to UV required for the radiation dosage was calculated using the following equation [13]. The measured irradiance was calculated by determining the average intensity of the UV sensor during calibration, and dividing by the sensor area of $0.7088 \mathrm{~cm}^{2}$ [13]. The prescribed dose is equal to $40 \mathrm{~mJ} / \mathrm{cm}^{2}$. The exposure time is roughly 60 seconds.

$$
\text { Exposure Time (seconds) }=\frac{\text { Prescribed Dose }\left(\frac{\mathrm{mJ}}{\mathrm{cm}^{2}}\right)}{\left(\frac{1 \mathrm{~mW}}{1000 \mu \mathrm{W}}\right) * \text { Measured Irradiance }\left(\frac{\mu \mathrm{W}}{\mathrm{cm}^{2}}\right)}
$$

The UV lamp was housed in UV box constructed of Delrin plastic which contained the radiation and protected the user. The Acrylonitrile Butadiene Styrene (ABS) plastic tray was 3D printed to hold the sensor and 8-well culture plate, and ensure the system remains directly under the UV radiation source (green arrow Figure 18).

The second step of the UV process was direct administration to the cells. KRTs in 8-well plates were removed from the incubator and the spent media (or SM solutions) were aspirated and replaced with $200 \mu \mathrm{L}$ of fresh KRT media. The plates were placed on top of the tray, slid under the UV lamp, and exposed to UV for the calculated time (based on the dose and irradiance). After UV, cells were placed back in the incubator and visually assessed for signs of contamination.

Figure 19 provides an example plate layout used for SM and UV treatment.

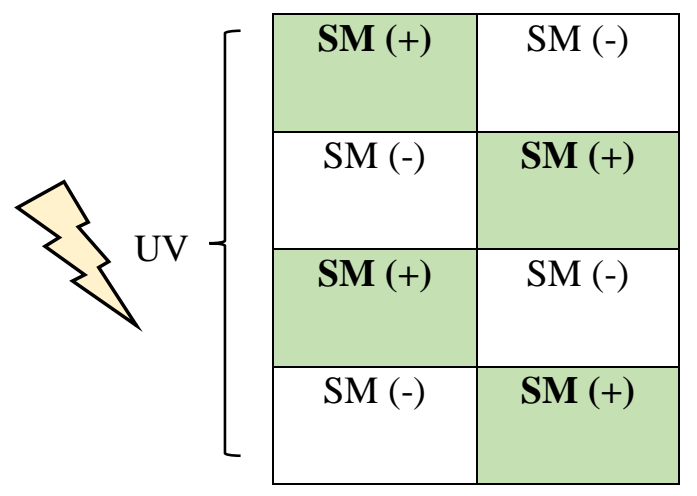

Figure 19: Typical Plate Distribution for SM and UV Treatments. SM was administered in a diagonal pattern with (+) meaning the presence of SM and (-) denoting the absence of SM. The entire plate was subjected to UV, as indicated by the lightning bolt. 
SM was directly added to wells in a diagonal pattern, and the entire plate was UV'ed. The diagonal pattern randomized the treatment locations and reduced bias introduced by positiondependent fluctuations in UV intensity.

\subsection{Cell Fixation and Staining}

After UV exposure, cells were fixed in $3.7 \%$ paraformaldehyde (PFA). The time after UV was dependent on the experimental treatment. The media was gently removed from the wells, and one wash was performed with a 1X Phosphate Buffer Solution (PBS). 200 $\mu$ L of PFA was pipetted in each well, and the plate was incubated for 15 minutes at room temperature. After fixation, cells were washed three times with PBS. After fixation, cells were subjected to various immunostaining protocols depending on the experimental objective.

\subsection{1 p21}

The p21 indirect immunostaining protocol was previously optimized by the lab [13]. Following BSM or FSM incubation and UV exposure, p21 immunostaining was conducted according to established protocol shown in Table I. 
Table I: p21 Immunostaining Protocol [13]

\begin{tabular}{|c|c|c|c|c|}
\hline Protocol Step & Reagent & Concentration & Exposure & Vendor Information \\
\hline Fixation & Paraformaldehyde & $3.7 \%$ & 15 min & $\begin{array}{c}\text { Electron Microscopy } \\
\text { Solutions (RT 15710) }\end{array}$ \\
\hline Permeabilization & Trixon X-100 & $0.1 \%$ & 20 min & Invitrogen HFH10 \\
\hline Blocking & $\begin{array}{c}10 \% \text { Normal Goat } \\
\text { Serum }\end{array}$ & $1 \%$ & $\begin{array}{c}\text { Overnight } \\
(\sim 12 \text { hrs) }\end{array}$ & $\begin{array}{c}\text { Life Technologies } \\
500622\end{array}$ \\
\hline Primary & $\begin{array}{c}\text { Anti-p21 antibody } \\
\text { (rabbit) }\end{array}$ & $1: 400$ & 9 Hours & Abcam ab18209 \\
\hline Secondary & $\begin{array}{c}\text { AF 647 (goat, } \\
\text { anti-rabbit) }\end{array}$ & $1: 400$ & 1 hour & $\begin{array}{c}\text { ThermoFisher } \\
\text { Scientific A-11008 }\end{array}$ \\
\hline Nuclear Staining & $\begin{array}{c}\text { Hoechst 34580 } \\
\text { Life Technologies } \\
\text { H21486 }\end{array}$ \\
\hline
\end{tabular}

The monolayer of each well was exposed to $200 \mu \mathrm{L}$ of $0.1 \%$ TritonX-100 diluted in PBS for 20 minutes to permeabilize the cell membrane and allow antibody entry into the nucleus. $200 \mu \mathrm{L}$ of a blocking solution, $10 \%$ goat serum diluted in PBS, was administered and allowed to incubate overnight at $4^{\circ} \mathrm{C}$. The blocking solution reduced non-specific binding of the primary and secondary while reducing image noise and enhancing signal to noise ratio. The next day, $200 \mu \mathrm{L}$ of 1:400 p21 antibody (raised in rabbit) diluted in PBS was added and incubated for 9 hours at $4^{0} \mathrm{C}$. Next, $200 \mu \mathrm{L}$ of 1:400 goat, anti-rabbit AF 647 secondary diluted in PBS was administered for 1 hour. Finally, a Hoechst 34580 stain, 0.03\% diluted in PBS was added to the well for 15 minutes. Between each step in the process, the previous reagent was washed off 3 times with PBS. Care was taken to gently administer the liquid treatments to each well in order to prevent washing off of cells.

\subsubsection{Lysenin}

In order to visualize endogenous sphingomyelin in a cell, indirect immunostaining was conducted. The protein Lysenin was used to bind on to sphingomyelin, then an anti-Lysenin antibody and secondary were introduced to fluorescently tag the lysenin (Table II) 
Table II: Lysenin Immunostaining Protocol

\begin{tabular}{|c|c|c|c|c|}
\hline Protocol Step & Reagent & Concentration & Exposure & Vendor Information \\
\hline Fixation & Paraformaldehyde & $3.7 \%$ & $15 \mathrm{~min}$ & $\begin{array}{l}\text { Electron Microscopy } \\
\text { Solutions (RT 15710) }\end{array}$ \\
\hline Permeabilization & Trixon X-100 & $0.1 \%$ & $20 \mathrm{~min}$ & Invitrogen HFH10 \\
\hline Blocking & $\begin{array}{c}\text { BSA } \\
\text { 10\% Normal Goat } \\
\text { Serum }\end{array}$ & $4 \% / 4 \%$ & $\begin{array}{l}\text { Overnight } \\
(\sim 12 \mathrm{hrs})\end{array}$ & $\begin{array}{c}\text { Spectrum Chemicals } \\
\text { AL } 135 \\
\text { Life Technologies } \\
500622\end{array}$ \\
\hline $\begin{array}{c}\text { Protein } \\
\text { Attachment to } \\
\text { SM } \\
\end{array}$ & $\begin{array}{l}\text { Lysenin from } \\
\text { Eisenia foetida }\end{array}$ & $1 \mu \mathrm{g} / \mathrm{mL}$ & 2 hours & $\begin{array}{l}\text { Millipore Sigma } \\
\text { L3038 }\end{array}$ \\
\hline Primary & $\begin{array}{l}\text { Anti-Lysenin pAb } \\
\text { (rabbit) }\end{array}$ & $1: 1000$ & 1 hour & $\begin{array}{c}\text { Creative Diagnostics } \\
\text { DPAB214905 }\end{array}$ \\
\hline Secondary & $\begin{array}{l}\text { AF } 594 \text { (goat, anti- } \\
\text { rabbit) }\end{array}$ & $1: 300$ & 1 hour & $\begin{array}{c}\text { ThermoFisher } \\
\text { Scientific A11012 }\end{array}$ \\
\hline Nuclear Staining & Hoechst 34580 & $0.03 \%$ & $15 \mathrm{~min}$ & $\begin{array}{c}\text { Life Technologies } \\
\text { H21486 }\end{array}$ \\
\hline
\end{tabular}

After fixation and permeabilization, the cells were blocked overnight at $4{ }^{0} \mathrm{C}$ with $4 \%$ BSA and 4\% Goat Serum solution, abbreviated 4\%BSA/4\%Goat, diluted in PBS. This blocking solution was optimized specifically for lysenin and dual staining with ceramide to prevent nonspecific primary and secondary binding. Tween-20 was temporally tested in the blocking buffer to further reduce non-specific primary binding, but it was ultimately removed when the FSM signal was washed out.

Lysenin from Eisenia foetida (L3038-50UG, Millipore Sigma, St. Louis, MO) was purchased in powder form and reconstituted in $2 \%$ BSA in PBS to make a $10 \mu \mathrm{g} / \mathrm{mL}$ stock. Unused stock was aliquoted and stored at $-20^{\circ} \mathrm{C}$. The lysenin was further diluted in PBS and incubated with cells at a $1 \mu \mathrm{g} / \mathrm{mL}$ optimized concentration for 2 hours at room temperature. Next, a rabbit anti-lysenin polyclonal antibody (DPAB214905, Creative Diagnostics, Shirley, NY) was diluted 1/1000 in blocking solution and incubated for 1 hour at room temperature. The secondary AF594 Goat Anti-Rabbit (A11012, ThermoFisher Scientific, Waltham, MA) was diluted 1:300 in PBS and incubated for 1 hour at room temperature. Lastly, the nucleus was stained with $0.03 \%$ 
concentration of Hoechst 34580 for 15 minutes at room temperature. Between each protocol step, the previous reagent was washed three times with PBS.

\subsubsection{Ceramide}

An indirect immunofluorescence staining assay was utilized to fluorescently visualize ceramide in a cell. Table III details the list of reagents and optimized staining procedure. After fixation and permeabilization, the cells were blocked overnight at $4{ }^{\circ} \mathrm{C}$ with $4 \% \mathrm{BSA}$ and $4 \%$ Goat Serum solution, abbreviated 4\%BSA/4\%Goat, diluted in PBS. A mouse anti-ceramide monoclonal antibody (C8104, Millipore Sigma, St. Louis, MO) was diluted 1/400 in blocking solution and incubated for 9 hours at $4^{0} \mathrm{C}$. The secondary AF 647 Goat Anti-Mouse (A21235, ThermoFisher Scientific, Waltham, MA) was diluted 1:300 in PBS and incubated for 1 hour at room temperature. Lastly, the nucleus was stained with $0.03 \%$ concentration of Hoechst 34580 for 15 minutes at room temperature. Between each protocol step, the previous reagent was washed three times with PBS.

Table III: Ceramide Immunostaining Protocol

\begin{tabular}{|c|c|c|c|c|}
\hline Protocol Step & Reagent & Concentration & Exposure & Vendor Information \\
\hline Fixation & Paraformaldehyde & $3.7 \%$ & $15 \min$ & $\begin{array}{l}\text { Electron Microscopy } \\
\text { Solutions (RT 15710) }\end{array}$ \\
\hline Permeabilization & Trixon $\mathrm{X}-100$ & $0.1 \%$ & $20 \mathrm{~min}$ & Invitrogen HFH10 \\
\hline Blocking & $\begin{array}{c}\text { BSA } \\
10 \% \text { Normal Goat } \\
\text { Serum }\end{array}$ & $4 \% / 4 \%$ & $\begin{array}{l}\text { Overnight } \\
(\sim 12 \mathrm{hrs})\end{array}$ & $\begin{array}{c}\text { Spectrum Chemicals } \\
\text { AL } 135 \\
\text { Life Technologies } \\
500622 \\
\end{array}$ \\
\hline Primary & $\begin{array}{c}\text { Anti-Ceramide } \\
\text { mAb (mouse) }\end{array}$ & $1: 400$ & 9 hours & $\begin{array}{l}\text { Millipore Sigma } \\
\text { C8104 }\end{array}$ \\
\hline Secondary & $\begin{array}{c}\text { AF } 647 \text { (goat, anti- } \\
\text { mouse) }\end{array}$ & $1: 300$ & 1 hour & $\begin{array}{c}\text { ThermoFisher } \\
\text { Scientific A21235 }\end{array}$ \\
\hline Nuclear Staining & Hoechst 34580 & $0.03 \%$ & $15 \mathrm{~min}$ & $\begin{array}{c}\text { Life Technologies } \\
\text { H21486 }\end{array}$ \\
\hline
\end{tabular}




\subsubsection{Dual Staining}

Cells were co-stained for both lysenin and ceramide. Cells were fixed and permeabilized, followed by overnight blocking at $4^{0} \mathrm{C}$ with $4 \% \mathrm{BSA} / 4 \%$ Goat diluted in PBS. Next, the lysenin protein then subsequent lysenin antibody were introduced to the cells according to established protocol. Then the ceramide antibody was incubated for 9 hours, followed by simultaneous secondary incubations (AF 647 goat anti-mouse and AF 594 goat anti-rabbit) diluted in PBS and introduced at the same time at their respective concentrations. Finally, the nuclei were stained with Hoechst. Sufficient washing steps were performed between each step in the protocol.

\subsubsection{Nile Red}

Nile Red powder (N3013-100MG, Millipore Sigma, St. Louis, MO) was reconstituted in methanol to from a $1 \mathrm{mg} / \mathrm{mL}$ stock concentration which was stored long-term at $20^{\circ} \mathrm{C}$. The stock was further dissolved in PBS to make a $5 \mu \mathrm{M} / \mathrm{mL}$ solution. Cells were exposed to Nile red for 15 minutes than washed three times with PBS prior to imaging.

\subsection{Spheroid Cultivation, Staining, and Manipulation}

Spheroids were formed by adding NIH-3T3 cells to methylcellulose binding agent. The cells plus methylcellulose were seeded into non-adherent U-well plates and cultured for 3-5 days prior to cultivation, staining, and confocal imaging. To reduce the cost associated with using the U-well plates, flat bottom U-wells were coated with agarose prior to cell seeding and culture. A co-culture of 3T3 and KRTs (stained with CellTracker Red) were also cultured in U-wells as a first step towards promoting KRT stratification into spheroids.

\subsubsection{T3 Cell Culture}

NIH 3 T3 Fibroblasts were cultured using aseptic technique in a $37^{\circ} \mathrm{C}, 5 \% \mathrm{CO}_{2}$ incubator prior to experiments. 3T3 media was made by adding Fetal Bovine Serum (F8317, Millipore 
Sigma, St. Louis, MO) to DMEM media (12001-000, VWR, Visalia, CA), and PenicillinStreptomycin-Amphotericin (PCS-999-002, ATCC, Manassas, VA). The media was sterile filtered and stored at $4^{0} \mathrm{C}$ for up to one month.

\subsubsection{Methylcellulose}

A $2 \%$ weight/volume solution was chosen for the concentration of methylcellulose based on previous literature [72], [73]. 5 grams of methylcellulose powder (M7140-100G, Millipore Sigma, St. Louis, MO) was weighed and added to $250 \mathrm{~mL}$ of Distilled $\mathrm{H}_{2} \mathrm{O}$ and slowly brought to a boil for 5-10 minutes. A stir bar was added to enhance mixing. The solution was agitated and heated until all methylcellulose particles were wet and evenly dispersed. The solution was immediately sterilized for 16 minutes at $121^{\circ} \mathrm{C}$ and 15 Pounds per Square Inch (PSI) steam pressure. The sterile methylcellulose was stored at room temperature to facilitate complete dispersion.

\subsubsection{Cell Seeding}

Cells were mixed with $20 \%$ methylcellulose solution diluted in cell media. The seeding density was varied per experiment. $200 \mu \mathrm{L}$ of cell-20\% methylcellulose was pipetted into each well of 96-well Corning ${ }^{\circledR}$ spheroid microplates with ultra-low attachment surface (CORN4520, VWR, Visalia, CA) and incubated for 3-5 days. Spheroids were visually assessed for growth and health every two days.

For co-culture dual experiments with $3 \mathrm{~T} 3$ fibroblasts and KRTs, the seeding density ratio was matched by the media concentration. In some concentrations, the amount of methylcellulose was increased to $40 \%$. 


\subsubsection{Agarose}

Agarose (V0710-25G, VWR, Visalia, CA) was used to coat the bottom of 96-well flat bottom plates to make the surface non-adherent and promote cell aggregation into spheroids. Agarose plates are a potentially less expensive alternative to the Corning® ultra-low attachment spheroid microplates.

Each well was coated with $100 \mu \mathrm{L}$ of $1 \%$ sterile agarose. 0.1 gram of agarose powder was added to $10 \mathrm{~mL}$ of distilled $\mathrm{H}_{2} \mathrm{O}$ and microwaved for about 1 minute until the agarose powder was dissolved. The heated solution was immediately sterilized with a $0.22 \mu \mathrm{m}$ filter then dispensed quickly into each well. Efficiency and rapid pipetting was required before the agarose solution cooled and solidified in the pipet tip. The agarose in each well was allowed to gel for at least 1 hour at room temperature before seeding the cell-20\% methylcellulose solution.

\subsubsection{Manipulation, Staining, Plating}

After 3-5 days of culture, each individual spheroid was removed from the well with a $1000 \mu \mathrm{L}$ pipet tip and placed into a $1 \mathrm{~mL}$ conical tube. Generally, 3-5 spheroids were pipetted into each tube. Given the density of the spheroids, they naturally accumulated at the bottom of the tube, and the residual media and/or stains were carefully removed/added without disturbing the spheroids at the bottom. One PBS wash was performed by slowly introducing $200 \mu \mathrm{L}$ to each conical tube, pipetting gently to mix, then removing the liquid.

Spheroids were fixed with $3.75 \%$ PFA for 20 minutes, then rinsed three times with PBS to remove residual fixative. Hoechst stain in PBS was introduced at various concentrations to the spheroids by adding $200 \mu \mathrm{L}$ to each tube and gently mixing. After the staining treatment, Hoechst

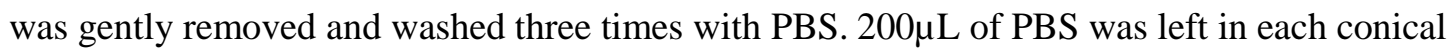
between staining and imaging. 
Immediately prior to imaging, the stained and washed spheroids were plated in glassbottom 8 -well plates by aspirating the $200 \mu \mathrm{L}$ PBS with a $1000 \mu \mathrm{L}$ pipet tip. The tip was pushed towards the bottom to ensure all the spheroids were aspirated into the tip. The entire $200 \mu \mathrm{L}$ liquid in the pipet was quickly ejected into one well of the 8-well plate. This process was performed rapidly to prevent spheroids from sticking to the plastic pipet tip. The spheroids sink to the bottom of the plate and are easily visualized under the confocal microscope.

\subsection{Confocal Imaging}

Images were obtained with an Olympus FluoView FV1000 confocal microscope (Olympus America, Centerville, PA) with FluoView program. The software applied DAPI for Hoechst, 488 laser (for Alexa Fluor 488), 559 laser (for Alexa Fluor 594), and 635 laser (for Alexa Fluor 647). The 60X and 100X oil objectives were used to yield sufficient resolution for fluorescence distribution analysis. The laser scanning speed was set to $4.0 \mu \mathrm{s} /$ pixel and image size was 512x512. Sequential was checked for multiple laser images.

Images were deliberately taken in the center of the well to ensure equal distribution of UV radiation exposure. Z-stacks with a $1 \mu \mathrm{m}$ step size were taken to provide a more realistic 3D image. The following settings were maintained between experiments used for quantification purposes (Table IV and V).

Table IV: Fixed Trafficking with FSM and Post-UV

\begin{tabular}{|c|c|c|}
\hline Laser and Power & Stain & PMT Sensitivity \\
\hline $488(10 \%)$ & FSM & $540 \mathrm{HV}$ \\
\hline
\end{tabular}

Table V: Dual Staining with FSM, Lysenin, Ceramide

\begin{tabular}{|c|c|c|}
\hline Laser and Power & Stain & PMT Sensitivity \\
\hline $488(30 \%)$ & FSM & $645 \mathrm{HV}$ \\
\hline $559(30 \%)$ & AF 594 (Lysenin) & $830 \mathrm{HV}$ \\
\hline $635(30 \%)$ & AF 647 (Ceramide) & $715 \mathrm{HV}$ \\
\hline
\end{tabular}




\subsection{Image Analysis}

The software used for image analysis was ImageJ/Fiji, a public software based on the Java programming language and developed by the National Institutes of Health. MATLAB was also used as a component of the 2D Fluorescence Distribution analysis, and for quantifying areas under the resulting curves.

\subsubsection{P21 Ratios}

To demonstrate a reduction in p21 DNA damage marker expression in SM-treated cells, the ratio of $\mathrm{p} 21$ positive to total cells was calculated. $\mathrm{P} 21$ positive cells exhibited bright nuclear fluorescence compared to cytoplasm expression. For each well, the number of positive p21 cells was manually counted with an ImageJ cell counter, then compared to the overall number of cells.

\subsubsection{D Fluorescence Distribution}

A fluorescence distribution macro, developed previously by another student in the lab, was used to analyze all fluorescence distributions. Appendix A includes the code. Raw confocal images in .oib format were collected from the Fluoview imaging system and opened into ImageJ/Fiji. Color channels were separated. When analyzing z-stacks, an image representative of the middle of the cell was selected (as determined by a clear nuclear signal, and distinct absence of FSM signal colocalization in the nucleus). Each nucleus in the image was blurred (Gaussian), made binary, outlined, and counted. The borders of the FSM Region of Interest (ROI) were identified in a similar manner, and the nuclei was subtracted from the FSM ROI (so that only the cytoplasm was included). All pixels outside the cytoplasm and nuclei ROI were set to zero.

A rotation ROI was run by finding the center of the ROI, rotating a line 360 degrees about the center, and measuring the profile plot distribution across the line for every 6 degree angle increment. In total, 60 profile plots were generated for each cell. Because the nucleus was 
not exactly centered in all cells, the profile plots from nucleus to plasma membrane were not the same length and number of pixels. Therefore, MATLAB was used to average all 60 profile plots and normalize by interpolating data points (linear approximation between pixels) so that all plots had the same number of points ("0" through " 100 " with "0" and " 100 " representing the center of the nucleus and edge of the cytoplasmic ROI respectively). Within each image, cell profile plots were averaged, then all images within the same well were averaged. Finally, all wells within each treatment were averaged to yield a mean gray value fluorescence distribution versus distance from the nucleus. Standard errors were calculated based on well to well variability within each treatment.

Relative amount of intracellular fluorescence was calculated by finding the area under each fluorescence distribution curve with the cumulative trapezoidal numerical integration function (CUM TRAPZ). The area was used as a method of comparison for fluorescence distribution curves.

\subsubsection{Depth of Dye Penetration into Spheroids}

The depth of Hoechst dye penetration was calculated by first subtracting all stack slices below a consistent threshold value used for all images. Then each stack was normalized and divided into 10 equal increments (i.e. one increment $=$ total height/10) stepping through the entire stack from start to finish. The entire stack was z-projected and an ROI outline of the spheroid was generated with an ImageJ macro. For each increment slice, the fluorescence distribution codes were used with the same ROI generated in the previous step for all increment images. Finally, MATLAB was used to find the area under the fluorescence curves for each slice in order to determine the dye content over the depth of the spheroid. 


\section{SUMMARY OF EXPERIMENTS}

All conducted experiments are listed in Table VI according to the thesis project goals.

Table VI: Thesis Goals for Characterizing FSM, Staining Protocols, and 3D Cell Spheroids

\begin{tabular}{|c|c|c|}
\hline Goal & Summary of Experiments & $\begin{array}{l}\text { Thesis } \\
\text { Section }\end{array}$ \\
\hline \multirow{6}{*}{$\begin{array}{l}1 \\
\text { Characterize } \\
\text { FSM }\end{array}$} & $\begin{array}{l}\text { Initial FSM Imaging } \\
\text { Goal: Validate protocol methods for } 5 \mu \mathrm{M} \text { FSM }\end{array}$ & 4.1 \\
\hline & $\begin{array}{l}\text { Live FSM Intracellullar Trafficking } \\
\text { Goals: Visualize FSM distribution differences over various FSM } \\
\text { incubations; assess suitability of live imaging }\end{array}$ & 4.2 \\
\hline & $\begin{array}{l}\text { FSM Temperature Dependence } \\
\text { Goal: Assess temperature-dependent FSM uptake }\end{array}$ & 4.3 \\
\hline & $\begin{array}{l}\text { Fixed FSM Trafficking with Variable Incubation Times } \\
\text { Goal: Quantify FSM distribution differences over several FSM } \\
\text { incubation times in fixed cells }\end{array}$ & 4.4 \\
\hline & $\begin{array}{l}\text { Fixed FSM Trafficking with UV } \\
\text { Goal: Detect UV perturbations in FSM distribution }\end{array}$ & 4.5 \\
\hline & $\begin{array}{l}\text { P21 Ratios for BSM and FSM Comparison } \\
\text { Goal: Demonstrate functional equivalence of p } 21 \text { photoprotection } \\
\text { for FSM and BSM }\end{array}$ & 4.6 \\
\hline \multirow{6}{*}{$\begin{array}{l}2 \\
\text { Develop Staining } \\
\text { Protocols }\end{array}$} & $\begin{array}{l}\text { FSM with Nile Red } \\
\text { Goal: Determine suitability of Nile red stain }\end{array}$ & 4.7 \\
\hline & $\begin{array}{l}\text { Lysenin Stain Optimization } \\
\text { Goal: Determine optimal staining parameters }\end{array}$ & 4.8 \\
\hline & $\begin{array}{l}\text { Ceramide Stain Optimization } \\
\text { Goal: Determine optimal staining parameters }\end{array}$ & 4.9 \\
\hline & $\begin{array}{l}\text { Dual Lysenin, Ceramide, and FSM Staining } \\
\text { Goal: Validate protocol methods for dual immunofluorescence }\end{array}$ & 4.10 \\
\hline & $\begin{array}{l}\text { Troubleshooting FSM Degradation after Dual Treatment } \\
\text { Goal: Identify one reason for FSM degradation dilemma }\end{array}$ & 4.11 \\
\hline & $\begin{array}{l}\text { Dual Lysenin, Ceramide, and FSM Fluorescence Trafficking } \\
\text { Goal: Quantify Lysenin, Ceramide, and FSM distribution } \\
\text { differences over several FSM incubation times in fixed cells }\end{array}$ & 4.12 \\
\hline \multirow{4}{*}{$\begin{array}{l}3 \\
\text { Cultivate 3D } \\
\text { Spheroids }\end{array}$} & $\begin{array}{l}\text { KRT and } 3 \text { T3 Single Culture } \\
\text { Goal: Validate spheroid protocol methods }\end{array}$ & 4.13 \\
\hline & $\begin{array}{l}\text { KRT and 3T3 Dual Culture } \\
\text { Goal: Assess KRT-3T3 dual spheroids }\end{array}$ & 4.14 \\
\hline & $\begin{array}{l}3 \text { T3 Culture with Agarose-coated Wells } \\
\text { Goal: Test agarose coating, Triton X and FSM incubations }\end{array}$ & 4.15 \\
\hline & $\begin{array}{l}\text { Quantification of Spheroid Dye Penetration } \\
\text { Goal: Quantify dye penetration into spheroids }\end{array}$ & 4.16 \\
\hline
\end{tabular}




\subsection{Initial FSM Imaging}

\subsubsection{Summary and Objectives}

The goal of the first FSM experiment was proof-of-concept to demonstrate that FSM at a $5 \mu \mathrm{M}$ concentration is detectable by confocal imaging and yields a bright signal with low noise. This concentration is consistent in literature, with research groups employing either a $5 \mu \mathrm{M}$ or $4 \mu \mathrm{M}$ FSM concentration [59], [65], [82]-[84]. Furthermore, ThermoFisher's product guidelines for fluorescently labeled sphingolipids specified complexing $5 \mu \mathrm{M}$ FSM with $5 \mu \mathrm{M}$ BSA in PBS [82]. Therefore, a $5 \mu \mathrm{M}$ FSM diluted in $5 \mu \mathrm{M}$ BSA (in PBS) was chosen as the starting concentration for all experiments.

The past FSM literature imaged live cells to visualize trafficking and intracellular localization [58], [59], [84], and fixing cells prior to imaging was not addressed. However, research with Bovine Milk SM (BSM) at the Cal Poly Bioimaging Lab involved cell fixation prior to imaging [13], [14]. Therefore, to bridge the gap between FSM and BSM research, a secondary goal was to fix FSM-treated cells with 3.75\% PFA to determine if the exogenous FSM signal was conserved after fixation (i.e. FSM can survive cell fixation).

$200 \mu \mathrm{L}$ of $5 \mu \mathrm{M}$ FSM diluted in $5 \mu \mathrm{M}$ of BSA (in PBS) was introduced to a monolayer of live KRT cells cultured in glass coverslip 8 -well plates and incubated at $37^{\circ} \mathrm{C}$ for 1 hour. After incubation, the FSM media was removed and washed gently with PBS. Samples were either imaged live or fixed in $3.75 \%$ PFA immediately prior to imaging. Confocal images were taken on the 40x and 100x objective.

\subsubsection{Results}

Figure 20 includes images of the first FSM experiment. Figure 20A depicts fixed KRT cells with FSM, while Figures 20B-D involve live cell imaging without 3.75\% PFA fixation. The 
FSM signal for both fixed and live cells appeared robust with an apparent high signal to noise ratio. Visually, no differences in signal brightness was visualized between the fixed and live cells.

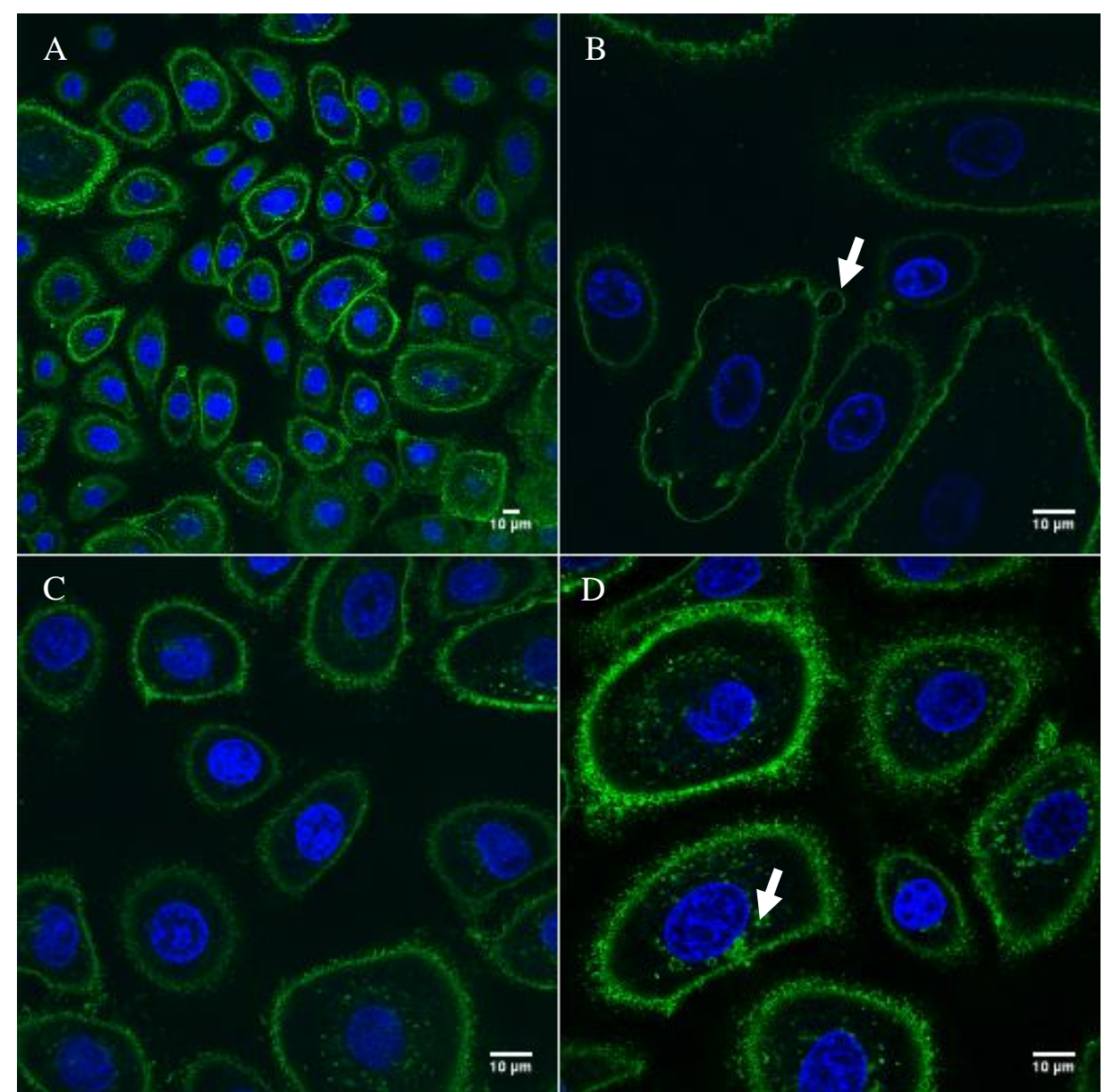

Figure 20: First FSM Study. (A) Fixed cells at 40x objective demonstrating that FSM signal survives PFA fixation, but a higher objective is a better indicator of intracellular fluorescence distribution. (B) Live cells at 100x objective showing blebbing (white arrow) from endocytosis/exocytosis, a mechanism for cellular FSM entry/exit. (C) Time image of live cells at

100x objective. FSM is predominantly concentrated in the plasma membrane, with some intracellular accumulation. (D) Time image, 100x objective, with intracellular distribution (white arrow) of FSM into cells.

Figure 20B-D were also taken on a higher confocal microscope objective (100x oil) compared to Figure 20A (40x oil). The higher magnification with larger numerical aperture (N.A.) enabled better visualization of intracellular FSM distribution. As shown by the white arrow in Figure 20D, clusters of fluorescence around the nucleus were visualized with the 100x objective. In addition, cellular phenomena like blebbing were observed, as demonstrated in 
Figure 20B. Blebbing, plasma membrane protrusions during endocytosis and exocytosis of lipid and cellular byproducts, is a scientifically characterized process. The blebbing observed during imaging most likely occurred as cells endocytose, metabolize, and exocytose exogenous lipids like FSM.

\subsubsection{Discussion}

The previously described experiment demonstrates that FSM incubation in KRT cell monolayers yields a bright and easily detectable signal. Furthermore, FSM can undergo fixation without a significant change in brightness or visual appearance. In addition, a higher objective with larger N.A., like the 100x, yields better resolution and is the objective of choice for future trafficking experiments involving intracellular fluorescence visualization. Lastly, two phenomena were visualized, both supported by literature [58], [60], [63], [84]. First, blebbing - protrusions of the plasma membrane - was observed as a potential indicator of FSM endocytosis or exocytosis. The cell accumulated FSM from its external supply in the media, then released its contents back into the environment. Endocytosis and exocytosis potentially mediate lipid homeostatic levels and have been characterized in scientific literature [63]. Furthermore, FSM accumulated in the plasma membrane of the KRT cells, then moved intracellular as clusters of fluorescence around the nucleus. The intracellular migration of FSM to regions close to the nucleus is also documented in literature [58], [60], [84]. Both phenomena observed in this first FSM study - blebbing and intracellular fluorescence migration - are important because they mirror published results and demonstrate that the current protocols (i.e. $5 \mu \mathrm{M}$ concentration, solution preparation, incubation with cells, and imaging) are sufficient to obtain scientifically relevant results. 


\subsection{Live FSM Intracellular Trafficking}

\subsubsection{Summary and Objectives}

In the context of this thesis, trafficking is defined as the fluorescence distribution of FSM both inside and along the plasma membrane of a cell. Differences in FSM trafficking were compared and qualitatively assessed among image treatments by location (i.e. near the nucleus versus concentrated in the plasma membrane) and/or in brightness of signal (correlated with the amount of FSM present in a specific location). Location could then be correlated with metabolic activity [27]. The main goal of the following experiment was to determine qualitatively if FSM trafficking in live KRT cell cultures changed as a result of the $37^{\circ} \mathrm{C}$ incubation time. It is important to note that cultures were not washed with PBS prior to imaging because the goal of this study was to assess the native in vitro FSM trafficking dynamics during continuous FSM incubations.

A secondary goal was to assess the suitability of live cell confocal imaging for FSM trafficking experiments. Live imaging presents a method to visualize live FSM trafficking within the same cell culture system. However, live microscopy can yield challenges which can affect image quality and cell phenotype. For example, continuous laser exposure during time scans of the same cell can photobleach the signal and yield a decrease in fluorescence brightness.

For the experimental procedure, a $37^{\circ} \mathrm{C}$ FSM incubation was used because previous experiments demonstrated FSM entry was temperature-dependent and a $37^{\circ} \mathrm{C}$ incubation was

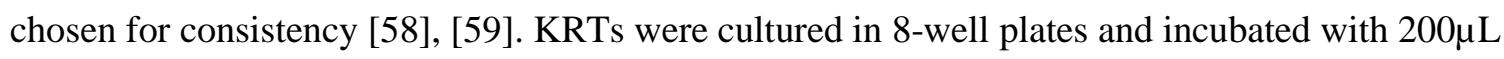
of $5 \mathrm{uM}$ of FSM for 10 minutes, 30 minutes, 1 hour, 2 hours, 3 hours, 4 hours, 12 hours, and 24 hours. FSM media was not removed prior to imaging in order to probe the cell conditions during a continuous FSM incubation. Cells were then imaged on the confocal at 100x objective, and both static and time-lapse images were acquired. 


\subsubsection{Results}

For this experiment, FSM was continuously incubated for 10 minutes, 30 minutes, 1 hour, 2 hours, 3 hours, 4 hours, 12 hours, and 24 hours. The 488 laser was used at 15\% power and 611 PMT sensitivity. Timescan images (sequential 2D images over a specified time range and laser pulse) were obtained for each timepoint. Specifically, each treatment Region of Interest (ROI) was imaged for 5 minutes, with 5 seconds between each laser pulse for a total of 60 images). Even after only 10 minutes of incubation, FSM was observed both at the plasma membrane and in the cytoplasm (Figure 21).
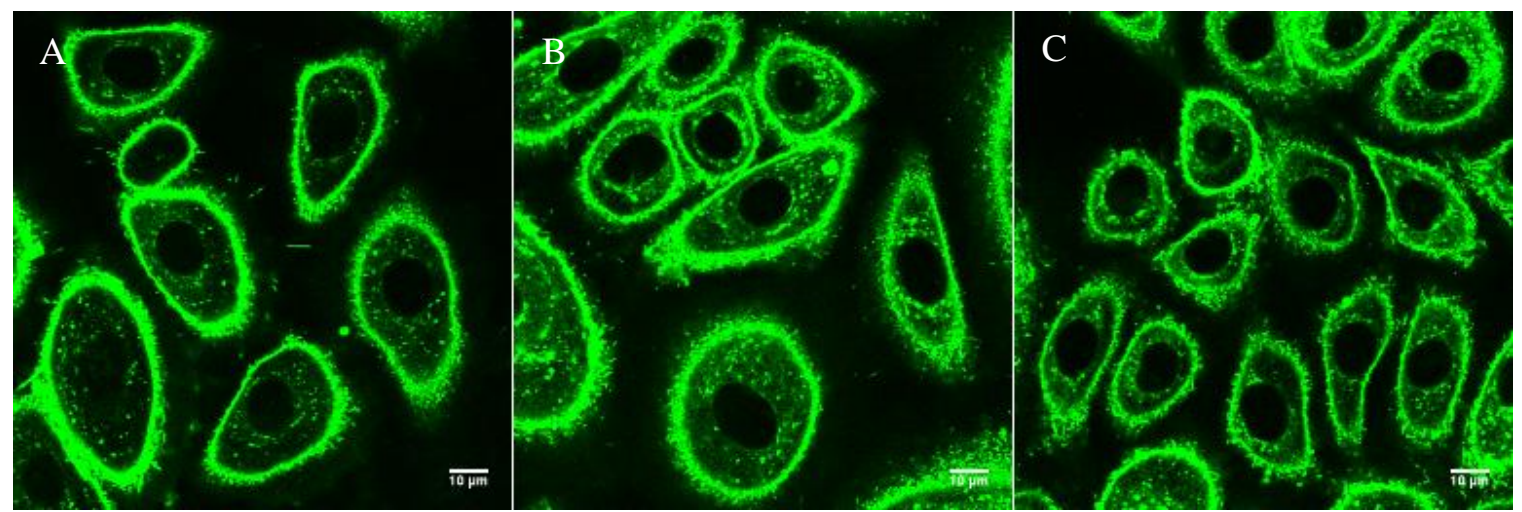

Figure 21: Representative Images for Live FSM Trafficking with Variable Incubation

Times. (A) 10 Minutes (100x), (B) 1 Hour (100x), (C) 12 Hours (100x). No difference in trafficking was observed between all timepoints, suggesting endocytosis and exocytosis homeostasis is reached early in the cell. The plasma membranes had a "shaggy" or coarse FSM staining because cells were directly probed during FSM media incubation and a wash step with

PBS was not performed prior to imaging.

More specifically, a bright plasma membrane staining was observed, and the staining was more intense compared to the first experiment. The reason for this could be due to the lack of FSM media washing prior to imaging. In the previous experiment, one gentle PBS wash was performed to remove residual extracellular FSM staining. The increase in plasma membrane fluorescence and coarse "shaggy" FSM appearance could be due to residual FSM weakly adherent to the plasma membrane that is usually washed off with PBS rinses. 
No qualitative change in fluorescence distribution or intensity was observed for all the timepoints. Whitelight overlaid with AF 488 images indicated that fluorescence distribution seemed to locate around the nucleus in the Golgi bodies, an observation which is supported by literature [58], [60], [84]. Figure 22 includes one static image of a 3 hour incubation, which serves as a representative image for all timepoints.

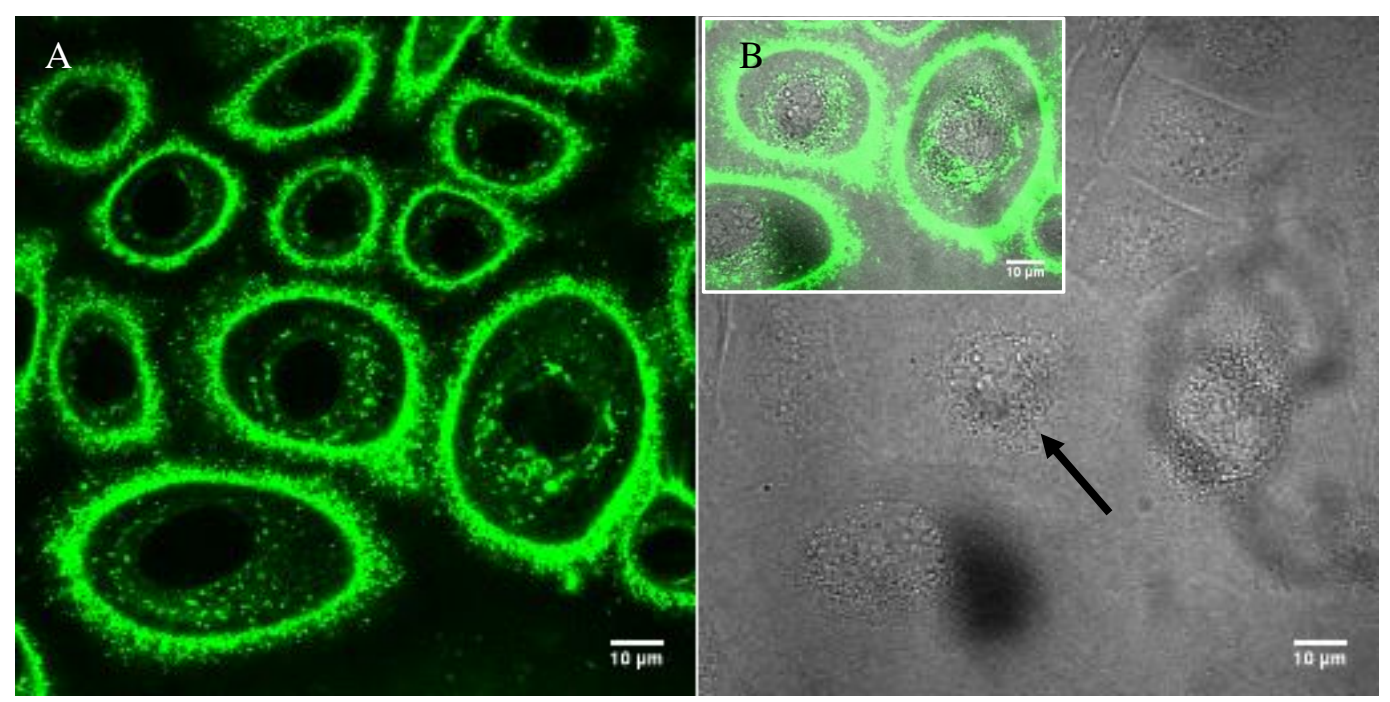

Figure 22: Three Hour Incubation Live Cells with FSM and DIC. Representative image of all timepoints (10 minutes -24 hour incubation) at 100x objective. Relatively consistent staining was observed among all timepoints. Intracellular fluorescence was concentrated around the nucleus in what appeared to be Golgi bodies (black arrow). Inset is an overlay of AF 488 (A) and DIC (B - whitelight).

While taking timescans, significant photobleaching was observed. In addition, the cells appeared to move during the scan. In some cases, the cells shifted upwards and away off the coverglass. To demonstrate observed cell movement and photobleaching during imaging, Figure 23 shows an image at the beginning of the scan (Figure 23A), and after 5 minutes of imaging with 5 second pauses between laser scans (Figure 23B). Figure 23C shows an overlay of the first and last image of the timescan with Figure 23B pseudocolored blue. 

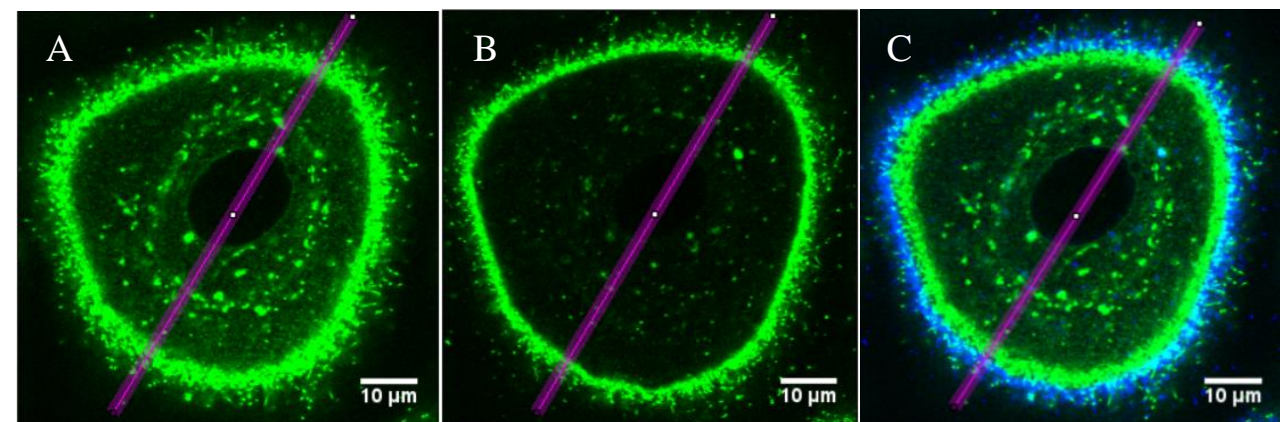

Figure 23: Severe Photobleach of Live FSM Timescans. (A) First image of live timescan. (B)

Last image of live timescan (after 5 minutes with 5 seconds between laser pulses) showing photobleaching, especially evident in the intracellular space. (C) Overlay of first image with pseudo-colored blue last image. The blue signal plasma membrane forms a halo around the green plasma membrane, suggesting the cell is pulling away from the plate and shifting upward in the zaxis.

A profile plot for the purple line in Figure 23 was generated through Image J and reproduced in Figure 24. The green and blue curves represent the first and last image in the time series respectively.

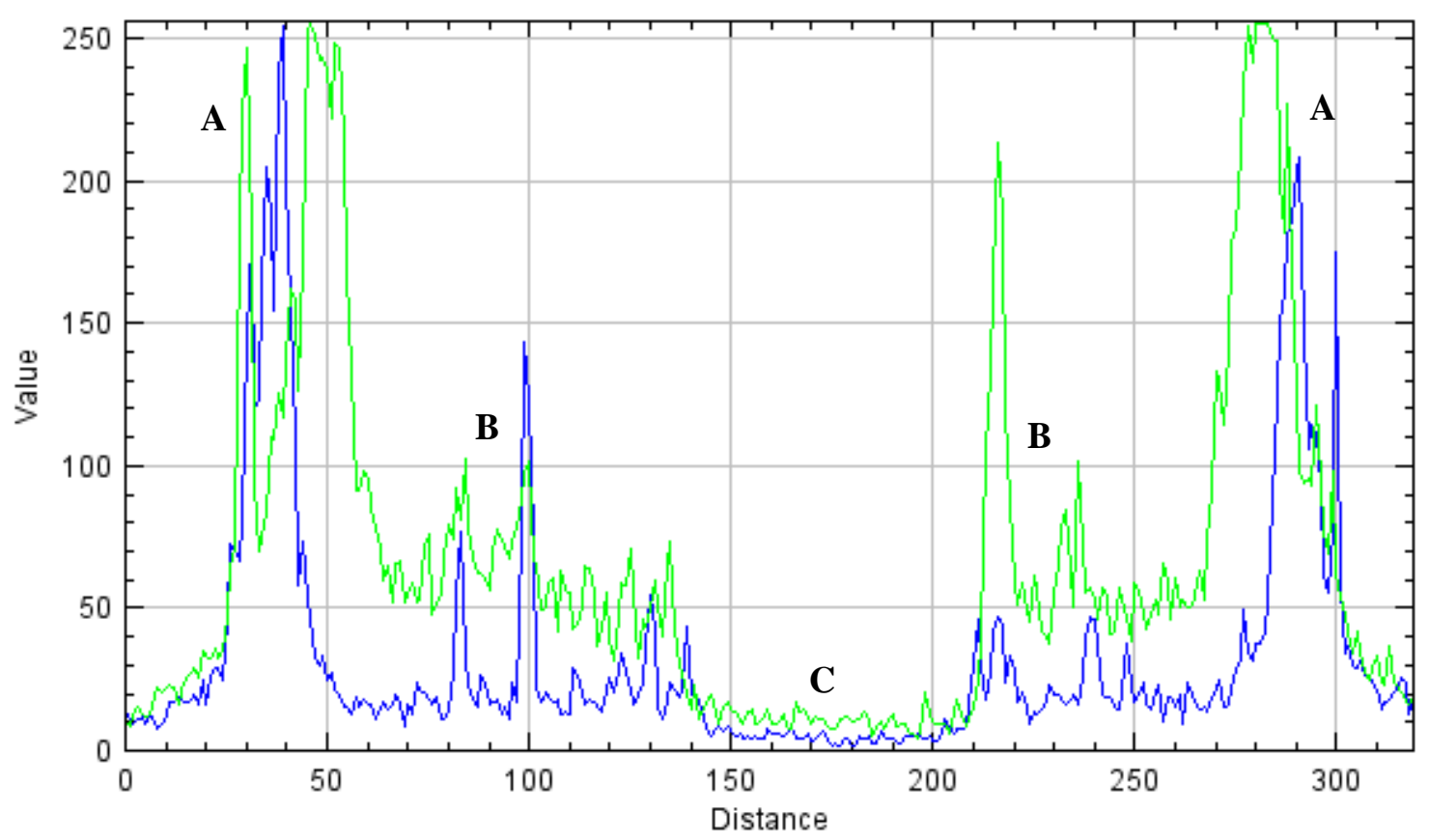

Figure 24: Profile Plot of Signal Degradation and Cell Movement Over Time. The profile plot was constructed from the line drawn across the image in Figure 22. Green represents the first image and blue the last image in the scan. (A) intense plasma membrane localization, (B) intracellular fluorescence clusters near the Golgi elicit small spikes in fluorescence, (C) lack of signal in the nucleus. The blue plot has a significantly lower mean gray value and is shifted outward compared to the green line. 
The intense peaks labeled with "A" in Figure 24 represent the plasma membrane, where two spikes in fluorescence are observed. In between the plasma membrane peaks are smaller peaks as indicated by "B". These correspond to intracellular fluorescence outside the nucleus, possibly in the Golgi. Finally, the drop in fluorescence at " $\mathrm{C}$ " indicates the nucleus, where a lack of fluorescence signal is observed.

Comparing the two curves, the blue exhibited a noticeably lower mean gray value than the green curve, demonstrating the observed photobleaching or fluorescence decrease in signal intensity due to prolonged laser exposure. The two peaks representing the plasma membrane also appeared to shift slightly outward, suggesting movement of the cell during imaging.

\subsubsection{Discussion}

The live trafficking with different continuous $37^{\circ} \mathrm{C}$ incubation times suggested no difference in FSM intensity or localization over time. Both intracellular and plasma membrane staining was apparent even after just 10 minutes of incubation, and the location and relative intensities of the FSM signal during the start of each timescan were comparable. However, after the duration of the imaging, severe photobleaching and slight cell movements were observed. In addition, the plasma membrane fluorescence was brighter with an uneven, coarse FSM staining appearance compared to the previous live imaging experiment, potentially because the FSM solution was not washed off prior to imaging and residual FSM weakly adherent to cells was not removed with washing.

Moving forward, treating the cells with FSM, washing off residual weakly adherent FSM, then fixing the cells with $3.75 \%$ PFA prior to imaging is preferable to live imaging because of the extra variability live imaging exhibits. Although live imaging warrants a more physiologically relevant real-time view of the cell, photobleaching and cell movement can skew data and make image analysis difficult. In addition, the lab was not equipped with a stage heat plate to keep the 
cells at $37^{\circ} \mathrm{C}$ so extended imaging durations could negatively affect cell phenotype. Extended imaging of the same cell culture past 1 hour is not possible with the equipment available in lab. Given the difficulty accounting for photobleaching and cell movement, future work proceeded with a more consistent fixed cell approach. 


\subsection{FSM Temperature Dependence}

\subsubsection{Summary and Objectives}

The goal of the subsequent experiment was to determine if the uptake of FSM is dependent on whether FSM is incubated at $37^{\circ} \mathrm{C}$ prior to imaging. In other words, the goal was to assess if FSM entry into cells is temperature-dependent. It is hypothesized that a $37^{\circ} \mathrm{C}$ incubation with the FSM media facilitates faster FSM incorporation into the cell [59]. In previous experiments with $\mathrm{BSM}$, the lipid was also introduced to cells and incubated at $37^{\circ} \mathrm{C}$ prior to $\mathrm{UV}$ radiation and imaging [13], [14]. If FSM uptake is indeed temperature-dependent for KRT monolayers, ensuring that cells are consistently incubated with FSM at $37^{\circ} \mathrm{C}$ prior to experimentation is necessary in order to reduce extraneous variability. For this experimental protocol, freshly-made FSM was introduced to cells at room temperature and then immediately imaged by taking consecutive time series images. Images were compared to results obtained in previous experiments.

\subsubsection{Results}

Figures 25A-D represent various timepoints within an almost 2-hour imaging session. In the beginning, FSM accumulated in the plasma membrane (Figure 25A-B). Only after about 6090 minutes of live imaging at room temperature did FSM move intracellularly (Figure 25C-D). Furthermore, the intracellular movement was found to be highly heterogeneous and inconsistent, with some cells displaying bright cytoplasmic expression (Figure 25B white arrow). 


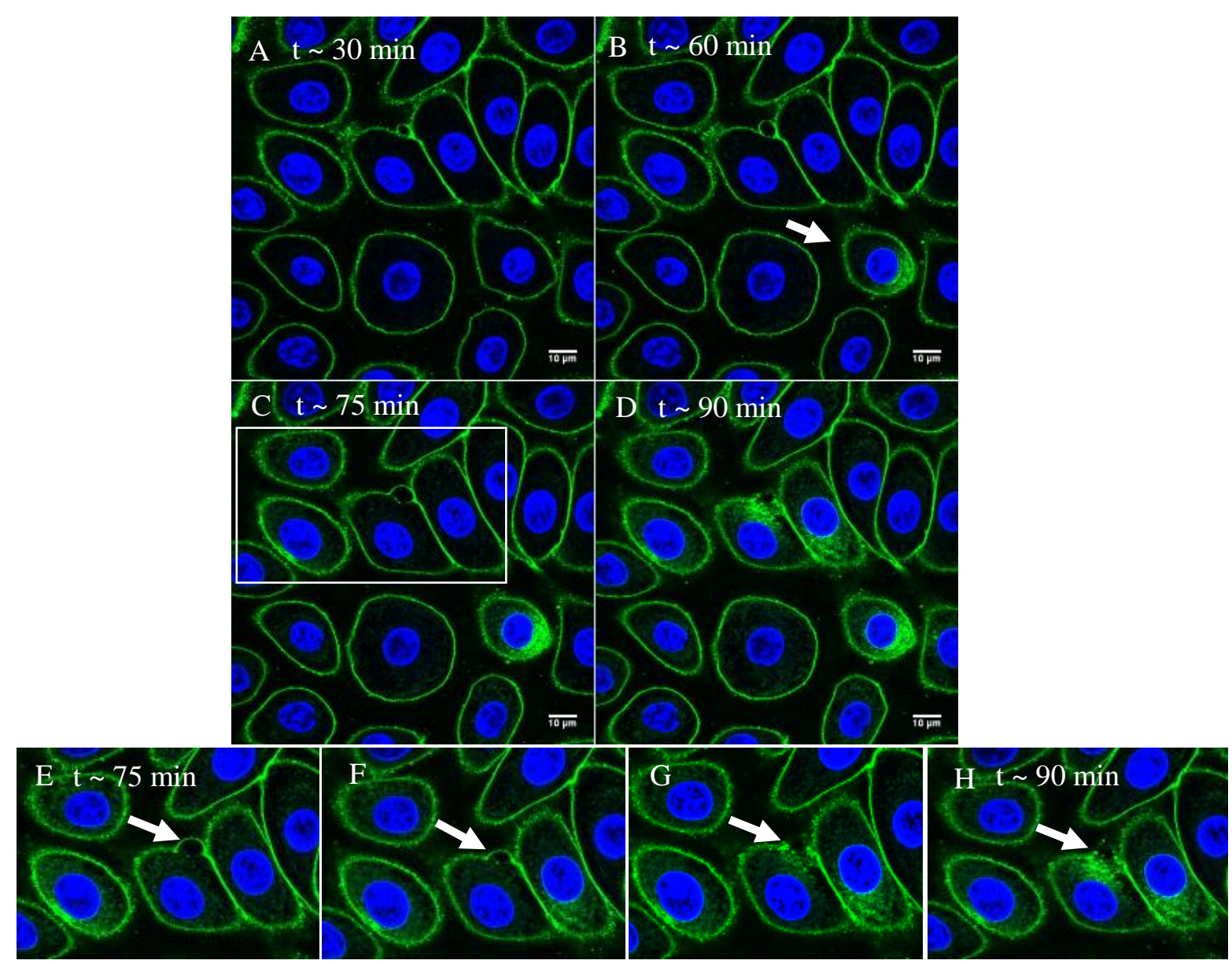

Figure 25: Rapid FSM Entry and Temperature Dependence. (A-D) FSM after approximately

90 minutes (100x objective). Heterogeneous staining is apparent. Some cells start rapidly accumulating FSM, but uptake is highly variable among cells. (E-H) Representative time images between $\mathrm{C}$ and D panel of plasma membrane blebbing and release of FSM into extracellular space after 90 minutes. 100x objective.

Blebbing was again observed, as a cell released FSM to the extracellular space. Figure 25E-H are zoom-in time-lapse images of cells contained within the white box in Figure 25C. As indicated by the white arrows in Figures 25E-H, the cell bleb diminishes in diameter, then eventually releases its fluorescent contents into the extracellular space, most apparent in Figures 25G-H. 


\subsubsection{Discussion}

The previously described experiment suggests that FSM entry and intracellular staining does indeed depend on the temperature. Even after 60-90 minutes of imaging, cytoplasmic staining was inconsistent and heterogenous within the Figure 25 ROI. Some cells displayed bright intracellular fluorescence while others did not. The images suggest endocytic and exocytic blebbing processes are conserved, but perhaps occur at a slower rate because the cells were not cultured at physiologically relevant temperatures $\left(37^{\circ} \mathrm{C}\right)$. Incubation with FSM prior to imaging seems to ensure consistent, homogenous fluorescent labeling of the cells. This experiment suggests that the temperature of FSM incubation is one, of potentially many variables, that need to be conserved in subsequent experiments to reduce variability. It also serves to explain the behavior of BSM which could not be assessed in past research. In the future, all experiments will involve FSM incubation at $37^{\circ} \mathrm{C}$. 


\subsection{Fixed FSM Trafficking with Variable Incubation Times}

\subsubsection{Summary and Objectives}

The goal of this experiment was to quantitively determine if FSM localization in a cell varied over a spectrum of continuous incubation times (10 minutes, 30 minutes, 1 hour, 2 hour, 3 hour, 4 hour, 12 hour, 24 hour). Previous live images suggested that the distribution did not fluctuate significantly, but more consistent methods need to be utilized to yield data worthy of analysis (i.e. PBS wash to remove residual FSM, image a fixed cell culture). In addition, a larger sample size and image analysis program to quantify fluorescence are both necessary to make a conclusion. The results of this experiment are important because fluorescence distribution analysis could point towards an optimal incubation time for the SM treatments.

Eight 8-well plates with monolayers of KRTs were independently incubated with FSM at $37^{\circ} \mathrm{C}$ for varying timepoints. Immediately after treatment, cells were gently washed once with PBS then fixed with 3.75\% PFA. Six wells $(n=6)$ per treatment (3 images/well) were imaged on the confocal using identical laser settings. 2D images were post-processed in ImageJ using the trafficking and rotation ImageJ and MATLAB programs to obtain the mean gray values over normalized distances from the nucleus. Finally, the area under each curve was calculated using MATLAB and plotted. ANOVA and Tukey HSD post-hoc tests were performed on the area data.

\subsubsection{Results}

Figure 26 includes representative images of fixed images during variable FSM incubations. Figure 26A-D show representative images for 10 minutes, 1 hour, 12 hour, and 24 hour, respectively. Throughout all incubation times, the signal intensity and localization appeared to be conserved. Figure $26 \mathrm{E}$ and $\mathrm{F}$ includes a profile plot of a line drawn across the boxed cell in Figure 26A. The plot demonstrates that a brighter intracellular signal (red arrow) and dimmer plasma membrane signal (black arrow) are present in most cell images. 

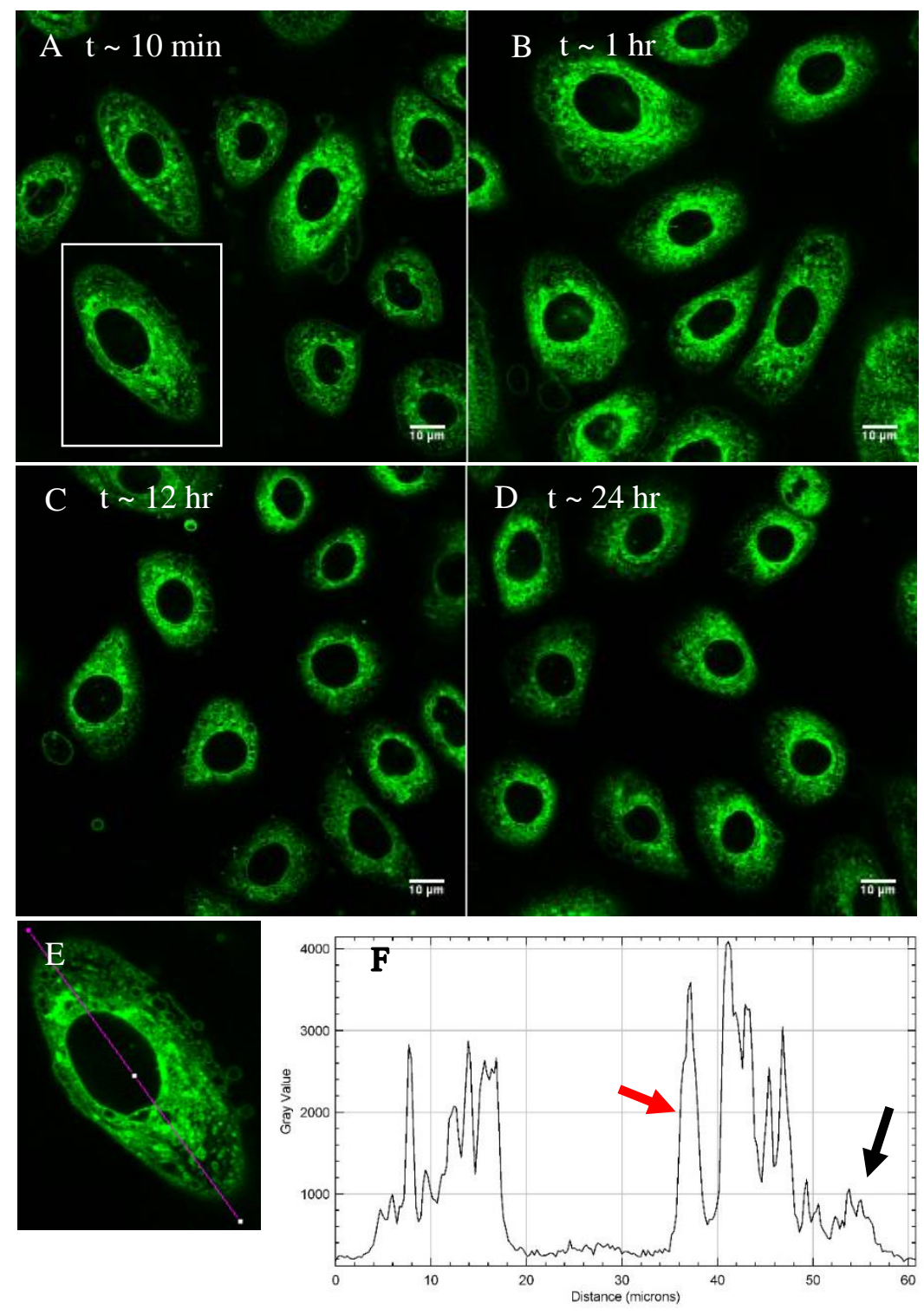

Figure 26: Fixed FSM Trafficking with Variable Incubation Times. (A) $10 \mathrm{~min}$, (B) 1 hour, (C) 12 hour, (D) 24 hour, (E) Profile plot line draw across the boxed cell for the 10 minute treatment. (F) Corresponding profile plot. Red and black arrows denote intracellular and plasma membrane fluorescence respectively. Fluorescence localization and brightness appears to remain consistent regardless of incubation time. 100x objective.

To quantify intracellular fluorescence distribution, the 2D Fluorescence Distribution macros were utilized. Briefly, Region of Interests (ROIs) were drawn around each cell to outline the cytoplasm and nucleus. Lines were extended from the center of each cell nucleus and rotated $360^{\circ}$ at $6^{0}$ increments. The profile plots along each line were averaged. Finally, a MATLAB code 
was used to normalize the average profile plot. Figure 27 includes three representative profile plots (Figure 27A-C) and an outlined cell ROI with corresponding profile plot lines extending radially from the nucleus center (Figure 27D).
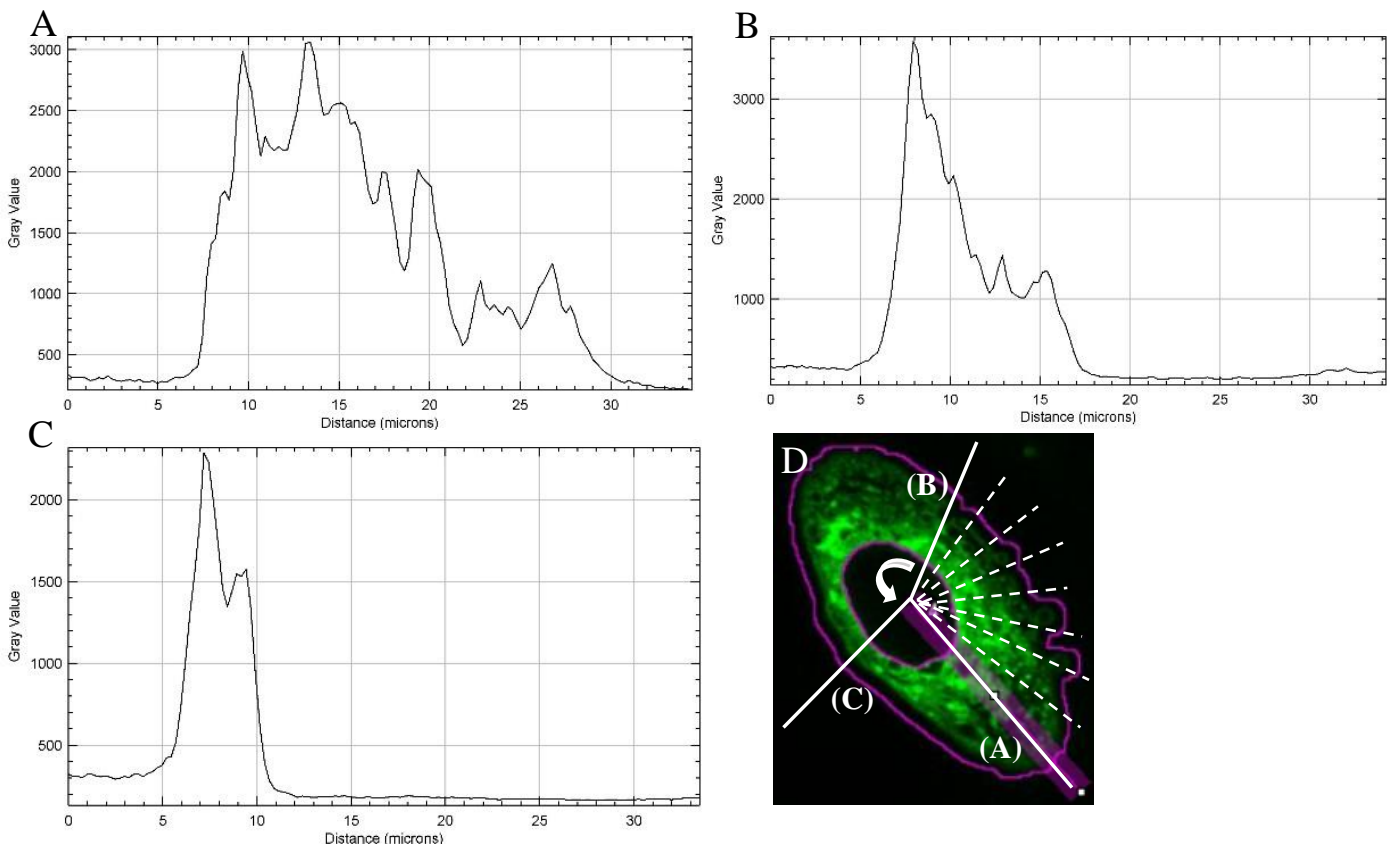

Figure 27: Example Workflow of 2D Fluorescence Distribution. (A-C) Profile Plots corresponding to the lines in (D). For each cell within the image, an ROI was drawn to encompass the cytoplasm and exclude the nucleus (purple outline). Lines extending radially from the centroid were formed and rotated $360^{\circ}$ at $6^{0}$ increments. In total, 60 profile plots per cell were generated. The profile plots along each line were averaged, then the distance was normalized in MATLAB by linear interpolation between points.

The final result of the fluorescence distribution macros was one curve of mean gray value on the $y$-axis and normalized distance from the nucleus on the $\mathrm{x}$-axis. Data from each cell within the image was averaged. Then all three images/well were averaged to obtain one mean gray value vs. normalized distance for each well. Finally, each of the six wells were averaged to yield an average fluorescence intensity curve. Figure 28 includes results of the trafficking data analysis for all eight continuous incubation times (10 minutes, 30 minutes, 1 hour, 2 hour, 3 hour, 4 hour, 12 hour, 24 hour). 


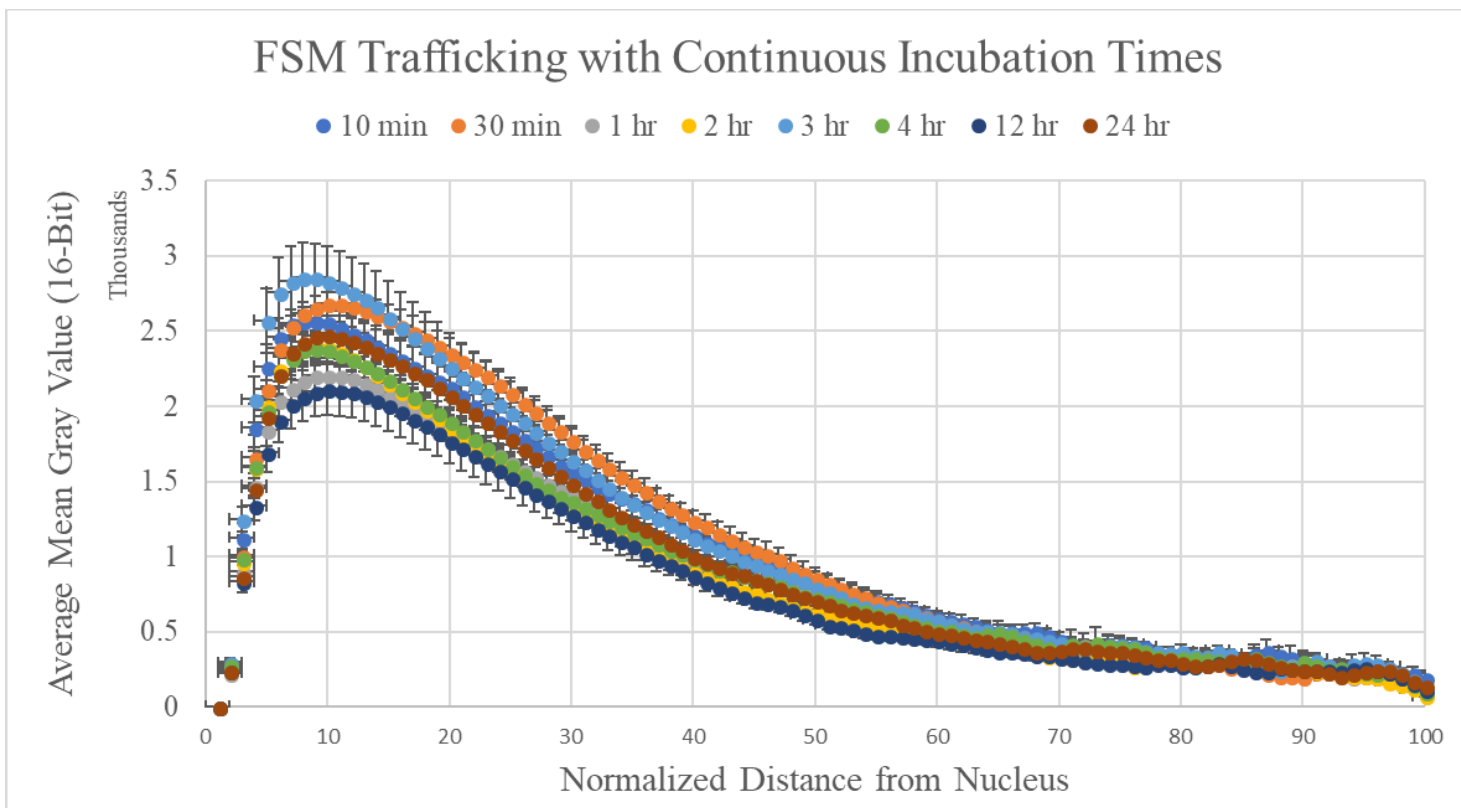

Figure 28: FSM Trafficking with Different Continuous Incubation Times (n=6). Standard error bars are represented.

Over time, the mean gray values slightly fluctuated close to the nucleus, but the variation and intensity considerably diminished farther from the nucleus. The results were supported by previous visual assessment (Figure 26). Independent of the incubation time, the signal was bright around the nucleus, and decreased in intensity farther from the nucleus.

The amount of intracellular FSM corresponding to each treatment was also quantified by calculating the area under each curve with the CUM TRAPZ function in MATLAB. This was done to verify that FSM did not fluctuate significantly over various incubation times. It was expected that curves with a similar shape would yield similar area-under-the-curve measurements. Figure 29 includes a graphical representation of the area calculations. The relative amount of cytoplasmic fluorescence is plotted over 1 hour, 2 hour, 3 hour, 4 hour, 12 hour, and 24 hour FSM continuous incubations. 


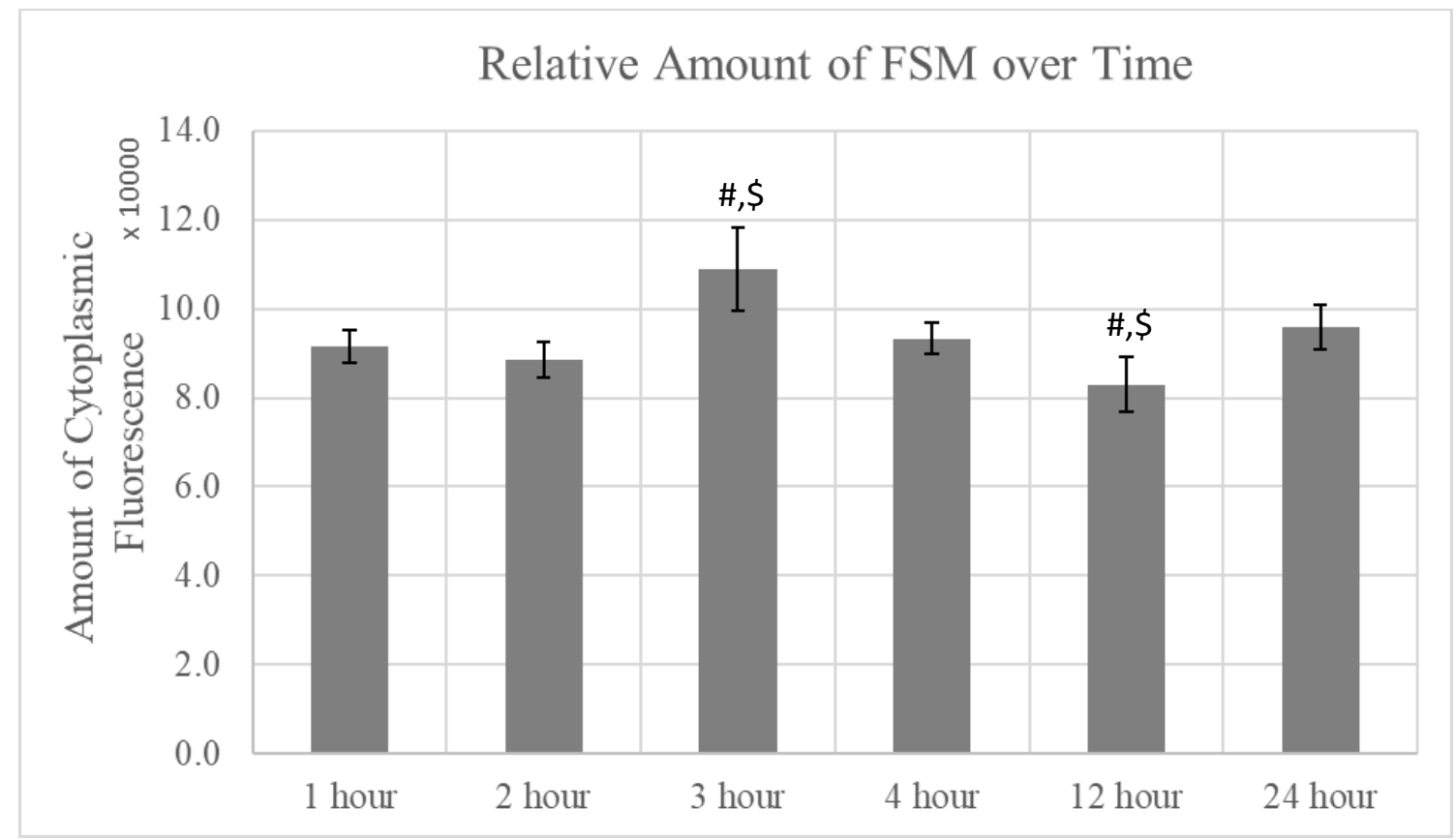

Figure 29: Relative Amount of FSM in a Cell over Various Timepoints (n=6). \# is $p<0.05$ for pairwise comparisons. $\$$ is $p<0.05$ compared to global mean. No other significant pairwise differences between the groups according to Tukey HSD. Standard error bars are represented.

The statistical assumptions were met. Data was normally distributed with equal variances (Appendix B). Furthermore, since each datapoint represented an average of all images within six different wells on a plate, independence was assumed. The 3 hour treatment (M 100.8k, SE 9.2k summative mean gray value) was statistically greater (16.6\%) than the global mean (M 96.7k, SE $5.28 \mathrm{k}$ summative mean gray value) with $\mathrm{p}$-value of 0.0050 . The 12 hour average (M $82.93 \mathrm{k}, \mathrm{SE}$ $6.09 \mathrm{k}$ summative mean gray value) was statistically lower $(11.3 \%)$ than the global mean with pvalue of 0.0413 . Tukey HSD p-value correction was performed on the data and an ordered differences report was generated to perform pairwise comparisons among the timepoints. The data demonstrated that no significant pairwise differences existed when comparing each timepoint to the other except for 3 hour versus 12 hour. Specifically, the average relative amount of fluorescence for 12 hours was $23.9 \%$ lower than the average relative amount for 3 hours (p $0.0279)$. 


\subsubsection{Discussion}

Since data matched observed images, the trafficking and rotation program proved to be a useful analysis technique for the experimental data. The fluorescence distribution in a cell did not change significantly among the timepoints. Although the relative amount of FSM differed between 3 hours and 12 hours, the biological mechanism or significance is unknown. Since this was the only pairwise difference observed in the entire dataset, the difference was deemed to be an artifact of imaging error rather than biological significance. The amount of FSM added is finite and a cell cannot spontaneously generate more intracellular FSM at 3 hours, as the data possibly indicates. For example, fluctuations in laser performance between imaging sessions or small differences in FSM media volume introduced to the cells could induce slight deviations in FSM cytoplasmic fluorescence brightness.

The data suggests that with a continuous supply of FSM in the media, an equilibrium is reached and the cell endocytoses and recycles FSM back to the plasma membrane for exocytosis at an equal rate. Intracellular fluorescence occurs even after just 10 minutes of $37^{\circ} \mathrm{C}$ incubation. The distribution, brightness, and amount of intracellular FSM is also independent of continuous incubation duration. Although contrary to the preliminary hypothesis (Section 2.15), these experimental results suggest that a shorter timepoint for SM incubation prior to UV could be sufficient (i.e. possibly a 1 hour incubation instead of 24 hours). The previous 24 hour incubation was chosen based on literature and prior to FSM adoption [13], [14]. Incubating for one hour can decrease experiment time enabling more convenient and efficient experiments.

Limitations of this experiment include potential error and bias introduced by the analysis program (Figure 30). For example, it was difficult for the program to distinguish surrounding bubbles or blebs from the cytoplasmic outline. 

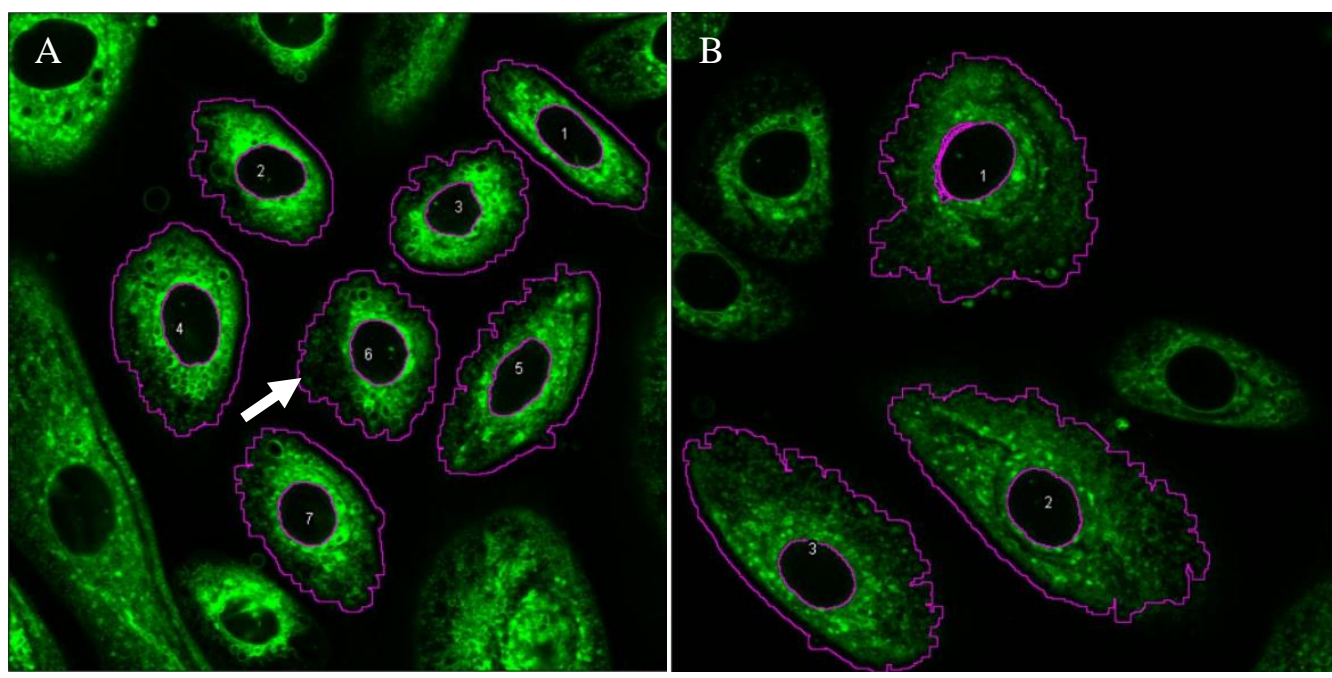

Figure 30: Troubling ROIs. (A) Inaccurate cellular outlines encompassing localized bubbles and blebs (white arrow). (B) Potential cell area data bias. 100x objective.

Resulting protrusions in the outline (Figure 30A white arrow) can potentially skew data since all fluorescence within the ROI is quantified. Furthermore, a large cell versus small cell area potentially introduces data bias that could affect data. Cells with a larger area (Figure 30B) have more cytoplasm and more space between the concentrated Golgi fluorescence and plasma membrane, thus changing the fluorescence gradient and line profile plots. Although data normalization attempts to account for differences in cell size, the full extent of the cell area contribution to the data is unknown. In the future, accounting for cell size would be desirable to determine if a significant interaction exists between fluorescence distribution and cell area.

Finally, the analysis program excludes multi-nucleated cells, and cells along the edge of the image field of view. Furthermore, the program has difficulty outlining out-of-focus images. Improvements to the actual program and implementation for 3D image sets would prove advantageous for the future. 


\subsection{FSM Trafficking with UV}

\subsubsection{Summary and Objectives}

The next step in the progression of goal 1 was to introduce UV to the system. The main goals of the following experiments were to determine if a $40 \mathrm{~mJ} / \mathrm{cm}^{2} \mathrm{UV}$ radiation to a monolayer perturbs intracellular FSM fluorescence distribution and relative amount. Based on a search of recent literature, ceramide levels have been shown to increase after UVB radiation in KRT skin cells which offers photoprotection by increasing the apoptosis rates of potentially damaged cells [24]-[26]. However, the intracellular levels of SM in an irradiated cell are less characterized. Currently, no research groups combine exogenous SM treatment with UVB radiation in KRTs to quantify intracellular SM levels. Wanner et al. added a bolus of $5 \mu \mathrm{M}$ exogenous FSM to KRT cells and used High Performance Thin Layer Chromatography (HPTLC) to demonstrate a 25\% decrease in SM levels to ceramide after 3 hours because of increased hydrolysis by SMase as the cell reaches homeostasis [85]. No UV was introduced to the system. Furthermore, Charruyer et al. and Uchida et al. claimed that ceramide levels increased, but SM levels surprising remained unchanged and did not fluctuate after UV [24], [25]. The ceramide increase was more likely due to CERT dysfunction after UV and was independent of SM levels [24]. However, exogenous SM was not administered, and endogenous levels were only quantified with HPTLC. Additionally, a fluorescence imaging methodology to visualize cellular fluorescence was not employed.

Despite the recent studies, it is unknown whether the addition of both exogenous FSM and UV perturb lipid levels in a significant manner in KRT cells based on fluorescence intracellular distribution analysis. Based on previous research, it was hypothesized that FSM levels would either clearly decrease compared to the no UV control (as FSM is metabolized to ceramide) or remain relatively stable compared to the no UV control (because other mechanisms account for the increase in ceramide). However, the fate of the fluorescent probe is unknown. In 
this current experiment, cells were introduced to FSM for 1 hour or 24 hour continuous incubations, FSM was removed, media was exchanged, and cells were immediately subjected to $40 \mathrm{~mJ} / \mathrm{cm}^{2}$ of UVB radiation. Cells were fixed 1 hour, 2 hour, 12 hour, and 24 hour post-UV. Three confocal images were taken of each well for a total of 6 wells $(n=6)$ in the 1 hour incubation case and 4 wells $(n=4)$ in the 24 hour incubation case. For the control, exact conditions were replicated for the 24 hour plate, except no UV was administered. The role of the control was to delineate metabolism from UV effects when explaining sources of observed FSM fluctuations in cells.

\subsubsection{Results}

Figures 31 and 32 include results of the post-UV fluorescence distributions for a 1 hour and 24 hour continuous incubation. Within each FSM incubation treatment, cells were fixed 1 hour, 2 hours, 12 hours, and 24 hours post-UV and imaged on the confocal. 2D fluorescence distribution analysis was conducted on 3 images per well for a total of 6 wells and 4 wells in the 1 hour and 24 hour incubation treatments respectively. Within each incubation time, the shape of the distribution curve remained consistent, with a spike around the nucleus and decreasing signal approaching the plasma membrane. 


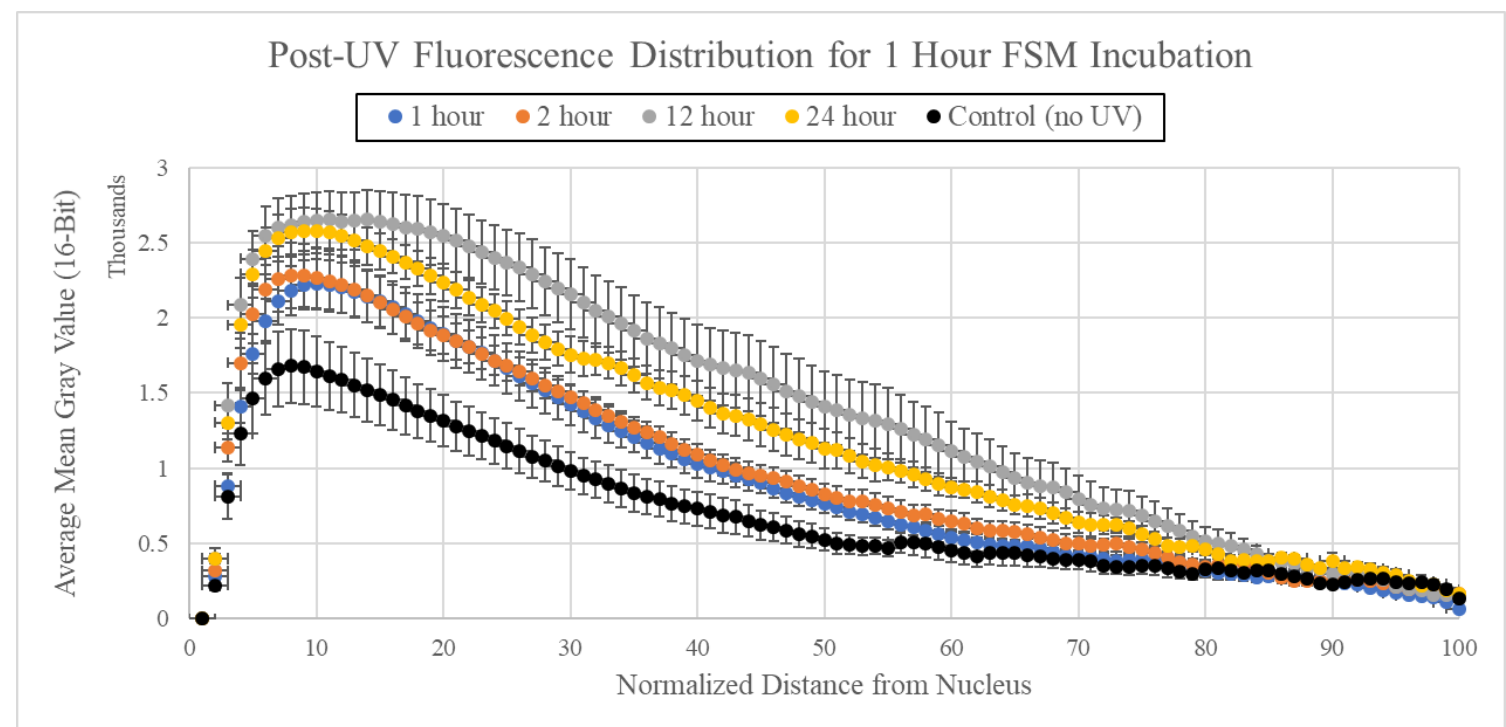

Figure 31: Fluorescence Distribution for 1 Hour Incubation (n=6 wells). Control (no UV) for the 24 hour timepoint is denoted in black. All post-UV timepoints exhibit similar mean gray values and are grouped close together, with the exception of the no UV control with demonstrates a visible reduction in average mean gray value around the nucleus and throughout the cell.

Standard error bars are represented.

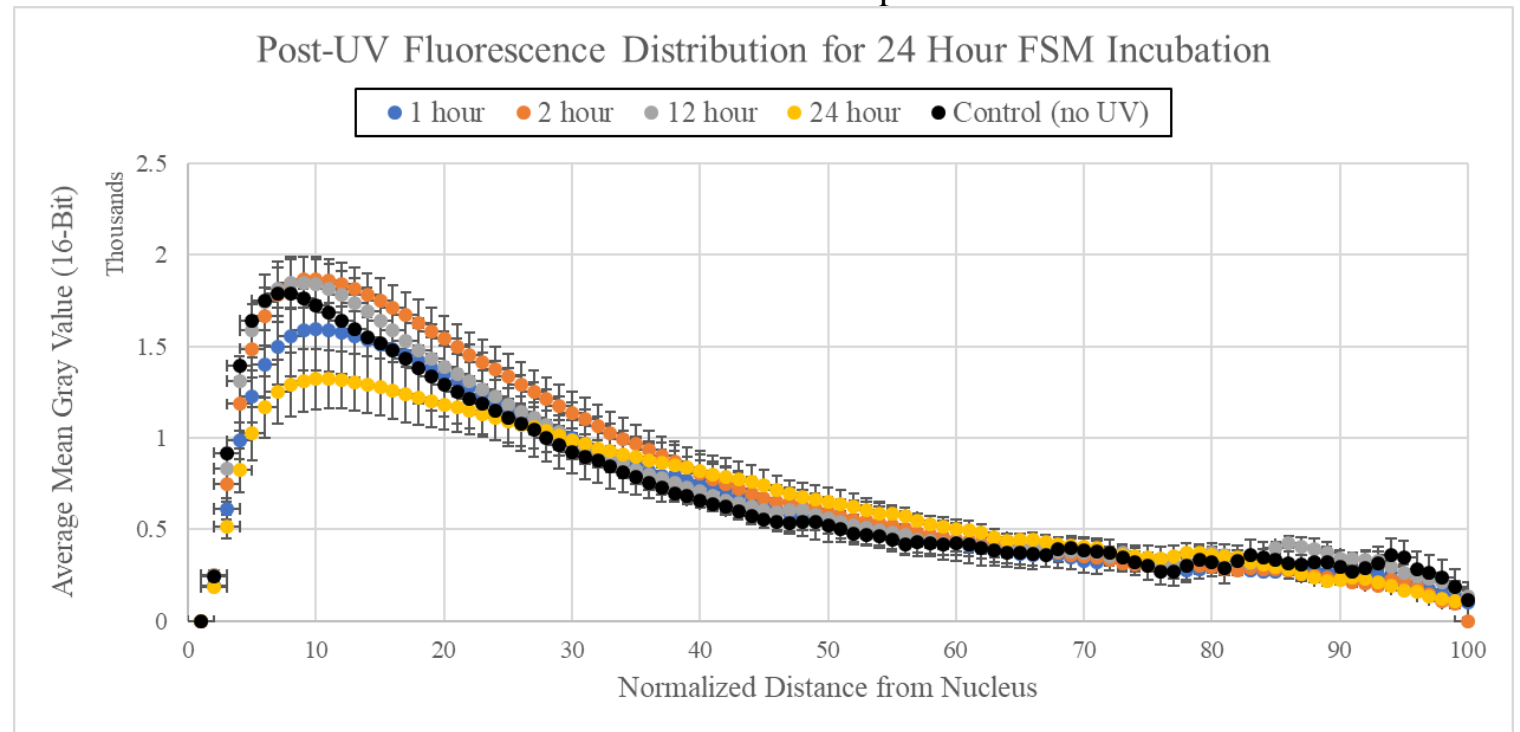

Figure 32: Fluorescence Distribution for 24 Hour Incubation ( $n=4$ wells). Control (no UV) for the 24 hour timepoint is denoted in black. All post-UV timepoints have similar mean gray values throughout the cell and are grouped close together including the no UV control. Standard error bars are represented.

For the 1 hour FSM incubation, the 12 hour curve exhibited a more gradual decline in FSM with higher mean gray values. Additionally, the no UV control (Figure 31 black curve) had a lower mean gray value throughout the cell. All post-UV timepoints within the 24 hour FSM 
incubation including the no UV control (Figure 32 black curve) demonstrated similar mean gray values and curves were grouped close together.

When comparing 1 hour and 24 hour incubation after various post-UV fixation times, one trend was apparent (Figure 33). In the 1 hour, 2 hour, 12 hour, and 24 hour post-UV conditions, the 1 hour FSM incubation time (indicated by the black curve) consistently exhibited a larger mean gray value compared to the 24 hour FSM incubation (gray curve).
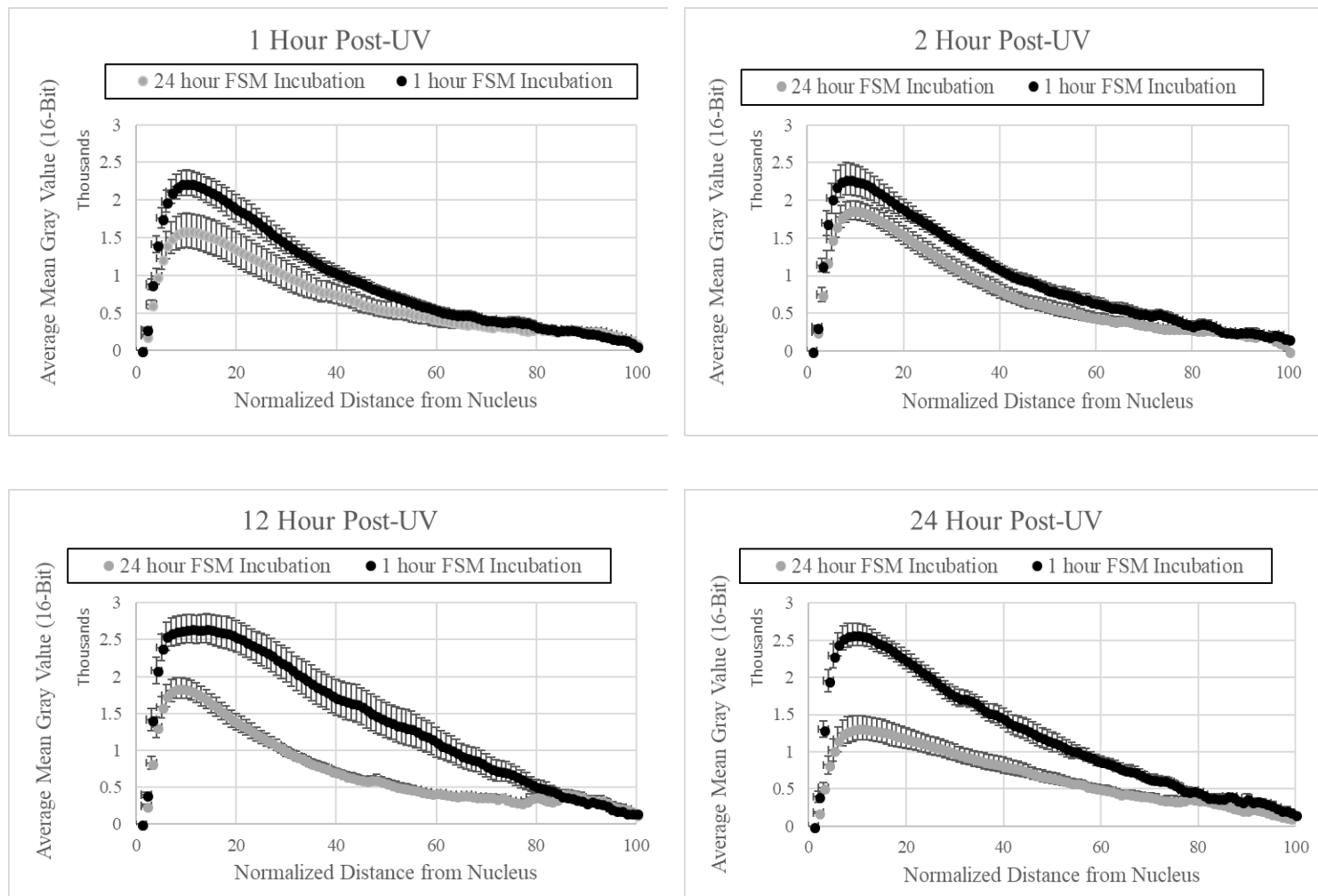

Figure 33: Comparison of Post-UV Fluorescence Distributions for 1 Hour and 24 Hour FSM Incubation. The 1 hour FSM incubation (black curve) had a consistently larger mean gray value compared to 24 hour FSM incubation (gray curve) along the entire cell distance from the nucleus. Standard error bars are represented.

Finally, to confirm the trends demonstrated by the fluorescence distribution graphs, and to provide numbers for statistical data analysis comparisons, the area under each curve was quantified and plotted in Figure 34. Data was normally distributed with equal variances (Appendix B). 


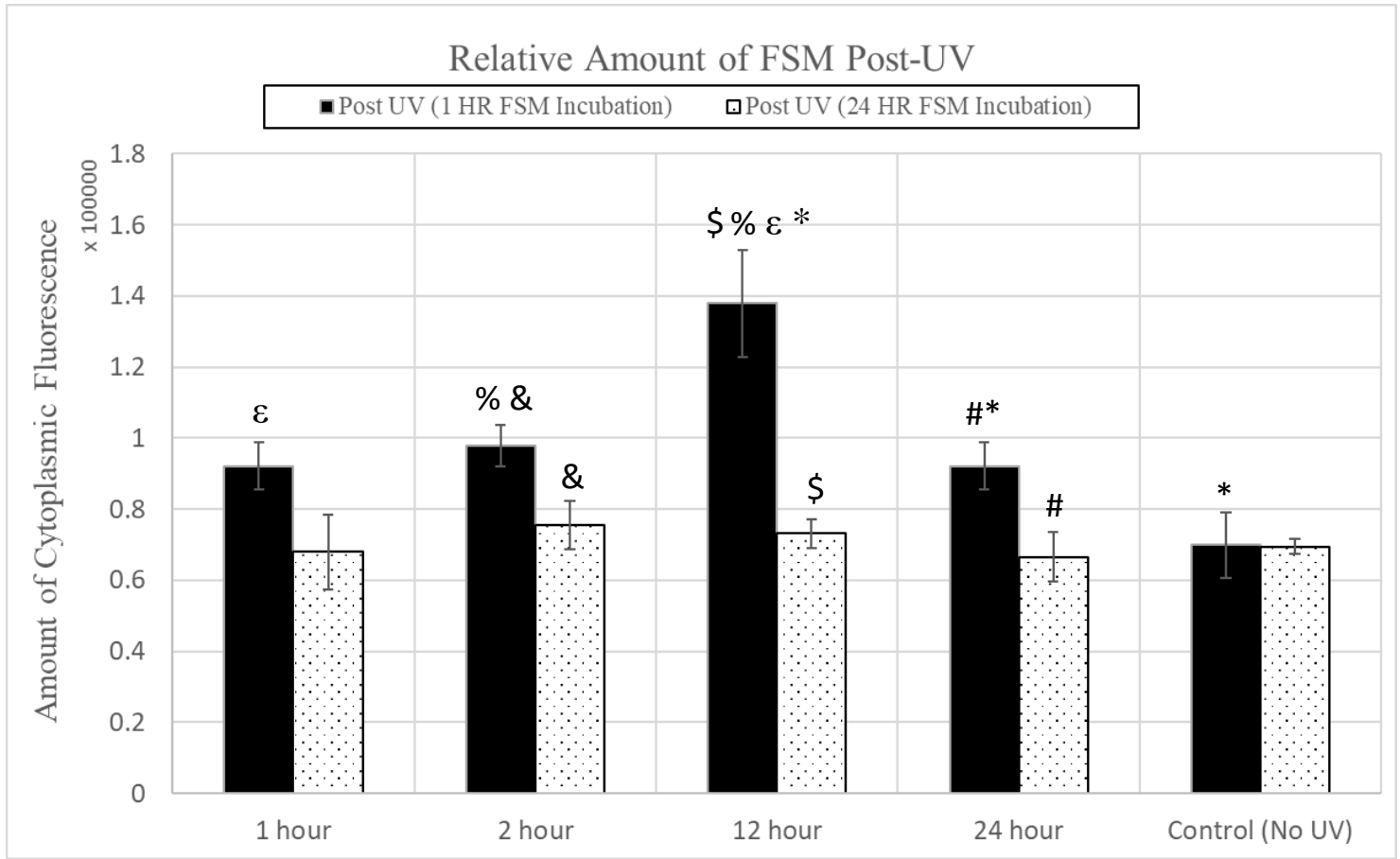

Figure 34: Relative Amount of Cytoplasmic Fluorescence for Various Times after UV.\#, \$, $\%, \varepsilon, *, \&$ denote statistically different comparisons between the two with the same symbol $(\mathrm{p}<0.05)$. Standard error bars are represented.

Tukey HSD pairwise comparisons were conducted in two ways: 1) within the same FSM incubation time (either 1 hour or 24 hour) to compare among post-UV timepoints, 2) within each post-UV timepoint (either 1 hour, 2 hours, 12 hours, or 24 hours) to compare between FSM incubation times. Within the 24 hour FSM incubation treatment, no significant pairwise differences in average cytoplasmic FSM fluorescence were observed among all post-UV timepoints and the no UV control. However, within the 1 hour FSM incubation treatment, some differences in FSM cytoplasmic fluorescence was observed. In particular, the 12 hour post-UV area under the curve (M 137.95k, SE 15k summative mean gray value) was significantly greater than 1 hour (M 92.1k, SE 6.55k summative mean gray values) (p 0.009) and 2 hour (M 97.85k, SE 5.92k summative mean gray values) (p 0.0331 ) by approximately $50 \%$ and $41 \%$ respectively. Furthermore, the no UV control (M 69.96k, SE 9.22k summative mean gray values) was significantly lower compared to the 24 hour post-UV timepoint (M 92.14k, SE 6.55k summative 
mean gray value) (p-value $0.0065,24 \%$ decrease) and 12 hour post-UV timepoint (p-value $0.0002,49.29 \%$ decrease).

Within the same post-UV timepoint, comparisons between the 1 hour and 24 hour FSM incubations were conducted. The 1 hour incubation had a significantly higher average cytoplasmic mean gray value compared to the 24 hour incubation for both the 12 hour (1hour incubation: M 137.95k, SE 15k; 24 hour incubation: M 73.24k, SE 40.3k) (p-value 0.0004, 88\% increase), 2 hour (1 hour incubation: M 97.84k, SE 5.92k, 24 hour incubation: 75.54k, SE 6.86k) (p-value $0.0418,29.53 \%$ decrease), and 24 hour (1hour incubation: M 92.14k, SE 6.55k; 24hour incubation M 66.5k, SE 69.7k) (p-value 0.005, 38.5\% increase) post-UV timepoints.

\subsubsection{Discussion}

Based on trafficking curves and area under the curves, there appears to be an increase in intracellular cytoplasmic cell expression for all post-UV timepoints after just 1 hour of FSM incubation compared to 24 hours FSM incubation. Additionally, FSM levels spiked at 12 hours post-UV for the 1 hour incubation while remaining fairly consistent throughout the 24 hour incubation. Within the 1 hour timepoint, 24 hour post-UV had more fluorescence expression than the control, 1 hour, and 2 hour post-UV timepoints. Lastly, no difference between the no UV controls were observed.

It is unknown at this time why the intracellular fluorescence significantly increased at 12 hours, especially since no new FSM is introduced to the system after UV exposure. Perhaps the increase is artificial and a result of variability or error in the measurement system or experimental parameters. More replicates are needed to further investigate this observation. Also, given the variability in the post-UV timepoints within the 1 hour incubation, more experiments are warranted to further understand the effects of a 1 hour incubation. A 1 hour FSM incubation shows promise because more variability among timepoints was observed, suggesting a 1 hour 
incubation could elicit more biologically relevant changes post-UV. For example, the significant difference between 24 hour post-UV and control for 1 hour FSM incubation might suggest that UV alters cells to stop metabolizing FSM. No significant difference was exhibited between 24 hour post-UV and 1 hour post-UV for the 1 hour FSM incubation. Furthermore, it is unknown why all 1 hour incubation FSM amounts were greater than 24 hour, especially since the previous FSM trafficking experiment demonstrated no significant difference among continuous incubation timepoints. Perhaps a combination of variable FSM metabolism rates and UV perturbation cause a significantly greater signal after 1 hour incubation.

According to the preliminary mechanism hypothesis (Section 2.15), intracellular levels of post-UV FSM were expected to be lower than the no-UV control because FSM is hydrolyzed to ceramide and the fluorescent probe is cleaved and exocytosed from the cell. The exocytosed probe after FSM hydrolysis would result in a diminished total intracellular fluorescence. However, the data demonstrated contrary results (post-UV timepoints had greater intracellular FSM compared to control), highlighting the need to identify exactly where the downstream fluorescent probe localizes in a cell after FSM hydrolysis. Future colocalization experiments with FSM, lysenin (endogeneous SM), and ceramide could help elucidate the identity of the fluorescent signal - whether the fluorescence is an SM (colocalized with lysenin) or a fluorescent ceramide (colocalized with ceramide).

This experiment demonstrated the value of controls. Comparisons cannot be made between UV and no UV for each timepoint because of the experimental conditions. The trafficking experiments involved continuous incubations with FSM. Experimental plates for the UV experiments, on the other hand, were incubated continuously with FSM for 1 hour or 24 hours then the media was removed and non-FSM media was replaced immediately prior to UV. Post-UV, the cells were incubated in the regular KRT media for 1 hour, 2 hours, 12 hours, and 24 hours. Thus, a direct comparison between each trafficking timepoint and the UV timepoint cannot 
be made because two variables - the UV exposure and latent post-UV incubation in clean media prior to fixing - confound the results. The latent incubation without a constant supply of FSM would most likely decrease FSM intensity due to metabolism and decrease the fluorescence intensity. If a change was observed, it is unknown whether the difference would be from UV perturbation or FSM metabolism. The only control performed in this experiment was for the 24 hour timepoints. In the future, controls for each timepoint are necessary to draw more relevant conclusions. 


\section{6 p21 Ratios for BSM and FSM Comparison}

\subsubsection{Summary and Objectives}

The UV photoprotection benefit of BSM was compared to FSM, a fluorescent analogue of BSM, by quantifying the p21 positive to total cells within each well. P21 is an indicator of DNA damaged cells. Past studies have suggested BSM photoprotection for KRTs by demonstrating decreased p21 levels in UV exposed cells after BSM treatment compared to control [13], [14]. However, the functional equivalence of FSM as a suitable analogue for BSM has not been demonstrated. The goal of this experiment was to determine if FSM treatment reduces p21 levels in KRT cells after UV radiation in a similar manner to BSM.

\subsubsection{Results}

Cells were incubated with either BSM or FSM for 24 hours, washed, and exposed to 40 $\mathrm{mJ} / \mathrm{cm}^{2}$ of UV radiation. 24 hours post-UV, cells were fixed and stained for $\mathrm{p} 21$. The ratios of positive p21 to total cells were quantified for each treatment. Figures 35 and 36 include representative images of the SM treatment and control cases for both no UV and UV respectively. 

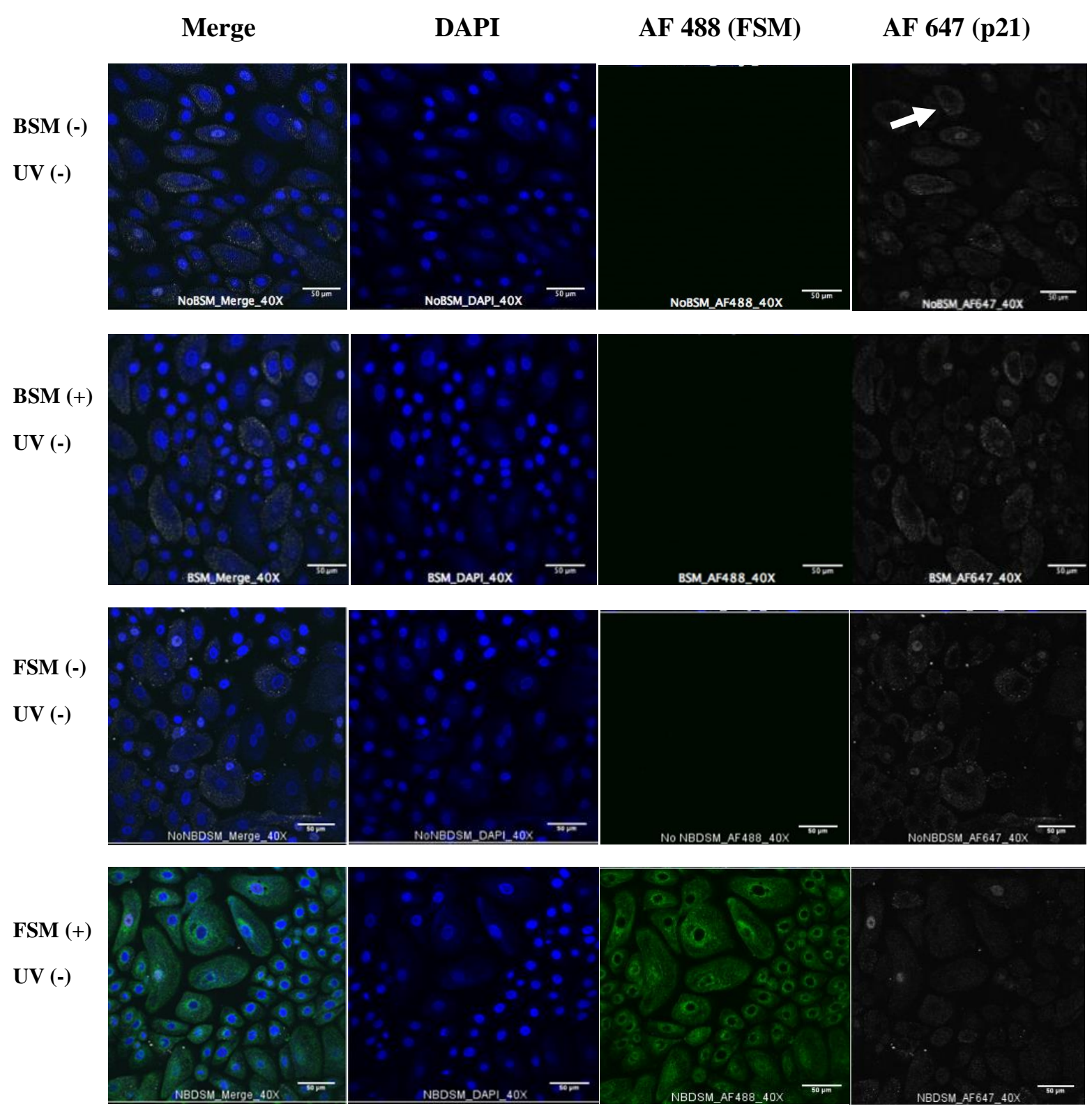

Figure 35: No UV with BSM and FSM Treatment. (Top Row) BSM (-), UV (-). ( $2^{\text {nd }}$ Row) BSM (+), UV (-). (3 ${ }^{\text {rd }}$ Row) FSM (-), UV (-). (Bottom Row) FSM (+), UV (-). Images demonstrate relatively low p21 nuclear expression (white arrow) suggesting that SM incubations are not cytotoxic. 40x objective. 

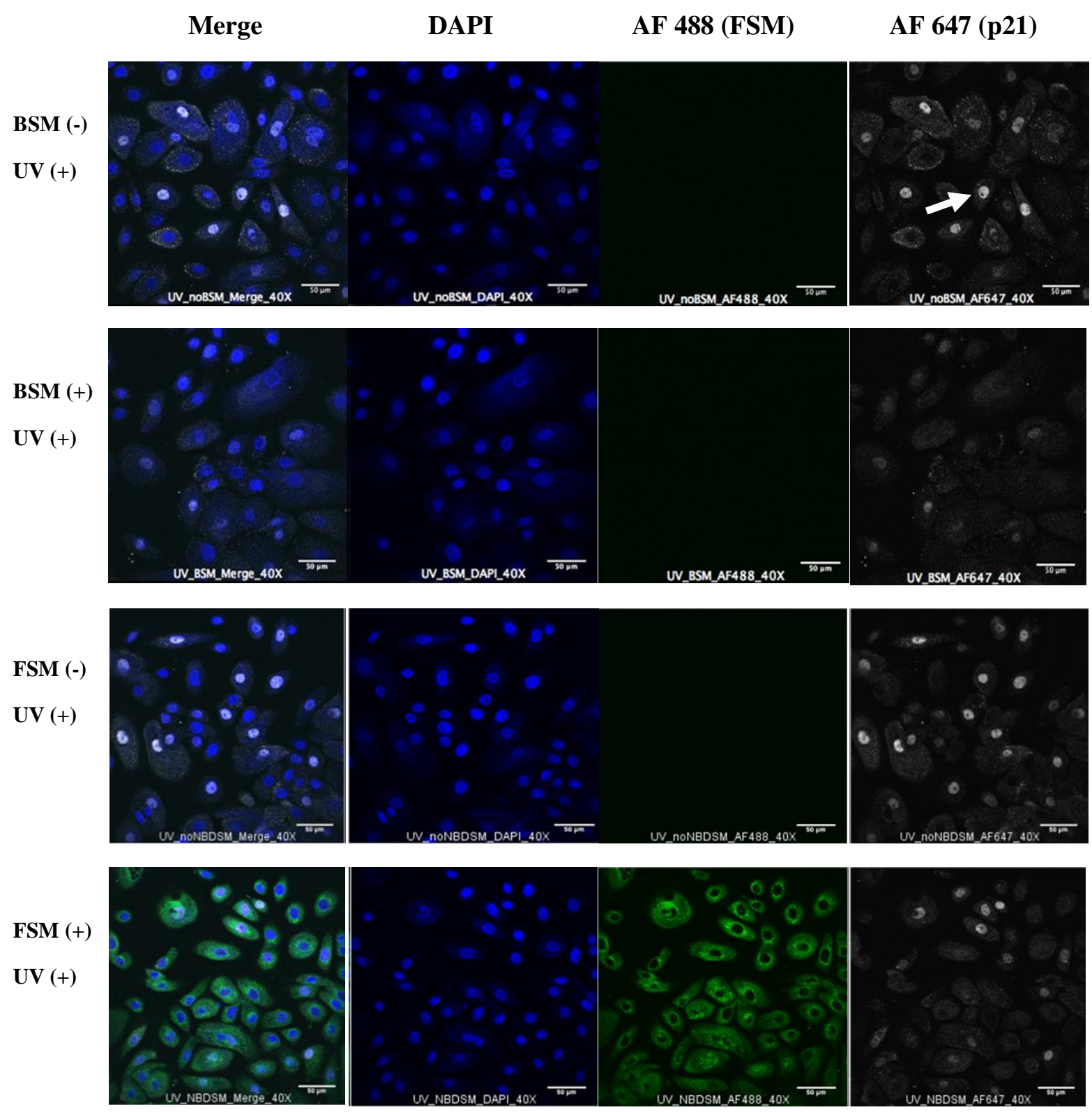

Figure 36: UV with BSM and FSM Treatment. (Top Row) BSM (-), UV (+). ( $2^{\text {nd }}$ Row) BSM $(+)$, UV (+). ( $3^{\text {rd }}$ Row) FSM (-), UV (+). (Bottom Row) FSM (+), UV (+). Reduced amount of nuclear p21 levels (white arrow) are apparent in the SM treatment cases compared to control no SM treatments. 40x objective. 
The above figures are separated by treatment and channel (either merge, DAPI, AF 488, or AF 647). DAPI shows nuclei, AF 488 shows the presence (or absence) of green FSM signal, and AF 647 indicates p21 fluorescence. The white arrow in Figure 35 demonstrates a clear lack of p21 nuclear signal (although faint baseline cytoplasmic expression is observed). Furthermore, the white arrow in Figure 36 denotes clear nuclear p21 expression which is easily distinguished and brighter than the baseline cytoplasmic expression.

Visual comparison of nuclear p21 expression among the images demonstrates that the no UV case has lower p21 expression regardless of SM treatment. The lower p21 levels suggest introducing BSM or FSM does not elicit cytotoxicity or increased cell cycle arrest levels compared to control. When $40 \mathrm{~mJ} / \mathrm{cm}^{2}$ of UVB is introduced to the cells, nuclear p21 expression increases but the number of nuclei stained in the BSM and FSM treatments are lower. This observation supports photoprotective hypotheses for SM. Lower nuclear p21 levels correspond with decreased cell cycle arrest and photoprotection after UV. The observations were confirmed by calculating p21 ratios (p21 positive nuclei divided by total nuclei) for each image (Figure 37). Three confocal images were obtained per well, and four wells were analyzed per treatment $(n=4)$. 

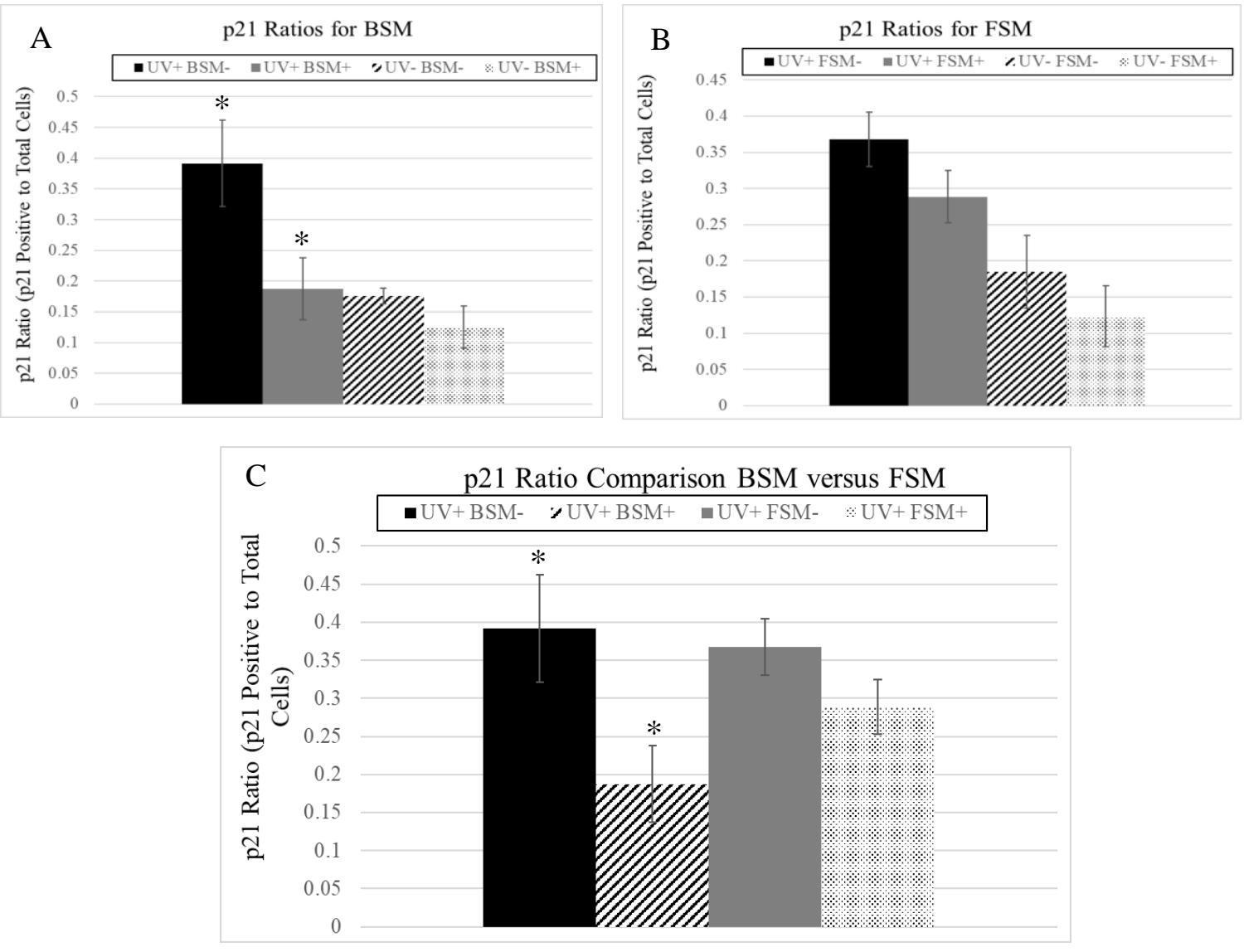

Figure 37: p21 Ratio Results (n=4 wells). (A) BSM p21 Ratios. (B) FSM p21 Ratios. (C) Comparison Between Bovine Milk SM and C6-NBD SM p21 Ratios for UV Cases. Standard error bars are represented.

P21 ratio results displayed photoprotective trends. Appendix B includes normality assumptions. Within the UV cases, the number of positive p21 cells was lower after treatment compared to no treatment for both FSM and BSM. As shown in Table VII, either adding BSM or FSM to UV(-) cells did not increase the p21 ratios. Furthermore, adding BSM and FSM decreased the p21 ratio after UV treatment. BSM and FSM decreased p21 levels by $51 \%$ and $21.6 \%$ respectively. BSM exhibited a significant difference (p-value 0.0004). Although Tukey HSD pairwise comparisons did not indicate significance for FSM because of the low sample size, the trends are promising and would most likely demonstrate statistical significance given more replicates. 
Table VII: Averages for All Treatments Demonstrating Trends

\begin{tabular}{|c|c|c|}
\hline Treatment & $\begin{array}{c}\text { Average (ratio of p21 } \\
\text { positive nuclei to total } \\
\text { nuclei) }\end{array}$ & SE \\
\hline $\begin{array}{c}\text { BSM (-) } \\
\text { UV (-) }\end{array}$ & 0.18 & 0.0125 \\
\hline $\begin{array}{c}\text { BSM (+) } \\
\text { UV (-) }\end{array}$ & 0.13 & 0.0345 \\
\hline $\begin{array}{c}\text { BSM (-) } \\
\text { UV (+) }\end{array}$ & 0.39 & 0.075 \\
\hline $\begin{array}{c}\text { BSM (+) } \\
\text { UV (+) }\end{array}$ & 0.19 & 0.05 \\
\hline $\begin{array}{c}\text { FSM (-) } \\
\text { UV (-) }\end{array}$ & 0.18 & 0.05 \\
\hline $\begin{array}{c}\text { FSM (+) } \\
\text { UV (-) }\end{array}$ & 0.12 & 0.0419 \\
\hline $\begin{array}{c}\text { FSM (-) } \\
\text { UV (+) }\end{array}$ & 0.37 & 0.0373 \\
\hline $\begin{array}{c}\text { FSM (+) } \\
\text { UV (+) }\end{array}$ & 0.29 & 0.0358 \\
\hline
\end{tabular}

\subsubsection{Discussion}

Although no significant differences were observed between FSM treatments, data suggests that FSM provides similar photoprotection to UVB exposed KRTs while not inducing cytotoxic effects. FSM decreased p21 levels by $21.6 \%$ compared to the no FSM control. Similarly, BSM decreased p21 levels by $51 \%$ compared to the control, which was statistically significant.

The lack of significance for FSM and the smaller decrease in p21 ratio could be due to concentration discrepancies between FSM and BSM. Based on the molecular weight of bovine milk SM on the Avanti Polar Lipids website, the $0.1 \%$ BSM is equivalent to a $1.3 \mathrm{mM}$ concentration [61]. Thus, the concentrations of FSM and BSM are not equivalent and the FSM concentration is significantly lower, possibly contributing to the reduced levels in photoprotection compared to BSM. Further research is needed to reconcile the concentration differences and optimize the concentration of FSM to yield maximum levels of p21 photoprotection. 


\subsection{FSM with Nile Red}

\subsubsection{Summary and Objectives}

Nile red was introduced as a general lipid stain with a purpose to co-stain FSM in order to help with characterization. Colocalization of FSM with another stain could potentially help with identification (i.e. if the fluorescent signal is SM, if fluorescent SM has been degraded to ceramide, or if the signal is freely floating fluorescence). Specifically, FSM signal colocalization with Nile red would suggest whether the signal was attached to a general lipid (SM, ceramide, or another metabolite) or just a freely floating probe.

The goal of this experiment was to determine the appropriateness of Nile red as a general lipid stain for colocalization with FSM. FSM was incubated with cells for 1 hour then removed, washed once with PBS, then fixed. $200 \mu \mathrm{L}$ of $5 \mu \mathrm{M} / \mathrm{mL}$ Nile red was introduced to cells for 15 minutes, then washed off. A nuclei counterstain was also performed.

\subsubsection{Results}

As Figure 38 demonstrates, the Nile red stain was robust with a high signal to noise ratio and yielded an intense signal throughout the entire cell. Figures 38A-D represent Nile red and FSM dual staining while Figures 38E-H correspond to cells only stained with Nile red (no exogenous FSM incubation). 

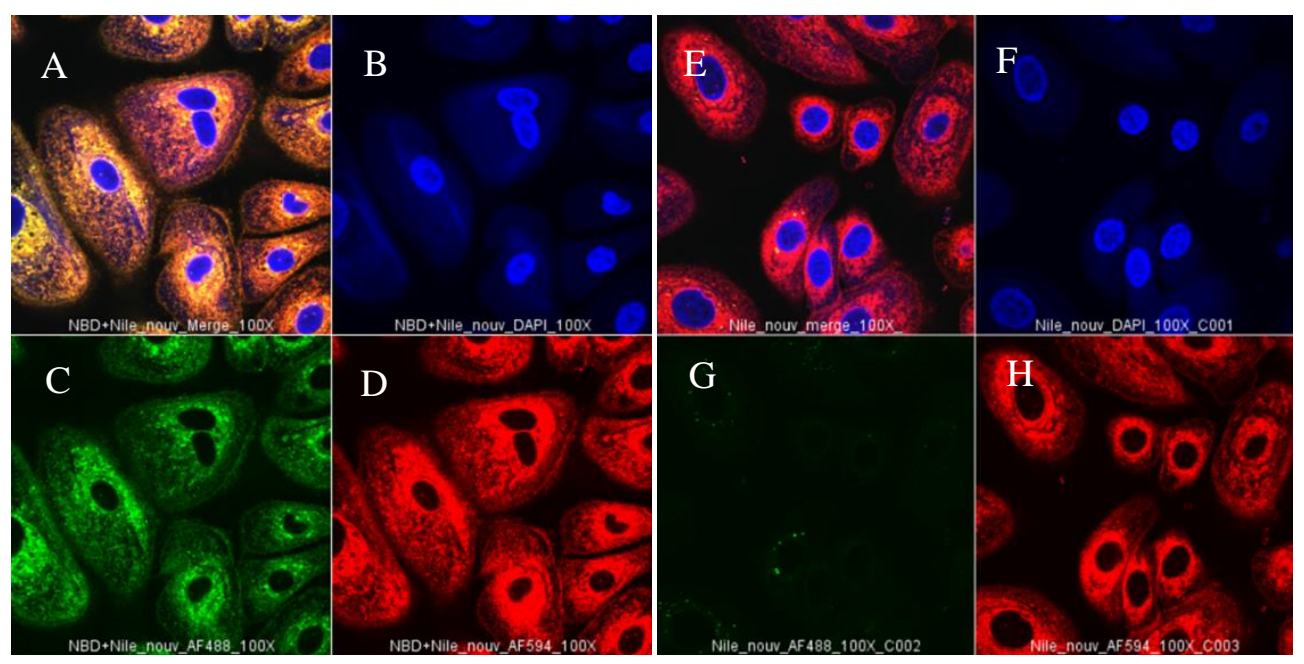

Figure 38: Nile Red Representative Images. A-D Nile Red and FSM Dual Staining (100x objective) (A) Merge of Nile Red, FSM, and Hoechst. (B) Hoechst, (C) FSM, (D) Nile Red. E-H Nile Red Only (100x objective) (E) Merge, (F) Hoechst, (G) AF 488 channel (no FSM), (H) Nile Red. Some bleed through was observed from Nile red into the AF488 channel. With the addition of exogenous FSM, the brightness of the Nile red increased in (D) compared to (H).

Visual comparison between the two Nile red images (Figure 38D and 38H) indicate that the dual stain with exogenous FSM yielded a brighter Nile red general lipid stain. This suggests Nile red was successful in demonstrating the increase in total cellular lipid content that occurs when exogenous FSM is introduced to a cell. However, some slight bleed through was observed in Figure 38G, most likely due to the bright Nile red signal and spectral overlap between the AF 488 and AF 594 filters used to detect FSM and Nile red respectively.

\subsubsection{Discussion}

Nile red proved to be a robust, bright stain with high signal to noise ratio. Furthermore, the Nile red brightness increased after FSM incorporation, suggesting it is sensitive enough to detect an increase in intracellular lipid content. However, the applications for Nile red are limited because it is a general lipid stain and not specific to either ceramide or lysenin. Ideally, a stain that only binds to endogenous SM or ceramide would provide a better way to identify FSM identity with colocalization quantification. Nile red was removed from future protocols, and 
optimization was conducted on two new immunofluorescence stains, lysenin and ceramide, to quantify natural endogenous levels of sphingomyelin and ceramide and assess how exogenous introduction of FSM affects intracellular localization and relative abundance. 


\subsection{Lysenin Stain Optimization}

\subsubsection{Summary and Objectives}

Lysenin is a novel probe that has been recently developed for fluorescence detection of endogenous cellular SM. Lysenin, is a 41-kDa protein obtained from purified coelomic fluid of the earthworm Eisenia foetida that binds selectively to SM [64]. Recent work has used lysenin and FSM to mark plasma membrane lipid rafts [65]. Building off of past research, a lysenin immunofluorescence protocol for this thesis was developed based on concentrations in published literature [64]. Immunofluorescence staining generally involves incubating the sample in the lysenin protein, washing off residual, incubating with a primary antibody against the lysenin protein, washing off residual primary, subsequently introducing a secondary against the species of the primary antibody, then washing and imaging [64] . A blocking buffer is also introduced to prevent non-specific binding of the lysenin protein, primary antibody, and secondary antibody [64].

The goals of these lysenin optimization studies were to identify the optimal lysenin, primary antibody, and secondary antibody in order to reduce non-specific binding while obtaining a high signal to noise ratio. Three experiments were conducted to vary the blocking, lysenin concentration, primary concentration, and stain incubation times. Secondary concentration remained at a consistent concentration and incubation time throughout all experiments. The following results section includes a chart of experimental factors corresponding to each optimization step followed by images.

\subsubsection{Results}

Table VIII includes the experimental variables tested for in the first optimization study. Both the lysenin protein and primary antibody concentrations were varied while incubation time and blocking remained consistent. First, fixed 8-well plates were blocked with $2 \%$ BSA in PBS 
overnight, then lysenin protein (at a concentration of $1 \mu \mathrm{g} / \mathrm{mL}$ or $3 \mu \mathrm{g} / \mathrm{mL}$ ) was incubated with cells for 2 hours. Anti-lysenin primary antibody was subsequently introduced at dilutions of $1 / 1000,1 / 500$, or $1 / 300$ in PBS for 9 hours. Finally, a secondary antibody was added at a 1/300 concentration in PBS for 1 hour and a Hoechst counterstain was performed. Thorough washing steps with PBS were added between each staining step. Table VIII also indicates the controls performed in the study, to assess whether non-specific binding or bleed through is a common occurrence. In the table, " $\mathrm{X}$ " denotes the indicated procedure was performed and "N/A" means that particular step was skipped.

Table VIII: Optimization 1: Pilot Study

\begin{tabular}{|c|c|c|c|c|c|c|}
\hline \multicolumn{3}{|c|}{ IF Stain } & \multicolumn{2}{|c|}{ Concentration } & \multicolumn{2}{|c|}{ Incubation Time } \\
\hline \multicolumn{3}{|c|}{ Blocking (B) } & \multicolumn{2}{|c|}{$2 \%$ BSA in PBS } & \multicolumn{2}{|c|}{ Overnight } \\
\hline \multicolumn{3}{|c|}{ Lysenin (L) } & \multicolumn{2}{|c|}{$1 \mu \mathrm{g} / \mathrm{mL}$ or $3 \mu \mathrm{g} / \mathrm{mL}$} & \multicolumn{2}{|c|}{$2 \mathrm{hr}$} \\
\hline \multicolumn{3}{|c|}{ Anti-Lysenin Primary Ab (1) } & \multicolumn{2}{|c|}{$1 / 1000,1 / 500,1 / 300$} & \multicolumn{2}{|c|}{$9 \mathrm{hr}$} \\
\hline \multicolumn{3}{|c|}{ Secondary $(2)$} & \multicolumn{2}{|c|}{$1 / 300$} & \multicolumn{2}{|c|}{$1 \mathrm{hr}$} \\
\hline \multicolumn{3}{|c|}{ Hoechst $(\mathrm{H})$} & \multicolumn{2}{|r|}{$0.05 \%$} & \multicolumn{2}{|c|}{$15 \min$} \\
\hline Control & Blocking & Lys & & Primary & Secondary & Hoechst \\
\hline $\mathrm{H}$ & $\mathrm{X}$ & $\mathrm{N}$ & & N/A & $\mathrm{N} / \mathrm{A}$ & $\mathrm{X}$ \\
\hline $\mathrm{H}+1$ & $\mathrm{X}$ & $\mathrm{N}$ & & $\mathrm{X}(1 / 300,9 \mathrm{hr})$ & $\mathrm{X}(1 / 300,1 \mathrm{hr})$ & $\mathrm{X}$ \\
\hline $\mathrm{H}+1+2$ & $\mathrm{X}$ & $\mathrm{N}$ & & $\mathrm{X}(1 / 300,9 \mathrm{hr})$ & $\mathrm{X}(1 / 300,1 \mathrm{hr})$ & $\mathrm{X}$ \\
\hline $\mathrm{H}+\mathrm{L}+1$ & $\mathrm{X}$ & $\mathrm{X}(1 \mu \mathrm{g} /$ & & $\mathrm{X}(1 / 300,9 \mathrm{hr})$ & N/A & $\mathrm{X}$ \\
\hline $\mathrm{H}+\mathrm{L}+2$ & $X$ & $\mathrm{X}(1 \mu \mathrm{g} /$ & & N/A & $\mathrm{X}(1 / 300,1 \mathrm{hr})$ & $X$ \\
\hline
\end{tabular}

Controls ensure that the fluorescence observed in the experimental condition $(\mathrm{H}+\mathrm{L}+1+2)$ is truly an indicator of endogenous sphingomyelin levels and not a result of random binding or fluorescence by lysenin, primary, or secondary antibodies. The roles of the $\mathrm{H}, \mathrm{H}+1$, and $\mathrm{H}+\mathrm{L}+1$ controls were to confirm that Hoechst does not bleed through into channels of interest and singular primary and lysenin are not demonstrating fluorescence when they should not (because the secondary fluorescence tag is not introduced in these controls). Furthermore, the role of $\mathrm{H}+1+2$ and $\mathrm{H}+\mathrm{L}+2$ controls are to test for non-specific binding. If fluorescence is observed in the $\mathrm{H}+1+2$ case, this means primary antibody is binding to non-specific regions of the cell even though lysenin protein is not present. Similarly, if the $\mathrm{H}+\mathrm{L}+2$ controls yield fluorescence, the 
secondary antibody is non-specifically binding to the lysenin protein and other regions of the cell even when the primary antibody against lysenin is not present. Figure 39 includes unsatisfactory control images for $\mathrm{H}+1+2$ and $\mathrm{H}+\mathrm{L}+2$.

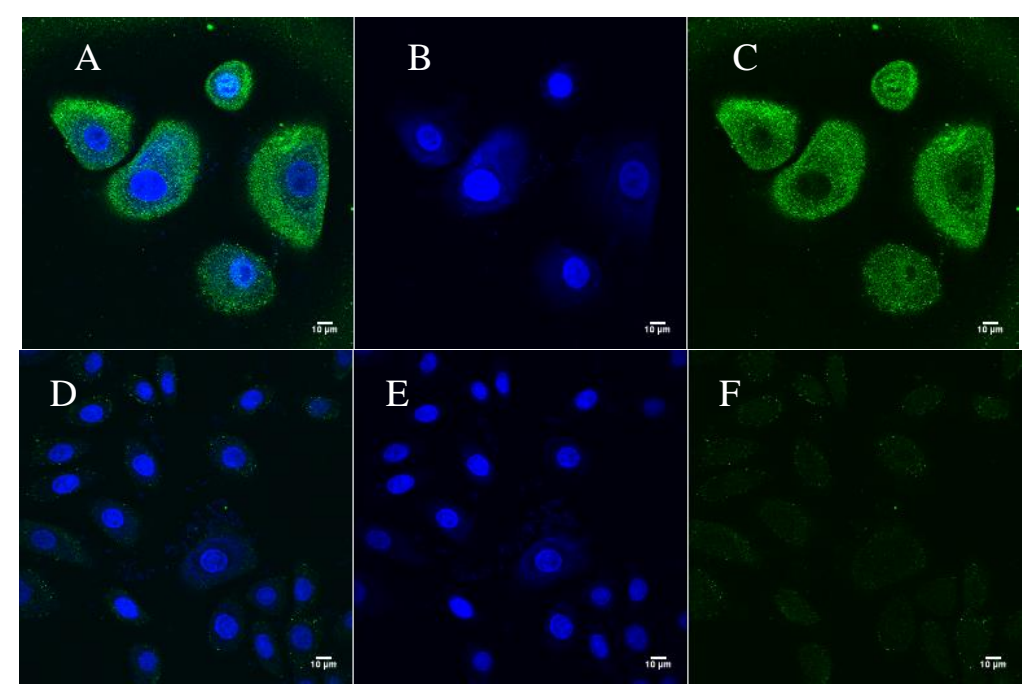

Figure 39: Control Images. (A-C) $\mathrm{H}+1+2$ demonstrating non-specific primary binding $(60 \mathrm{x}$ objective). (A) Merge, (B) DAPI, (C) AF 488. (D-F) H+L+2 demonstrating slight non-specific secondary fluorescence (60x objective). (D) Merge, (E) DAPI, (F) AF 488.

An important takeaway from the images was that bright non-specific primary binding was observed in the $\mathrm{H}+1+2$ case, meaning fluorescence was still observed even when lysenin, the protein that binds to sphingomyelin directly, was not present. This means the primary was binding randomly to locations in a cell. Further optimization was needed to attenuate the nonspecific primary binding in the $\mathrm{H}+1+2$ case.

Figure 40 includes images of the experimental images with all components of the staining procedure $(\mathrm{H}+\mathrm{L}+1+2)$. Lysenin and primary concentration were varied and overall image quality was assessed. 


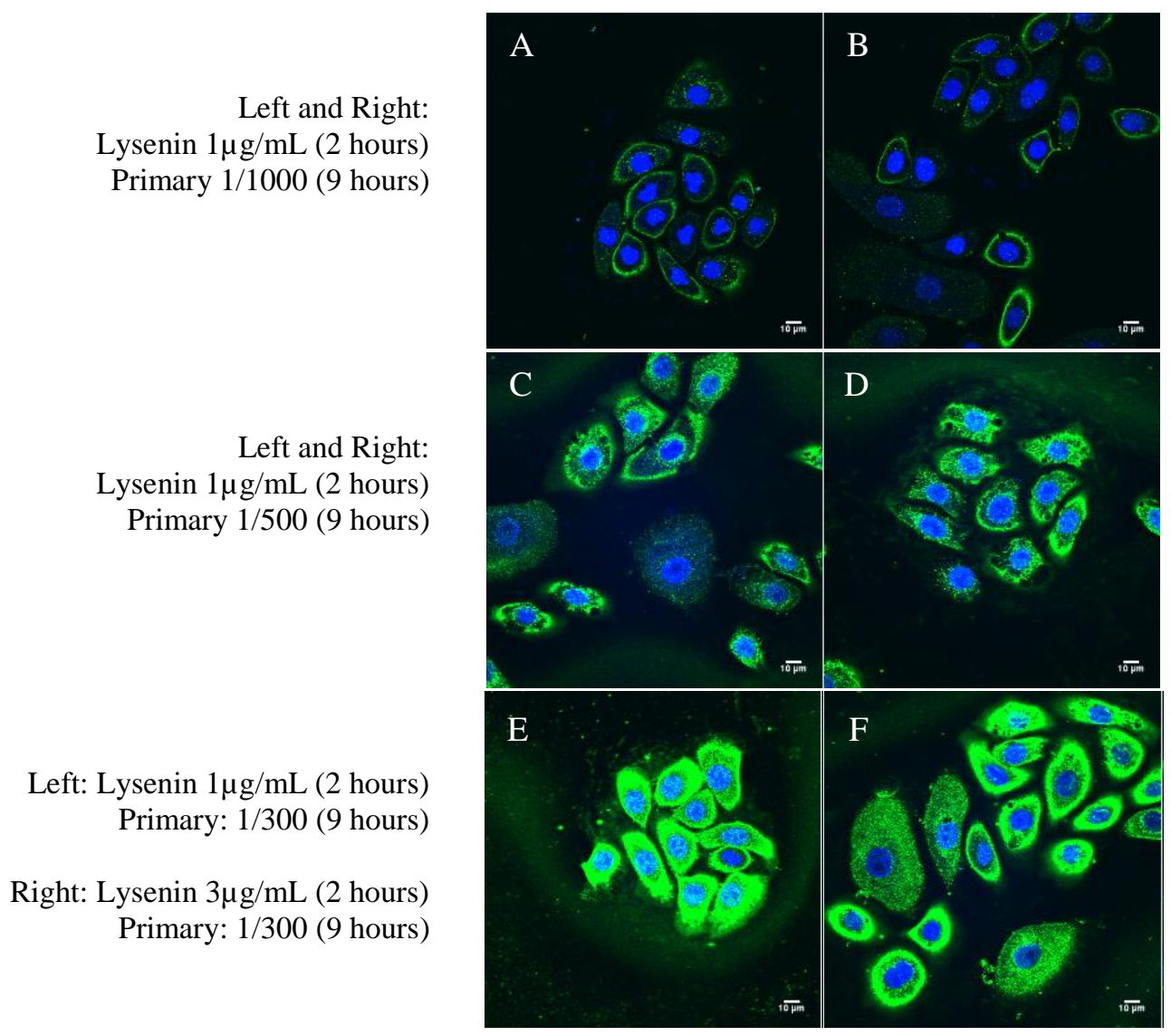

Figure 40: Experimental H+L+1+2 Images. (A-B) Two representative merge images of lysenin $(1 \mu \mathrm{g} / \mathrm{mL})$ and primary (1/1000). (C-D) Two representative merge images of lysenin $(1 \mu \mathrm{g} / \mathrm{mL})$ and primary (1/500). (E) Representative merge image of lysenin $(1 \mu \mathrm{g} / \mathrm{mL})$ and primary $(1 / 300)$.

(F) Representative merge image of lysenin $(3 \mu \mathrm{g} / \mathrm{mL})$ and primary $(1 / 300)$. 60x objective.

The figure demonstrates that bright staining and slight image oversaturation was observed for cells stained with $1 / 300$ diluted primary and/or $3 \mu \mathrm{g} / \mathrm{mL}$ lysenin protein (Figures $40 \mathrm{E}$ and $40 \mathrm{G}) .1 \mu \mathrm{g} / \mathrm{mL}$ lysenin with $1 / 1000$ primary yieded distinct plasma membrane staining (Figures 40A-B). Finally, the $1 \mu \mathrm{g} / \mathrm{mL}$ lysenin with $1 / 500$ resulted in image brightness between the two extremes.

After this initial experiment, it was decided to progress with a $1 \mathrm{ug} / \mathrm{mL}$ concentration of Lysenin and 1/500 primary dilution for 9 hours because the images matched observed staining morphology in published literature (bright plasma membrane staining) [65]. However, a subsequent experiment with the previously chosen settings still demonstrated significant nonspecific binding in the controls. Therefore, the primary incubation time was reduced to 1 hour. 
The objective for reducing the primary incubation times was to decrease the saturation of primary and decrease the likelihood of non-specific binding. A more concentrated blocking buffer, $4 \%$ BSA in PBS/4\% Goat Serum, was also introduced to the cells in a second optimization experiment with variables highlighted in Table IX. The role of the blocking buffer was to more thoroughly block free non-specific antigen sites. The goal of the following second optimization was to compare $1 / 500$ and $1 / 1000$ primary dilutions in order to choose the concentration with the least non-specific binding in the $\mathrm{H}+1+2$ control.

Table IX: Optimization 2: Comparison of Primary Concentrations

\begin{tabular}{|c|c|c|c|c|c|}
\hline \multicolumn{2}{|c|}{ IF Stain } & \multicolumn{3}{|c|}{ Concentration } & Incubation Time \\
\hline \multicolumn{2}{|c|}{ Blocking $(\mathrm{B})$} & \multicolumn{3}{|c|}{$4 \%$ BSA in PBS/4\% Goat Serum } & Overnight \\
\hline \multicolumn{2}{|c|}{ Lysenin (L) } & \multicolumn{3}{|c|}{$1 \mathrm{ug} / \mathrm{mL}$} & $2 \mathrm{hr}$ \\
\hline \multicolumn{2}{|c|}{ Anti-Lysenin Primary Ab (1) } & \multicolumn{3}{|c|}{$\begin{array}{c}1 / 500 \\
1 / 1000\end{array}$} & $\begin{array}{l}1 \mathrm{hr} \\
9 \mathrm{hr}\end{array}$ \\
\hline \multicolumn{2}{|c|}{ Secondary (2) } & \multicolumn{3}{|c|}{$1 / 300$} & $1 \mathrm{hr}$ \\
\hline \multicolumn{2}{|c|}{ Hoechst $(\mathrm{H})$} & \multicolumn{3}{|c|}{$0.05 \%$} & $15 \mathrm{~min}$ \\
\hline Control & Blocking & Lysenin & Primary & Secondary & Hoechst \\
\hline $\mathrm{H}+1+2$ & $\mathrm{X}$ & N/A & $\mathrm{X}(1 / 300,9 \mathrm{hr})$ & $\mathrm{X}(1 / 300,1 \mathrm{hr})$ & $X$ \\
\hline
\end{tabular}

Figure 41 includes representative images of $\mathrm{H}+1+2$ controls for both $1 / 1000$ and 1/500 dilution of primary. However, both primary concentrations still exhibited slight non-specific fluorescence. Therefore, in the final optimization experiment, additional blocking buffer recipes were tested (Table X). 


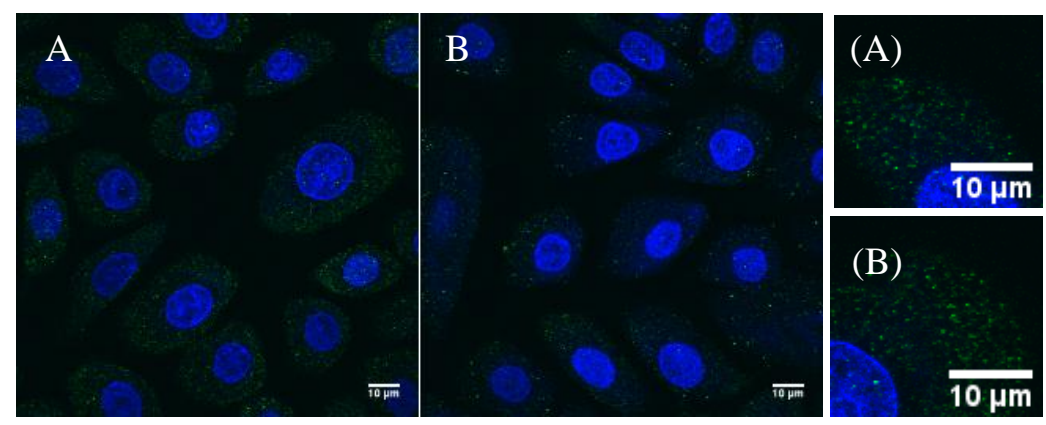

Figure 41: 1/1000 versus 1/500 Primary Dilution Comparison. (A) $\mathrm{H}+1+2$ merge with primary (1/1000). (B) H+1+2 merge with primary (1/500). Insets zoomed-in views of (A) and (B) are included in the right panel. 100x objective.

Buffers included the previously tested $4 \%$ BSA in PBS/4\% Goat Serum, but the mild detergent Tween-20, was also introduced to the blocking buffer and washing solutions to help prevent further non-specific binding by more efficiently washing off weakly adherent bonds between the primary and random binding sites. Finally, a $4 \%$ nonfat milk powder in PBS/4\% Goat Serum was introduced as an alternative blocking solution.

Table X: Optimization 3: Blocking Buffer

\begin{tabular}{|c|c|c|c|c|c|}
\hline IF Stain & \multicolumn{4}{|c|}{ Concentration } & $\begin{array}{c}\text { Incubation } \\
\text { Time }\end{array}$ \\
\hline Blocking (B) & \multicolumn{4}{|c|}{$\begin{array}{l}4 \% \text { BSA in PBS } / 4 \% \text { Goat Serum } \\
4 \% \text { BSA in PBS } / 4 \% \text { Goat Serum }+0.05 \% \text { PBS-Tween } \\
4 \% \text { Milk in PBS } / 4 \% \text { Goat Serum }+0.05 \% \text { PBS-Tween }\end{array}$} & Overnight \\
\hline Lysenin (L) & \multicolumn{4}{|c|}{$1 \mathrm{ug} / \mathrm{mL}$} & $2 \mathrm{hr}$ \\
\hline $\begin{array}{c}\text { Anti-Lysenin } \\
\text { Primary Ab (1) }\end{array}$ & \multicolumn{4}{|c|}{$1 / 1000$ in Blocking } & $1 \mathrm{hr}$ \\
\hline Secondary (2) & \multicolumn{4}{|c|}{$1 / 300$ in PBS } & $1 \mathrm{hr}$ \\
\hline Hoechst (H) & \multicolumn{4}{|c|}{$0.05 \%$ in $\mathrm{PBS}$} & $15 \mathrm{~min}$ \\
\hline Control & Blocking & Lysenin & Primary & Secondary & Hoechst \\
\hline $\mathrm{H}+1+2$ & $\mathrm{X}$ & & $\begin{array}{c}X(1 / 300, \\
9 \mathrm{hr})\end{array}$ & $\mathrm{X}(1 / 300,1 \mathrm{hr})$ & $\mathrm{X}$ \\
\hline $\mathrm{H}+\mathrm{L}+2$ & $\mathrm{X}$ & $\begin{array}{c}\mathrm{X}(1 \mathrm{ug} / \mathrm{mL}, \\
2 \mathrm{hr})\end{array}$ & & $\mathrm{X}(1 / 300,1 \mathrm{hr})$ & $\mathrm{X}$ \\
\hline
\end{tabular}




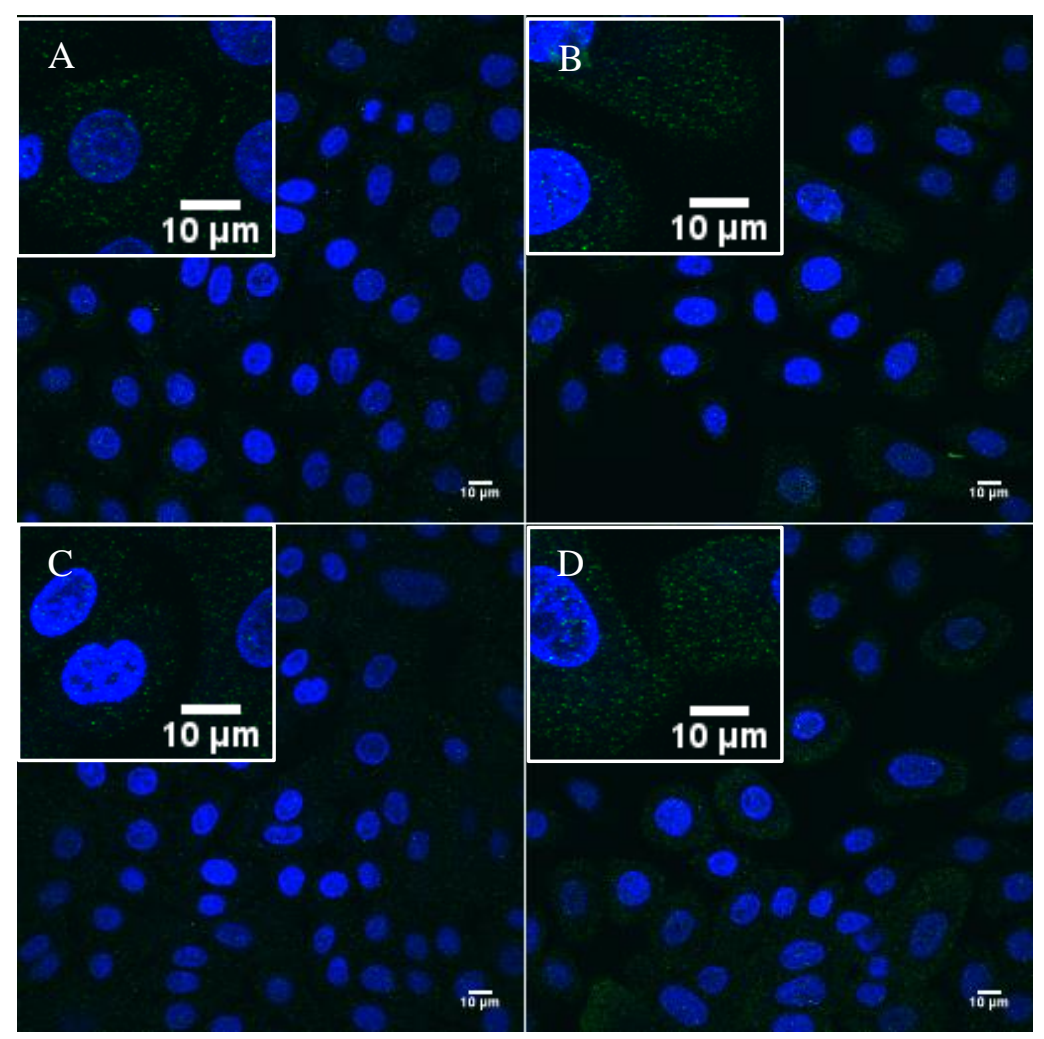

Figure 42: Blocking Buffer Optimization Non-Specific Binding for $\mathbf{H + 1 + 2}$ Control. (A) 4\%BSA/Goat, (B) 4\%BSA/Goat+PBS-Tween, (C) 4\%Milk/Goat+PBS-Tween, (D) No Blocking. 100x objective.

Figure 42 includes representative control image results of the final blocking buffer optimization. Qualitatively, all three blocking buffers reduced the fluorescence observed in the blocking conditions and yielded relatively low green fluorescence signal. Ultimately, 4\%BSA/Goat+PBS-Tween was chosen for the final, optimized blocking buffer because the BSA and goat serum are more characterized than milk. Although some slight fluorescence was observed in all images (most apparent in the Figure 42A-D insets), the signal was deemed negligible and blocking was determined to be sufficient. Lab resources and time were directed towards further characterizing the stain in dual experiments.

Finally, Figure 43 includes representative images of experimental $\mathrm{H}+\mathrm{L}+1+2$ treatments. The use of blocking and reducing primary incubations did not significantly affect staining efficiency. 
Primary Optimization

Left: Primary 1/1000 (1 hour)

Right: Primary 1/1000 (9 hours)

Blocking Optimization

Left: Blocking 4\% BSA/Goat in PBS

Right: Blocking 4\% BSA/4\% Goat in PBS-Tween

Lysenin $1 \mu \mathrm{g} / \mathrm{mL}$ ( 2 hours)

Primary 1/1000 (9 hours)

Blocking Optimization

Left: Blocking 4\% Milk/4\% Goat in PBS-Tween

Right: No blocking

Lysenin $1 \mu \mathrm{g} / \mathrm{mL}$ ( 2 hours)

Primary 1/1000 (9 hours)
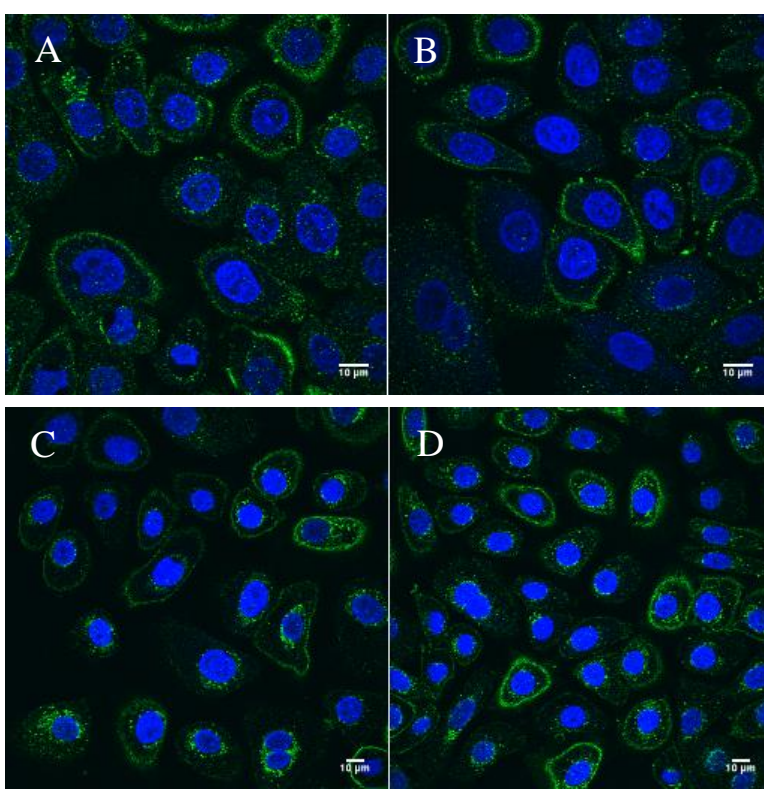

D

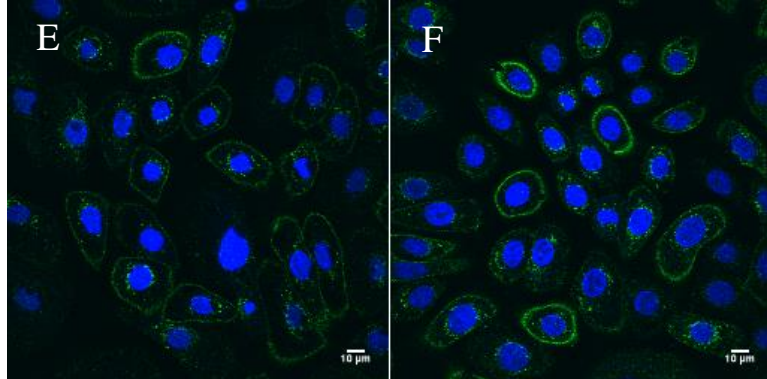

$=$

Figure 43: H+L+1+2 Staining Efficiency Comparisons During Optimization Steps. (A-B) 1/1000 Primary optimization comparison demonstrating incubating for 1 hour. (A) results in a similar staining efficiency as the 9 hour incubation (B). (C-F) Blocking optimization images (60x objective) demonstrating that all blocking varieties yielded similar images. 100x objective.

Staining efficiency did not seem to change when the primary incubation time was reduced from 9 hours to 1 hour (Figure 43A, B). Furthermore, keeping the lysenin and primary concentrations consistent, and changing the blocking variety did not yield remarkable differences in image quality. The characteristic bright plasma membrane with some intracellular expression was observed in all images. However, some heterogeneous lysenin staining among cells within the same images exists. This inconsistency has been cited in literature and is hypothesized to be a result of replicative cell senescence; KRTs from a higher passage number (P10) exhibit lower levels of lysenin staining compared to lower passage number (P4) cells [65]. Although cells with 
a relatively low passage number were used $(<\mathrm{P} 5)$ in all experiments, cell-to-cell variation could contribute to the observed heterogeneity.

\subsubsection{Discussion}

After extensive optimization experiments, the final lysenin staining parameters were optimized as the following in order to yield the best quality image (Table XI). These immunofluorescence staining parameters were utilized for subsequent dual staining with FSM.

Table XI: Final Lysenin Staining Parameters

\begin{tabular}{|c|c|c|}
\hline IF Stain & Concentration & Incubation Time \\
\hline Blocking (B) & $\begin{array}{c}4 \% \text { BSA in PBS/4\% Goat Serum + 0.05\% } \\
\text { PBS-Tween }\end{array}$ & Overnight \\
\hline Lysenin (L) & $1 \mathrm{ug} / \mathrm{mL}$ & $2 \mathrm{hr}$ \\
\hline Anti-Lysenin Primary Ab (1) & $1 / 1000$ in Blocking Solution & $1 \mathrm{hr}$ \\
\hline Secondary (2) & $1 / 300$ in PBS & $1 \mathrm{hr}$ \\
\hline Hoechst (H) & $0.05 \%$ in PBS & 15 minutes \\
\hline
\end{tabular}

The lysenin stain appears to mainly concentrate in the plasma membrane with some intracellular pockets of fluorescence. Although some very slight non-specific secondary binding was observed, the blocking capabilities of $4 \% \mathrm{BSA} / \mathrm{Goat}+\mathrm{PBS}-\mathrm{Tween}$ was deemed sufficient. Some heterogeneity was also observed among cells within the same image. These observations were consistent among all staining optimization stages and are cited in published literature to be a result of cellular senescence [65]. To confirm that cell senescence is leading to differences in lysenin staining, a cell division marker or proliferation assessment like alamarBlue ${ }^{\circledR}$ could be correlated with lysenin staining. 


\subsection{Ceramide Stain Optimization}

\subsubsection{Summary and Objectives}

The goals of these ceramide optimization studies were to identify the optimal ceramide primary antibody and secondary antibody concentration in order to reduce non-specific binding while obtaining a high signal to noise ratio. Three experiments were conducted to vary the blocking, primary concentration, and stain incubation times. Similar to the lysenin experiments, secondary concentration remained at a consistent concentration and incubation time throughout all experiments. The following results section includes a chart of experimental factors corresponding to each optimization step followed by images.

\subsubsection{Results}

Table XII includes the experimental variables tested in the first optimization study. The primary antibody concentrations were varied while incubation time and blocking remained consistent. First, fixed 8-well plates were blocked with 2\% BSA in PBS overnight, then anticeramide primary antibody was subsequently introduced at dilutions of $1 / 200$ or $1 / 300$ in PBS for

1 hour or 9 hours. Finally, a secondary antibody was added at a 1/300 concentration in PBS for 1 hour and a Hoechst counterstain was performed. Thorough washing steps with PBS were added between each staining step. Table XII also indicates the controls performed in the study, to assess whether non-specific binding or bleed through is a common occurrence. In the table, " $\mathrm{X}$ " denotes the indicated procedure was performed and "N/A" means that particular step was skipped. 
Table XII: Optimization 1: Primary and Controls

\begin{tabular}{|c|c|c|c|c|c|c|}
\hline \multicolumn{3}{|c|}{ IF Stain } & \multicolumn{2}{|c|}{ Concentration } & \multicolumn{2}{|c|}{ Incubation Time } \\
\hline \multicolumn{3}{|c|}{ Blocking $(\mathrm{B})$} & \multicolumn{2}{|c|}{$2 \%$ BSA in PBS $/ 2 \%$ Goat } & \multicolumn{2}{|c|}{ Overnight } \\
\hline \multicolumn{3}{|c|}{ Anti-Ceramide Primary Ab (1) } & \multicolumn{2}{|c|}{$1 / 200,1 / 300$} & \multicolumn{2}{|c|}{$1 \mathrm{hr}, 9 \mathrm{hr}$} \\
\hline \multicolumn{3}{|c|}{ Secondary (2) } & \multicolumn{2}{|c|}{$1 / 300$} & \multicolumn{2}{|c|}{$1 \mathrm{hr}$} \\
\hline \multicolumn{3}{|c|}{ Hoechst $(\mathrm{H})$} & \multicolumn{2}{|r|}{$0.05 \%$} & \multicolumn{2}{|c|}{$15 \mathrm{~min}$} \\
\hline Control & Blocking & & Lysenin & Primary & Secondary & Hoechst \\
\hline $\mathrm{H}$ & $\mathrm{X}$ & & N/A & N/A & N/A & $\mathrm{X}$ \\
\hline $\mathrm{H}+1$ & $\mathrm{X}$ & & N/A & $\mathrm{X}(1 / 300,9 \mathrm{hr})$ & $\mathrm{X}(1 / 300,1 \mathrm{hr})$ & $\mathrm{X}$ \\
\hline $\mathrm{H}+2$ & $\mathrm{X}$ & $\mathrm{X}(1$ & $\mathrm{ug} / \mathrm{mL}, 2 \mathrm{hr})$ & N/A & $\mathrm{X}(1 / 300,1 \mathrm{hr})$ & $\mathrm{X}$ \\
\hline
\end{tabular}

Figure 44 includes representative images of the controls $\mathrm{H}+2$ (Figure 44A) and $\mathrm{H}+1$

(Figure 44B). The ceramide was conjugated with an AF 647 secondary and appears as white in all subsequent images. Some non-specific secondary binding was observed in the $\mathrm{H}+2$ control, meaning secondary antibody was randomly binding to locations in a cell instead of selectively binding to just the ceramide primary antibody. No AF 647 ceramide channel fluorescence was observed in the $\mathrm{H}+1$ control.

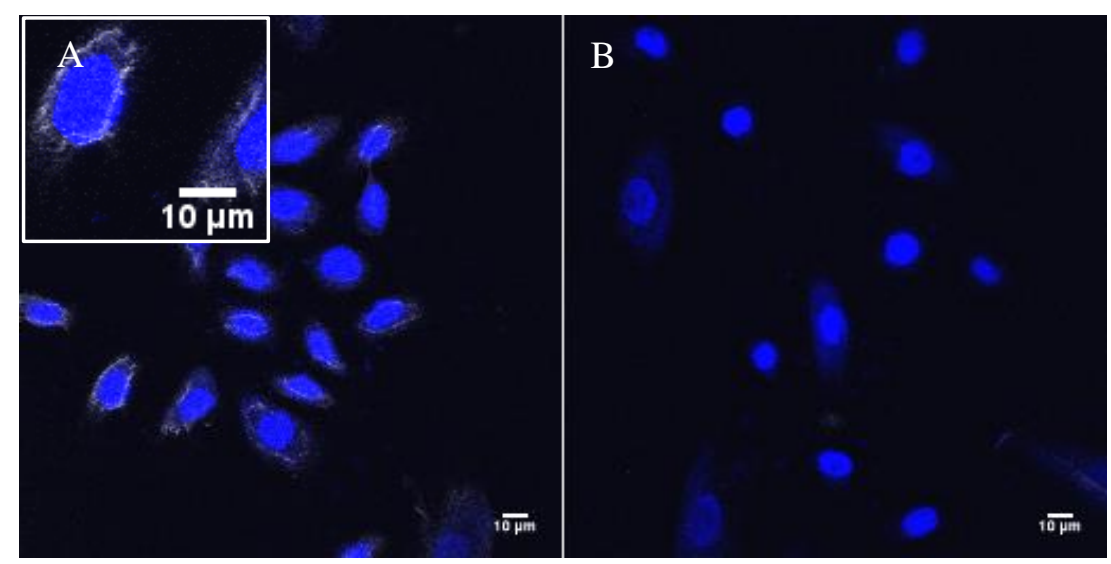

Figure 44: Control Merged Images with DAPI. (A) H+2 control exhibits non-specific secondary binding, (B) $\mathrm{H}+1$ control shows no random, unattributable fluorescence of the primary or Hoechst into the AF 647 ceramide channel. 100x objective.

Figure 45 includes representative images of $\mathrm{H}+1+2$ experimental images for the four different treatments (1/200 for 1 hour, $1 / 200$ for 9 hours, $1 / 300$ for 1 hour, 1/300 for 9 hours). Within each well, heterogeneous staining was observed. Some cells exhibited diffuse, even 
cytoplasmic staining (e.g. Figure 45C and 45D, while others had bright, uneven pockets or ceramide expression (e.g. Figure 45B).

Primary: 1/200 (1 hour)

Primary: 1/200 (9 hours)

Primary: 1/300 (1 hour)
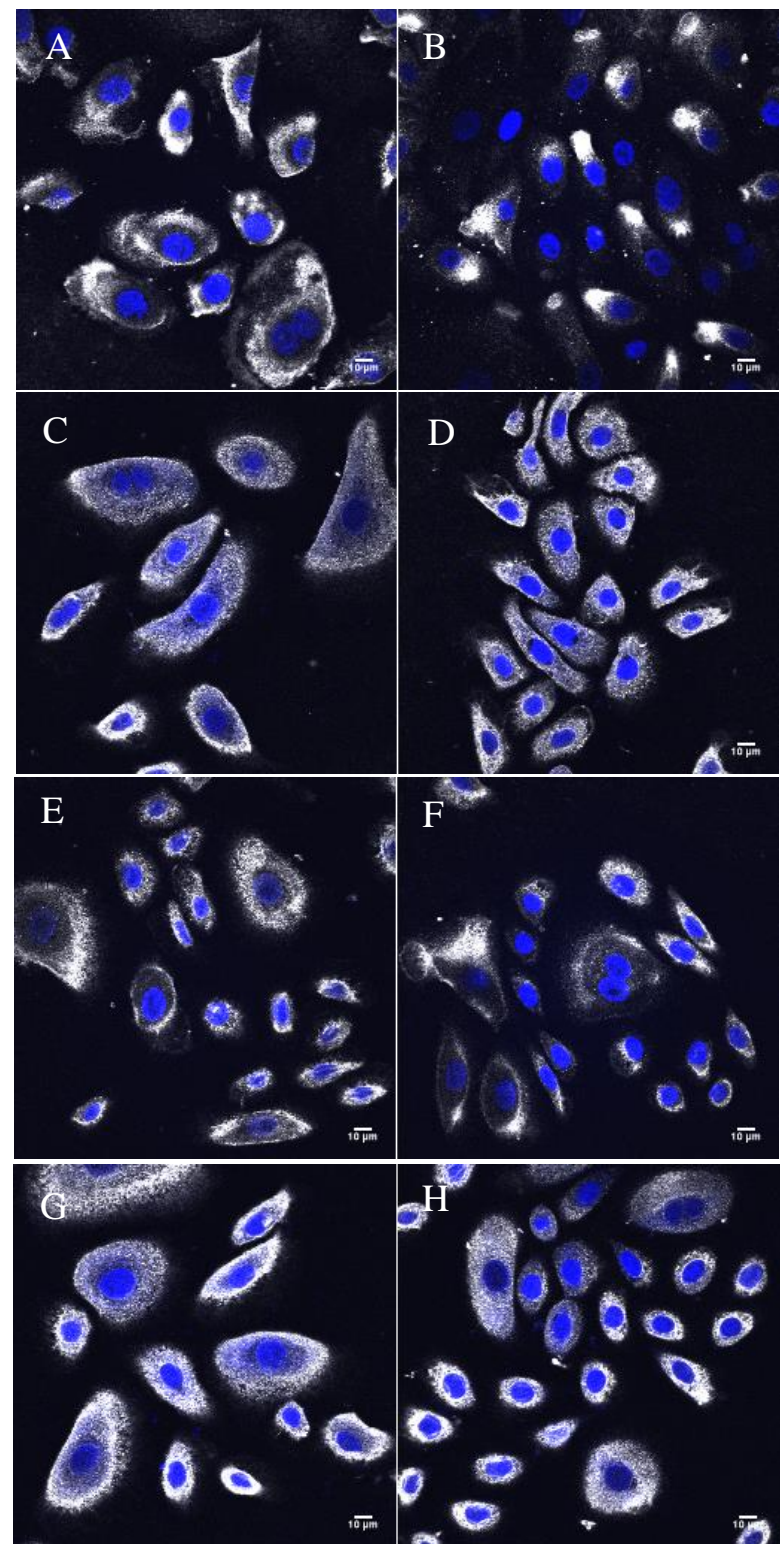

Figure 45: Experimental H+1+2 Images. (A-B) Two representative merge images of primary (1/200) for 1 hour. (C-D) Two representative merge images of primary (1/200) and 9 hours. (E-F) Two representative merge image of primary (1/300) for 1 hour. (G-H) Two representative merge image of primary (1/300) for 9 hours. Within each treatment, inconsistent ceramide results were observed. Some cells had diffuse ceramide staining, while others exhibited pockets of intense, bright staining. 100x objective. 
Moving forward, the 1/300 primary concentration for 9 hours was selected because no differences in signal brightness was observed between the concentration variables, and 1/300 conserves more reagent. Furthermore, 9 hours was chosen as the incubation timepoint because the image results suggested the cells were stained more evenly and consistently than the 1 hour staining treatment (Figure 45C versus $45 \mathrm{~A}$ and Figure $45 \mathrm{G}$ versus $45 \mathrm{E}$ ). Incubating for a longer time period allows more stain to penetrate the cell and potentially reduces heterogeneous staining occurrences. In the second follow-up optimization study, more blocking buffer variants (4\% Goat in PBS, 4\% BSA in PBS, and 4\% BSA/4\% Goat) were tested to reduce the non-specific secondary binding observed in the $\mathrm{H}+2$ control (Table XIII).

Table XIII: Blocking to Reduce Non-Specific Secondary Binding

\begin{tabular}{|c|c|c|c|c|c|c|}
\hline \multicolumn{3}{|c|}{ IF Stain } & \multicolumn{2}{|c|}{ Concentration } & \multicolumn{2}{|c|}{ Incubation Time } \\
\hline \multicolumn{3}{|c|}{ Blocking (B) } & \multicolumn{2}{|c|}{$\begin{array}{l}\text { 4\% Goat in PBS } \\
4 \% \text { BSA in PBS } \\
4 \% \text { BSA } / 4 \% \text { Goat }\end{array}$} & \multicolumn{2}{|c|}{ Overnight } \\
\hline \multicolumn{3}{|c|}{ Anti-Ceramide Primary Ab (1) } & \multicolumn{2}{|c|}{$1 / 300$} & \multicolumn{2}{|c|}{$9 \mathrm{hr}$} \\
\hline \multirow{2}{*}{\multicolumn{3}{|c|}{$\begin{array}{c}\text { Secondary (2) } \\
\text { Hoechst }(\mathrm{H})\end{array}$}} & \multicolumn{2}{|c|}{$1 / 300$} & \multicolumn{2}{|c|}{$1 \mathrm{hr}$} \\
\hline & & & \multicolumn{2}{|c|}{$0.05 \%$} & \multicolumn{2}{|c|}{$15 \mathrm{~min}$} \\
\hline \multicolumn{3}{|c|}{\begin{tabular}{l|l} 
Control & Blocking
\end{tabular}} & Lysenin & Primary & Secondary & Hoechst \\
\hline $\mathrm{H}$ & $\mathrm{X}$ & & & & & $\mathrm{X}$ \\
\hline $\mathrm{H}+1$ & $\mathrm{X}$ & & & $\mathrm{X}(1 / 300,9 \mathrm{hr})$ & $\mathrm{X}(1 / 300,1 \mathrm{hr})$ & $\mathrm{X}$ \\
\hline $\mathrm{H}+2$ & $\mathrm{X}$ & $\mathrm{X}($ & lug/mL, 2hr) & & $\mathrm{X}(1 / 300,1 \mathrm{hr})$ & $\mathrm{X}$ \\
\hline
\end{tabular}

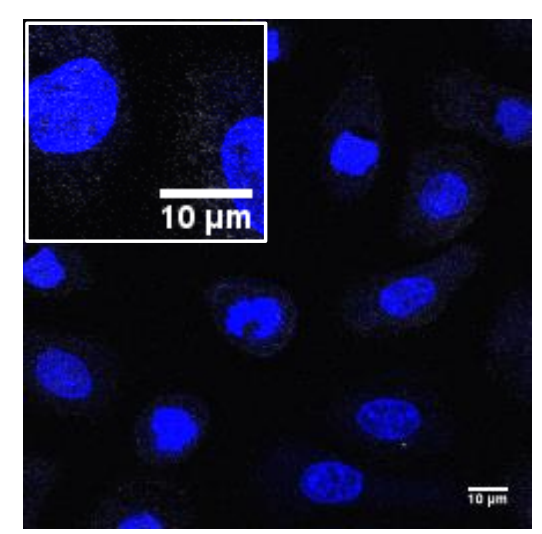

Figure 46: Control H+2 with 4\% BSA/4\% Goat Serum Blocking Merge Image with DAPI. No non-specific secondary staining is observed. 100x objective. 
Figure 46 depicts an image of a successful control with the 4\% BSA/4\% Goat Serum Blocking solution. In the last optimization experiment, primary ceramide antibody $1 / 300$ and 1/400 dilution concentrations were compared to further optimize the primary concentration and potentially save more reagent. Table XIV lists all other experimental factors and controls. Only primary concentration was modified.

Table XIV: Further Reduction in Primary Concentration

\begin{tabular}{|c|c|c|c|c|c|c|}
\hline \multicolumn{3}{|c|}{ IF Stain } & \multicolumn{2}{|c|}{ Concentration } & \multicolumn{2}{|c|}{ Incubation Time } \\
\hline \multicolumn{3}{|c|}{ Blocking (B) } & \multicolumn{2}{|c|}{$4 \%$ BSA $/ 4 \%$ Goat } & \multicolumn{2}{|c|}{ Overnight } \\
\hline \multicolumn{3}{|c|}{ Anti-Ceramide Primary Ab (1) } & \multicolumn{2}{|c|}{$\begin{array}{l}1 / 300 \\
1 / 400\end{array}$} & \multicolumn{2}{|c|}{$9 \mathrm{hr}$} \\
\hline \multicolumn{3}{|c|}{ Secondary (2) } & \multicolumn{2}{|c|}{$1 / 300$} & \multicolumn{2}{|c|}{$1 \mathrm{hr}$} \\
\hline \multicolumn{3}{|c|}{ Hoechst $(\mathrm{H})$} & \multicolumn{2}{|c|}{$0.05 \%$} & \multicolumn{2}{|c|}{$15 \mathrm{~min}$} \\
\hline Control & Blocking & & Lysenin & Primary & Secondary & Hoechst \\
\hline $\mathrm{H}$ & $\mathrm{X}$ & & N/A & N/A & N/A & $\mathrm{X}$ \\
\hline $\mathrm{H}+1$ & $\mathrm{X}$ & & N/A & $\mathrm{X}(1 / 300,9 \mathrm{hr})$ & $\mathrm{X}(1 / 300,1 \mathrm{hr})$ & $\mathrm{X}$ \\
\hline $\mathrm{H}+2$ & $\mathrm{X}$ & $\mathrm{X}($ & $1 \mathrm{ug} / \mathrm{mL}, 2 \mathrm{hr})$ & N/A & $\mathrm{X}(1 / 300,1 \mathrm{hr})$ & $\mathrm{X}$ \\
\hline
\end{tabular}

Figure 47 demonstrates that no difference in ceramide stain brightness or morphology was observed between the $1 / 300$ and 1/400 primary concentrations. Both conditions yielded heterogeneous images with diffuse cytoplasmic staining in some images (Figure 47A, C) and patchy staining in others (Figure 47B, D). The 1/400 dilution was selected as the final ceramide concentration in order to conserve reagent and no difference was observed between 1/400 and the higher concentration. 


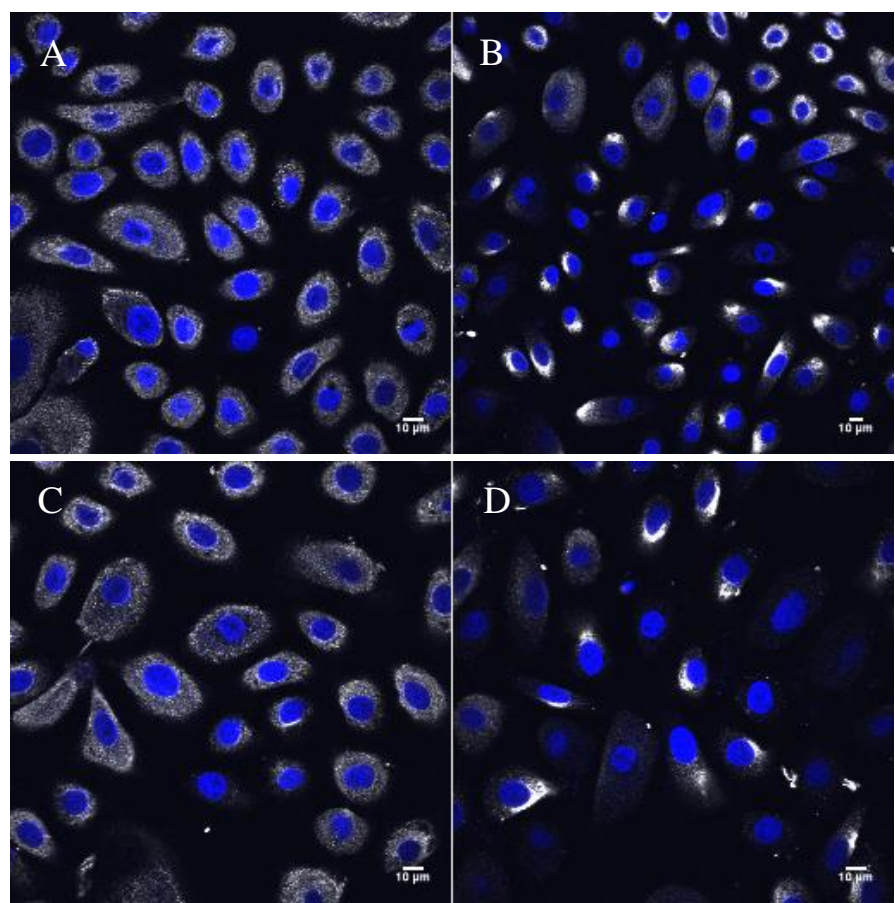

Figure 47: 1/300 versus 1/400 Ceramide Primary Comparison. (A) 1/300 dilution (60x objective) demonstrating diffuse staining. (B) 1/300 dilution (40x objective) with patchy staining. (C) 1/400 dilution (60x objective) with diffuse staining. (D) 1/400 dilution (60x objective) with patchy staining. The ceramide stain again displayed inconsistent cytoplasmic expression.

\subsubsection{Discussion}

After several optimization experiments, the final ceramide staining parameters were optimized as indicated in Table XV in order to yield the best quality image:

\section{Table XV: Optimized Ceramide Immunofluorescence Parameters}

\begin{tabular}{|c|c|c|}
\hline IF Stain & Concentration & Incubation Time \\
\hline Blocking (B) & $4 \%$ BSA in PBS/4\% Goat Serum & Overnight \\
\hline Anti-Ceramide Primary Ab (1) & $1 / 400$ in Blocking Solution & $1 \mathrm{hr}$ \\
\hline Secondary (2) & $1 / 300$ in PBS & $1 \mathrm{hr}$ \\
\hline Hoechst $(\mathrm{H})$ & $0.05 \%$ in PBS & 15 minutes \\
\hline
\end{tabular}

These parameters will be utilized for subsequent dual staining with FSM and lysenin. The ceramide stained cells also demonstrated heterogeneous staining morphologies which was observed despite varying blocking, primary concentrations, and primary incubation time. The reason for the inconsistent staining is unknown at this time, but could be a result of the replicative 
senescence hypothesis cited in the lysenin optimization studies [65]. In addition, few research groups employ immunofluorescence to stain ceramide, therefore a deficit of direct image comparisons exists. The localized pockets of inconsistently stained cells within the same well could indicate some biological or regional cell signaling effecting ceramide concentration in a cell. Senescence or proliferation markers could be correlated with ceramide to visualize any staining patterns. Further investigation into the inconsistency will be directed to future work for the lab, outside the scope of this thesis. 


\subsection{Dual Lysenin, Ceramide, and FSM Staining}

\subsubsection{Summary and Objectives}

Dual immunofluorescence staining was performed with two main experiments focused first on exploratory qualitative assessment, and second, on quantitative fluorescence distributions of FSM, lysenin, and ceramide together. The goal of this first dual stain experiment study was mainly proof-of-concept to determine whether dual immunofluorescence staining of FSM treated cells with both lysenin and ceramide was possible. Dual staining with lysenin and ceramide immunofluorescence is novel and currently no published primary literature characterizes how ceramide and endogenous SM levels fluctuate in a cell after continuous incubations with exogenous FSM using immunofluorescence and imaging. Being able to visualize intracellular distributions of endogenous SM, ceramide, and FSM in a cell could potentially elucidate the cell's response to exogenous SM treatments. Optimization of the dual staining protocol is warranted for future UV experiments to determine how lysenin, ceramide, and FSM change with UV perturbation.

Given the time and resources available, only one exploratory experiment was performed prior to the main quantitative fluorescence trafficking study, and the optimized lysenin and ceramide protocols were used. 8-well plates with monolayers of KRTs were independently incubated with FSM at $37^{\circ} \mathrm{C}$ for 24 hours. Immediately after treatment, cells were gently washed once with PBS then fixed with $3.75 \%$ PFA. Using the previously optimized immunofluorescence protocols for lysenin and ceramide, FSM treated cells were stained individually with lysenin and ceramide, then dual stained with both. Individual immunofluorescence followed previously established protocol. For the dual staining, plates were again blocked overnight at $4^{0} \mathrm{C}$ with $4 \%$ BSA/4\% Goat diluted in PBS-Tween. Next, the lysenin protein was incubated for 2 hours, washed, and followed by the lysenin primary antibody for 1 hour. Then the ceramide antibody 
was incubated for 9 hours, followed by simultaneous secondary incubations (AF 647 goat antimouse and AF 594 goat anti-rabbit) introduced to the cells at their respective concentrations. Finally, the nuclei were stained with Hoechst. Sufficient washing steps were performed between each step in the protocol. During imaging, z-stacks with a $1 \mu \mathrm{m}$ step size were obtained to generate a 3D picture of cell staining.

\subsubsection{Results}

Figure 48 shows representative images for FSM and lysenin staining. Consistent with previous experiments, lysenin mainly stained the plasma membrane, but some intracellular fluorescence clusters were observed in a few cells (Figure 48A). Again, some heterogeneity was observed with some cells brightly stained with lysenin and others not. The FSM signal was still observed after immunofluorescence and the merge image with lysenin (Figure 48C) suggesting future colocalization analysis is possible. 


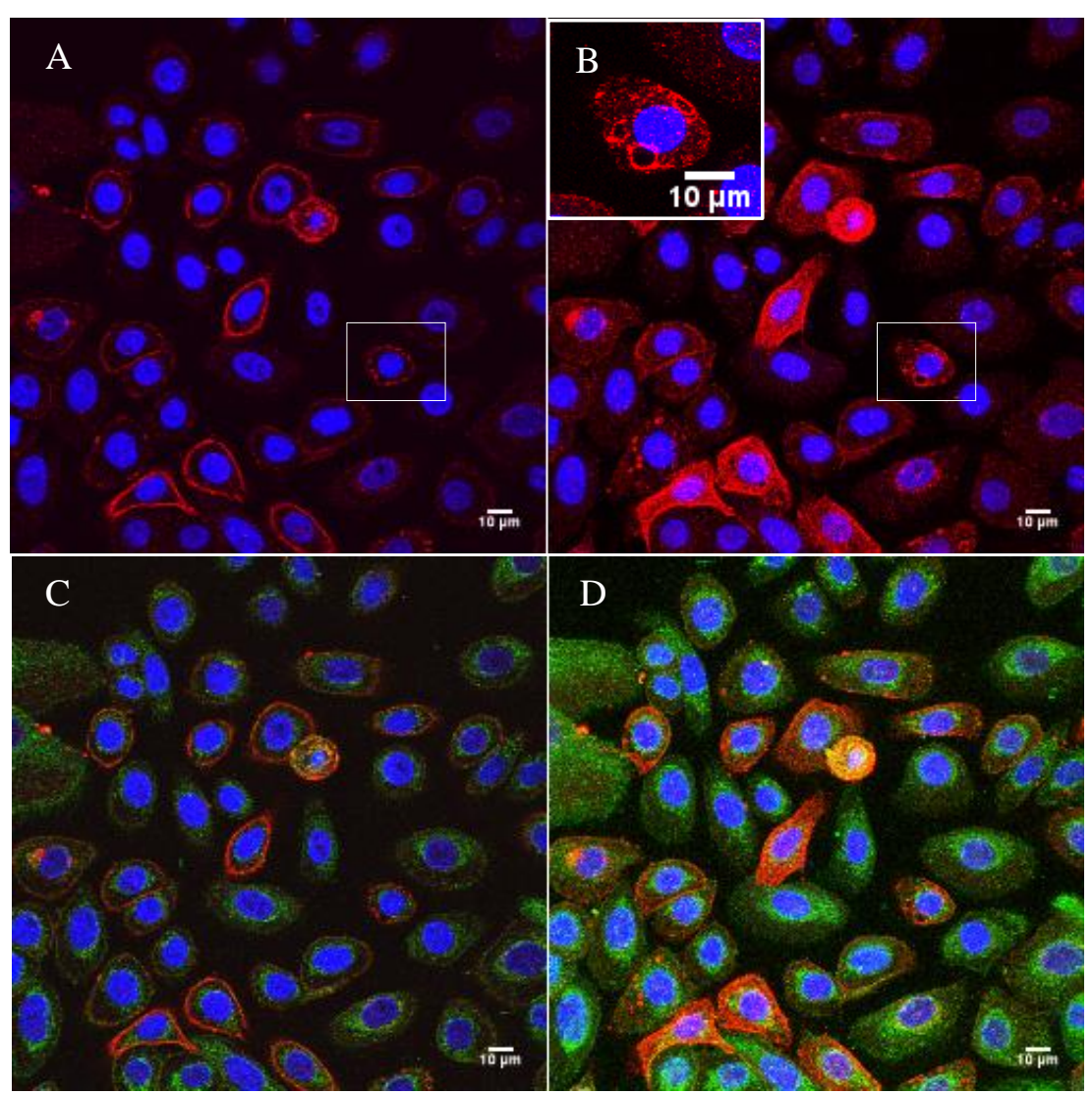

Figure 48: 2D and Maximal Z-Projections of FSM and Lysenin Immunofluorescence. (A) 2D image of AF 594 lysenin channel. Staining mostly concentrates along the plasma membrane (B) Z-projection of lysenin channel. Adding the 3D component to the images better represents lysenin distribution. (B) inset shows some regions of concentrated lysenin fluorescence (C) Merge 2D image AF 594 lysenin with AF 488 FSM. (D) Merge z-projection of lysenin and FSM. 100x objective.

Since z-stacks were obtained for each image, Figure 48B and D show maximal 3D zprojections of the z-stacks. 3D images are useful to show fluorescence distribution across the entire cell volume. Comparing 2D (Figure 48A) to the 3D projection (Figure 48B), a bright plasma membrane translates to a bright shell around the nucleus because the projection also includes plasma membrane on top of the nucleus that was excluded in the $2 \mathrm{D}$ confocal image. The boxed cell zoom inset further demonstrates the utility of these 3D renderings in showing bright pockets of concentrated lysenin fluorescence not visible with the 2D images. Furthermore, colocalization of the FSM and lysenin is easier to visualize by the bright orange and yellow shells surrounding the nucleus. 


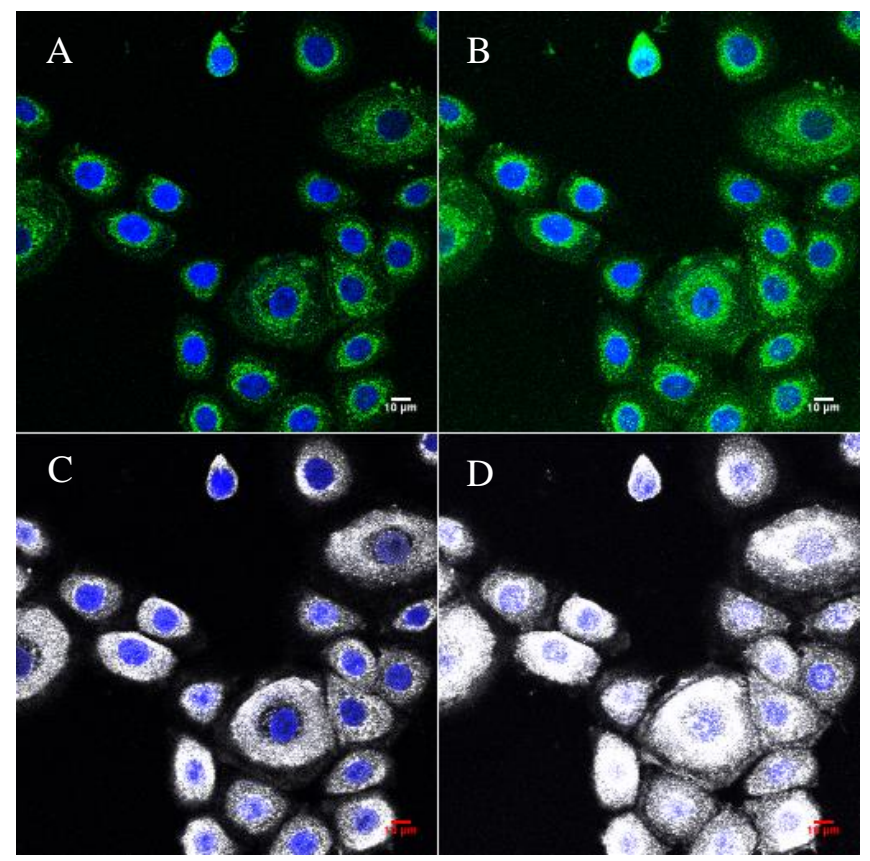

Figure 49: 2D and Maximal Z-Projections of FSM and Ceramide Immunofluorescence. (A) 2D image of AF 488 FSM channel. (B) Z-projection of AF 488 FSM channel. (C) 2D image of AF647 ceramide. (D) Z-projection of AF 647 ceramide channel. 100x objective.

Figure 49 demonstrates that ceramide immunofluorescence staining with FSM was also successful because both fluorescence signals were visualized in both the AF 488 and AF 647 channels. The z-projection images (Figure 49B and D) are again useful for visualizing holistic cell fluorescence. For example, the 3D image collects all ceramide fluorescence above the nucleus (Figure 49D). Entire cell volume images will most likely prove valuable for future fluorescence distribution and colocalization analysis.

Based on qualitative visual assessment of all images, two staining phenotypes were observed for both lysenin and ceramide (Figure 50). First, lysenin staining was either concentrated in the plasma membrane (Figure 50A-C), or intracellular in bright pockets (Figure 50D-F). 

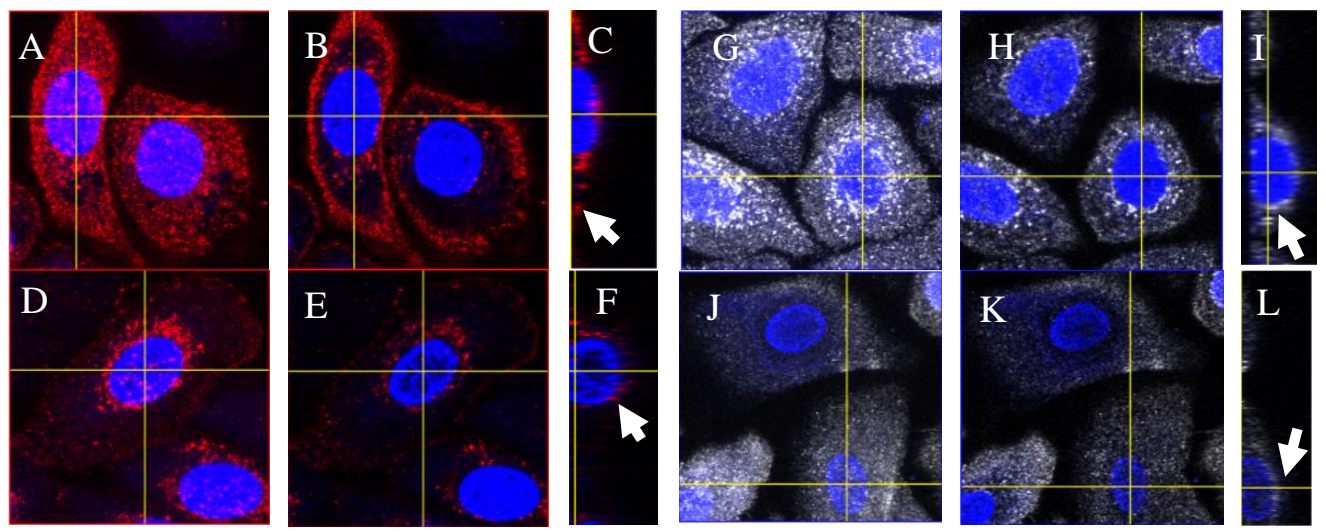

Figure 50: Differential Staining of Lysenin and Ceramide. (A-C) Bright plasma membrane lysenin staining as demonstrated in the z-projection (A), 2D image (B), and orthogonal view (C)

which shows distinct fluorescence far from the nucleus (white arrow). (D-F) Bright lysenin staining directly around the nucleus as demonstrated by the z-projection (D), 2D image (E), and orthogonal view $(\mathrm{F})$ which shows distinct fluorescence only close to the nucleus (white arrow). (G-I) Distinct plasma membrane and intracellular ceramide staining as demonstrated in the zprojection $(\mathrm{G}), 2 \mathrm{D}$ image $(\mathrm{H})$, and orthogonal view (I) which shows fluorescence clustered around the nucleus (white arrow). (J-L) Absence of a definitive ceramide fluorescence signal as demonstrated in the z-projection $(\mathrm{J}), 2 \mathrm{D}$ image $(\mathrm{K})$, and orthogonal view $(\mathrm{L})$ which only shows a slight signal above the nucleus (white arrow). 100x objective.

The orthogonal views (Figures 50C\&F for lysenin and Figures 50I\&L for ceramide) helped with visualization and confirmed the observations in both 2D slices and z-stacks. In a similar manner, ceramide also demonstrated two different staining characteristics observed within each treatment. Ceramide staining either yielded diffuse staining with some concentrated fluorescence around the nucleus (Figure 50G-I), or pockets towards the edges of the cell with varying degrees of brightness (Figure 50J-L). It is important to note that some cells within the same image field of view sometimes completely lacked fluorescence staining.

Finally, Figure 51 includes images of dual immunofluorescence staining for both lysenin, ceramide, and FSM. With the current protocol, the FSM signal appeared to disintegrate and was severely degraded (Figure 51A\&D). The laser power and sensitivity of the confocal PMT was manually increased to detect the demonstrated fluorescence levels in Figure 51B\&D. 


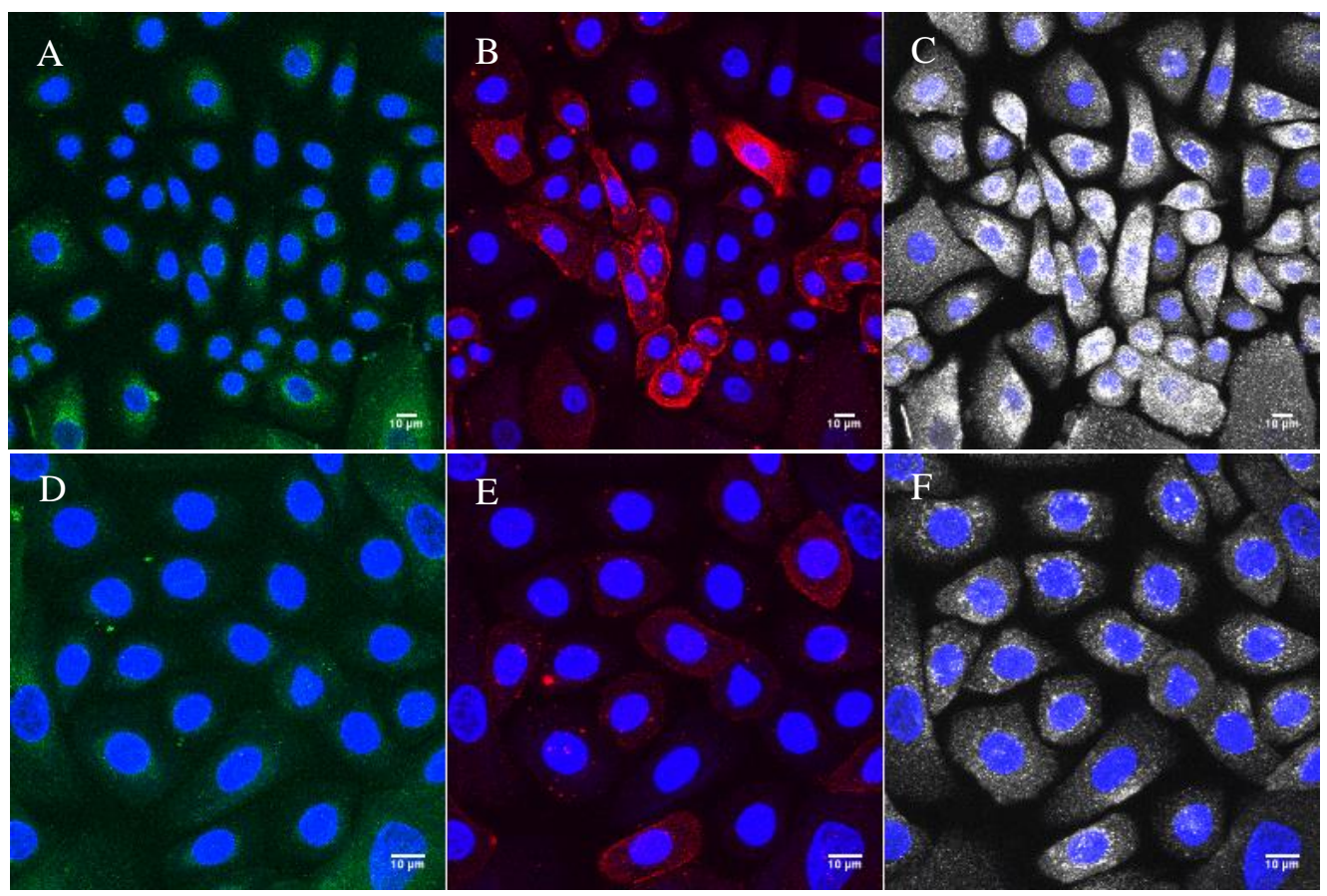

Figure 51: Z-Projection of FSM, Lysenin, and Ceramide after Dual Immunostaining. (A-C) 60x objective image. (A) FSM demonstrating significant signal degradation, (B) Lysenin, (C) Ceramide. (D-F) 100x objective. (D) FSM, (E) Lysenin with pocket of weakly stained cells, (F) Ceramide.

The lysenin and ceramide signals did not seem to change after dual staining. Lysenin was still heterogeneous, with some bright plasma membrane. Ceramide was either diffuse or concentrated in pockets. The reason for the FSM signal degradation was unknown and many factors could have contributed to the signal degradation (i.e. time, extra wash steps, using excessive detergent). The Tween-20 detergent added to the blocking and wash buffers was hypothesized to contribute to FSM degradation.

\subsubsection{Discussion}

Dual immunostaining was successful because all three signals (FSM, lysenin, ceramide) were visible in the images. However, significant signal degradation of the FSM stain was observed, warranting future troubleshooting to elucidate the reason. 


\subsection{Troubleshooting FSM Degradation Observed after Dual Immunofluorescence}

\subsubsection{Summary and Objectives}

The goal of the following experiment was to formulate a hypothesis for the FSM signal degradation observed during imaging. Multiple hypotheses can potentially explain this observation. 1) Time - FSM signal degrades over time when stored in PBS between experiments prior to imaging, with timepoints ranging from immediately after staining to up to two weeks. 2) Detergent - introducing extra Tween-20 detergent into the blocking and washing buffers leached FSM from the cell. 3) Protocol specific - the extra PBS washing steps could slowly remove the FSM signal. Furthermore, the addition of two antibodies could mask the FSM signal. The reason for decreased FSM is unknown, but the goal of the following experiment was to assess the effects of introducing Tween-20 to the blocking buffer and PBS wash. Tween-20 was identified as the target for the first optimization because its removal from the existing protocol was easier to implement. Briefly, FSM was introduced to cells at $37^{\circ} \mathrm{C}$ for 1 hour, then the media was washed once with PBS and fixed. Cells were subsequently permeabilized with Triton X and either washed with PBS or $0.05 \%$ PBS-Tween. A control without permeabilization and washing was used to assess the extent of photobleaching.

\subsubsection{Results}

As the images demonstrate in Figure 52, introducing Tween-20 washes to the image system induced some degradation, as indicated by the reduced green fluorescence brightness in Figure 52C. The degradation in FSM signal was visibly greater than the Triton X with PBS wash treatment (Figure 52B). This result suggests that Tween-20 detergent in the blocking buffer and washing steps could potentially contribute to some signal degradation observed in the dual immunofluorescence images. 


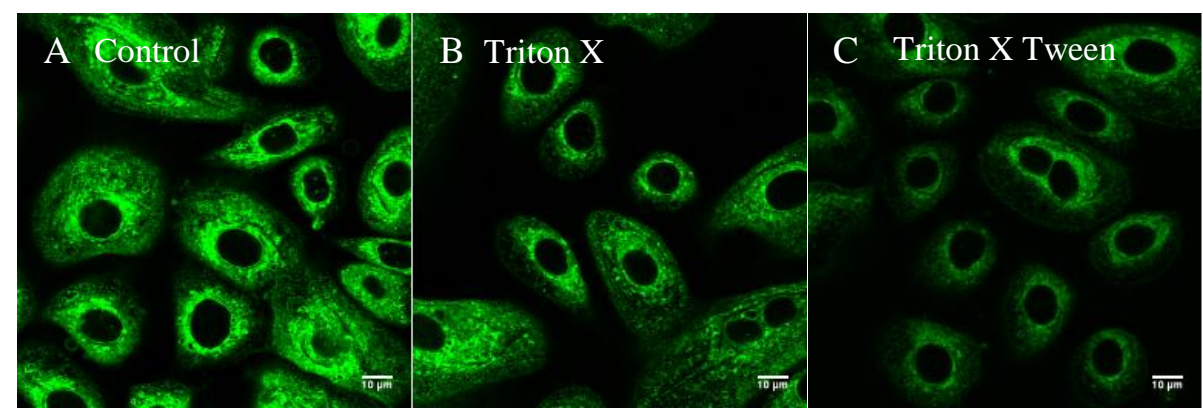

Figure 52: Signal Degradation from Various Staining Treatments. (A) Control, (B), Triton $X$ permeabilization with PBS washes, (C) Triton X permeabilization with $0.05 \%$ Tween-20 Washes. 100x objective.

\subsubsection{Discussion}

Although a decrease in signal was observed after adding a $0.05 \%$ Tween-20 to PBS wash, this troubleshooting experiment failed to recapitulate all the other variables in the dual immunostaining procedure. Tween-20 is merely one piece of the degradation dilemma, because dual stained FSM images exhibited significantly more photobleaching than what was observed in this troubleshooting experiment (Figure 53). For example, Figure 53A shows the FSM signal using the FSM laser power settings employed during 2D fluorescence trafficking experiments (Table IV). Figure 53B is an image of FSM incubated with cells for 1 hour then immediately fixed and imaged using the same laser power settings from Figure 53A and all fluorescence trafficking experiments. Finally, Figure 53C is an image of the same cells from Figure 53B, except the imaging settings were modified to match the laser power and PMT sensitivities used to obtain the images in Figure 51A\&D (dual immunofluorescence settings Table V). The images demonstrate that dual immunostaining drastically degrades the FSM signal (lack of signal in Figure 53A). This means the laser and PMT settings need to be increased in order to detect a signal (as demonstrated by severe saturation of Figure 53C when Figure 53B was imaged with the new image settings). 


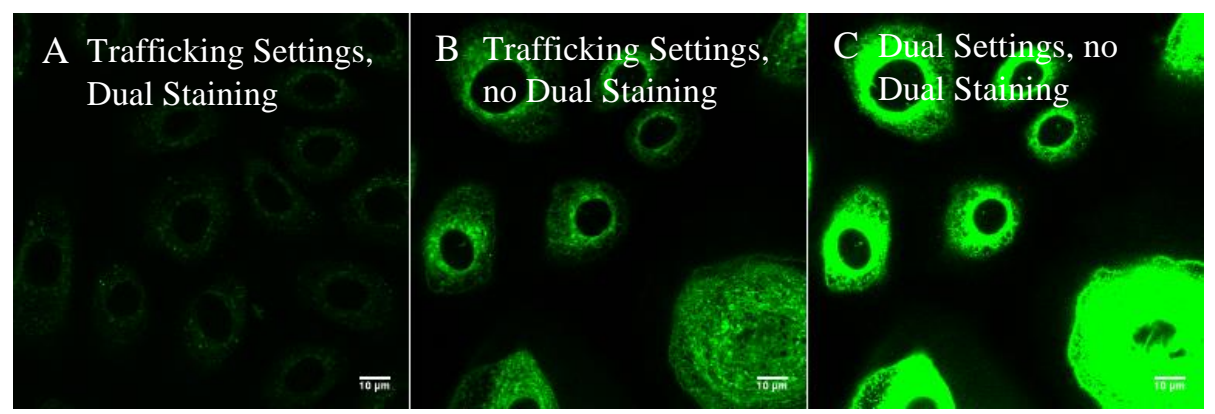

Figure 53: Additional Signal Degradation Demonstrations. (A) Dual immunofluorescence FSM channel imaged using trafficking settings. (B) Normal FSM with no extra treatment imaged using trafficking settings (no dual staining). (C) Same FSM image but with dual immunofluorescence settings. 100x objective.

Additional explanations for the diminished fluorescence could be FSM degradation in PBS during storage over time or numerous washing steps during the protocol. Further troubleshooting to identify the source of FSM degradation is currently being investigated in the lab but is outside the scope of this thesis. For the final experiment - FSM, lysenin, and ceramide fluorescence distribution - an increased laser power and PMT sensitivity was consistently used to image the FSM. 


\subsection{Dual Lysenin, Ceramide, and FSM Fluorescence Trafficking}

\subsubsection{Summary and Objectives}

The goal of final dual immunostaining protocol was to quantitively determine if FSM, lysenin (endogenous SM), and ceramide localization in a cell varied over a spectrum of continuous incubation times (10 minutes, 1 hour, 2 hour, 12 hour, 24 hour, and control without exogenous FSM incubation). The same 8-well plates used for FSM fluorescence distribution in Experiment 4.4 were dual immunostained for both lysenin and ceramide according to previously established protocol. Four wells $(n=4)$ per treatment ( 3 images/well) were imaged on the confocal and z-stacks with $1 \mu \mathrm{m}$ step sizes were obtained using identical laser settings. 2D images were post-processed in ImageJ using the trafficking and rotation ImageJ and MATLAB programs to obtain the mean gray values over normalized distances from the nucleus for all three stains. The ceramide channel was used to create the cytoplasmic ROI which was then applied to the FSM and lysenin channels. Finally, the area under each curve was calculated using MATLAB CUM TRAPZ function and plotted. ANOVA and Tukey HSD post-hoc tests were performed on the area data.

\subsubsection{Results}

Figure 54 includes representative images of FSM, ceramide, and lysenin after 10 minutes, 1 hour, 2 hour, and 12 hour continuous incubations. The 24 hour and control timepoints were not analyzed because of high variability among all three stains which made determining each cell ROI difficult. Two representative images for the 1 hour and 2 hour timepoints are included to demonstrate ceramide staining inconsistency observed throughout all timepoints. The only noticeable differences among all the channels was within the lysenin staining. A brighter plasma membrane fluorescence was observed in the 1 hour cases. Furthermore, in the 2 hour cases, more intracellular fluorescence around the nucleus was apparent. 


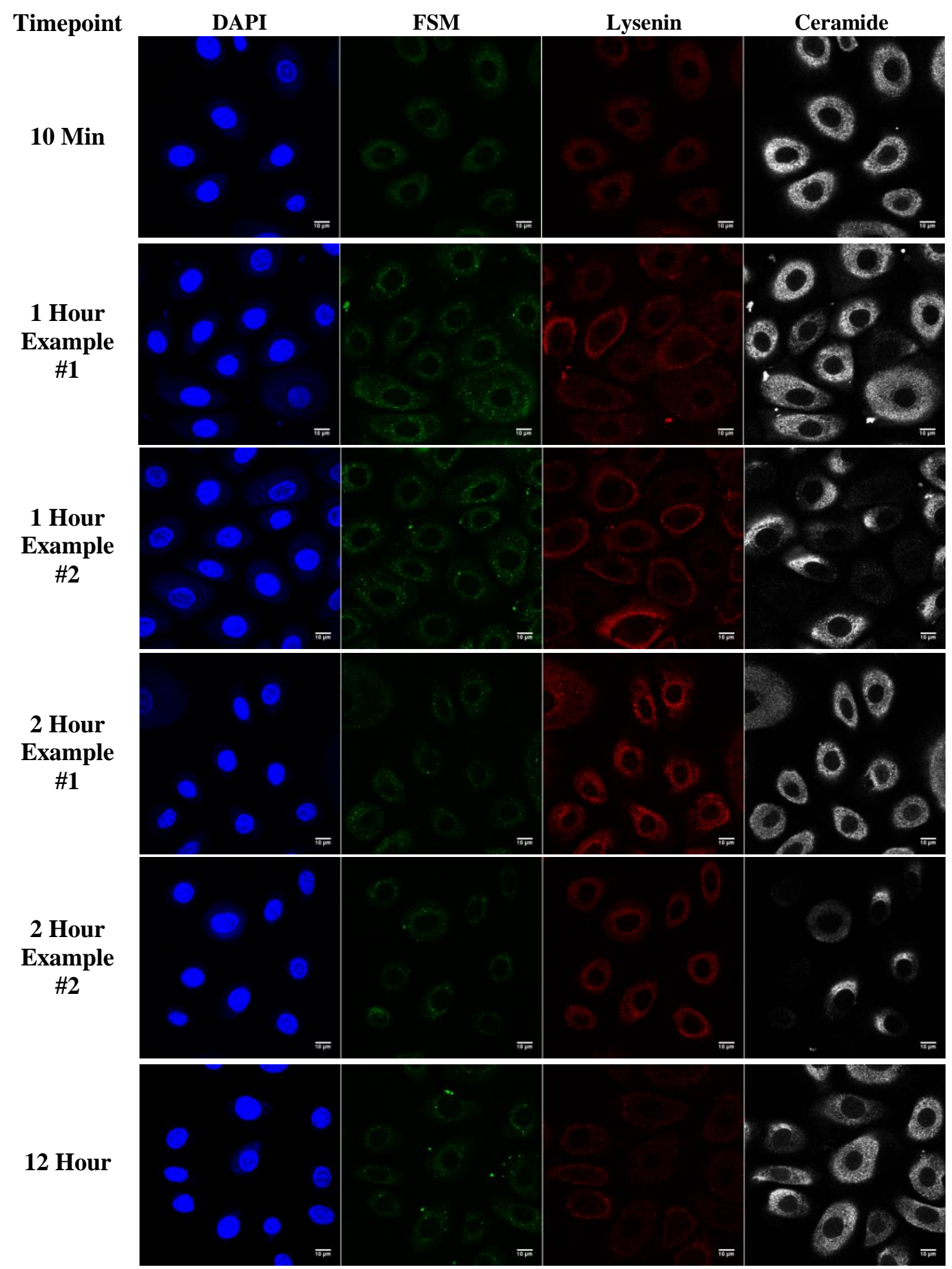

Figure 54: Representative Dual Immunofluorescence 2D Images for Various Continuous FSM Incubation Times. 100x objective.

Two 1 hour and 2 hour examples are provided to demonstrate that the inconsistent ceramide staining made fluorescence distribution quantification difficult. Because the ceramide 
channel was used to generate the ROI for the program quantification, uneven ceramide cytoplasmic staining means the automated macro cannot reliably outline the cell, thus introducing potential bias by only outlining and quantifying cells with a more even cytoplasmic ceramide fluorescence.

Figures 55 and 56 include fluorescence localization results generated from the 2D fluorescence distribution program. The results for FSM, lysenin, and ceramide after different continuous incubation times are presented in separate graphs. Comparisons between stains (in terms of brightness) cannot be made because the signal intensity for one stain (i.e. FSM) is not directly related or proportional to the signal intensity of another stain (i.e. ceramide). This is due to a variety of factors including different dilutions of secondary antibodies were used for immunofluorescence. It is interesting to note that a peak close to the plasma membrane was observed in the 1 hour (Figure 55B black arrow). This correlates with the 1 hour images which displayed a bright plasma membrane staining (Figure 54). Furthermore, the 2 hour lysenin timepoint fluorescence distribution curve exhibited a spike in fluorescence close to the nucleus (Figure 55B red arrow). The data also appears to correlate with images (Figure 54). No other differences in fluorescence distribution curves for FSM, lysenin, and ceramide were observed. 

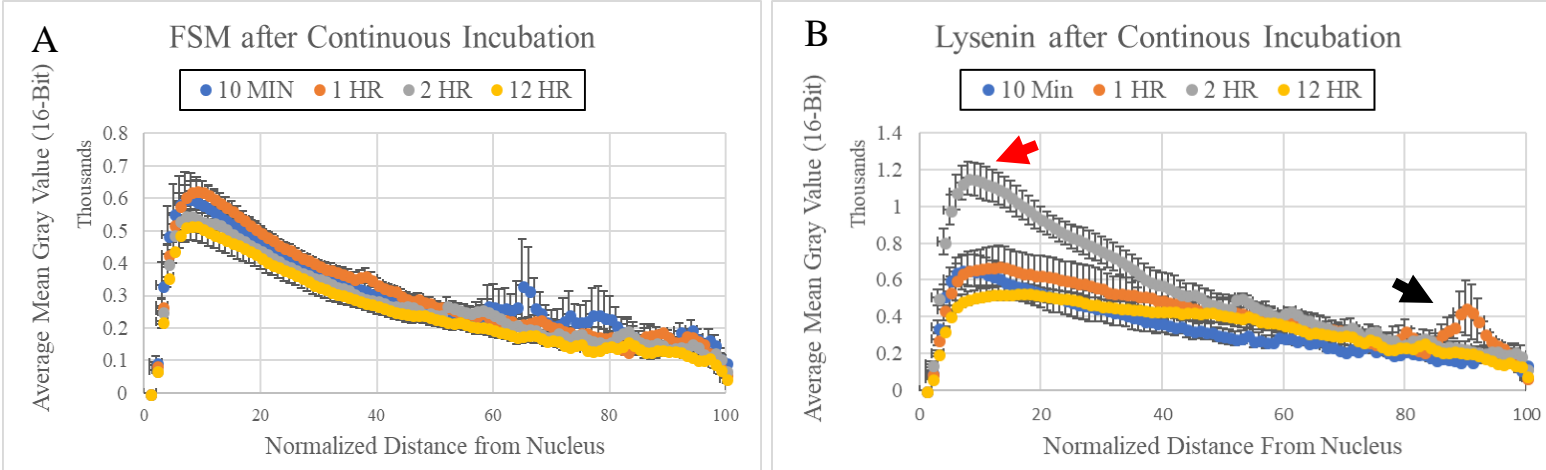

C Ceramide after Continous Incubation

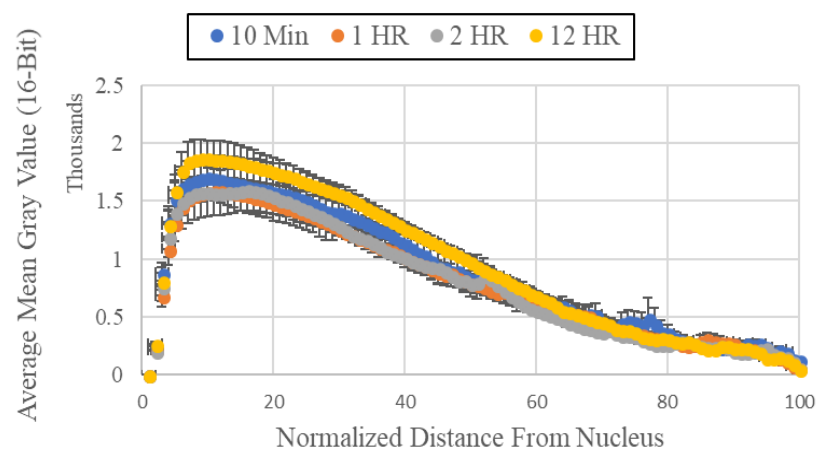

Figure 55: FSM, Lysenin, and Ceramide Fluorescence Distributions over Variable Continuous Incubation Times ( $\mathbf{n}=4$ wells). (A) FSM, (B) Lysenin, (C) Ceramide.

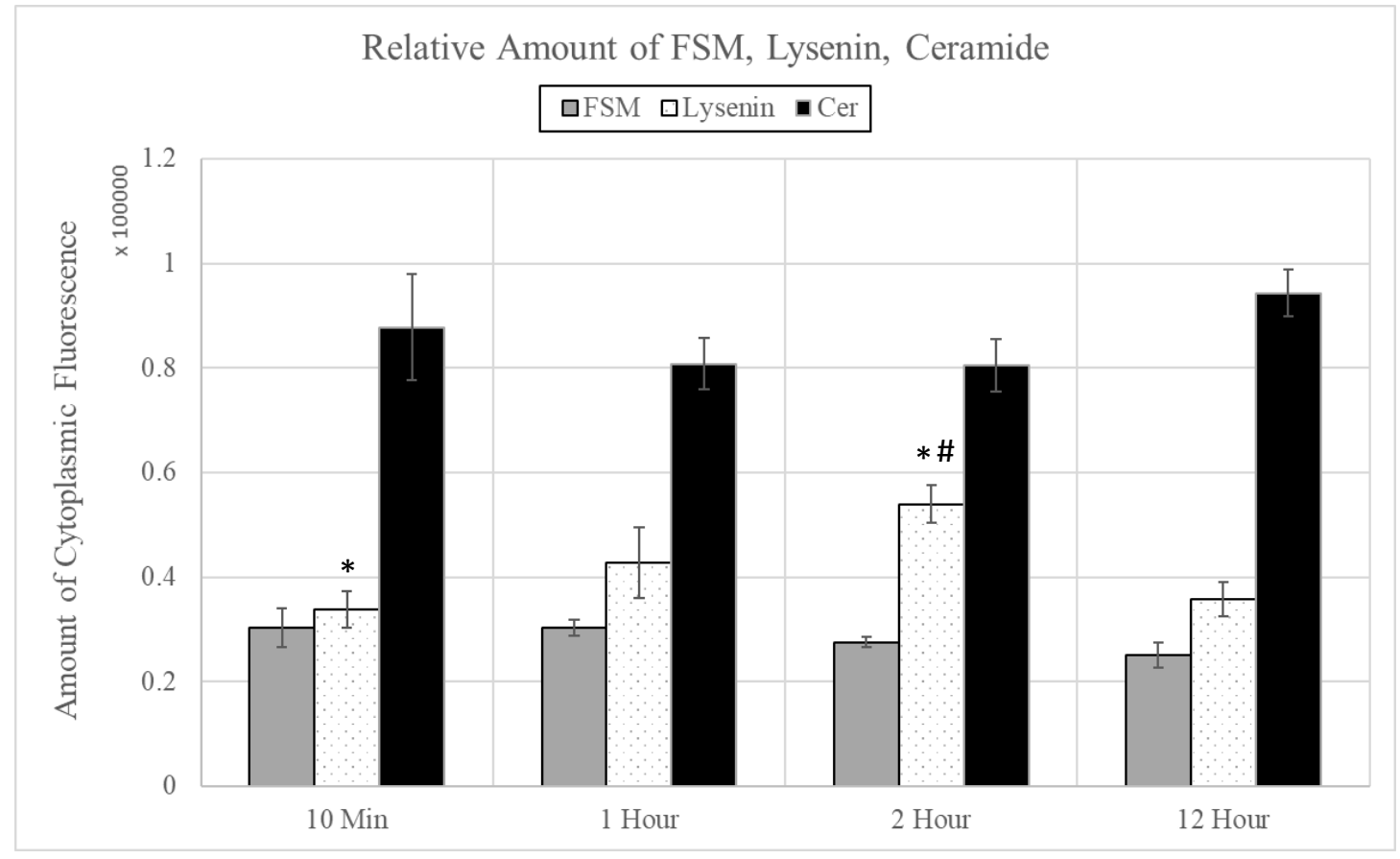

Figure 56: Relative Amounts of Ceramide, FSM, and Lysenin. No significant differences among ceramide and FSM timepoints. \# significant difference from global mean. * significant difference from each other. Standard error bars are represented. 
Tukey HSD pairwise comparisons were only employed for comparisons within each stain (Figure 56). Appendix B includes results of statistical assumptions. No significant differences were observed among FSM and ceramide timepoints. However, for the lysenin stain, the 2 hour (M 53.9k, SE 3.64k summative mean gray value) was significantly greater than the global mean (M 41.53k, SE 4.3k) (p 0.0083 increase of 30\%) and 10 minutes (M 33.7k, SE 3.51k) (p 0.0366 increase of $60 \%$ ).

\subsubsection{Discussion}

After immunofluorescence staining and trafficking analysis, results demonstrated that no significant differences existed within the individual ceramide and FSM stains for 10 min, 1 hour, 2 hour and 12 hour incubation timepoints. Furthermore, FSM data mirrored results from the prior FSM trafficking experiment, even though signal degradation was observed. Within the lysenin stain, the 2 hour was significantly greater than the 10 minute incubation ( $60 \%$ increase). The fluorescence distribution graphs for lysenin also mirrored the intense plasma membrane staining in the 1 hour and predominant intracellular fluorescence in the 2 hour.

Despite the significant difference between lysenin 2 hour and 10 minutes, the biological significance is unknown and could be attributed to measurement error or variability within the system. The lysenin fluctuations suggest endogenous SM levels are changing in a cell, but differences in FSM intensity alone were not detectable with the current analysis method. The inability to detect significant differences in the ceramide stain could also be a result of heterogeneous staining that biased or diluted observable differences. The variability was most apparent in the 24 hour and control plates making fluorescence distribution analysis impossible. More replicates are needed to obtain scientifically relevant data.

Future steps include troubleshooting the reason for inconsistent staining, and either developing a novel analysis technique to reduce bias or analyzing diffuse and patchy ceramide 
cells separately. Another improvement is analyzing fluorescence distribution in 3D to account for signal above/below the nucleus. Finally, more experiments are needed to isolate the cause for the FSM signal degradation. Although Tween-20 was removed from all washes and blocking buffers, degradation was still observed, which suggests the problem is multifaceted and dependent on multiple variables. Instead of quantifying dual immunofluorescence images, the colocalization analysis could be implemented in FSM cells stained either with lysenin and ceramide individually; taking a step back reduces variability and simplifies the complex staining protocol.

Before developing an accurate colocalization method, the stains need to be further optimized to reduce variability within ceramide and isolate the cause for FSM degradation. Overall, the experiment is promising and demonstrates that dual immunostaining is possible, with more optimization in the future. Eventually, the improved staining protocol could be applied to irradiated cells in order to assess post-UV lysenin and ceramide fluctuations. 


\subsection{Spheroid Trial 1: KRTs and 3T3 Single Culture in Non-Adherent U-Wells}

\subsubsection{Summary and Objectives}

Spheroids prove to be a novel method of 3D culture in order to recapitulate the dynamics of cell behavior in a microenvironment similar to tissue [69]-[77]. One goal within the Cal Poly Bio Imaging Lab is to generate a 3D model of physiologically relevant skin to test the BSM and FSM lipid treatments and assess UV photoprotection. Spheroids could potentially serve as one solution but require extensive testing to demonstrate the model is physiologically accurate and equivalent to current 2D monolayers employed in the lab. In general, spheroids can form in a variety of methods with one being non-adherent static culture [69]-[77]. A binding agent, such as methylcellulose, is introduced to the cell suspension and mixed before being dispensed into nonadherent 96-well plates [69]-[77]. The well culture surface prevents cell attachment while the methylcellulose aids in binding among the cells [69]-[77]. After several days of culture, spheroid formation is usually visible under the whitelight microscope, then various treatment conditions (i.e. Hoechst nuclei staining and imaging) can be performed on each individual spheroid.

For this thesis, several experiments were conducted on spheroids, the first being proof-ofconcept to assess the ability of 3T3 and KRT cells to form single-culture spheroids in nonadherent culture conditions. 3T3 cells were chosen as a cell type because they were readily available in lab, possess robust growth characteristics, and proliferate rapidly as an immortalized cell line. Both $3 \mathrm{~T} 3$ and KRT cells were cultured under normal conditions, then trypsinized and suspended in a $2 \% \mathrm{v} / \mathrm{v}$ methylcellulose suspension diluted in 3T3 or KRT media. The appropriate cell number was suspended in the methylcellulose media to ensure approximately 1000 cells were seeded per spheroid. $100 \mu \mathrm{L}$ of cell suspension was pipetted into each well of the 96 -well plate (at a seeding density of 1000 cells per spheroid). Spheroids were allowed to grow in their nonadherent condition for about 5 days and morphology was assessed visually under the whitelight 
microscope every two days. At the end of the 5 day culture, each spheroid was aspirated with a $1000 \mu \mathrm{L}$ pipet tip, then placed into a conical tube; about three spheroids were added to each tube. Spheroids were gently washed once with PBS, fixed for 20 minutes with 3.75\% PFA, then stained with $0.1 \%$ Hoechst diluted in PBS for 20 minutes. The working volume of the solutions added to each conical was $200 \mu \mathrm{L}$. Finally, spheroids were washed three times with PBS, then pipetted into 8-well glass coverslip plates for imaging. Spheroids were located on the confocal then z-stacks with a $1 \mu \mathrm{m}$ step size were obtained from the start of the spheroid (at the glass coverslip interface) to the limit of working distance for the confocal.

\subsubsection{Results}

After two days of culture, spheroids in non-adherent $U$ wells were inspected under the whitelight microscope. While 3T3 spheroids formed a definitive spherical shape (Figure 57A), KRT spheroids were not visible and only small, less distinct clusters of KRTs were observed (black arrows in Figure 57B).
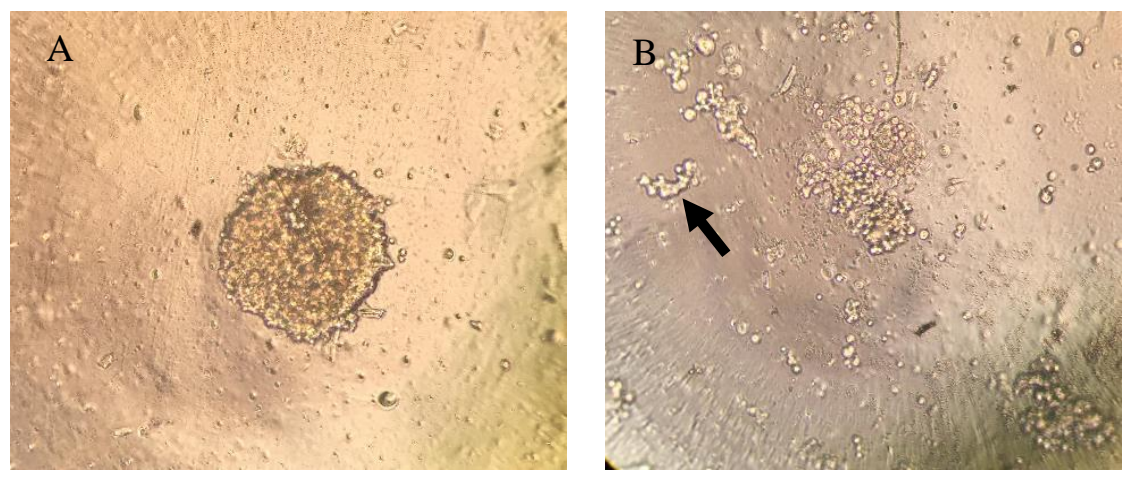

Figure 57: Whitelight Images of 3 T3 and KRT Spheroids after 2 Days for Culture. (A) 3T3 cells formed a distinct spheroid. (B) KRT cells failed to form spheroids and instead small clusters of cells were visible (e.g. black arrow).

Figure 58 includes z-projections of the confocal z-stacks for a representative KRT spheroid (Figure 58A) and 3T3 spheroid (Figure 58B). The confocal z-stacks mirrored the whitelight images 
in Figure 57. Only small aggregates of KRTs were observed while a distinct spheroid (with diameter of approximately $200 \mu \mathrm{m}$ ) formed in the $3 \mathrm{~T} 3$ culture.
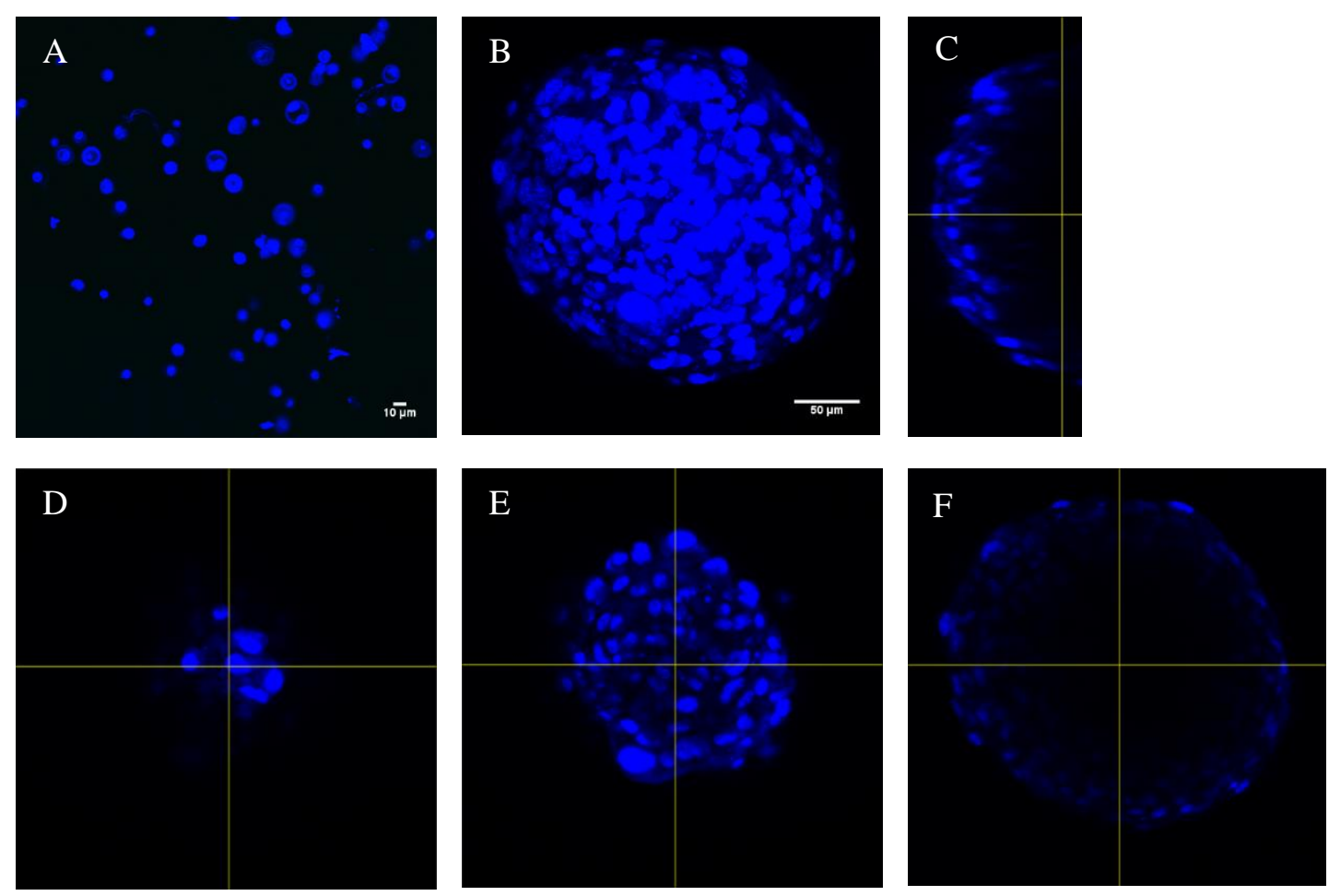

Figure 58: Representative Images of KRT and 3T3 Spheroids. (A) KRT cells failed to form spheroids. (B) 3T3 spheroid. (C) Orthogonal view of 3T3 spheroid showing that the Hoechst dye did not penetrate into the depth of the spheroid. (D) 2D image of start of z-stack, (E) 2D image of middle of z-stack, (F) 2D image of end of z-stack demonstrating the lack of Hoechst dye visibility at the interior core of the spheroid. 40x objective.

Due to the thickness of the spheroid and limited working distance of the 40x confocal objective, the entire spheroid was not able to be imaged. Figure $58 \mathrm{C}$ shows an orthogonal view to demonstrate that only the tip of the spheroid was imaged. As the z-stack depth increased, the intensity of the Hoechst stain diminished. Figures 58D-F include representative 2D images of the spheroid during z-stack sectioning. At the start of the spheroid, bright fluorescence is visible in the middle of the frame (Figure 58D), then progressively radiates outward as the scan depth increases (Figures 58E-F). 
Another observation during imaging was that the Hoechst dye failed to penetrate into the interior of the spheroid. In Figure 58F, a clear lack of Hoechst dye was observed in the center.

\subsubsection{Discussion}

3T3s, but not KRTs, were able to form robust spheroids, and confocal imaging demonstrated that the Hoechst dye did not penetrate sufficiently into the interior of the spheroid. In order to encourage KRTs to form spheroids, it was hypothesized that fibroblasts were needed to facilitate adhesion. The concentration of methylcellulose and seeding density (among other factors) could also affect spheroid formation. Therefore, in the subsequent experiment, co-culture spheroids of 3T3s and KRTs were cultivated for different seeding densities and methylcellulose concentrations. 


\subsection{Spheroid Trial 2: KRT and 3T3 Dual Culture}

\subsubsection{Summary and Objectives}

The goal of the following experiment was to assess whether culturing KRTs with 3T3s facilitated spheroid formation. Multiple parameters (methylcellulose concentration, total cells per spheroid, and seeding density ratios) were varied as indicated in Table XVI. Note that the media composition ratio matched the seeding density ratio (i.e. if cells were mixed $1: 1$, the media for the spheroid was also mixed 1:1). In order to distinguish the KRTs from 3T3s, CellTracker Red was incubated with the KRTs to stain their cytoplasm. All cells were stained with Hoechst.

Table XVI: Experimental Parameters Varied for Dual 3T3 KRT Spheroids

\begin{tabular}{|c|c|}
\hline Experimental Parameter & Value \\
\hline Methylcellulose Concentration & $20 \% \mathrm{v} / \mathrm{v}$ \\
& $40 \% \mathrm{v} / \mathrm{v}$ \\
\hline Total Cells & 1,000 \\
& 5,000 \\
\hline Seeding Densities Ratio (3T3:KRT) & $1: 1$ \\
& $2: 1$ \\
& $1: 2$ \\
\hline Media Composition Ratio (3T3 media: KRT & $1: 1$ \\
media) Matched seeding density ratio & $2: 1$ \\
& $1: 2$ \\
\hline Culture Time & 5 Days \\
\hline
\end{tabular}

\subsubsection{Results}

Visual inspection under the whiltelight microscope after 3 days of culture suggested that spheroids formed in the dual culture cases with 3T3s and KRTs (Figure 59A\&B). No spheroids were generated in the KRT only culture (Figure 59C). Furthermore, in the dual culture, some satellite colonies or small aggregates of cells formed around the main spheroid (black arrow). 

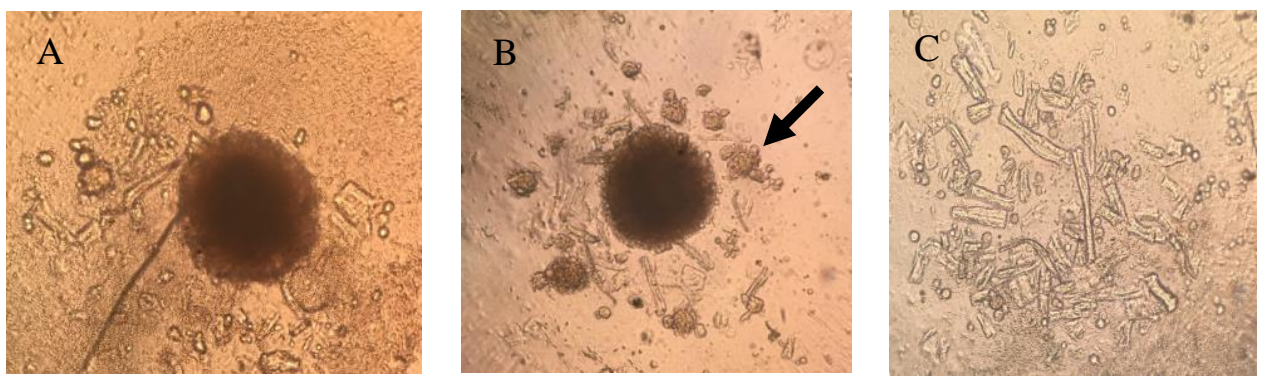

Figure 59: Whitelight Images after 3 Days of Culture. (A) 2:1 40\%. (B) 1:1 40\%. (C) KRT only $40 \%$. Satellite colonies of KRTs are observed surrounding the main spheroid (arrow). No spheroids formed in the KRT only culture.

Figure 60 includes representative images of dual spheroids of different parameters. The bright red fluorescence with blue nuclei stain corresponded to KRTs stained with CellTracker red cytoplasmic stain. Hoechst nuclei without CellTracker staining distinguished 3T3 from KRT cells. The representative images demonstrate that KRTs were present in the spheroids, but the degree to which they integrated into the spheroid varied. In most cases, the KRTs did not evenly distribute and instead formed satellite colonies (as demonstrated by the intense red fluorescence clusters). 


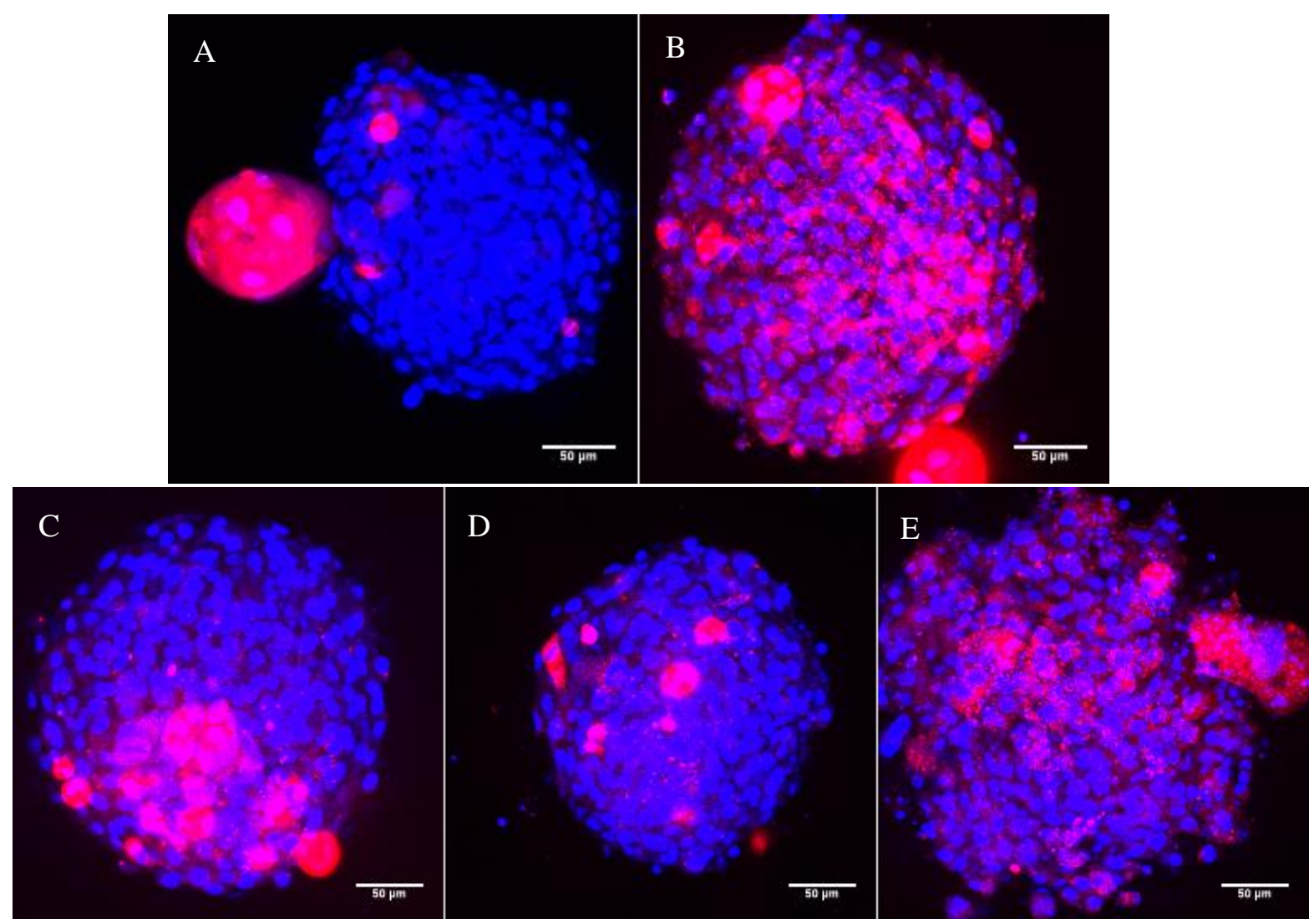

Figure 60: Z-Projections of Confocal Spheroid Z-Stacks. (A) 3T3: KRT at 1:1 ratio with 1,000 cells total and 40\% methylcellulose (B) 3T3: KRT at 1:1 ratio with 5,000 cells total and 40\%, methylcellulose (C) 3T3: KRT at 2:1 ratio with 5,000 cells and 20\% methylcellulose (D) 3T3: KRT at 2:1 ratio with 5,000 cells total and 40\%, methylcellulose (E) 3T3: KRT at 1:2 ratio with 5,000 cells total and $40 \%$ methylcellulose. $40 \mathrm{x}$ objective.

\subsubsection{Discussion}

The dual spheroid experiment suggests that 3T3 fibroblasts are needed to encourage KRT spheroid formation. However, KRT integration was limited and satellite colonies were observed adjacent to the main $3 \mathrm{~T} 3$ spheroid. Future steps include repeating this study and investigating other factors to encourage full KRT incorporation into spheroids. Furthermore, using a more relevant dermal fibroblast model could potentially encourage KRT spheroid formation. 


\subsection{Spheroid Trial 3: Culture with Agarose-coated Flat Bottom Wells with Triton $X$ and FSM Incorporation}

\subsubsection{Summary and Objectives}

The main goals for this final trial were three-fold. Table XVII summarizes the experimental parameters used for this third experimental trial. First, flat bottom wells were coated with agarose to substitute for the more expensive Corning® non-adherent U-well utilized in past experiments. 96-well flat bottom plates were coated with $200 \mu \mathrm{L}$ to form a meniscus and nonadherent surface. Approximately 1,000 3T3 cells suspended in 20\% v/v methylcellulose were seeded per 8-well plate and incubated for 5 days.

The second goal was proof-of-concept to determine if FSM fluorescence would be present after incubation with the spheroids. 5uM FSM solution was introduced to 3T3 spheroids for 1 hour and 24 hours. Immediately after the incubation, the spheroids were fixed and prepped for subsequent staining.

Finally, the third goal was to assess whether Triton X permeabilization could encourage Hoechst penetration into spheroids. Half of the $3 \mathrm{~T} 3$ spheroids were incubated with $0.1 \%$ Triton $\mathrm{X}$ for 20 minutes, then all spheroids were stained with $0.1 \%$ Hoechst for 24 hours.

\section{Table XVII: Spheroid Trial 3 Experimental Parameters}

\begin{tabular}{|c|c|}
\hline Methylcellulose Concentration & $\mathbf{2 0 \%}$ \\
\hline Total Cells & 750-1000 cells/spheroid \\
\hline Culture Time & 5 Days \\
\hline Treatment & 5uM FSM for 1 hour or 24 hours \\
\hline Permeabilization & $\begin{array}{c}0.1 \% \text { Triton X 20 minutes } \\
\text { No Triton X }\end{array}$ \\
\hline Staining & $0.1 \%$ Hoechst for 24 hours \\
\hline
\end{tabular}




\subsubsection{Results}

Figure 61 includes white light images of 3T3-methylcellulose solutions seeded onto agarose coated wells (Figure 61A) and non-agarose coated wells (Figure 61B). Spheroid formation under agarose conditions was successful. Agarose coating formed a concave meniscus and simulated the U-well shape of the previously used Corning® plates (Figure 61C).
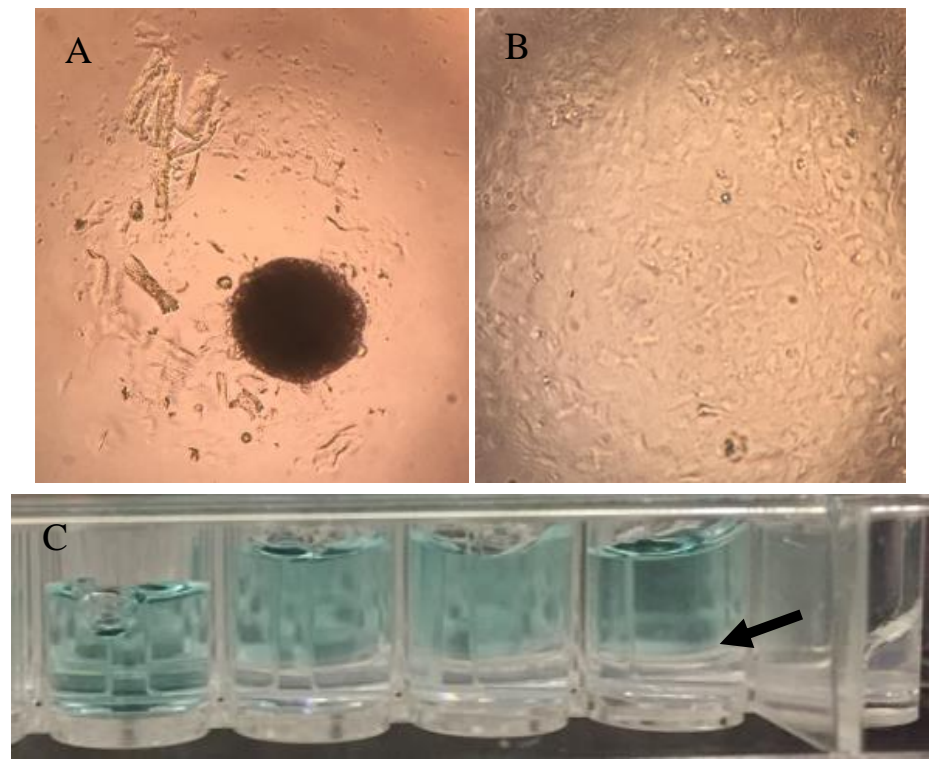

Figure 61: Agarose is Necessary and Sufficient to Create a Non-Adherent Environment for Spheroid Formation of 3 T3 Cells. (A) Agarose, (B) No Agarose, (C) Agarose Meniscus (black arrow)

Finally, Figure 62 includes representative images of the spheroids incubated with FSM then permeabilized with Triton X prior to Hoechst staining. Even with permeabilization, Hoechst did not penetrate the interior of the spheroids (Figure $62 \mathrm{C} \& \mathrm{E}$ ). Furthermore, the FSM fluorescence was only observed along the top of the spheroid, independent of the incubation time and Triton X incubation (Figure $62 \mathrm{~F}, \mathrm{G}, \mathrm{H}$ ). 

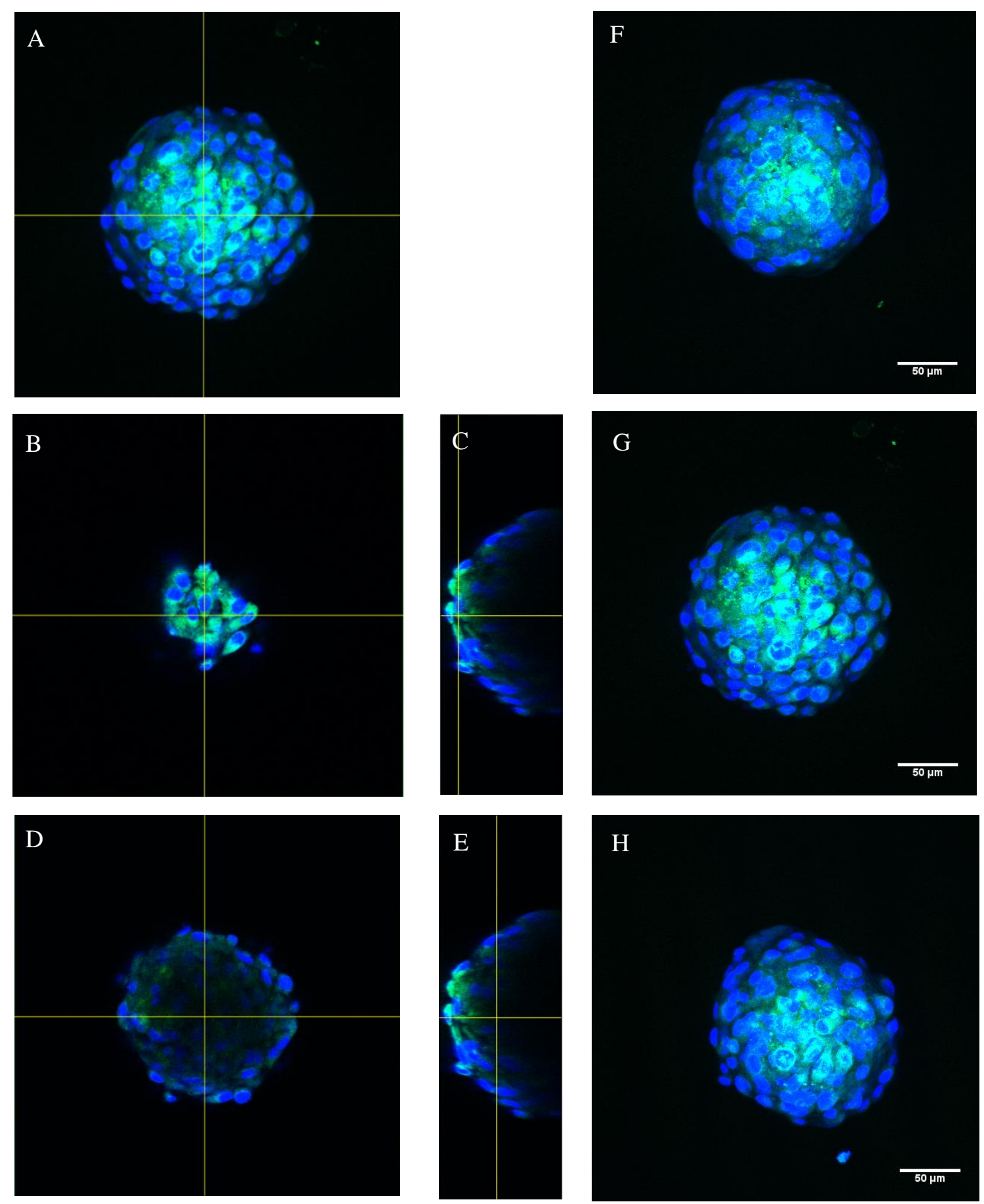

Figure 62: Orthogonal View of 3D Spheroid with FSM and Triton X. (A) Representative FSM spheroid z-project. (B) Start of z-stack for representative FSM spheroid. (C) Corresponding orthogonal view. (D) Middle of z-stack for representative FSM spheroid (E) Corresponding orthogonal view. (F) FSM 1 hour, no Triton X (G) FSM 24 hours, no Triton X (H) FSM 24 hours, Triton X. 40x objective. 


\subsubsection{Discussion}

Results from this final experiment suggest that agarose coating is a sufficient alternative to Corning ${ }^{\circledR}$ U-well plates in terms of promoting $3 \mathrm{~T} 3$ spheroid formation. Furthermore, agarose provides a more cost-effective alternative.

Unfortunately, Triton $\mathrm{X}$ incubation did not mitigate the Hoechst penetration insufficiencies. FSM also failed to penetrate deep into the spheroid and instead collected at the top of the spheroid regardless of incubation duration. Future steps include running more studies on the agarose coated wells and investigating other factors responsible for the lack of Hoechst and FSM penetration into the interior of the spheroids. 


\subsection{Spheroid Quantification: Dye Penetration}

\subsubsection{Summary and Objectives}

For the final spheroid experiment, a method was developed to quantify dye penetration into 3D spheroids. This analysis method could potentially be used for future studies into FSM dye penetration.

\subsubsection{Results}

Figure 63 includes multiple images of the process for spheroid depth of penetration analysis. Each image in the z-stack was thresholded to remove slices without a fluorescence signal (Figure $63 \mathrm{~A} \& \mathrm{~B}$ ). A projection of the entire z-stack was used to outline the spheroid (Figure $63 \mathrm{C}$ ). Using the spheroid outline, the 2D fluorescence distribution macro was run on slices corresponding to the start, $1 / 10,2 / 10,3 / 10,4 / 10,5 / 10,6 / 10,7 / 10,8 / 10,9 / 10$, and end of the z-stack. Figures 63D-F show representative images of divisions 1/10, 3/10, and 8/10. Figure $63 \mathrm{G}$ is a graphical representation of the fluorescence distributions for each division. Note that divisions $2 / 10$ and $3 / 10$ have bright staining close to the center, then the fluorescence moves outward towards the end of the outline as the depth increases. Finally, Figure 64 shows results of the CUM TRAPZ area under the curve calculations for each image division across all treatments. The amount of fluorescence peaks at 2/10 divisions then gradually drops as the depth increases. 

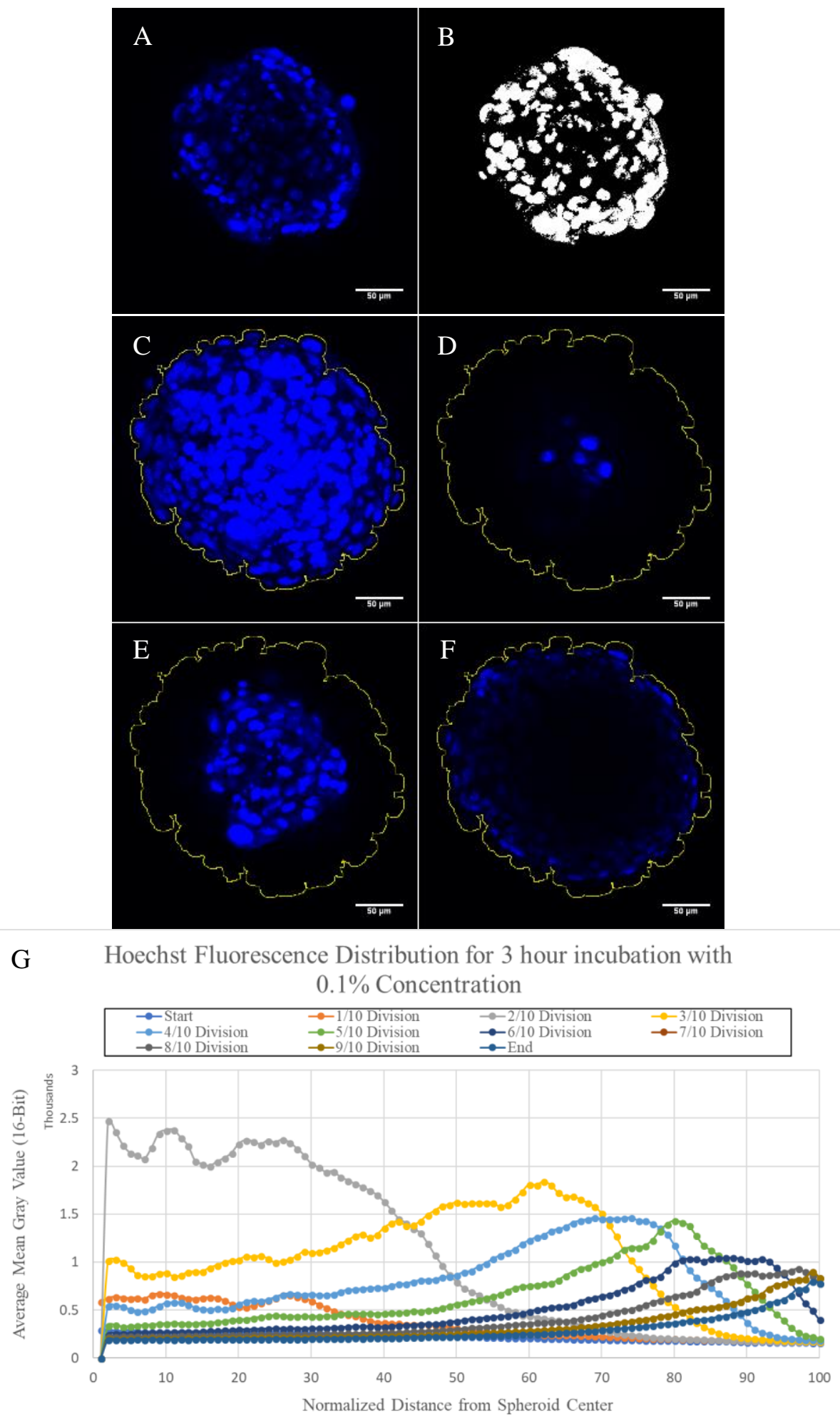

Figure 63: Analysis Method for Spheroids. (A) Slice in Spheroid, (B) Binary, (C) Z-projection with outlines, (D) 1/10 division, (E) 3/10 division, (F) 8/10 division. (G) Graphical representation of the fluorescence distribution. 


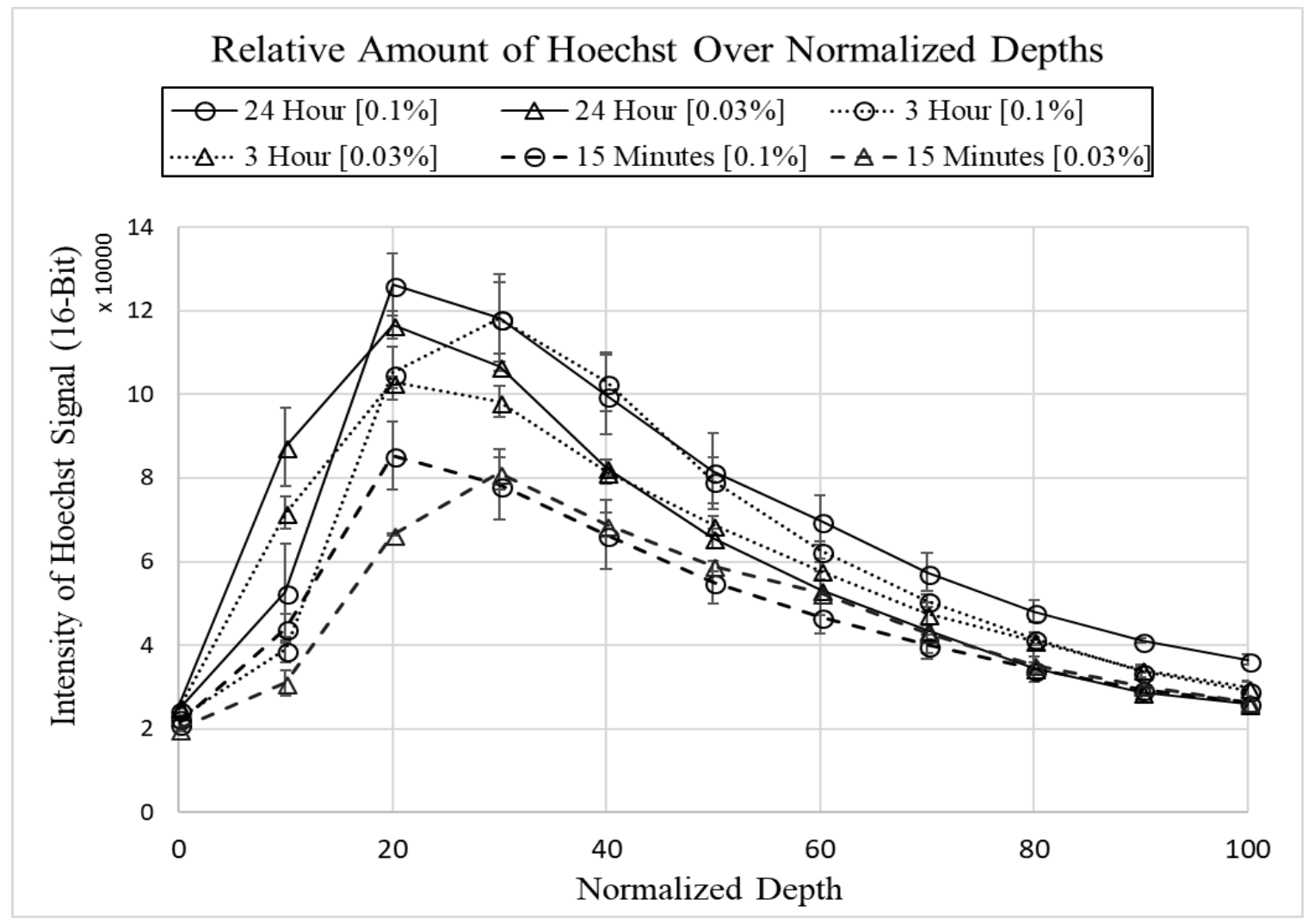

Figure 64: Relative Amount of Hoechst Dye over Normalized Spheroid Depth. Standard error bars are represented.

Despite a high concentration of Hoechst and 24 hour incubation, the dye failed to penetrate the interior of the spheroid. Nevertheless, the quantification method can be used to assess future FSM trafficking in a 3D spheroid model.

\subsubsection{Discussion}

The described image analysis method provides a way to analyze FSM and Hoechst dye penetration into spheroids for future work. The data matches visual results from the spheroid image over varying z-stack depths and demonstrates quantitatively that Hoechst insufficiently penetrates the spheroid core independent of incubation time and dye concentration.

The results could potentially be a confocal imaging limitation, and it is unknown whether the result is an artifact of insufficient imaging. For example, the confocal objective working 
distance is not appropriate or the spheroid is too thick to allow sufficient laser penetration for imaging. Furthermore, it is unknown if cells are even present in the center, or if a necrotic core formed in each spheroid due to insufficient nutrient diffusion (thus suggesting a reason for the lack of nuclear staining). Future steps include using multiphoton and conducting histology to physically section through the spheroids and stain them. 


\section{DISCUSSION AND CONCLUSIONS}

The following describes the three main goals, implications, limitations, and future steps from the completed experiments.

\section{Goal 1: Does FSM intracellular trafficking change with varying incubation times, and after $U V B$ radiation?}

a. Subset 1: Characterize FSM in KRT culture during live and fixed cell cultures using fluorescence confocal imaging

b. Subset 2: Assess the localization of FSM in normal and UVB-stressed KRTs under varying incubation times

c. Subset 3: Demonstrate functional equivalence of bovine milk SM to its fluorescent counterpart

The application of Goal 1 was to determine an optimal incubation time by studying FSM trafficking differences after various continuous incubation times and after UV radiation. First, the functionality of the $5 \mu \mathrm{M}$ Fluorescent Sphingomyelin (FSM) concentration in a live and fixed Keratinocyte (KRT) skin cell monolayer using confocal fluorescence microscopy was demonstrated. Results indicated that a $5 \mu \mathrm{M}$ FSM incubation yielded robust fluorescence and was able to undergo fixation prior to imaging. Blebbing and both plasma membrane intracellular staining was observed, consistent with literature [63]. A higher magnification, like the 100X objective, was deemed more suitable for higher resolution intracellular imaging. Subsequently, FSM was incubated for various timepoints at $37^{\circ} \mathrm{C}$ and imaged live. Images suggested that FSM intensity and location does not change significantly with incubation time over 24 hours. Although live imaging is advantageous for time studies, without the proper equipment to maintain physiologic temperatures, cell phenotype and FSM trafficking can be affected. In addition, FSM photobleaching and cell movement was also deemed problematic, necessitating future fixed 
experiments for FSM trafficking quantification. Furthermore, cells were incubated with FSM at room temperature to demonstrate the FSM endocytic and exocytic temperature dependence and demonstrate that temperature, along with many other extraneous variables, need to be controlled in experiments.

To obtain quantifiable data, two experiments were conducted. First, cells were incubated with FSM for 10 minutes, 30 minutes, 1 hour, 2 hour, 3 hour, 4 hour, 12 hour, and 24 hour, fixed in $3.75 \%$ PFA, imaged, then analyzed with a fluorescence distribution macro developed previously in the research lab. Area under the curve data mirrored the live time study and demonstrated that intracellular FSM trafficking was independent of the continuous incubation time.

Second, cells were continuously incubated with FSM for 1 hour or 24 hours, subjected to UV radiation, and fixed at different timepoints after UV. Area under the fluorescence distribution curves demonstrated no difference among the 24 hour incubation post-UV timepoints, but some slight variation for the 1 hour incubation timepoints. In particular, the 12 hour post-UV area under the curve was significantly greater than 1 hour and 2 hour by approximately $50 \%$ and $41 \%$ respectively. In addition, the no UV control was significantly lower ( $24 \%$ decrease) compared to the 24 hour post-UV timepoint (24\% decrease) and 12 hour post-UV timepoint $(49.29 \%$ decrease). Across all post-UV timepoints, the 1 hour incubation had a consistently higher average cytoplasmic mean gray value compared to the 24 hour incubation, with statistical significance for 12 hour and 24 hour post-UV.

The results potentially point towards a shorter timepoint for BSM/FSM incubation prior to UV (1 hour) and warrant further investigation into the photoprotective effects of a shorter incubation timepoint. Replication and a larger sample size are both necessary before making definitive conclusions. In the future, use of 1-well plates (instead of 8-wells) may prove advantageous to provide a more consistent UV dosage without the plastic division reflecting and 
obstructing UV light. Confocal images could be taken in the center of the well and repeated for multiple plates, where each plate is treated as an independent unit (instead of each well, as in the case of this thesis). Replicating with multiple 1-well plates per treatment will ensure complete independence among treatments and an even UV dosage.

Finally, FSM was deemed functionally equivalent to BSM in terms of p21 photoprotection. FSM decreased p21 levels by $21.6 \%$ compared to the no FSM control. Similarly, BSM decreased p21 levels by $51 \%$ compared to the control, which was statistically significant. The lack of significance could be due to concentration mismatch between the FSM $5 \mu \mathrm{M}$ and $0.1 \% \mathrm{BSM}$ (a $1.3 \mathrm{mM}$ concentration). In the future, the concentration of FSM should be optimized by diluting the $1 \mathrm{mM}$ stock to yield incremental concentrations of FSM, exposing to cells for a consistent incubation time, quantifying dosage-dependent p21 effects, then selecting the concentration that offers the most photoprotection.

The FSM trafficking studies are limited in that a low sample size $(n=4$ or 6$)$ was used for both quantification experiments. The 2D fluorescence distribution analysis could potentially introduce bias by improperly drawing cell ROIs. In addition, fluctuations in the amount of FSM added, the positioning of the plate under the UV lamp, or changes in the day-to-day confocal laser characteristics could all affect data. Additionally, harsh washing of cells before fixation could remove weakly adherent and damaged cells, thereby skewing data. Finally, it is unknown whether the measurement system is actually sensitive enough to detect small significant differences in brightness, and furthermore, what a difference in fluorescence means biologically.

Future work includes more replication, using 1-well plates for experiments, refining the 2D fluorescence distribution analysis, extending analysis to 3D images, reducing the severity of wash steps, and implementing various controls. First, a SMase enzyme, known to degrade SM, could be introduced to cells loaded with FSM as a positive control to validate the analysis system. Subsequent images/analysis could demonstrate how/if FSM intensity degrades after the SMase 
enzyme, and if the current 2D image analysis is sensitive to detect a difference. The SMase control will help isolate problems with the current analysis. Furthermore, experiments could be validated with HPTLC, a traditional technique to quantify lipid content. Finally, a 3D analysis system, similar to the MABLAB code developed by Pike et al., could be used to obtain more accurate data and encompass FSM plasma membrane expression surrounding the cell [86]. Replication, tight control of extraneous variables, SMase controls, validation with HPTLC, and 3D image analysis are all necessary future steps.

Goal 2: Do endogenous SM and ceramide levels fluctuate with exogenous FSM incubations? Is it possible to characterize, with fluorescence microscopy, the identity of the observed FSM signal over time?

a. Subset 1: Develop immunofluorescence staining protocols for both endogenous SM (lysenin) and endogenous ceramide

b. Subset 2: Characterize how endogenous SM and ceramide levels change with varying FSM incubation timepoints

For goal 2, immunofluorescence stains were optimized for both endogenous sphingomyelin (lysenin) and ceramide in order to reduce non-specific binding. The staining parameters were then implemented in a dual staining protocol with FSM, lysenin, and ceramide staining to quantify $2 \mathrm{D}$ trafficking of the respective stains. Results demonstrated that no significant differences existed within the individual ceramide and FSM stains for $10 \mathrm{~min}, 1$ hour, 2 hour and 12 hour incubation timepoints. However, within the lysenin stain, the 2 hour was significantly greater than the 10 minute incubation (60\% increase). Despite this difference, the biological significance is unknown and could be attributed to the aforementioned measurement and imaging errors. The data could also indicate homeostatic fluctuations in lysenin levels not visible with just FSM staining alone. However, without validating the sensitivity of the measurement system with a positive aSMase control, conclusions about biological significance 
cannot be drawn. The results demonstrate that dual immunofluorescence staining is possible and the images are appropriate for future colocalization experiments to better characterize FSM metabolism over time.

Future steps include troubleshooting the reason for inconsistent staining and refining the analysis technique because further optimization is needed before quantifying colocalization of FSM with lysenin and FSM with ceramide. Senescence or proliferation markers could be correlated with lysenin and ceramide to assess differences in the state of the cell. In addition, adopting the previously mentioned MATLAB 3D fluorescence distribution code could account for differences in the observed heterogeneous ceramide and lysenin staining [86]. Finally, lysenin could potentially serve as a fluorescence marker for BSM localization in a cell. Studies similar to FSM could be run in parallel to assess differences in localization between the two SM analogues.

\section{Goal 3: Are cell spheroids a viable platform to move future research from a $2 D$ to $3 D$ model?}

a. Subset 1: Develop a method to culture NIH $3 T 3$ immortalized mouse fibroblast spheroids, for eventual incorporation of KRT cells and FSM

b. Subset 2: Quantify dye penetration over various concentrations and incubation times

The final goal of this thesis was to provide initial results towards development of a 3D KRT spheroid model to better recapitulate the dynamics and microenvironment of tissue. Spheroids were plated with $3 \mathrm{~T} 3$ fibroblasts and mixed with KRTs in non-adherent U-well plates from Corning®. KRTs failed to integrate into the spheroid and instead formed satellite clumps/colonies adjacent to the main spheroid. Agarose coated wells were tested as an alternative to Corning $®$ non-adherent U-wells. 3T3 spheroids successfully formed in the agarose wells, but Hoechst dye failed to penetrate even with Triton X permeabilization. Finally, in the last 
experiment, FSM was introduced to the spheroids, and a method to quantify dye penetration was developed for future studies.

Future work includes developing a more consistent method to handle the spheroids in a high-throughput manner. Histological (either paraffin or frozen embedded) sections of the spheroids can be obtained to definitively characterize the spheroid core. Given the difficulty with handling and sectioning individual spheroids, Gong et al. developed methods for controlling location and growth of spheroids by manufacturing micro-wells [75]. Ivanov et al. micropatterned agarose, cultured spheroids into each individual well, then sealed the wells with agarose before sectioning [76]. Furthermore, Wong et al. embedded spheroids in HistoGel® prior to paraffin embedding and sectioning [74]. High-throughput spheroid handling techniques and assessment methodologies are possible but require optimization.

Furthermore, methods to encourage KRT spheroid incorporation need to be developed. A more relevant dermal fibroblast cell line, instead of 3T3 mouse fibroblasts, should be used to replicate the work of a few recent research groups [79]. In addition, the use of calcium supplements in media could encourage skin cell stratification [16], [79] Spheroids can be treated with the same experimental parameters as the monolayers (including FSM incubation, UV, immunofluorescence staining, and imaging).

Finally, the use of spheroids as an appropriate tissue engineered skin equivalent needs to be validated and justified against gold-standard (flat sheet-based culture) and previous lab methods (2D monolayers). Although $3 \mathrm{~T} 3$ spheroids were successfully generated, more work is needed to demonstrate the utility of the model.

In summary, this thesis aimed to expand on past BSM research by clarifying the mechanism for photoprotection with a fluorescent analogue. FSM was found to yield similar photoprotection as its BSM counterpart, and FSM intracellular fluorescence distributions were 
independent of continuous incubation time. Future investigation into shorter incubation timepoints prior to UV is needed, especially considering the observed post-UV fluctuations in total cellular fluorescence compared to the conventional 24 hour incubation. Furthermore, lysenin and ceramide immunostaining hold promise for further endogenous sphingolipid studies in addition to colocalization analysis. Finally, a 3T3 fibroblast spheroid model demonstrated proofof-concept for more relevant 3D skin models. The goal of future research is to ultimately elucidate the photoprotective potential of sphingomyelin in order to provide a natural alternative to sunscreen and prevent NMSC incidence in the general population. 


\section{BIBLIOGRAPHY}

[1] V. Barton et al., "Nonmelanoma skin cancer and risk of all-cause and cancerrelated mortality: a systematic review," Arch. Dermatol. Res., vol. 309, no. 4, pp. 243-251, 2017.

[2] R. Feehan and L. M. Shantz, "Molecular Signaling Cascades Involved in NonMelanoma Skin Carcinogenesis,” vol. 473, no. 19, pp. 2973-2994, 2016.

[3] S. Kim, S.-G. Jin, and G. P. Pfeifer, "Formation of cyclobutane pyrimidine dimers at dipyrimidines containing 5-hydroxymethylcytosine," Photoche Photobiol., vol. 12, no. 8, pp. 1409-1415, 2013.

[4] R. P. Rastogi, Richa, A. Kumar, M. B. Tyagi, and R. P. Sinha, "Molecular Mechanisms of Ultraviolet Radiation-Induced DNA Damage and Repair," J. Nucleic Acids, vol. 2010, pp. 1-32, 2010.

[5] "Skin Cancer Foundation," 2018. [Online]. Available: https://www.skincancer.org/.

[6] B. Carroll, C. Donaldson, and L. Obeid, "Sphingolipds in the DNA Damage Response," Adv. Biol. Regul., vol. 344, no. 6188, pp. 38-52, 2015.

[7] G. M. Cooper, "Tumor Suppressor Genes Identification of Tumor Suppressor Genes," Natl. Cent. Biotechnol. Inf., pp. 1-7, 2000.

[8] J. T. Zilfou and S. W. Lowe, "Tumor suppressive functions of p53.," Cold Spring Harb. Perspect. Biol., vol. 1, no. 5, pp. 1-12, 2009.

[9] T. Abbas and A. Dutta, "p21 in cancer: intricate networks and multiple activities," Nat. Rev. Cancer, vol. 9, no. 6, pp. 400-414, 2009.

[10] M. Schlumpf, B. Cotton, M. Conscience, V. Haller, B. Steinmann, and W. Lichtensteiger, "In vitro and in vivo estrogenicity of UV screens," Environ. Health Perspect., vol. 109, no. 3, pp. 239-244, 2001.

[11] S. Q. Wang et al., "Safety of Oxybenzone: Putting Numbers Into Perspective," Arch. Dermatol., vol. 147, no. 7, p. 865, 2011.

[12] H. W. Lim, M. I. Arellano-Mendoza, and F. Stengel, "Current challenges in photoprotection,” J. Am. Acad. Dermatol., vol. 76, no. 3, pp. S91-S99, 2017.

[13] K. Campbell, "The Use of Sphingomyelin to Protect Against UV Induced DNA Damage in Human Keratinocytes," 2015.

[14] K. Yague De Guzman, "Protective Effects of Sphingomyelin Against UV Photodamage In Human Keratinocytes," no. December, p. 136, 2013.

[15] OpenStax College, Anatomy \& Physiology. Houston, TX, 2013.

[16] A. Baroni, E. Buommino, V. De Gregorio, E. Ruocco, V. Ruocco, and R. Wolf, "Structure and function of the epidermis related to barrier properties," Clin. 
Dermatol., vol. 30, no. 3, pp. 257-262, 2012.

[17] “Skin Cancer Information - SkinCancer.org," 2018. [Online]. Available: https://www.skincancer.org/skin-cancer-information. [Accessed: 21-Feb-2018].

[18] F. R. De Gruijl and L. H. F. Mullenders, "Ultraviolet Radiation as a Carcinogen," Elsevier, vol. 14, no. March 2017, pp. 168-182, 2018.

[19] E. Tur, Environmental Factors in Skin Diseases, 35th ed. Basel, Switzerland: Karger, 2007.

[20] J. Yokota, "Tumor progression and metastasis," Carcinogenesis, vol. 21, no. 3, pp. 497-503, 2000.

[21] V. V. Shah et al., "Dermatologists' Perceptions, Recommendations, and Use of Sunscreen," JAMA Dermatology, vol. 153, no. 1, p. 97, 2017.

[22] L. A. Lemonnier, D. L. Dillehay, M. J. Vespremi, J. Abrams, E. Brody, and E. M. Schmelz, "Sphingomyelin in the suppression of colon tumors: Prevention versus intervention," Arch. Biochem. Biophys., vol. 419, no. 2, pp. 129-138, 2003.

[23] Y. Haruta-Ono et al., "Orally administered sphingomyelin in bovine milk is incorporated into skin sphingolipids and is involved in the water-holding capacity of hairless mice," J. Dermatol. Sci., vol. 68, no. 1, pp. 56-62, 2012.

[24] A. Charruyer et al., "Decreased ceramide transport protein (CERT) function alters sphingomyelin production following UVB irradiation," J. Biol. Chem., vol. 283, no. 24, pp. 16682-16692, 2008.

[25] Y. Uchida et al., "Hydrolytic pathway protects against ceramide-induced apoptosis in keratinocytes exposed to UVB," J. Invest. Dermatol., vol. 130, no. 10, pp. 2472-2480, 2010.

[26] W. Zhang et al., "UVB-induced apoptosis drives clonal expansion during skin tumor development," Carcinogenesis, vol. 26, no. 1, pp. 249-257, 2005.

[27] A. S. Don, X. Y. Lim, and T. A. Couttas, "Re-configuration of sphingolipid metabolism by oncogenic transformation," Biomolecules, vol. 4, no. 1, pp. 315353, 2014.

[28] S. Paxton, M. Peckham, K. Adele, S. Paxton, K. Adele, and M. Peckham, "The Leeds Histology Guide.” 2003.

[29] K. R. Feingold, "The outer frontier: the importance of lipid metabolism in the skin: Fig. 1.," J. Lipid Res., vol. 50, no. Supplement, pp. S417-S422, 2009.

[30] M. Yaar and B. A. Gilchrest, "Ageing and photoageing of keratinocytes and melanocytes," pp. 583-592, 2001.

[31] T. Hirobe, "Dermatologica Sinica Keratinocytes regulate the function of melanocytes," Dermatologica Sin., vol. 32, no. 4, pp. 200-204, 2014.

[32] A. Sandilands, C. Sutherland, A. D. Irvine, and W. H. I. McLean, "Filaggrin in the 
frontline: role in skin barrier function and disease," J. Cell Sci., vol. 122, no. 9, pp. 1285-1294, 2009.

[33] K. R. Feingold, "Thematic review series: Skin Lipids . The role of epidermal lipids in cutaneous permeability barrier homeostasis: Fig. 1.," J. Lipid Res., vol. 48, no. 12, pp. 2531-2546, 2007.

[34] M. Mildner et al., "Knockdown of filaggrin impairs diffusion barrier function and increases UV sensitivity in a human skin model," J. Invest. Dermatol., vol. 130, no. 9, pp. 2286-2294, 2010.

[35] A. Sandilands, C. Sutherland, A. D. Irvine, and W. H. I. McLean, "Filaggrin in the frontline: role in skin barrier function and disease.," J. Cell Sci., vol. 122, no. Pt 9, pp. 1285-94, May 2009.

[36] S. Jaitley and T. Saraswathi, "Pathophysiology of Langerhans cells.," J. Oral Maxillofac. Pathol., vol. 16, no. 2, pp. 239-44, 2012.

[37] C. Oba et al., "Dietary milk sphingomyelin prevents disruption of skin barrier function in hairless mice after UV-B irradiation," PLoS One, vol. 10, no. 8, pp. 110, 2015.

[38] M. Malumbres and M. Barbacid, "Cell cycle, CDKs and cancer: a changing pardigm," Nat. Rev. Cancer, vol. 9, 2009.

[39] X. Liu et al., "Acid Ceramidase Up-regulation in Prostate Cancer: Role in Tumor Development and Implications for Therapy," Expert Opin. Ther. Targets, vol. 13, no. 12, pp. 1449-1458, 2010.

[40] J. D'Orazio, S. Jarrett, A. Amaro-Ortiz, and T. Scott, "UV radiation and the skin," Int. J. Mol. Sci., vol. 14, no. 6, pp. 12222-12248, 2013.

[41] L. Pray, "DNA replication and causes of mutation," Nat. Educ., vol. 1, no. 1, p. 214, 2008.

[42] B. B. Vogelstein, "p53 : The Most Frequently Altered Gene in Human Cancers," Nat. Educ., vol. 3, no. 6, pp. 1-7, 2014.

[43] L. L. Mays Hoopes, “Aging and Cell Division,” Nature, vol. 3, no. 2010, pp. 9-12, 2010.

[44] B. Alberts, A. Johnson, and J. Lewis, Molecular Biology of the Cell: An Overview of the Cell Cycle, vol. 3169. 2002.

[45] L. A. Baker et al., "Probing the ultrafast energy dissipation mechanism of the sunscreen oxybenzone after UVA irradiation," J. Phys. Chem. Lett., vol. 6, no. 8, pp. 1363-1368, 2015.

[46] E. Fahy et al., "A comprehensive classification system for lipids," J. Lipid Res., vol. 46, no. 5, pp. 839-862, 2005.

[47] D. K. Breslow, "Sphingolipid homeostasis in the endoplasmic reticulum and beyond," Cold Spring Harb. Perspect. Biol., vol. 5, no. 4, pp. 1-16, 2013. 
[48] E. Fahy, M. Sud, D. Cotter, and S. Subramaniam, "LIPID MAPS online tools for lipid research," Nucleic Acids Research, 2007. .

[49] D. K. Breslow and J. S. Weissman, "Membranes in Balance: Mechanisms of Sphingolipid Homeostasis," Mol. Cell, vol. 40, no. 2, pp. 267-279, 2010.

[50] Y. A. Hannun, "The sphingomyelin cycle and the second messenger function of ceramide," J. Biol. Chem., vol. 269, no. 5, pp. 3125-3128, 1994.

[51] T. Y. Wang and J. R. Silvius, "Different sphingolipids show differential partitioning into sphingolipid/cholesterol-rich domains in lipid bilayers," Biophys. J., vol. 79, no. 3, pp. 1478-1489, 2000.

[52] B. M. Castro, M. Prieto, and L. C. Silva, "Ceramide: A simple sphingolipid with unique biophysical properties," Prog. Lipid Res., vol. 54, no. 1, pp. 53-67, 2014.

[53] T. Beckham, J. Cheng, S. T. Marrison, J. Norris, and X. Liu, "Interdiction of Sphingolipid Metabolism to Improve Standard Cancer Therapies," vol. 117, no. 1, pp. 1-36, 2013.

[54] R. Hage-Sleiman, M. O. Esmerian, H. Kobeissy, and G. Dbaibo, "P53 and Ceramide As Collaborators in the Stress Response," Int. J. Mol. Sci., vol. 14, no. 3, pp. 4982-5012, 2013.

[55] J. Liu, B. Beckham, and M. Foroozesh, "A review of ceramide analogs as potential anticancer agents," Future Med. Chem., vol. 5, no. 12, pp. 1405-1421, 2013.

[56] R. Jugé, J. Breugnot, C. Da Silva, S. Bordes, B. Closs, and A. Aouacheria, "Quantification and characterization of UVB-induced mitochondrial fragmentation in normal primary human keratinocytes," Sci. Rep., vol. 6, no. September, pp. 1-9, 2016.

[57] E. Sezgin and P. Schwille, "Fluorescence techniques to study lipid dynamics," Cold Spring Harb. Perspect. Biol., vol. 3, no. 11, pp. 1-32, 2011.

[58] M. Hao and F. R. Maxfield, "Characterization of rapid membrane internalization and recycling,” J. Biol. Chem., vol. 275, no. 20, pp. 15279-15286, 2000.

[59] J. W. Kok, T. Babia, K. Klappe, and D. Hoekstra, "Fluorescent, short-chain C6NBD-sphingomyelin, but not C6-NBD-glucosylceramide, is subject to extensive degradation in the plasma membrane: implications for signal transduction related to cell differentiation.," Biochem. J., vol. 309 ( Pt 3, pp. 905-12, 1995.

[60] M. Koval and R. E. Pagano, "Lipid recycling between the plasma membrane and intracellular compartments: Transport and metabolism of fluorescent sphingomyelin analogues in cultured fibroblasts," J. Cell Biol., vol. 108, no. 6, pp. 2169-2181, 1989.

[61] “Avanti Polar Lipids,” 2018. [Online]. Available: http://www.avantilipids.com/index.php?option=com_content\&view=article\&id=22 $04 \&$ Itemid $=191 \&$ catnumber $=850338$. 
[62] A. S. Klymchenko and R. Kreder, "Fluorescent probes for lipid rafts: From model membranes to living cells," Chem. Biol., vol. 21, no. 1, pp. 97-113, 2014.

[63] B. D. Grant and J. G. Donaldson, "Pathways and mechanisms of endocytotic recycling," Mol. Cell Biol., vol. 10, no. 9, pp. 597-604, 2009.

[64] A. Yamaji et al., "Lysenin, a novel sphingomyelin-specific binding protein," $J$. Biol. Chem., vol. 273, no. 9, pp. 5300-5306, 1998.

[65] A. Mound et al., "Non-senescent keratinocytes organize in plasma membrane submicrometric lipid domains enriched in sphingomyelin and involved in reepithelialization," Biochim. Biophys. Acta - Mol. Cell Biol. Lipids, vol. 1862, no. 9, pp. 958-971, 2017.

[66] R. Corriden et al., "Tamoxifen augments the innate immune function of neutrophils through modulatin of intracellular ceramide," 2016.

[67] F. J. O’Brien, "Biomaterials \& scaffolds for tissue engineering," Mater. Today, vol. 14, no. 3, pp. 88-95, 2011.

[68] K. Vig et al., "Advances in skin regeneration using tissue engineering," Int. J. Mol. Sci., vol. 18, no. 4, 2017.

[69] Y. Nashimoto, T. Hayashi, I. Kunita, A. Nakamasu, and R. Yokokawa, "Integrating perfusable vascular networks with a three-dimensional tissue in a microfluidic device," Integr. Biol., no. 9, pp. 506-518, 2017.

[70] C. F. Cho et al., "Blood-brain-barrier spheroids as an in vitro screening platform for brain-penetrating agents," Nat. Commun., vol. 8, pp. 1-14, 2017.

[71] E. Urich, C. Patsch, S. Aigner, M. Graf, R. Iacone, and P. O. Freskgård, "Multicellular self-assembled spheroidal model of the blood brain barrier," Sci. Rep., vol. 3, 2013.

[72] H. P. H. Naber, E. Wiercinska, P. ten Dijke, and T. van Laar, "Spheroid Assay to Measure TGF- $\beta$-induced Invasion,” J. Vis. Exp., no. 57, pp. 1-7, 2011.

[73] S. M. Ehsan et al., "A three-dimensional in vitro model of tumor cell intravasation,” Integr. Biol., vol. 6, no. 6, pp. 603-610, 2014.

[74] C. Wong, E. Vosburgh, A. J. Levine, L. Cong, and E. Y. Xu, "Human Neuroendocrine Tumor Cell Lines as a Three-Dimensional Model for the Study of Human Neuroendocrine Tumor Therapy," J. Vis. Exp., no. 66, pp. 4-10, 2012.

[75] X. Gong et al., "Generation of multicellular tumor spheroids with microwell-based agarose scaffolds for drug testing," PLoS One, vol. 10, no. 6, pp. 1-18, 2015.

[76] D. P. Ivanov and A. M. Grabowska, "Spheroid arrays for high-throughput singlecell analysis of spatial patterns and biomarker expression in 3D," Sci. Rep., vol. 7, no. December 2016, pp. 1-12, 2017.

[77] M. E. Katt, A. L. Placone, A. D. Wong, Z. S. Xu, and P. C. Searson, "In Vitro Tumor Models: Advantages, Disadvantages, Variables, and Selecting the Right 
Platform," Front. Bioeng. Biotechnol., vol. 4, no. February, 2016.

[78] B. Leung, S. C. Lesher-Perez, T. Matsuoka, C. Moraes, and S. Takayama, "Media additives to promote spheroid circularity and compactness in hanging drop platform," Biomater. Sci., vol. 3, pp. 336-344, 2015.

[79] J. Klicks, E. von Molitor, T. Ertongur-Fauth, R. Rudolf, and M. Hafner, "In vitro skin three-dimensional models and their applications," J. Cell. Biotechnol., vol. 3, no. 1, pp. 21-39, 2017.

[80] Syntivia, "Mixed Spheroids of Melanocytes and Keratinocytes," US 2017/0248582, 2017.

[81] C. S. Hughes, L. Radan, D. Betts, L. M. Postovit, and G. A. Lajoie, "Proteomic analysis of extracellular matrices used in stem cell culture," Proteomics, vol. 11, no. 20, pp. 3983-3991, 2011.

[82] ThermoFisher Scientific, "NBD- and BODIPY Dye - Labeled Sphingolipids," 2003. .

[83] S. C. D. Van IJzendoorn, M. M. P. Zegers, J. W. Kok, and D. Hoekstra, "Segregation of glucosylceramide and sphingomyelin occurs in the apical to basolateral transcytotic route in HepG2 cells," J. Cell Biol., vol. 137, no. 2, pp. 347-357, 1997.

[84] T. Kobayashi, A. Makino, and K. Ishii, "Vital Staining of Cells with Fluorescent Lipids," in Cell Biology, 2006, pp. 139-145.

[85] R. Wanner, M. Peiser, and B. Wittig, "Keratinocytes rapidly readjust ceramidesphingomyelin homeostasis and contain a phosphatidylcholine-sphingomyelin transacylase," J. Invest. Dermatol., vol. 122, no. 3, pp. 773-782, 2004.

[86] J. A. Pike, I. B. Styles, J. Z. Rappoport, and J. K. Heath, "Quantifying receptor trafficking and colocalization with confocal microscopy," vol. 115, pp. 42-54, 2017. 


\section{APPENDICES}

\section{A. 2D Fluorescence Distribution Codes}

The following ImageJ and MATLAB codes were written by Tyler Fraser and Stephanie Switalski.

\section{FSM Doughnut ROI}

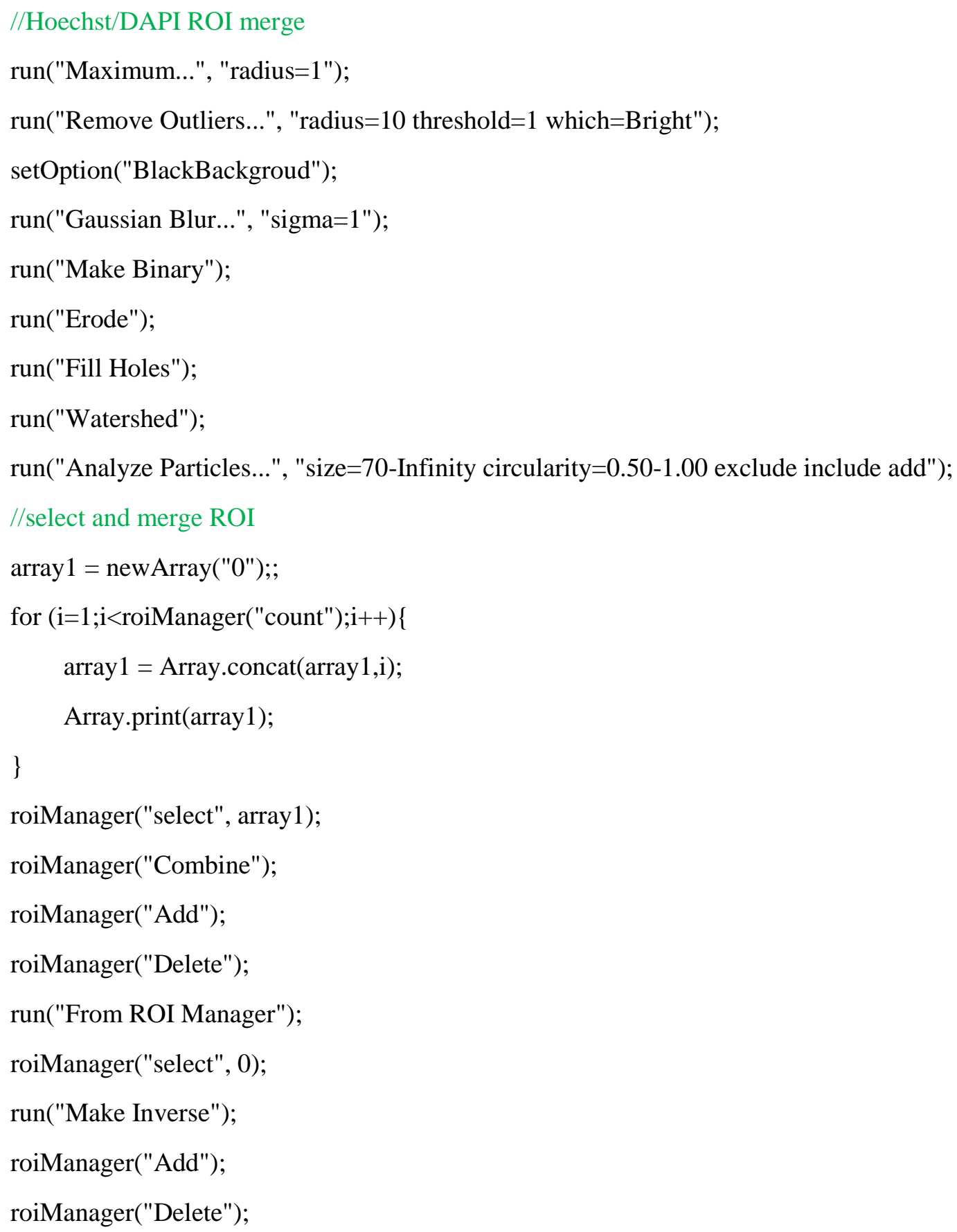




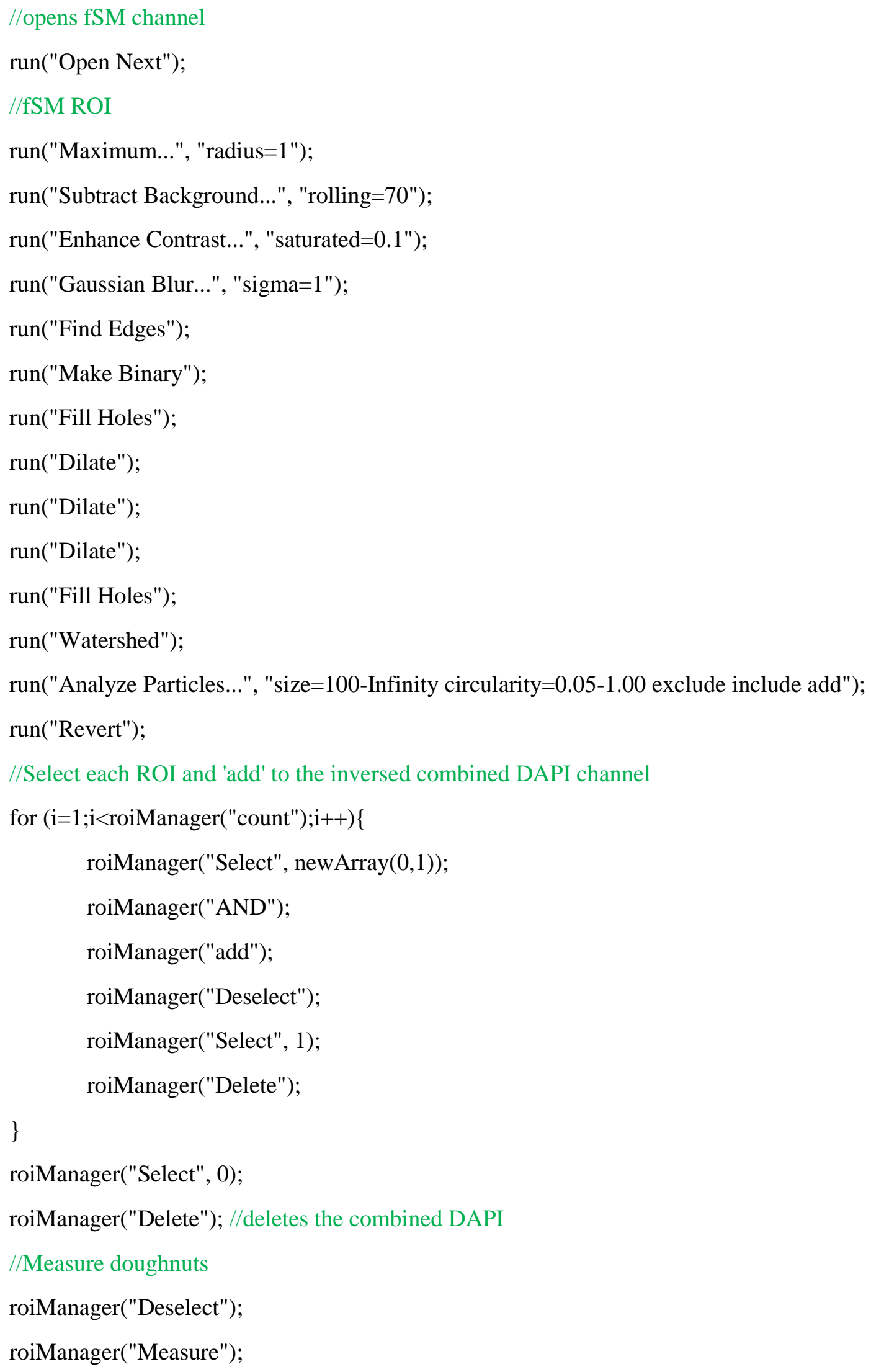




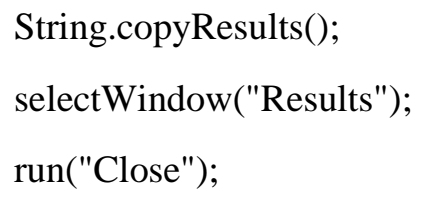




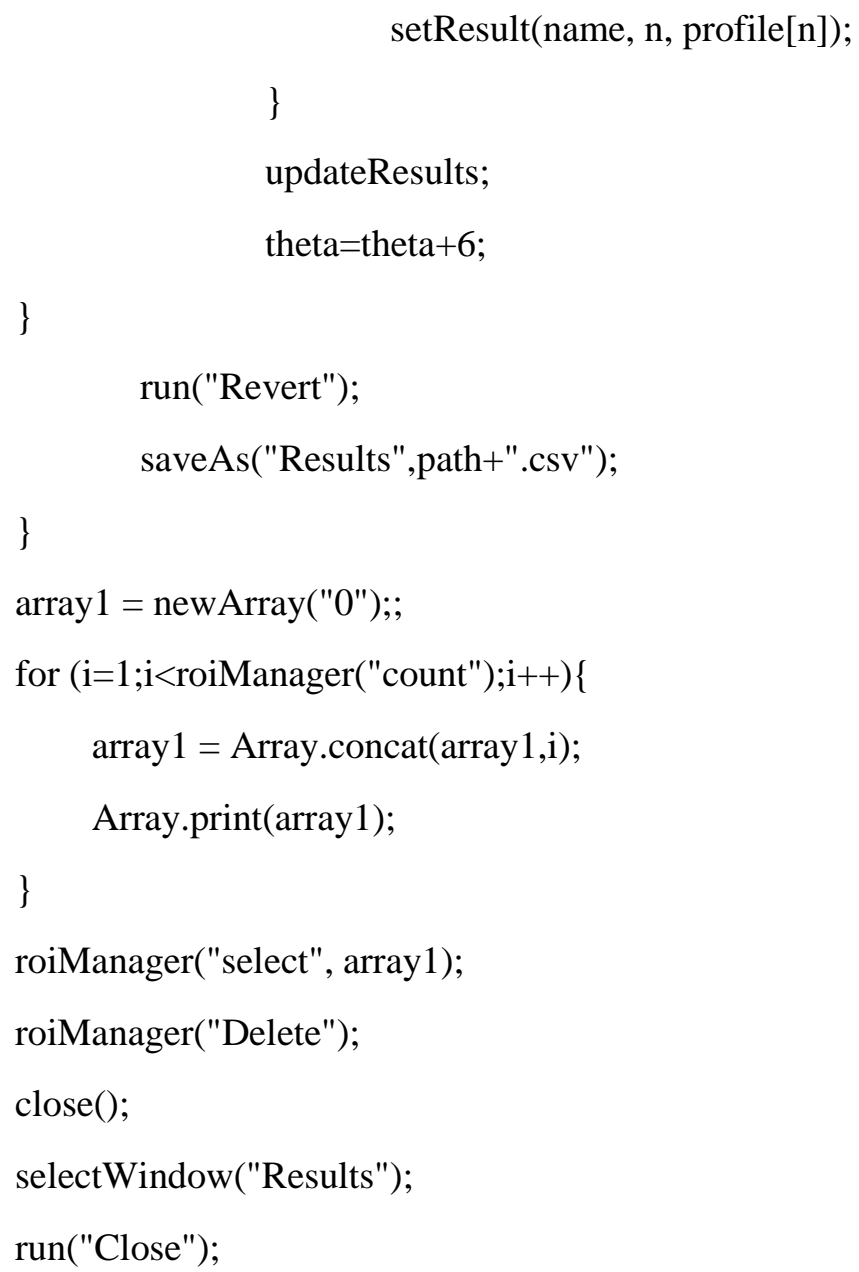




\section{MATLAB Code}

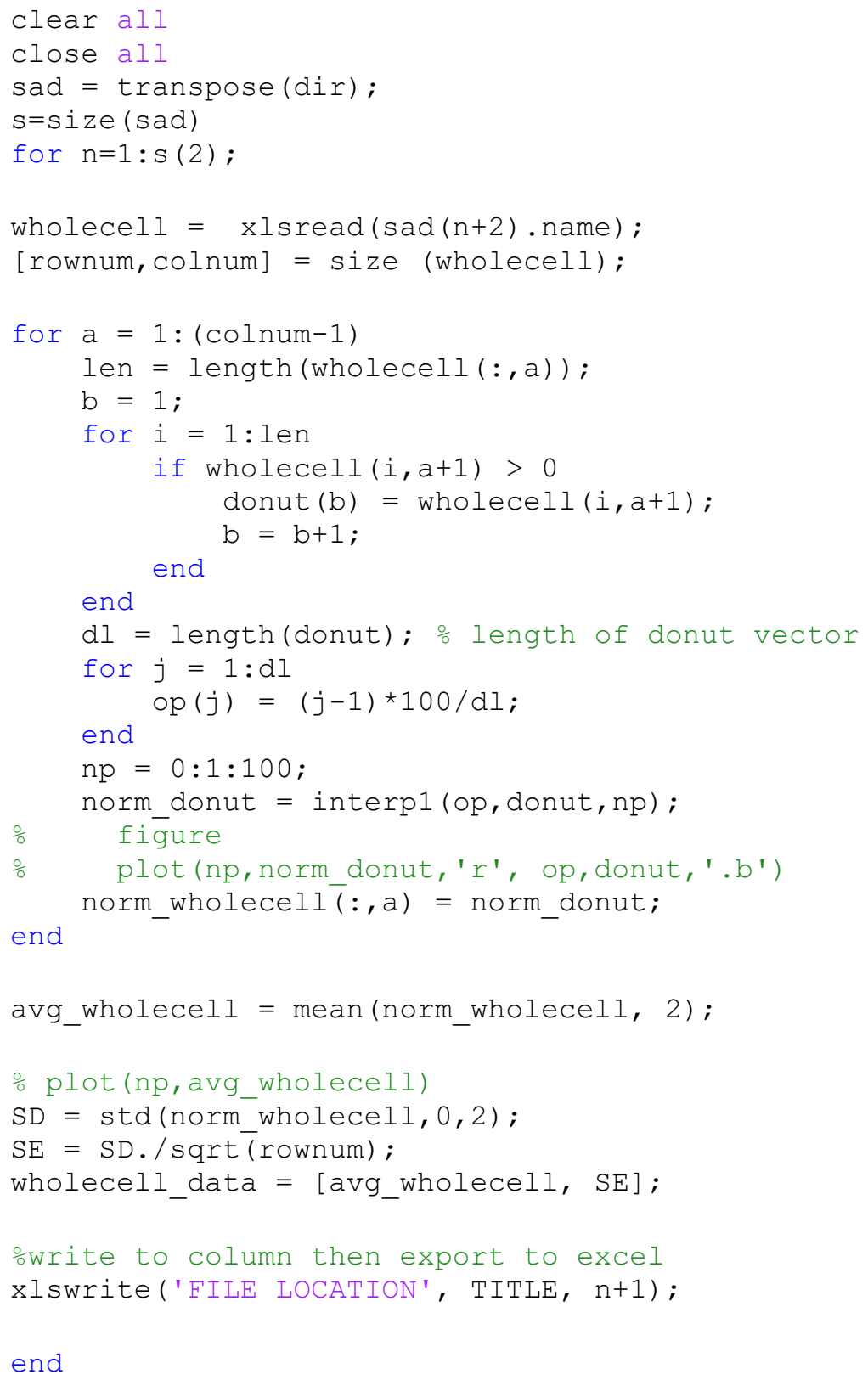

end 


\section{B. Normality Assumptions (from JMP)}

Normal quantile plots and one-way Analysis of Residuals are included for each experiment. The normal quantile plots appear reasonably linear so there is no substantial evidence of nonnormality in the residuals. All data falls within the bounded red lines. Furthermore, slight differences in variation among the treatment groups are apparent, but the differences are not extreme. There is no substantial evidence of unequal variance. Independence is met by assuming each well is an experimental unit.

\section{D FSM Fluorescence Distribution Trafficking}

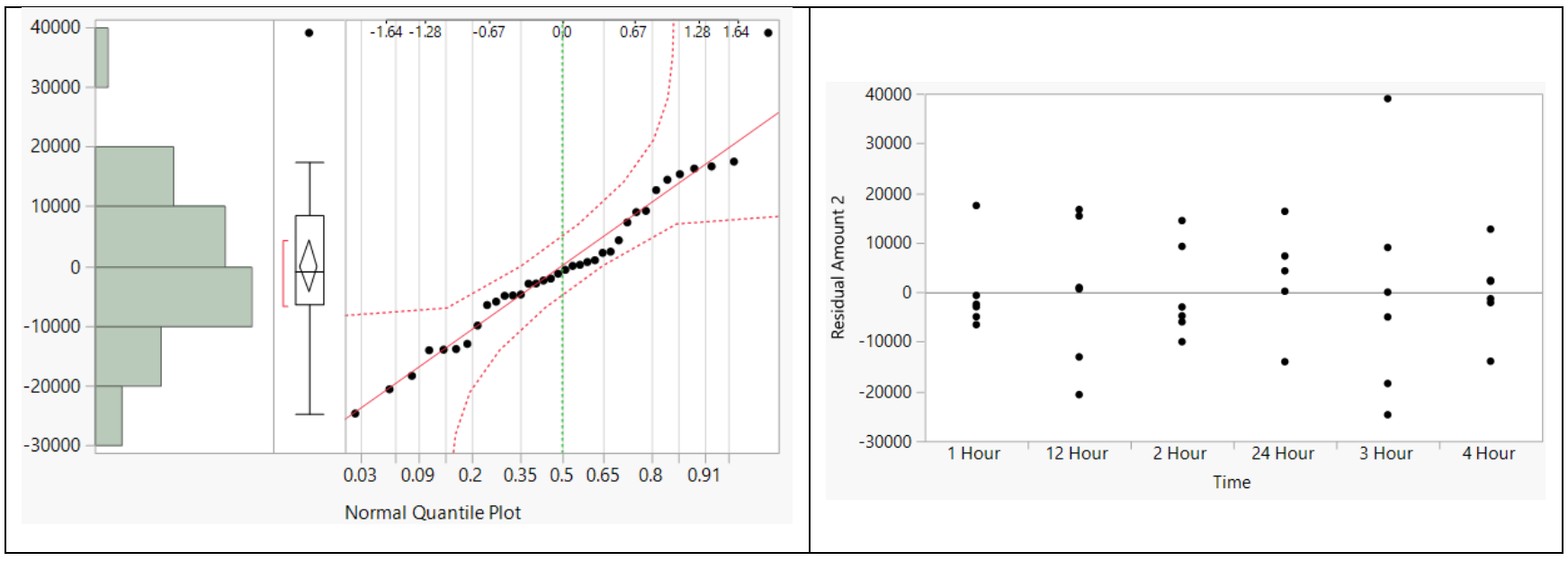

\section{UV FSM Fluorescence Distribution Trafficking}

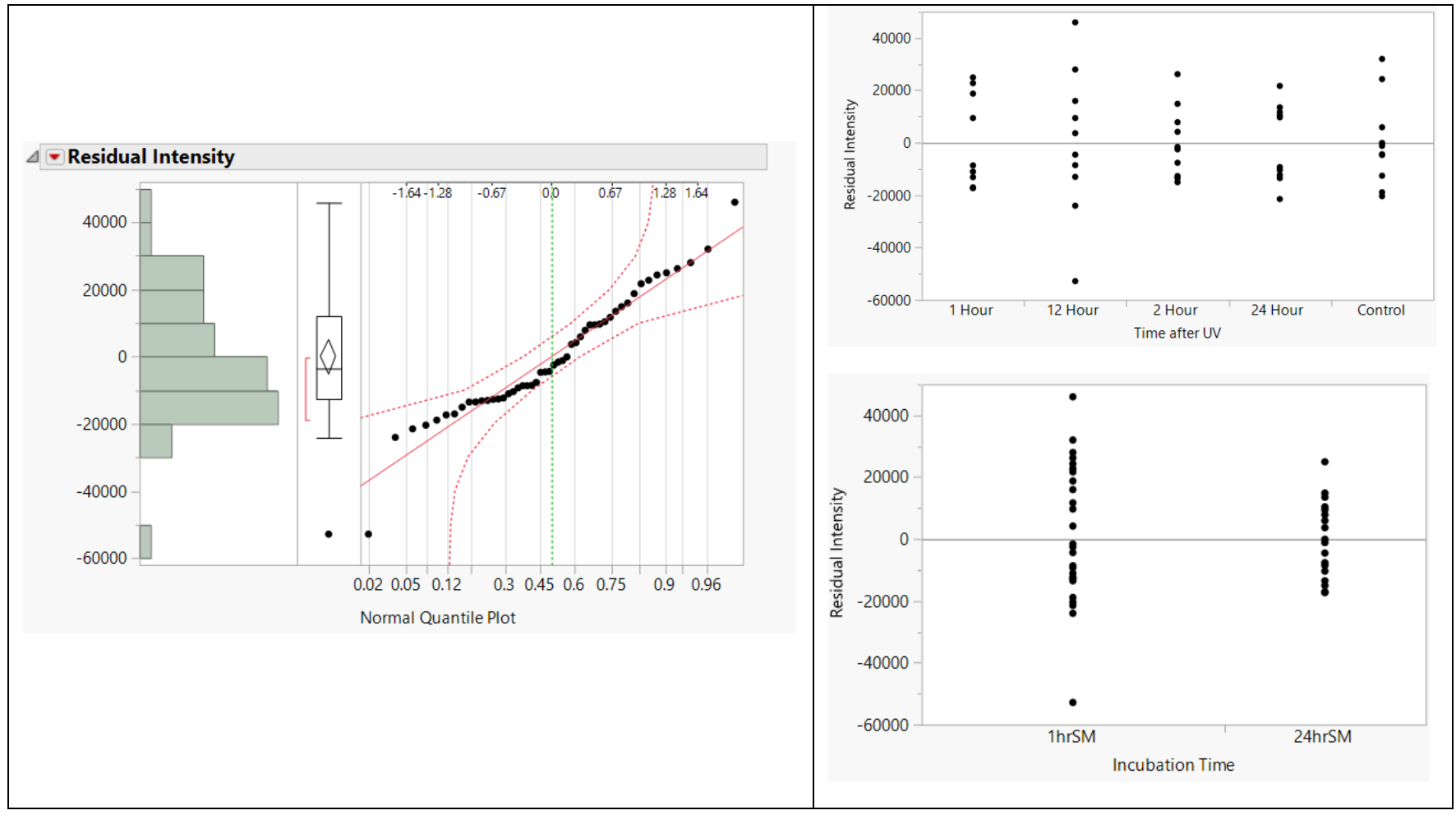


BSM p21 Ratios

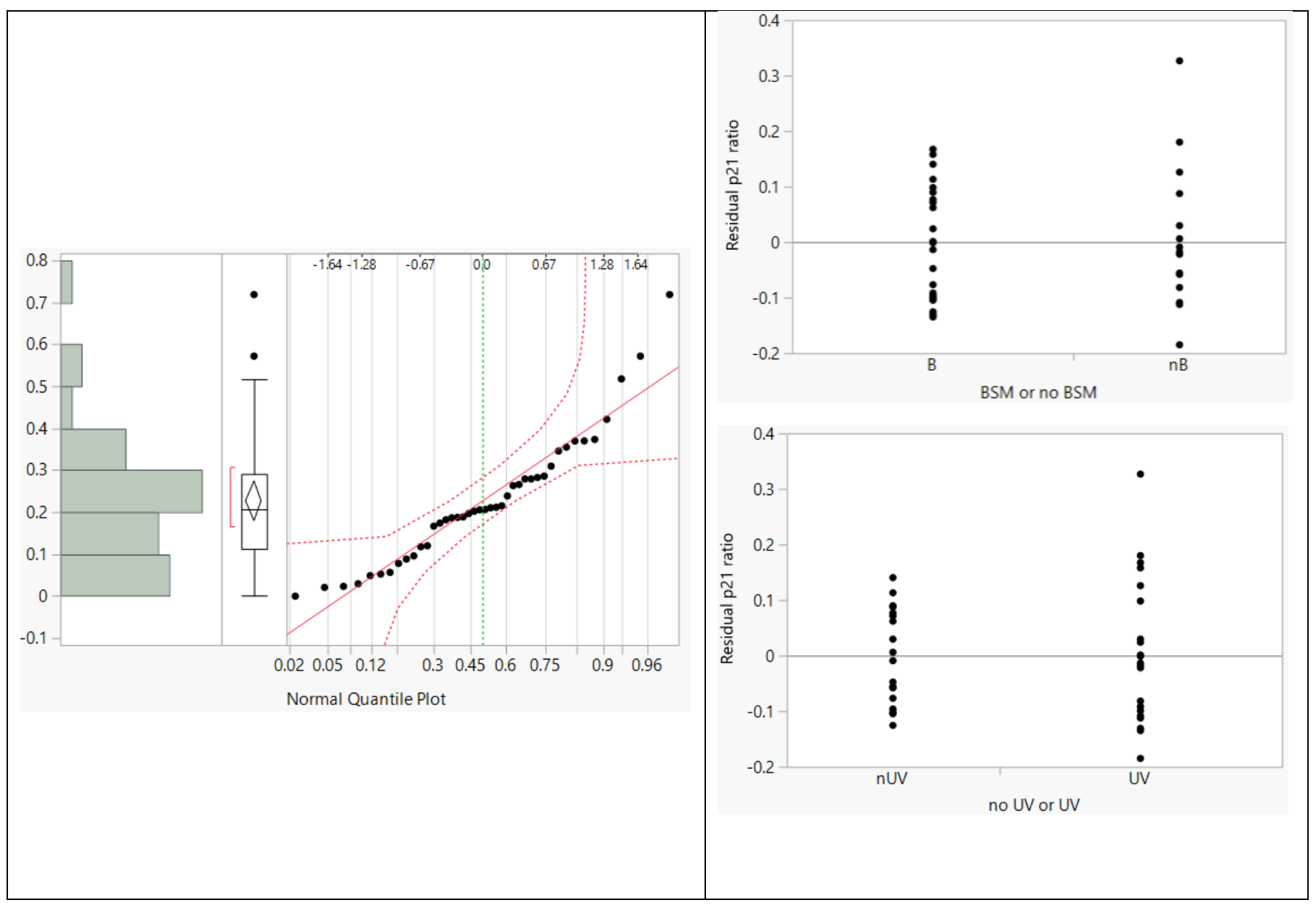


FSM p21 Ratios

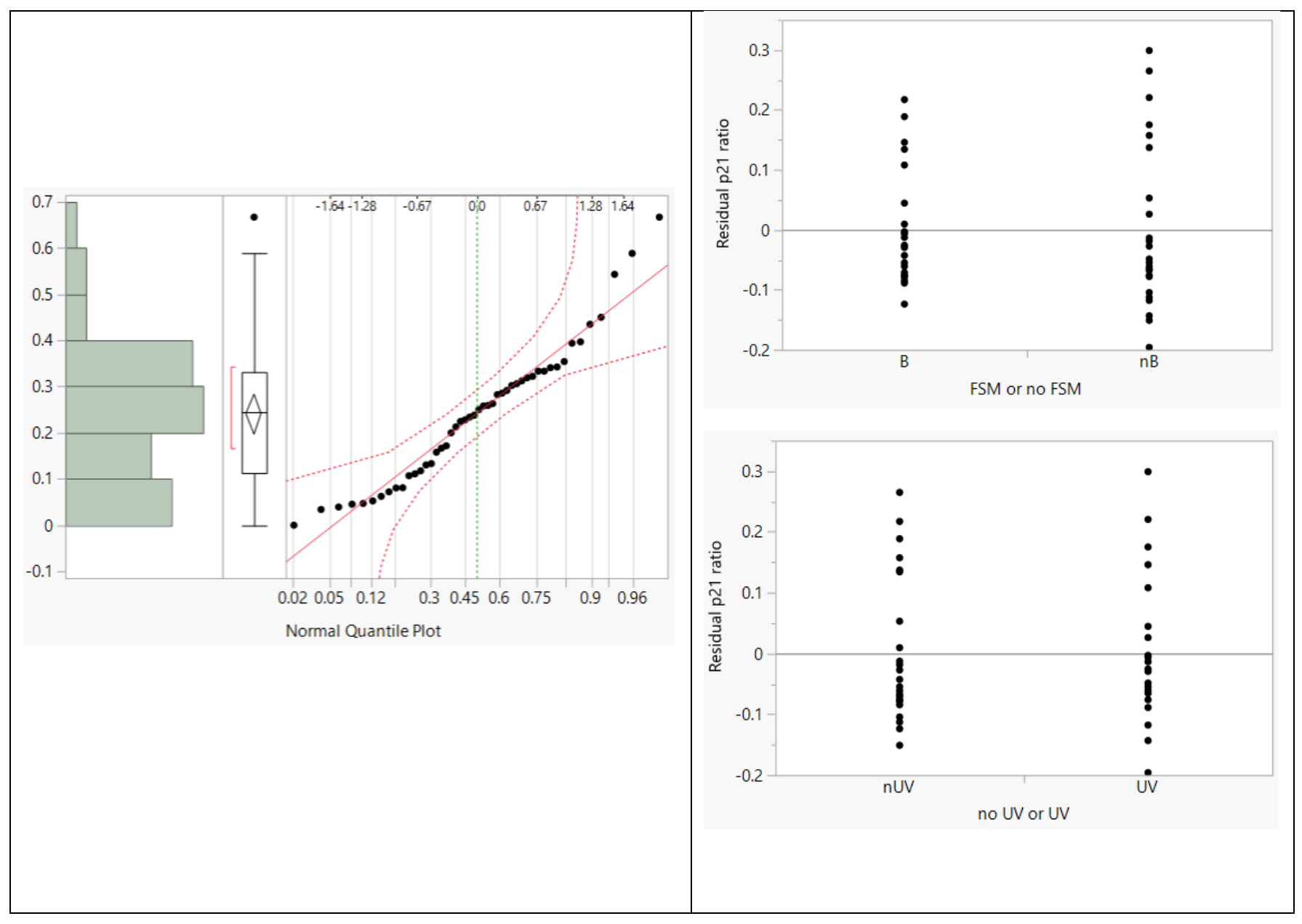


FSM, Lysenin, and Ceramide 2D Fluorescence Distribution Trafficking

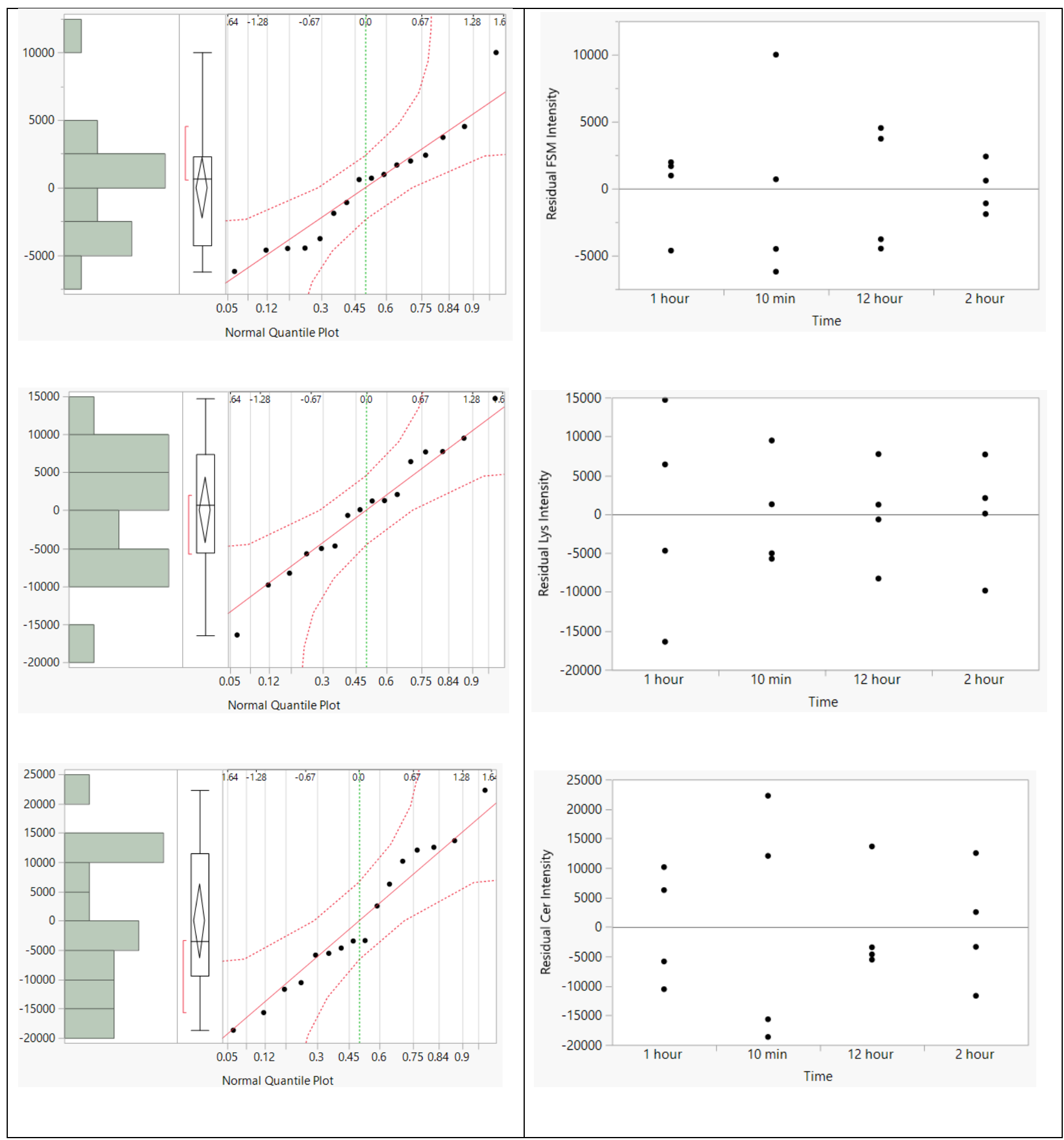

